\title{
Gaming for safer sex
}

Citation for published version (APA):

Brüll, P. (2019). Gaming for safer sex: improving explicit and implicit cognitions toward safer sex precautions in young people. [Doctoral Thesis, Maastricht University]. ProefschriftMaken Maastricht. https://doi.org/10.26481/dis.20190206pb

Document status and date:

Published: 01/01/2019

DOI:

10.26481/dis.20190206pb

Document Version:

Publisher's PDF, also known as Version of record

\section{Please check the document version of this publication:}

- A submitted manuscript is the version of the article upon submission and before peer-review. There can be important differences between the submitted version and the official published version of record.

People interested in the research are advised to contact the author for the final version of the publication, or visit the DOI to the publisher's website.

- The final author version and the galley proof are versions of the publication after peer review.

- The final published version features the final layout of the paper including the volume, issue and page numbers.

Link to publication

\footnotetext{
General rights rights.

- You may freely distribute the URL identifying the publication in the public portal. please follow below link for the End User Agreement:

www.umlib.nl/taverne-license

Take down policy

If you believe that this document breaches copyright please contact us at:

repository@maastrichtuniversity.nl

providing details and we will investigate your claim.
}

Copyright and moral rights for the publications made accessible in the public portal are retained by the authors and/or other copyright owners and it is a condition of accessing publications that users recognise and abide by the legal requirements associated with these

- Users may download and print one copy of any publication from the public portal for the purpose of private study or research.

- You may not further distribute the material or use it for any profit-making activity or commercial gain

If the publication is distributed under the terms of Article $25 \mathrm{fa}$ of the Dutch Copyright Act, indicated by the "Taverne" license above, 


\section{Gaming for Safer Sex:}

Improving Explicit and Implicit Cognitions toward

Safer-Sex Precautions in Young People

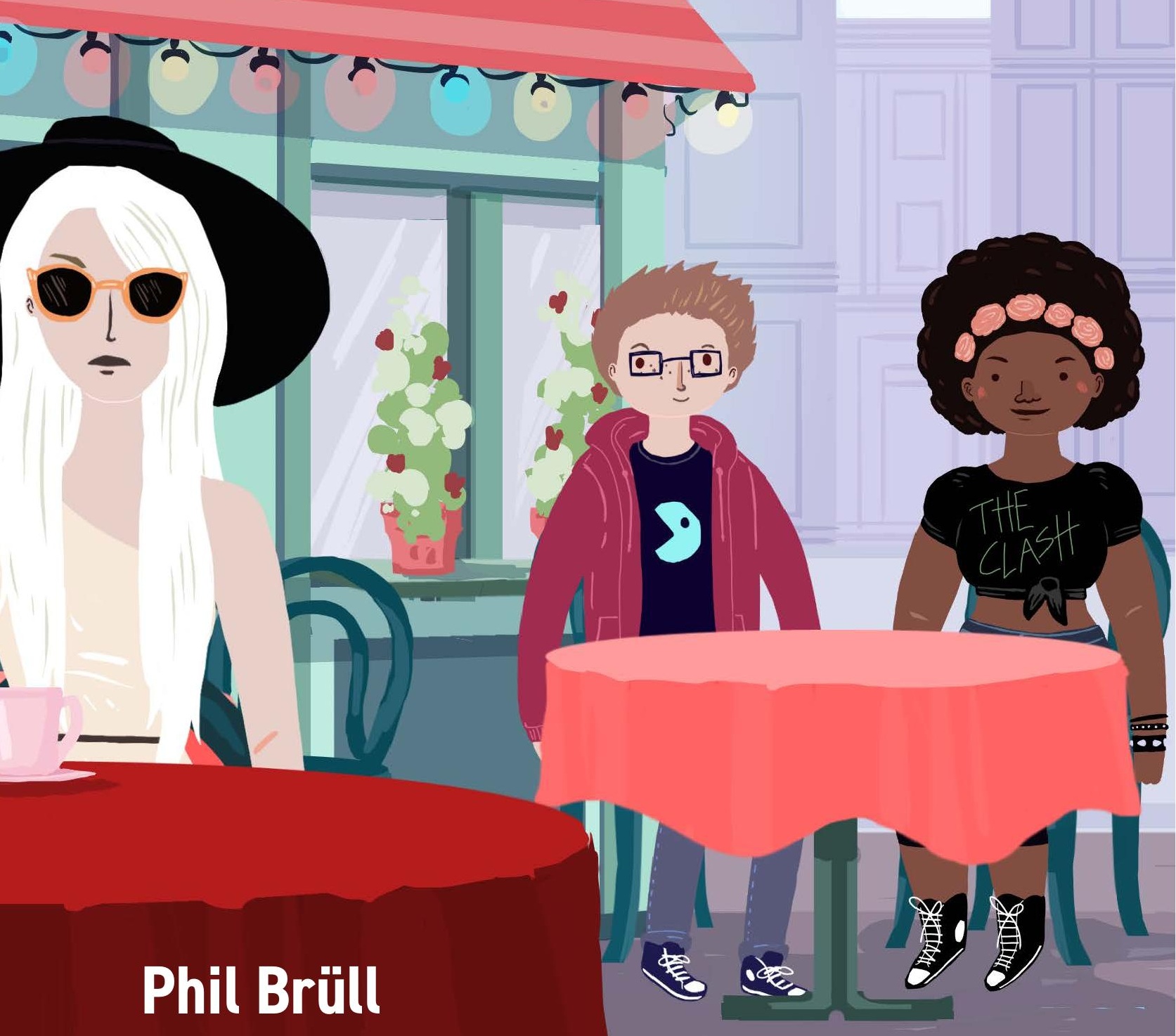





\section{Gaming for Safer Sex:}

\section{Improving Explicit and Implicit Cognitions toward Safer-Sex Precautions in Young People}


(C) copyright Phil Brüll, Maastricht 2019

Printing: ProefschriftMaken || www.proefschriftmaken.nl

ISBN 978-94-6380-230-7

All rights reserved. No part of thwis publication may be reproduced, stored in a retrieval system or transmitted, in any form or by any means, electronic, mechanical, photocopying, recording or otherwise, without prior permission of the author or the copyright-owning journals for previous published chapters. 


\title{
Gaming for Safer Sex:
}

\section{Improving Explicit and Implicit Cognitions toward Safer-Sex Precautions in Young People}

\author{
Dissertation \\ to obtain the degree of Doctor at Maastricht University, \\ on the authority of the Rector Magnificus Prof. dr. Rianne M. Letschert \\ in accordance with the decision of the Board of Deans, \\ to be defended in public on \\ Wednesday, $6^{\text {th }}$ February 2019, at 12:00 hours
}

by

Phil Brüll 


\section{Promotors}

Prof. Dr. R.A.C. Ruiter, Maastricht University

Prof. Dr. R.W. Wiers, University of Amsterdam

Prof. Dr. G. Kok, Maastricht University

\section{Assessment Committee}

Prof. Dr. H. Hospers, Maastricht University

Prof. Dr. C. Hoebe, Maastricht University

Prof. Dr. A. Bos, Open Universiteit Nederland

Dr. K. Jonas, Maastricht University

Dr. A. DeSmet, Universiteit Gent 


\section{Table of Contents}

General Introduction $\quad 11$

Young people and STIs 13

Risk factors in young people 13

Young people and determinants of behavior $\quad 19$

Explicit determinants of behavior $\quad 20$

Implicit determinants of behavior 20

Young people and safer-sex education programs $\quad 21$

Abstinence-only and comprehensive sex education $\quad 21$

Tailoring of sex education programs $\quad 22$

Young people and novel prevention strategies 23

Serious or educational games $\quad 23$

An outlook on the future of serious games for young people 24

Research objectives and outline of the dissertation 25

Chapter 1 Identifying Psychosocial Variables that Predict Safer-sex Intentions in $\begin{array}{ll}\text { Adolescents and Young Adults } & 29\end{array}$

$\begin{array}{ll}\text { Abstract } & 30\end{array}$

Introduction 31

Material and Methods 33

Participants and procedure $\quad 33$

Measures $\quad 34$

Distal variables 34

Knowledge $\quad 35$

RAA variables $\quad 35$

$\begin{array}{ll}\text { Statistical analysis } & 37\end{array}$

Results $\quad 37$

$\begin{array}{ll}\text { Demographics } & 37\end{array}$

Factorial analysis 38

Univariate associations $\quad 38$

Regression analysis $\quad 42$

Moderation analysis $\quad 42$

Discussion 43

Intention to ask a new sex partner about his/her health status $\quad 44$

Intention to perform less risky sex acts with a new partner 44

Intention to use condoms with a new partner $\quad 45$ 
Chapter 2 "Don't take my fun away!": Expectations of reduced hedonic experience and embarrassment impede implementation of additional safer-sex behavior

Abstract

Introduction

Materials and methods

Participants and procedure

Measures

Data processing and analysis

Results

Risky sex acts

Asking about sexual health status 55

Condom use 56

Discussion 61

Conclusion 65

Chapter 3 Gaming For Safer-Sex: Young German and Turkish People Report no Specific Culture-Related Preferences Towards Educational Games Promoting Safer Sex

Abstract

Introduction

Materials and Methods

Participants and procedure 71

Study setting 73

Data processing and analysis 74

Results 75

Realistic real-world design 75

Single-player or multi-player game 75

Avatars 76

Approach to sex 76

Health-related contexts 77

Intoxication 78

Imparting knowledge 78

Discussion 78

Conclusion 82 
Chapter 4 Different Levels of Sexual Arousal - Friends or Foes in Sexual

Abstract

Introduction

Explicit and implicit determinants of behavior

Methods

Participants

Procedure

Measures

Films to induce sexual arousal

Measurement of sexual arousal

Data analysis

Results

Induced sexual arousal 95

SC-IAT 95

Self-report questionnaires 96

Working memory

RAA model

Discussion

Conclusion

Chapter 5 ERPs Reveal Disengagement Processes During Condom Use Embarrassment in Intention-behavior Inconsistent Young Adults

Abstract

Introduction

Methods

Participants

107

Procedure

Measures

Analyses

Results

Error Analysis

Reaction Times

ERP Analyses

Self-Report Measures

Discussion

Conclusion 
Chapter 6 Heroes of the Night - Promoting Three Safer-Sex Behaviors by Targeting Explicit and Implicit Behavioral Determinants in a Serious Game

Abstract

Introduction

Explicit and implicit determinants of behavior

Working memory

Selective response inhibition

Evaluative conditioning

Methods

Participants

Procedure

Measures

Data analysis

Results

Active evaluative conditioning, active behavior inhibition, active working memory training

Active evaluative conditioning, active behavioral inhibition, mock working memory training

Active evaluative conditioning, mock behavioral inhibition, mock working memory training

Active behavioral inhibition, mock evaluative conditioning, mock working memory training

Mock behavioral inhibition, mock evaluative conditioning, mock working memory training

Active evaluative conditioning, active selective inhibition training, and active working memory training contrasted to mock conditions

Discussion

Limitations

Conclusion

Chapter 7 General Discussion

Determinants of behavior

Gamification of behavior-change interventions

The effectiveness of the game

Limitations

Implications for future research

Suggestions for future research

Conclusion

References

Appendix Reasoned Action Approach items adapted from Fishbein and Ajzen, 2010197 
Samenvatting

Valorization

209 

General Introduction 

In June 2016, the United Nations General Assembly High-Level Meeting on AIDS (Acquired Immune Deficiency Syndrome) adopted the United Nations Political Declaration on HIV (Human Immunodeficiency Virus) that aims to end the AIDS epidemic by 2030 (UNAIDS, 2016). However, the number of people living with HIV remains high (Globerman et al., 2017). In 2016, worldwide 36.7 million people were living with HIV (as compared to 35 million in 2013 and 33.3 million in 2010) and 1.8 million (as compared to 2.1 million in 2013 and 2.6 million in 2010) were newly infected (UNAIDS, 2011, 2014, 2017). In 2016, most people infected with HIV were living in eastern and southern Africa (19.4 million) and in western and central Africa (6.1 million), whereas 5.1 million were living in Asia and the Pacific, 2.1 million in western and central Europe and North America, 1.8 million in Latin America, 1.6 million in eastern Europe and central Asia, 310000 in the Caribbean, and 230000 in the Middle East and North Africa (UNAIDS, 2017).

\section{Young people and STIS}

Besides the key populations at risk to get infected with HIV, namely sex workers, men who have sex with men, people who inject drugs, transgender people, and prisoners (UNAIDS, 2018), young people aged between 15 and 24 years are also at risk (Piot et al., 2015; Shannon \& Klausner, 2018) but rarely prioritized in national HIV prevention programs (Pettifor, Stoner, Pike, \& Bekker, 2018). In 2016, worldwide, there were 2.1 million young people aged between 10 and 19 years living with HIV (UNICEF, 2017) and 607000 young people aged between 15 and 24 years became newly infected with HIV (UNAIDS, 2017). However, young people are not only at risk to get infected with HIV, but they are also at high risk to acquire other sexually transmitted infections (STIS) (Centers for Disease Control and Prevention, 2017). Every year, worldwide, a quarter of a billion young people aged 15-24 years acquire a curable STI for the first time. This accounts for nearly half of all new curable STIs (World Health Organization, 2013) and is of particular importance, as sexually transmitted diseases (STDs) facilitate the HIV transmission through direct, biological mechanisms (Fleming \& Wasserheit, 1999). Furthermore, once infected with HIV, the risk of HIV transmission remains substantial, even during asymptotic stages (Hollingsworth, Anderson, \& Fraser, 2008; Kim \& Koopman, 2012).

\section{Risk factors in young people}

Young people nearly comprise a quarter of the world's population, and nearly $90 \%$ live in low- or middle-income countries (United Nations, 2015). Young people, defined as individuals aged between 15 and 24 years (Sawyer et al., 2012), just emerged out of the transition from puberty to independence and self-sufficiency (World Health Organization, 2001) after substantial biological, psychological and behavioral developments (Sharma et 
al., 2013). As gaining sexual maturity is part of these developments it comes to no surprise that safer-sex behavior is complex. In fact, it includes sub-behaviors such as evolved patterns of sexual partnering and promiscuity in increasingly heterogeneous sexual networks in emerging cohorts (Nahmias \& Nahmias, 2011). The following is a non-hierarchical and non-exhaustive list of well-documented risk factors related to young people's sexuality.

\section{Socioeconomic status (SES) and cultural factors}

The prevalence rate of STIs are higher among young people from racial/ethnic minorities, and socioeconomically disadvantaged families (Centers for Disease Control and Prevention, 2017), mostly due to exposure-dependent mechanisms (e.g., adversities associated with family or community that may promote risky sexual behaviors) and individual-level characteristics, such as individual life histories or emotional adjustment difficulties (Wickrama, Merten, \& Wickrama, 2012). In Western Europe, people from racial or ethnic minorities tend to engage more often in unprotected sexual intercourse than people belonging to the respective ethnic majority (Dias, Marques, Gama, \& Martins, 2014; Uccella et al., 2017). Further, young people living in low-income settings tend to have an earlier initiation of heterosexual intercourse compared to people living in higher-income settings (Cubbin, Brindis, Jain, Santelli, \& Braveman, 2010). In low-income settings, girls are at an elevated risk for HIV, STIs, and unintended pregnancies (Morrison-Beedy et al., 2012), also due to a high number of sexual partners (Van Ryzin, Johnson, Leve, \& Kim, 2011) and especially if their SES is inferior to their partner's SES (Muchomba, Chan, \& ElBassel, 2015; Sales et al., 2014).

\section{Peer and parents}

The relationship between young people and their parents and peers is dynamic, going through different phases of conformity and opposition (Berndt, 1979). It has been shown that lower parental monitoring predicts a greater number of sexual partners at age 16 , whereas higher parental monitoring predicts a growth in number of sexual partners at the ages following 16. In fact, a better predictor than parental monitoring of the number of sexual partners seems to be early adolescent developmental antecedents (Lansford et al., 2010), as a younger age of first sexual intercourse is associated with having more sexual partners (Seidman, Mosher, \& Aral, 1994). However, parental connectedness (or bonding), especially if paired with sexuality-specific parent-adolescent communication (as opposed to parental overcontrol), has shown to be a protective factor of young people's sexual and reproductive health (Markham et al., 2010). Even if parent's communication with their children about safer-sex behavior can be improved (Hyde et al., 2013), it has been shown that parenting style and parent-child communication can have a positive impact on young people's safer-sex behavior (e.g., Huebner \& Howell, 2003; LaSala, 2007). Peers, on the contrary, have been associated with increased risk-taking by young people due to a resulting enhancement of activity in the brain's reward circuitry (e.g., 
Albert, Chein, \& Steinberg, 2013; Chein, Albert, O'Brien, Uckert, \& Steinberg, 2011; Smith, Chein, \& Steinberg, 2014). Especially peer deviance has been identified as a salient predictor of risky sexual behaviors in specific sub-populations, such as African American adolescents (Young \& Vazsonyi, 2011). However, young people tend to make riskier decisions when they are merely observed by peers - and also tend to go against safe advice pronounced by peers - compared to being in a private setting (Haddad, Harrison, Norman, \& Lau, 2014). Peers do have a normative influence on young people, also regarding sexual behavior (Coley, Lombardi, Lynch, Mahalik, \& Sims, 2013), and have to be taken into account when analyzing young people's sexual behavior.

\section{Religiosity}

A negative relationship between young people's sexual behavior and religion has been established in past research (e.g., Fletcher \& Kumar, 2014; Rostosky, Wilcox, Wright, \& Randall, 2004; Sinha, Cnaan, \& Gelles, 2007). Young people who are affiliated with religious peers who discourage or disapprove of risky sexual behavior show less of such risky behavior. However, the effect of religiosity seems to be intertwined with a normative influence of peers, where safer-sex behavior or abstinence could then become a peer group norm (Landor, Simons, Simons, Brody, \& Gibbons, 2011; Langlais \& Schwanz, 2018). Closeness to parents paired with participation in religious activities was identified as a strongly inhibiting factor of risky sexual behavior in young people (Longest \& Uecker, 2018). However, the effects of family religious socialization have shown to be unstable (Grossman, Tracy, \& Noonan, 2013).

\section{Substance use}

Illicit drug or alcohol use by young people has consistently been associated with risky sex behavior (Biglan et al., 1990; Hingson, Strunin, Berlin, \& Heeren, 1990; Lowry et al., 1994). However, outcomes depend on the kind of drug. Cigarette smokers, who did not escalate smoking, are more likely to implement consistent condom use compared to nonsmokers, whereas alcohol abuse is mostly associated with unsafe sex behavior (Guo et al., 2002). Especially binge drinking is associated with early initiation of sexual intercourse and more sexual partners (Holway, Tillman, \& Brewster, 2017). Interestingly, it has been shown that young people who frequently use marijuana, but not alcohol, engaged in less risky sex as compared to young people who either frequently use alcohol alone or both substances (Gillman, Yeater, Feldstein Ewing, Kong, \& Bryan, 2018). However, alcohol has been identified as an independent risk factor to engage in unprotected sex, and the role of alcohol consumption in the sexual health of young people cannot be underestimated (for a review, see Rehm, Shield, Joharchi, \& Shuper, 2012). 


\section{Sexual preferences}

Besides injection drug use, STIs, including HIV, are mainly spread by having unprotected anal or vaginal intercourse. The highest per-act probability of acquiring HIV is linked to receptive anal intercourse (138 per 10000 exposures), followed by insertive anal intercourse (11 per 10000 exposures), receptive penile-vaginal intercourse (8 per 10000 exposures), insertive penile-vaginal intercourse (4 per 10000 exposures), and receptive or insertive oral intercourse with a very low risk of infection (P. Patel et al., 2014a). Successful protection strategies against STI and HIV infections include the use of barrier protections, such as condoms or dental dams, during sexual intercourse, getting tested and knowing about a sex partner's STI and HIV test status, limiting the number of sexual partners, and the use of pre-exposure prophylaxis (PrEP) for people who are at high risk of becoming infected with HIV or post-exposure prophylaxis (PEP) for people who engaged in a single high-risk event (Centers for Disease Control and Prevention, 2018). However, some factors facilitating young people's risky sexual behavior, such as substance use (e.g., Lowry et al., 1994), are often correlated with environments that promote sexual sensation seeking (Gullette \& Lyons, 2005) or invite to have sexual intercourse with several partners, such as group sex parties (Buttram, Pagano, \& Kurtz, 2018).

\section{Sexting}

In young people, pornography use has been associated with more permissive sexual attitudes, a higher occurrence of sexual intercourse and casual sex behavior, as well as more sexual aggression (Peter \& Valkenburg, 2016). The rapid development of mobile communication devices that enable users to send and receive high-quality pictures and video sequences containing sexual content lead to highly interactive forms of modern communication (O'Sullivan, 2015). Sexting is defined as the private exchange of self-produced sexual images or video sequences via cell or smartphone or the internet (Döring, 2014). Especially adolescents below the age of 16 years have been associated with negative consequences due to sexting, such as increased sensation seeking, risky online and offline sexual behavior (Frankel, Bass, Patterson, Dai, \& Brown, 2018; Livingstone \& Görzig, 2014), and first-time sex with someone after sexting (FTSAS) which has been shown to be associated with the experience of unwanted sex and an increased risk for STIs (Boekeloo, Boyle, Quinton, \& Rashaw, 2018). However, the relation between sexting behavior and risky sexual behavior is mostly weak (for a review, see Kosenko, Luurs, \& Binder, 2017) and not constant across time (Ševčíková, Blinka, \& Daneback, 20018 )

\section{Type of sexual relationship}

Young people's sexual relationships tend to be brief (Henderson et al., 2002) and generally, safe sexual intercourse, especially condom use, is implemented inconsistently at best by the majority of sexually active young adults (Finer \& Philbin, 2013; Tschann, Flores, De 
Groat, Deardorff, \& Wibbelsman, 2010). Further, decisions about condom use are influenced by the nature accorded to a relationship, (i.e., sex only, boy-girlfriend, or a degree of affection mimicking marriage) where young people tend to use condoms less often if they consider their sex partner regular as compared to casual (Bauman \& Berman, 2005).

\section{Sexual compliance}

Sexual intercourse is not always based on mutual consent. In fact, consent to unwanted sexual activity (sexual compliance) with the aim of satisfying a partner's needs or promoting intimacy has been identified as a risk factor for acquiring STIS (O'Sullivan \& Allgeier, 1998; Vannier \& O'Sullivan, 2010). Further, contextual barriers such as coercion or manipulative behavior by a new sex partner due to gendered power differences (Melendez, Hoffman, Exner, Leu, \& Ehrhardt, 2003; Taylor, 1995) leave young women in a vulnerable position that often deters STI protection negotiation strategies.

\section{Compromised pleasure}

Although young people are generally well informed about the effectiveness of barrier protections, such as condoms, implementation of condom use behavior is still inconsistent. Reduced sexual pleasure is the most common reason for not using male condoms (L. J. Bauman, Karasz, \& Hamilton, 2007; Crosby, Milhausen, et al., 2013; Flood, 2003; Higgins \& Wang, 2015; Milhausen et al., 2018; Randolph, Pinkerton, Bogart, Cecil, \& Abramson, 2007). However, also normative factors, availability, and costs have been identified as factors compromising male condom use (Nahmias \& Nahmias, 2011).

\section{Cognitive and personality factors}

Decisions around health tend to be less preventive and more reactionary in nature (Crockett, Raffaelli, \& Shen, 2006; House, Mueller, Reininger, Brown, \& Markham, 2010; Khurana et al., 2012; Schofield, Bierman, Heinrichs, \& Nix, 2008), as young people's selfregulatory capacity is still developing and their reward-seeking behavior still pronounced (B. J. Casey, Jones, \& Somerville, 2011; Leah H. Somerville, Jones, \& Casey, 2010; Steinberg, 2008, 2010). For instance, young adults engage in more risky behaviors than adults, and it has been inferred previously that they lack optimal reasoning abilities (Arnett, 1992; Spear, 2009). This, however, turned out to be a too simplistic explanatory model, as, under optimal conditions, young adults are perfectly able to make rational decisions to achieve their goals (Gardner \& Steinberg, 2005). In fact, young adults do not have flawed reasoning capabilities or poor decision-making skills per se (Reyna \& Farley, 2006). Only in the heat of the moment with prospective instant gratification, in unfamiliar situations, in the presence of peers and when behavioral inhibition is required to achieve a good outcome, do young adults tend to reason more poorly than adults and under these 
conditions they are indeed more likely to engage in health-compromising behaviors (Reyna \& Farley, 2006). In addition, sexual arousal can affect young people's preferences and behaviors in such a way that judgment and decision making become more risk affine. As Ariely and Loewenstein (2006) showed, sexual arousal strongly influences judgment and decision making, so that gratifying but risky choices are more attractive and appealing when in an aroused state as compared to a non-aroused state. Demographic variables, personality, and individual differences can further influence behavior, however, in a more indirect way (Montaño \& Kasprzyk, 2008). While personality traits were initially defined as stable and were assumed to be the result of biological differences or situational influences - mostly early childhood experiences - (Eysenck \& Eysenck, 1985), observed low correlations in trait-related behavior across different situations have led to more complex definitions that acknowledge situational influences more strongly (Mischel \& Shoda, 1995). Risk attitude as a personality trait has undergone a similar development, as people have shown not to be consistently risk-seeking (or risk-averse) across different situations (Schoemaker, 1990). Risk taking by the same person in two situations might differ because risks and benefits are perceived differently in two domains (e.g., in a recreational vs. a personal safety decision), while the individual's attitude towards perceived risks remains the same for both domains (Weber \& Hsee, 1998; Weber \& Milliman, 1997).

Safer-sex behavior. The first step toward increasing safer-sex behavior is defining what is meant by sexual intercourse. Young adults often show variations in their views on what exactly defines sexual intercourse (Halpern-Felsher, 2008; Halpern-Felsher, Cornell, Kropp, \& Tschann, 2005), for example, only penile-vaginal - but not penile-anal - penetration is perceived as sexual intercourse, which may impact young people's ability to assess risk (Mehta, Sunner, Head, Crosby, \& Shrier, 2011). This finding is particularly alarming considering the increased risk of acquiring HIV via receptive anal intercourse (Patel et al., 2014). In fact, worldwide, only about $41 \%$ of young adults reported using a condom the last time they had sex with a new partner (World Health Organization, 2014), supporting the claim that young adults have a tendency to underestimate their risk for STIS (O'Sullivan, Udell, Montrose, Antoniello, \& Hoffman, 2010; Wolfers, de Zwart, \& Kok, 2011). Alternative preventive barrier protection methods, such as the female condom $(F C)$, remain to be unacknowledged or unaccepted by most people (Cavanaugh, Mial, \& Tulloch, 2016; Weeks, Coman, Hilario, Li, \& Abbott, 2013), maybe also due to a possible reduction of sexual pleasure (Bowling et al., 2018) and, compared to male condoms, a slightly increased financial investment (Terris-Prestholt \& Windmeijer, 2016; Witte, MacPhee, Ginsburg, \& Deshmukh, 2017). In addition, a relatively short average duration of young peoples' sexual relationships - sometimes lasting only a few weeks, adds to the problem (Henderson et al., 2002). 


\section{Reduced access to quality STI prevention services}

Compared to adults, young people are less likely to access and utilize sexual health services (Centers for Disease Control and Prevention, 2017). Such reduced access to quality STI prevention services, also due to lack of health insurance, lack of monetary resources, or general discomfort with using the available facilities, means that young people are more prone to both acquiring STIs and to infecting others (Centers for Disease Control, 2012; Hollingsworth, Anderson, \& Fraser, 2008).

Young people under the age of 30 represent over half the population of the world, and their majority resides in developing countries (Pettifor et al., 2018). This population is expected to increase markedly in the next 20-30 years (World Health Organization, 2013). Further, the transition period toward adulthood can be seen as a critical investment period with possible immediate and long-term rewards resulting from informed and evidence-based prevention efforts that can multiply across health and sociocultural domains (World Health Organization, 2001). Therefore, well-funded evidence-based prevention efforts tailored to this population (Cushman, Kantor, Schroeder, Eicher, \& Gambone, 2014) are a promising approach to ensure successful engagement and outcomes (Pettifor et al., 2018).

\section{Young people and determinants of behavior}

Social behavior has been described as a joint function of reflective and impulsive processes that interact following different operating systems, known as the dual-process approach (Strack \& Deutsch, 2004) that accounts for reasoning, judgment, and social cognition (Evans, 2008). This proposed mutual interplay is based on the distinction between deliberate - explicit - attitudes and automatic - implicit - attitudes (for a review, see Gawronski \& Bodenhausen, 2006). Impulsive precursors of a specific behavior as well as reflective precursors, and situational or dispositional conditions were suggested to enable a better prediction of self-control outcomes compared to being analyzed on their own (Hofmann, Friese, \& Strack, 2009). Even if the conceptual underpinnings of two-system models have been repeatedly challenged (e.g., Keren \& Schul, 2009), they continue to outperform other models in accounting for choices (Diederich \& Trueblood, 2018), especially related to health risk decision making as pointed out, for instance, by the prototype willingness model (for a review, see Gerrard, Gibbons, Houlihan, Stock, \& Pomery, 2008) and by a synthesis of theories about health behavior into a general dualprocess model of health-related behavior (for a review, see Houlihan, 2018). 


\section{Explicit determinants of behavior}

One explanatory model that can be used to identify determinants of intention to perform safer-sex behaviors is the Reasoned Action Approach (RAA) by Fishbein and Ajzen (2010). This approach emerged out of the Theory of Reasoned Action (TRA) (Fishbein \& Ajzen, 1975) and the Theory of Planned Behavior (TPB) (Ajzen, 1985, 1991) and suggests that behavioral decisions are made based on slow and reasoned considerations derived from available information. RAA states that behavioral intention - the readiness to engage in a behavior and hence determining the actual behavior - depends on attitude - positive or negative evaluations of behavior -, perceived norms - perceived social pressure regarding the performance or non-performance of a certain behavior -, and perceived behavioral control - PBC; perception of the ease or difficulty related to the performance of a certain behavior - (Fishbein \& Ajzen, 2010). However, high intentions to perform a certain behavior do not yet guarantee the translation of that motivation into behavior. For instance, a relationship between the intention to use condoms and actual condom use behavior has been demonstrated in multiple studies (see the meta-analysis by Albarracín, Johnson, Fishbein, \& Muellerleile, 2001). Nevertheless, it has also repeatedly been reported that the association between condom use intention and actual behavior is often weak (Bauman et al., 2007; Fridlund, Stenqvist, \& Nordvik, 2014; Sheeran \& Orbell, 1998; Smith \& de Visser, 2004; Turchik \& Gidycz, 2012). In fact, behavioral intention appears to account for just 30\% of the variance in actual behavior (Armitage \& Conner, 2001; Sheeran, 2002). This inconsistency between intention and behavior (e.g., when people with positive intentions to perform a behavior fail to act on their intentions) is referred to as "the intention-behavior gap", identifying necessary skills and a barrier-free environment as requirements to translate intentions into behavior (Sheeran, 2002). Given that intention-behavior gaps have been observed in relation to various health-promoting behaviors, including physical activity, weight loss, and illicit drug use (Sheeran, 2002; Webb \& Sheeran, 2006), it seems that a better understanding of the mechanisms that determine the strength of the link between intention and behavior is necessary. Factors outside conscious awareness that are activated automatically could provide an explanation.

\section{Implicit determinants of behavior}

Behavior is not exclusively predicted by explicit cognitive processes. Implicit associations are fast, activated automatically and can influence behavior outside conscious awareness (Gawronski, Hofmann, \& Wilbur, 2006; Greenwald \& Banaji, 1995; Hahn \& Gawronski, 2014; Rydell \& McConnell, 2006). Research has shown that implicit cognitive factors account for unique variance over and above explicit measures for a variety of behaviors, including eating behavior (Friese \& Hofmann, 2009), aggression (Wiers, Beckers, Houben, \& Hofmann, 2009), and substance use (Stacy \& Wiers, 2010; Wiers \& Stacy, 2006). 
Negative implicit cognitions also predicted less consistent condom use (e.g., Grenard, Ames, \& Stacy, 2013; Stacy, Ames, Ullman, Zogg, \& Leigh, 2006) and condom use intentions in low-risk scenarios as compared to high-risk scenarios in which intentions were better predicted by explicit attitudes (Czopp, Monteith, Zimmerman, \& Lynam, 2004). Programs aiming at changing behaviors that are recognized by young people as healthharming but are still engaged in, such as unsafe sex behavior, could be rendered more effective if automatic bases of these behaviors are specifically targeted during these programs (Grenard et al., 2008; Hofmann, Gschwendner, Friese, Wiers, \& Schmitt, 2008; Marteau, Hollands, \& Fletcher, 2012; Thush et al., 2008; for a review, see Wiers, Boelema, Nikolaou, \& Gladwin, 2015).

As laid out in protocols for the design of health promotion programs, such as Intervention Mapping (Bartholomew Eldredge, Markham, Ruiter, Fernandez, \& Parcel, 2016), health-related behavior is complex but can be broken down into different levels of comprehensive sub-behaviors. These sub-behaviors can each be targeted by tailored interventions. Therefore, identifying and understanding relevant sub-behaviors - including their determinants at different levels - is a crucial step in promoting sexual health behavior (e.g., Van Empelen \& Kok, 2006, 2008).

\section{Young people and safer-sex education programs}

School-based sex education remains important to help improve young people's sexual health. However, even if there are examples of successfully implementing school-based sex education programs, such as the Long Live Love program in the Netherlands (Mevissen et al., 2018; Schutte et al., 2014), too often are interventions well-intentioned but based on the view that sexual decision making of young people is primarily a rational and deliberate process, while leaving out the influence of affective and environmental factors as well as the cognitive, hormonal, emotional, and physical changes that accompany the transition to adulthood, while both processes are important (Ballonoff Suleiman \& Brindis, 2014; Ballonoff Suleiman, Johnson, Shirtcliff, \& Galván, 2015 )

\section{Abstinence-only and comprehensive sex education}

Both abstinence-only and comprehensive sex education programs aim to provide young people with the necessary knowledge and skills to improve and maintain their sexual and reproductive health. While abstinence-only programs focus on physical and emotional harm of casual sexual activity and strongly discourage this behavior, comprehensive education programs aim to delay the initiation of sexual intercourse, improve sexual decision making, and strengthen safer-sex behavior, such as condom use and other forms of protection against STIs (World Health Organization, 2010). Further, comprehensive 
sexuality education can also include elements focusing on positive sexual development, such as the experience of healthy, safe, and pleasurable sexual intercourse (Ballonoff Suleiman \& Brindis, 2014). Thereby, these comprehensive programs can expand sexuality education by including topics about sexual development, interpersonal relationships, body image, intimacy, and gender roles (Goldfarb \& Constantine, 2011).

Over time, abstinence-only programs have proven to be modestly effective or even ineffective at reducing sexual risk behavior in young people (e.g., Birch, White, \& Fellows, 2017; Borawski, Trapl, Lovegreen, Colabianchi, \& Block, 2005; Kohler, Manhart, \& Lafferty, 2008; Trenholm et al., 2008), and can even be counterproductive (Ott \& Santelli, 2007; Shepherd, Sly, \& Girard, 2017). Also, programs promoting monogamy were not effective at reducing sexual risk behavior (for a review, see Conley, Matsick, Moors, Ziegler, \& Rubin, 2015). In contrast, comprehensive sex education has consistently been reported to be effective at increasing safer-sex behavior of young people (e.g., Chin et al., 2012; Eisenberg, Bernat, Bearinger, \& Resnick, 2008; Kirby \& Laris, 2009; Kirby, Laris, \& Rolleri, 2007).

\section{Tailoring of sex education programs}

Combining effective, acceptable, and scalable STI preventions that address multiple key risk factors are now widely acknowledged as having the greatest combined impact on young people (Albarracín et al., 2005 ; Anderson et al., 2014 ; World Health Organization, 2017). Interventions that were identified as being effective at increasing sexual and reproductive health in young people included a long-term or repeated implementation (for a review, see Durlak \& DuPre, 2008), comprise of multiple 'active' (Albarracín et al., 2005 ) components, including parental involvement and skills-building, and were culturally, gender, and age-appropriate (for a review, see Bowring, Wright, Douglass, Gold, \& Lim, 2018). Further, it is known that gender norms (Rogow et al., 2013) and cultural factors (Schalet, 2011) influence the sexual decision-making process of young people and that including these factors in sex education programs can further improve their impact (Bell, Terzian, \& Moore, 2012). Developmentally appropriate sexuality education has also been recognized, despite the breadth of knowledge in this field, as a promising approach to tailor interventions to their populations (Silvério Marques, Goldfarb, Deardorff, \& Constantine, 2017), especially if educational differences - which are strongly associated with the age of first sexual intercourse - are taken into account while designing a comprehensive safer-sex promoting program (De Graaf, Vanwesenbeeck, \& Meijer, 2015). Tailoring education programs to their target population is costly, especially if the target population is hard-to-reach, such as socially disadvantaged groups, which presents a barrier to tailoring if programs are not adequately equipped with funding and operate within an adequate time frame (Bonevski et al., 2014). 


\section{Young people and novel prevention strategies}

The high number of young people that are every day newly infected with STIs, including HIV (UNAIDS, 2017), point to the need for novel, innovative prevention strategies (Fernández-Romero et al., 2015). In fact, tailored development of programs for young people has proven successful in lowering the high infection rate in this population (Catalano et al., 2012; World Health Organization, 2010), especially when delivered using digital technology such as internet, mobile phones, and gaming consoles (All, Nuñez Castellar, \& Looy, 2014). Integrating popular culture, such as online games, into sex education efforts - as well as responding to a need for information that integrates culturespecific expectancies - helps to ensure that programs are grounded in the lives and realities of young people (Measor, 2004; Murdock \& Kluckhohn, 1962; Rawson \& Liamputtong, 2010; Schmitt, 2005; Singer, 2010).

\section{Serious or educational games}

In recent years, digital gaming interventions have emerged as a novel way of making developmentally and culturally appropriate interventions available to a population of young people (Charsky, 2010; Enah, Moneyham, Vance, \& Childs, 2013). These so-called educational games or serious digital games are interventions delivered by computers or mobile devices that aim to educate or promote behavior change while at the same time being enjoyable and intrinsically motivating (DeSmet et al., 2014a; DeSmet, Shegog, Van Ryckeghem, Crombez, \& De Bourdeaudhuij, 2015; Graesser, Chipman, Leeming, \& Biedenbach, 2009; Pamela M. Kato, 2010; Prensky, 2007). Learning effects are fostered by utilizing immersion techniques (whereby a player becomes fully absorbed in the play), by establishing flow (a state of highly focused concentration), and by meeting the specific individual's desire to become proficient in the game (Annetta, 2010; Boyle, Connolly, Hainey, \& Boyle, 2012; Connolly, Boyle, MacArthur, Hainey, \& Boyle, 2012; Lu, Baranowski, Thompson, \& Buday, 2012). Further, gamified behavior-change interventions need some key elements to be effective, including goal setting, the possibility to overcome challenges, feedback on performance, reinforcement (gaining awards and avoiding punishments), a comparison with and monitoring of others, social connectivity, and fun and playfulness (Hightow-Weidman, Muessig, Bauermeister, LeGrand, \& Fiellin, 2017). Indeed, delivering interventions promoting behavior-change in an educational game environment has been shown to facilitate positive outcomes in participants, such as enjoyment to take part in an intervention (Hamari, Koivisto, \& Sarsa, 2014), increased learning and retention experience, but motivation was not higher compared to conventional educational methods (for a meta-analysis, see Wouters, van Nimwegen, van Oostendorp, \& van der Spek, 2013) However, in a recent meta-analysis DeSmet et al. (2014) reported that educational games failed to induce a long-term effect on behavior, 
and stressed the importance of tailoring a game not only towards behavioral outcomes but also taking into account "both socio-demographic information (e.g., age, gender, body frame) and behavioral change needs (e.g., current level of lifestyle adoption, already acquired knowledge, stages of change, or motivation)" (p.102; for a review see Boendermaker, Prins, \& Wiers, 2015). It has been recognized that this kind of tailoring of computer-based interventions can lead to engaging and immersive learning experiences that are effective in creating positive behavior outcomes, such as increased fruit and vegetable intake, and decreased calorie consumption (Starks, 2014). Tailoring game content in this way has been shown to be particularly effective in educational games for sexual health promotion (Noar, Black, \& Pierce, 2009; Portnoy, Scott-Sheldon, Johnson, \& Carey, 2008). However, the effectiveness of tailoring might also reach a limit. As DeSmet et al. (2016) pointed out in a recent meta-analysis, a participatory design (PD) approach that involves the target group in the development of a serious game either as informants or as co-designers was less effective in changing health behavior compared to an approach that integrated the target group as testers in the game. As DeSmet points out, this unexpected result could be explained by a need of substantial game design skills of future users instead of just being part of the target group, and by a need to adjust codesign techniques to the level of user design experiences. Further, as users have difficulties with relating game characteristics to learning objectives, being involved as informant results in higher effectiveness of a game than when being involved as a co-designer.

\section{An outlook on the future of serious games for young people}

At present, gamification advocates claim that digital technologies will enter the life of (not only) young people at such an extent that interventions have to be gamified if one does not want to get left behind (Cugelman, 2013). Even if the field is still in its infancy, there is indeed promising evidence suggesting that gamifying interventions can work, for instance, in e-Health applications (Sardi, Idri, \& Fernández-Alemán, 201 ā̄d) HIV prevention and care (Hightow-Weidman et al., 2017). However, a prerequisite for delivering gamified interventions is suitable technical equipment at the receiving end - the player. This means that access to a computer is the most basic need to play a serious game. As games are starting to get tailored toward mobile devices, access to smartphones and a stable and performant internet connection are also needed. This seems not to be a major problem in industrialized countries. With a yearly per capita income of $\$ 32,249.9$ in 2016 in the EU and $81 \%$ of its population having online access (World Bank, 2018), the provision of stable online networks and individual access to modern communication devices is less of a problem compared to, for instance, sub-Saharan African countries with a yearly per capita income of only $\$ 1,466.1$ in 2016 and only $20 \%$ of its population having access to the internet (World Bank, 2018), going as low as $\$ 700$ in the Central African Republic in 2016 with only $4 \%$ of its population having online access as compared to $\$ 53,600$ in the 
Netherlands in 2016 where 90\% of the population has online access (World Bank, 2018). However, the vast majority of people living with HIV in 2016 are located in low- and middle-income countries, with an estimated 25.5 million living in sub-Saharan Africa (UNAIDS, 2017), where three in four new HIV infections occur in young people aged between 15 and 19 years (UNICEF, 2017). Given this structural discrepancy, it is most likely that in the next years mostly young people living in higher-income countries will have the opportunity to increase their abilities in preventing STIs or HIV infection by serious gaming interventions.

\section{Research objectives and outline of the dissertation}

The overall aim of this doctoral thesis is to report on a novel approach to increase safersex behavior in young people by a serious game approach. Most sex education programs as well as research on sexual risk behavior is focused on the use of barrier protection, such as condoms. For this project, we broaden this focus by adding to condom use the gathering of information regarding a new sex partner's sexual health and the refraining from risky sexual practices, such as penetrative anal sex. These three sexual health protecting behaviors have been identified to reduce the risk of acquiring STIs (Varghese, Maher, Peterman, Branson, \& Steketee, 2002). Specifically, the relative risk of HIV infection is reduced 47 -fold by choosing a sex partner who has tested negative (as compared to an untested sex partner). Using condoms further reduces the risk 20-fold; and abstinence from penetrative anal sex in favor of penetrative fellatio reduces the risk 13-fold (Kim \& Koopman, 2012; Varghese et al., 2002). The serious game that was designed and programmed for this project aimed at increasing the implementation of these three protective behaviors in young people, aged between 16 and 24 years.

In the first chapter, we tested whether distal variables could be additional predictors of the three safer sex behaviors of interest in young people. This study extended the RAAguided prediction model by including the following distal variables, in addition to assessing past behavior (i.e., protective measures taken during last sexual intercourse): behavioral inhibition, sensation seeking, parental monitoring, and knowledge of STIs and their transmission.

The study presented in the second chapter was the second part of the study described in chapter one. This part, however, was designed to allow young adults, aged 18-24 years, to express their attitudes and perceptions about safer-sex measures, without being limited by predefined answers, in an online questionnaire consisting of open-ended questions about the reasons behind why they do or do not (1) engage in less risky sex acts, (2) ask a new sex partner about his/her sexual health status, and (3) use condoms during sexual intercourse. We then combined variables derived from the Reasoned Action Approach of the study described in the first chapter with questions about participants past protective behavior, STI and HIV testing history, and current estimation of 
sexual health. Finally, we analyzed which factors encourage young adults to carry out these three protective behaviors or prevent them from doing so.

In chapter three we present a study that aimed to identify content- and design-related preferences among future players of the educational game promoting safer-sex that we were developing at that time for young people. Furthermore, we wanted to investigate whether these game-related preferences would differ depending on gender and cultural background. Therefore, we focused on identifying potential game-related content and design preferences of future players. Further, we explored whether these game-related preferences would differ depending on the gender and cultural background (German or Turkish) of the players.

The fourth chapter is aimed to get more insight in how young people's explicit and implicit cognitions towards the three safer-sex behaviors of interest as well as toward more general health protecting behaviors might differ depending on their level of sexual arousal. More specifically, we were interested if predictors of the three safer-sex behaviors differed dependent on the participants' state level of sexual arousal (sexually aroused versus not sexually aroused). One of our questions was if measures of explicit and implicit determinants of sexual decision-making were influenced by sexual arousal. Second, we were interested if working memory capacity, as a proxy for executive functions, is affected by sexual arousal. Third, we were interested if the assessment of other factors with possible long-term consequences, such as risk perception, health behavior, or life expectancies is affected by sexual arousal. Fourth, because of the widespread use of the RAA in predicting and changing behavior, we were interested if the predictive power of the main determinants of behavioral intention advocated by this model - namely attitude, social norms, and perceived behavioral control - was altered depending on the level of sexual arousal.

In chapter five we tested if having high intentions to use condoms is sufficient for translation into behavior. Therefore, we used electroencephalography (EEG) and adapted an experimental paradigm previously used by (Kessels, Ruiter, \& Jansma, 2010) to examine whether negotiation regarding condom use induces an early attention disengagement process related to embarrassment. We expected that pictures depicting condom negotiation would induce embarrassment and that individuals with high intention to use condoms but with inconsistent (past) condom use behavior (intention-behavior inconsistent individuals), as compared to those with high intention to use condoms and consistent (past) condom use behavior (intention-behavior consistent individuals), would attempt to reduce their feeling of dissonance by disengaging attention from those pictures. We expected that intention-behavior inconsistent participants would make use of fewer attentional resources to detect visual targets as compared to individuals for whom the information was less self-relevant (i.e., participants with high intention and consistent (past) condom use behavior). We also expected participants with intentionbehavior inconsistency to show lower P300 amplitudes (an event-related potential 
known in EEG studies to reflect the use of attentional resources) and faster reaction times than intention-behavior consistent participants.

In chapter six we report the effectiveness of the developed serious game to increase young people's intentions to implement the three safer-sex precautions of interest. Therefore, we tested if behavior change techniques, such as evaluative conditioning (EC; Bar-Anan, De Houwer, \& Nosek, 2012), task-relevant response inhibition training (selective RI; Allom, Mullan, \& Hagger, 2016; Eagle, Bari, \& Robbins, 2008), and working memory training (WM; Klingberg, 2010), presented in a serious game would strengthen explicit behavioral determinants (particularly behavioral intention) and implicit attitudes towards implementing the three protective behaviors. More precisely, before playing the serious game, participant's explicit behavioral determinants (measured by RAA variables) and implicit associations towards the three protective behaviors were assessed. Participants were then randomly assigned to different conditions (consisting of various combinations of active and mock versions of EC, task-relevant RI training, and WM training, and of different numbers of playing sessions). After the intervention, participants' explicit behavioral determinants and implicit associations toward the three protective behaviors were reassessed. We predicted that, first, mock only conditions would not result in any significant differences between pre- and post-intervention measures. Second, we predicted that a combination of active versions of EC, task-relevant RI training, and WM training would lead to stronger explicit determinants and implicit attitudes toward the three protective behaviors compared to any other combination of active and mock versions. Third, we predicted that participants who played five sessions would show stronger explicit behavioral determinants and implicit attitudes toward the three protective behaviors compared to participants who played only three sessions. Further, participants who played three sessions would show stronger explicit and implicit determinants than participants who only played the game once.

In the final chapter, chapter seven, the findings described in the previous chapters are summarized, reflected, integrated, and discussed. This chapter further addresses the implications of the conducted studies and proposes suggestions for future research.

This work was supported by a grant from Aids Fonds (2011034). All studies were approved by the institutional ethics review board of the Faculty of Psychology and Neuroscience at Maastricht University. 



\section{Chapter One}

\section{Identifying Psychosocial Variables that Predict Safer-sex Intentions in Adolescents and Young Adults}

Based on:

Brüll, P., Ruiter, R. A. C., Wiers, R. W., \& Kok, G. (2016). Identifying Psychosocial

VariablesThat Predict safer sex intentions in adolescents and Young adults. Frontiers in Public Health, 4. http://doi.org/10.3389/fpubh.2016.00074 


\section{Abstract}

Young people are especially vulnerable to sexually transmitted infections. The triad of deliberate and effective safer-sex behavior encompasses condom use, combined with additional information about a partner's sexual health, and the kind of sex acts usually performed. To identify psychosocial predictors of young people's intentions to have safer sex, as related to this triad we conducted an online study with 211 sexually active participants aged between 18 and 24 years. Predictors (i.e., perceived behavioral control, subjective norms, and intention) taken from Fishbein and Ajzen's Reasoned Action Approach (RAA), were combined with more distal variables (e.g., behavioral inhibition, sensation seeking, parental monitoring, and knowledge about sexually transmitted infections). Beyond the highly predictive power of RAA variables, additional variance was explained by the number of instances of unprotected sexual intercourse during the last twelve months and reasons for using barrier protection during first sexual intercourse. In particular, past condom nonuse behavior moderated perceived behavioral control related to intended condom use. Further, various distal variables showed significant univariate associations with intentions related to the three behaviors of interest. It may, therefore, be helpful to include measures of past behavior as well as certain additional distal variables in future safer-sex programs designed to promote health sustaining sexual behavior. 


\section{Introduction}

Each year, an estimated half a billion new curable sexually transmitted infections (STIs) occur worldwide (World Health Organization, 2013), with young people aged 15-24 years (Sawyer et al., 2012) acquiring nearly half of them (Centers for Disease Control, 2012). Reduced access to quality STI prevention services (e.g., due to lack of health insurance, lack of monetary resources, discomfort with using the available facilities) mean that young people are more prone to both acquiring STIs and to infecting others (Centers for Disease Control, 2012; Hollingsworth et al., 2008). Another factor which makes young people especially vulnerable to sexually transmitted infections is their often widely divergent views on what exactly defines sexual intercourse (Halpern-Felsher, 2008; HalpernFelsher et al., 2005). For instance, some young people only define penile-vaginal - but not penile-anal - penetration as sexual intercourse (Mehta et al., 2011). This finding is particularly alarming in light of the increased risk (as compared with previous estimates) of acquiring human immunodeficiency virus (HIV) via receptive anal intercourse (P. Patel et al., 2014b). In addition, the relatively short average duration of young peoples' sexual relationships particularly those aged $15-19$ years, whose relationships tend to last only a few weeks, adds to the problem (Henderson et al., 2002). To complicate matters even further, young people tend to show a discrepancy between, on the one hand, an increase in reward-seeking behavior, and at the same time, an under-developed capacity for effective self-regulation in risky situations (Casey et al., 2011; Somerville et al., 2010; Steinberg, 2008, 2010).

According to WHO data, only about $41 \%$ of young people with multiple partners reported using condoms the last time they had sex (World Health Organization, 2014). As Nahmias and Nahmias (2011) have pointed out, safer-sex behavior is complex. It includes sub-behaviors such as evolved patterns of sexual partnering and promiscuity in increasingly heterogeneous sexual networks that are associated with travel, sometimes on a global scale. Therefore, sub-behaviors - as well as normative factors, availability, and costs - need to be addressed in the development of programs designed to promote safersex.

Encouraging the use of safe sex sub-behaviors could be a promising approach when it comes to the reduction of HIV infection rates. Specifically, the relative risk of HIV infection is reduced 47 -fold by choosing a sex partner who has tested negative (as compared to an untested sex partner). Using condoms further reduces the risk 20-fold; and abstinence from penetrative anal sex in favor of penetrative fellatio reduces the risk 13 -fold (Kim \& Koopman, 2012; Varghese et al., 2002).

As laid out in protocols for the design of health promotion programs such as Intervention Mapping (Bartholomew Eldredge et al., 2016), health-related behavior is complex in nature. However, it can be broken down into different levels of comprehensive sub-behaviors that can each be targeted by tailored interventions. Identifying and 
understanding relevant sub-behaviors - including their determinants at different levels is a crucial step in promoting health sustaining behavior.

One explanatory model that can be used to identify determinants of intention to perform safer-sex behaviors is the Reasoned Action Approach (RAA) (Fishbein \& Ajzen, 2010). This approach evolved from the Theory of Reasoned Action (TRA) (Fishbein \& Ajzen, 1975) and the Theory of Planned Behavior (TPB) (Ajzen, 1985, 1991). These models of human behavior suggest that behavioral decisions are made based on reasoned considerations derived from available information. RAA states that behavioral intention - defined as the readiness to engage in a behavior and hence determining the actual behavior - depends on attitude, perceived norms (PN), and perceived behavioral control (PBC) (Fishbein \& Ajzen, 2010). Attitudes are defined as positive or negative evaluations of behavior. Perceived norms are defined as perceived social pressure regarding the performance or non-performance of a certain behavior, and perceived behavioral control is defined as perception of the ease or difficulty related to the performance of a certain behavior.

With regard to explaining risky behavior, it has been observed that young people are more likely to engage in riskier behaviors compared to older age groups (e.g., Arnett, 1992; Spear, 2009). However, it has been convincingly argued that young people do not have flawed reasoning capabilities or poor decision-making skills per se. Under ideal conditions, they have an almost perfect ability to make rational decisions in order to achieve their goals (see Crone \& Dahl, 2012; Gardner \& Steinberg, 2005). It is only in the heat of the moment, in unfamiliar situations, in the presence of peers and when behavioral inhibition is required to achieve a health sustaining outcome that young people tend to reason more poorly than adults (Reyna \& Farley, 2006; Steinberg, 2008, 2010, 2011).

Moreover, according to Montaño and Kasprzyk (2008), demographic variables, personality, and individual differences "may be associated with behaviors, but their influence is indirect" (p.81). To test whether distal variables could be additional predictors of safer sex behavior, this study extended the RAA-guided prediction model by including the following distal variables, in addition to assessing past behavior (i.e., protective measures taken during last sexual intercourse):

- Behavioral inhibition: Sexual gratification is closely related to human pleasure and strongly influences human behavior (Birnbaum, Mikulincer, Szepsenwol, Shaver, \& Mizrahi, 2014). Being able to inhibit the promise of pleasure that is potentially health-compromising is an essential component of health sustaining behavior (e.g., Ajzen \& Driver, 1991).

- Sensation seeking: Zuckerman (1971) defined sensation seeking as a personality trait characterized by "the need for varied, novel and complex sensations and experiences, and the willingness to take physical and social risks for the sake of such experiences" (p.10). Adolescent risk behaviors have consistently been linked to 
sensation seeking (e.g., Greene et al., 2000), making this trait a possible predictor of health-compromising sexual behavior.

- Parental monitoring: Parenting has been closely linked to late adolescent (15-19 years) sexual risk behaviors (e.g., Kincaid, Jones, Sterrett, \& McKee, 2012; Sieverding, Adler, Witt, \& Ellen, 2005). However, less is known about the predictive power of parenting as related to intentions to: ask a sex partner about his/her health status, perform less risky sex acts, or use condoms during next sexual intercourse.

- Knowledge of STIS and their transmission: Knowledge about STIs and knowledge of HIV prevention varies widely within the 15-19 year-old age group, and there are no reliable data (including most Western European countries) currently available on this topic (Avery \& Lazdane, 2010). However, having knowledge about STIs and their prevention is a key precondition to health-sustaining sexual behavior (World Health Organization, 2014).

Based on the per-act risk of acquiring HIV (Kim \& Koopman, 2012; P. Patel et al., 2014b; Varghese et al., 2002), and on the fact that adolescents often underestimate their risk of acquiring STIs (e.g., O'Sullivan et al., 2010; Wolfers et al., 2011), we included the following specific sub-behaviors, and looked for variables predicting the intention to: (1) ask a sex partner about his/her health status, (2) perform less risky sex acts, and (3) use condoms when next having sexual intercourse with a new partner. Using this framework, we expect that (a) in line with RAA, perceived norms and perceived behavioral control will predict the intention to perform each aforementioned safer-sex sub-behavior, and that (b) according to Montaño and Kasprzyk (2008), distal, social, and past behavior variables will have indirect effects on the intention to perform each of the three aforementioned safersex precautions through more proximal RAA variables. Due to a technical problem during data collection, it was not possible to measure attitudes towards target behaviors 1-3 (see section on limitations).

\section{Material and Methods}

\section{Participants and procedure}

Participants aged between 18 and 24 years who were not in a relationship (where safersex precautions might not play such a vital role) at the time of the survey were recruited from a German online panel. This panel specializes in social and academic research and offers the option of selecting participants according to their age and demographics (www.meineumfrage.com). Participants were linked to a secured website that contained the study materials. An introductory page explained the aim of the study, namely to find out a possible connection between conscious and unconscious factors in the process of 
decision making concerning safer-sex behavior. Participants were rewarded with panelowned credit points that could be exchanged for money. Furthermore, if they wished to do so, participants could enter a lottery in order to win one of three additional online shopping credit vouchers (€50,- each). Anonymity was assured as e-mail addresses were not linked to individual data. Completing the questionnaire took approximately 25 to 30 minutes. A pretest showed that the survey could be completed within 20 minutes time. The survey allowed for breaks according to individual needs. Completed questionnaires were anonymously returned to the experimenter's e-mail inbox in such a way that it was not possible to link the panel issued e-mail to the identity of a participant.

Of the 1114 questionnaires that were accessed online, 242 were completed and returned $(21.7 \%)$. This high drop-out rate is not unusual for this particular panel because participants are encouraged to visit the sites containing study materials and only then to decide whether to participate or not. Of course, visiting the website out of curiosity is counted as access but is only rewarded with panel-owned credit points after completion of the survey. Thirty-one participants were excluded because they did not answer more than $90 \%$ of all required questions. This left a sample of 211 participants for analysis.

\section{Measures}

Demographic characteristics and past sexual experience were assessed by asking participants about sex, age, size of their place of residence (big city: equal or more than 1 million inhabitants, city: between 10,000 and 1 million inhabitants, rural area: less than 10,000 inhabitants), and composition of household (living with parents/family, alone, shared apartment). Questions about past sexual behavior (Peterman et al., 2000) assessed sexual identity (e.g., Centers for Disease Control, 2011), age at the time of their first sexual intercourse, age of their sex partner at the time of their first sexual intercourse, relationship status with their sex partner at the time of their first sexual intercourse (i.e., met shortly before, superficial friendship, close friendship, loose relationship, close relationship, married), barrier protection against STIs during their first sexual intercourse, number of different sex partners during the last 6 months, number of instances of unprotected sexual intercourse (SI) during the last twelve months, and an estimation of whether they might currently have an STI. Furthermore, participants were asked if they had ever taken an HIV test, and whether they had been tested for STIs during the last twelve months.

\section{Distal variables}

\section{Behavioral inhibition}

Eight items (adapted from Carver \& White, 1994; Patton, Stanford, \& Barratt, 1995) using a 5-point Likert-Scale ranging from "always" to "never" (e.g., "I plan tasks accurately.") were used to measure behavioral inhibition. Cronbach's alpha, based on the mean inter- 
item correlation (a Cronbach's alpha of greater than .60 is satisfactory, and a Cronbach's alpha of greater than 80 is good), was $\alpha=.32$. Correlations between single scale items with outcome variables were also low. The scale was adapted from Barratt's Behavior Inhibition Scale (Patton et al., 1995) that in its original form contains 30 items. Due to web-related time and space requirements, only eight representative items were selected for this questionnaire. This relatively small number of selected items may have resulted in a lack of scale reliability. Despite Kline (1993) arguing that lower Cronbach's alpha values can be expected for psychological constructs, and Nunnally and Bernstein (1994) proposing that values as low as .50 are sufficient to identify a scale as reliable, we nevertheless discarded the behavioral inhibition data from our analysis based on these results.

\section{Sensation seeking}

Sensation seeking was measured with eight items (Hoyle, Stephenson, Palmgreen, Lorch, \& Donohew, 2002) (e.g., "I like wild parties.") using a 5-point Likert-Scale ranging from "completely disagree" to "completely agree"; $\alpha=.73$.

\section{Parental monitoring}

How well parents or legal guardians were informed about the social life of participants during their late adolescent years was measured by 10 items (adapted from Beyers \& Goossens, 1999) (e.g.," How well did your parents or legal guardians know the parents or legal guardians of your close friends?" or "How well did your parents or legal guardians know about your activities when you were not at home?") using a 7-point Likert-Scale ranging from "not at all" to "very well." Cronbach's alpha for these measurements was $\alpha$ $=.82$.

\section{Knowledge}

Participants' knowledge about STIs and safer sex practices was indexed by means of 15 true-false questions (e.g., "If a 14-year-old teenager has been recently infected with HIV, the first AIDS-related symptoms could appear as late as in his/her mid-twenties."). Participants were directed not to guess, and instead to use the "I am not sure" response option when necessary. A correct answer was given +1 point, and an incorrect answer or an "I am not sure" answer was given 0 points. The sum score for knowledge ranged between 0 and 15 points.

\section{RAA variables}

The RAA variables were all measured using 7-point Likert scales ranging from 1 = completely agree to $7=$ complely disagree. Scores on items that were included to assess the same variable were averaged into one measure in cases where internal consistency was sufficient (Cronbach's alpha $>.60$ or Pearson $r>.30$ with two items). Items were recorded such that higher scores reflect a stronger presence of the variable concerned. 
Intention to ask a sex partner about his/her health status (iHS). One item assessed the participants' intention to ask his/her sex partner about his/her health status before s/he would next have sexual intercourse: "I intend to ask my new sex partner about his/her health status before I next have sex with him/her."

Perceived behavioral control related to asking a sex partner about his/her health status (pbchS). Two items ("I am confident to ask my new sex partner about his/her health status before I next have sex" and "Whether I inform myself about my new sex partner's health status before I next have sex with him/her is up to me") measured perceived behavioral control related to asking a sex partner about his/her health status $(r=.34)$.

Subjective norm towards asking a sex partner about his/her health status (pnHS). The subjective norm related to asking a sex partner about his/her health status was measured by the following two items ( $r=.62$ "Most people who are important to me think I should ask my new sex partner about his/her health status before I next have sex with him/her" and "Most people like me think I should ask my new sex partner about his/her health status before I next have sex with him/her".

Intention to perform less risky sex acts (iSA). The item "I intend to perform less risky acts when I have sex with a new partner for the next time" assessed the participants' intention to perform less risky sex acts (e.g., insertive fellatio instead of insertive anal sex) during their next sexual intercourse.

Perceived behavioral control related to performing less risky sex acts (pbcSA). Two items (i.e., "I am confident that I will perform less risky sex acts when I next have sex with a new partner" and "It is up to me whether I perform less risky sex acts when I next have sex with a new partner") measured perceived behavioral control related to performing less risky sex acts when next having sexual intercourse; $r=.37$.

Subjective norm towards performing less risky sex acts (pnSA). The subjective norm related to performing less risky sex acts was measured by the following two items ( $r=.66$ ): "Most people who are important to me think I should perform less risky sex acts when I next have sex with a new partner" and "Most people like me think I should perform less risky sex acts when I next have sex with a new partner."

Intention to use condoms (iCU). The item "I intend to use condoms when I next have sex with a new partner" assessed the participants' intention to use condoms when next having sexual intercourse.

Perceived behavioral control related to using condoms (pbcCU). Two items ("I am confident that I will use condoms when I next have sex with a new partner" and "It is up to me whether I use condoms when I next have sex with a new partner") measured participants' perceived behavioral control regarding the use of condom when next having sexual intercourse with a new partner; $r=.53$. 
Subjective norm towards condom use (pnCU). The subjective norm related to condom use was measured by the following two items ( $r=.61$ ): "Most people who are important to me think I should use condoms when I next have sex with a new partner" and "Most people like me think I should use condoms when I next have sex with a new partner."

\section{Statistical analysis}

Data were analyzed using the predictive analytic computer software SPSS, version 22.0 (Statistical Product and Service Solutions, IBM, New York). Following a descriptive analysis, the univariate association between all study variables was analyzed with Pearson correlation coefficients (Table 2). Variables that showed significant associations with intention (i.e., asking a partner about his/her health status, performing less risky sex acts, and using condoms the next time they would have sexual intercourse) were entered into a hierarchical multiple regression to assess both their unique contribution towards the explanation of intention and the total amount of variance in intention explained by the model. To test for indirect effects of distal variables on intention variables (e.g., perceived behavioral control) we conducted a hierarchical stepwise regression in which variables were entered from the linear equation on the basis of their ability to improve $R^{2}$ at each successive step. Furthermore, moderation analysis was conducted to test whether distal variables (e.g., sensation seeking) influenced the strength of the associations between RAA and intention variables. First, each predictor was centralized; the moderator was entered into the model, and finally, the interaction of these two was entered. If the interaction term was significant after controlling for direct effects of predictor and moderator, the moderation was considered significant. Subsequently, simple effect analysis was conducted to determine the influence of distal variables on the associations of RAA variables with intention. Results were considered significant at $p<.05$.

\section{Results}

\section{Demographics}

The majority of the respondents were male (5 $4 \%$, n= 1144$)$, with a mean age of 23.5 years. The mean age of female participants was 2.8 years (4 $6 \%, n=97$ ). Of all participants, $43 \%$ ( $n=9 \quad 1$ ) were living in a big city of 1 million or mbabitants whereas $38 \%(n=8 \quad 1$ ) were living in a city of between 10,000 and 1 million habitants, and $18 \%(n=39)$ were living in rural areas of less than 10,000 habitants. Most participants (7 $3 \%, n=154$ ) were living in shared apartments while 17 (8\%) were living alone and 40 (19\%) were living with their parents or family. All participants ( $\left.n=2 \begin{array}{lll}2 & 1 & 1\end{array}\right)$ classified themselves as heterosexual. Past sexual experiences grouped by sex are reported in Table 1. 
Table 1. Past sexual experiences grouped by sex.

\begin{tabular}{|c|c|c|}
\hline & Male & Female \\
\hline Age in years of first sexual intercourse & $M=17.6 \quad(S D=2.64)$ & $M=16.9 \quad(S D=2.17)$ \\
\hline Age in years of first sex partner & $M=18.8 \quad(S D=4.05)$ & $M=19.8 \quad(S D=3.53)$ \\
\hline \multicolumn{3}{|l|}{ Relationship to first sex partner } \\
\hline - met shortly before intercourse & $28 \%(N=32)$ & $14 \%(N=14)$ \\
\hline - already befriended & $55 \%(N=63)$ & $27 \%(N=26)$ \\
\hline - started relationship & $33 \%(N=38)$ & $59 \%(N=57)$ \\
\hline Used barrier protection during first intercourse & $67 \%(N=76)$ & $77 \%(N=75)$ \\
\hline $\mathrm{N}$ different sex partners (past 6 months) ${ }^{*}$ & \multirow{2}{*}{\multicolumn{2}{|c|}{ 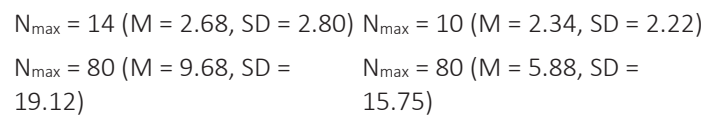 }} \\
\hline $\begin{array}{l}\mathrm{N} \text { instances unprotected sex (past twelve } \\
\text { months) }{ }^{*}\end{array}$ & & \\
\hline HIV test taken at least once in lifetime & $57 \%(N=65)$ & $49 \%(N=48)$ \\
\hline STI test taken during past twelve months & $12 \%(N=14)$ & $22 \%(N=22)$ \\
\hline Estimation of not having an STI & $92 \%(N=105)$ & $94 \%(N=91)$ \\
\hline
\end{tabular}

*numbers estimated by participants

\section{Factorial analysis}

A principal component analysis was conducted for each distal variable. Factor analysis allowed us to identify components with eigenvalues greater than 1.00 that could be grouped together in order to form new factors. All factor structures were obtained by orthogonal rotation. Only factors with eigenvalues equal to or greater than 1.00 were extracted. A new factor apparently related to "being impulsive" explained $28 \%$ of the variance ( $\alpha=.58$ ), a second factor related to "being anticipatory" accounted for $18 \%$ of variance ( $\alpha=.61$ ( 1 ), and a third factor related to "being analytikaexplained $14.5 \%$ of the variance ( $\alpha=.59$ ). Factorial analysis of "sensation seeking" resulted in a new factor related to "risk-seeking" ( $\alpha=.72$ ) that explained $49 \%$ of the variance. A second factor seemingly related to "improvising" ( $\alpha=.70$ ) accountedif $14 \%$ of variance. Parental monitoring could be disentangled into three new factors. "Parental knowledge about the activities of participants during the time they were unattended" explained $45 \%$ of the variance $(\alpha=.90$ ). A second factor related to "breakg parental rules" accounted for $14 \%$ of variance ( $\alpha=.42$ ), and lastly, parental "decision power" accounted for 13 Pariance ( $\alpha$ $=.7 \quad 3$ ).

\section{Univariate associations}

Table 2 presents the Pearson correlations among the study variables with $r=.1$-.03 indicating a small effect, $r=.24 .36$ indicating a moderate effect, and $r \geq .37$ indicating a large effect (Cohen, 1988, 1992; Cohen, Cohen, West, \& Aiken, 2003).

Positive univariate associations with intention. Strong positive associations were found between the RAA variables subjective norm and perceived behavioral control and 
intention for each of the three target behaviors ( $r$ 's $>$.198). Concerning the distal variables, small significant univariate associations with intention to ask a new sex partner about his/her health status were found for the number of instances of unprotected sexual intercourse during the last twelve months $\left(r=. \begin{array}{lll}1 & 4 & 1\end{array}\right)$, for the status of relationship with the first sex partner $\left(r=. \begin{array}{lll}1 & 4 & 2\end{array}\right)$, and farmental knowledge about activities during unsupervised time ( $\left.r=\begin{array}{lll}1 & 7 & 0\end{array}\right)$. No significant association was identified between intention to refrain from risky sex practices and distal variables. Small significant univariate associations with intention to use condoms during first time sex with a new partner were found for parental decision power ( $r=.146$ ), for knowledge about STIs ( $r=.164$ ), and for the age of first sexual intercourse ( $r=.191$ ). No moderate and large associatiamere identified between the distal variables and the three intention measures.

Negative univariate associations with intention. Small significant univariate associations with intention to ask a new sex partner about his/her health status were found for having been tested for STIs in the past twelve months ( $r=-.147$ ) and for being analytical ( $r=$ .177). No moderate and large effects were identified. Small significant univariate associations with intention to refrain from risky sex practices were found for risk-seeking $(r=-$ .142), for barrier protection during first sexual intercourse $(r=-.146)$, and for the number of different sex partners in the past six months $(r=-.186)$. No moderate and large effects were identified. Small significant univariate associations with intention to use condoms during first time sex with a new sex partner were found for reasons to protect during the first sexual intercourse $(r=-.150)$ and for the status of relationship with the first sex partner $(r=-.152)$. Moderate significant univariate associations with the intention of condom use were found for the number of instances of unprotected sexual intercourse during the last twelve months $(r=-.301)$ and for protection during first sexual intercourse $(r=-.325)$. No other significant associations were found between the distal variables and the three intention measures. 


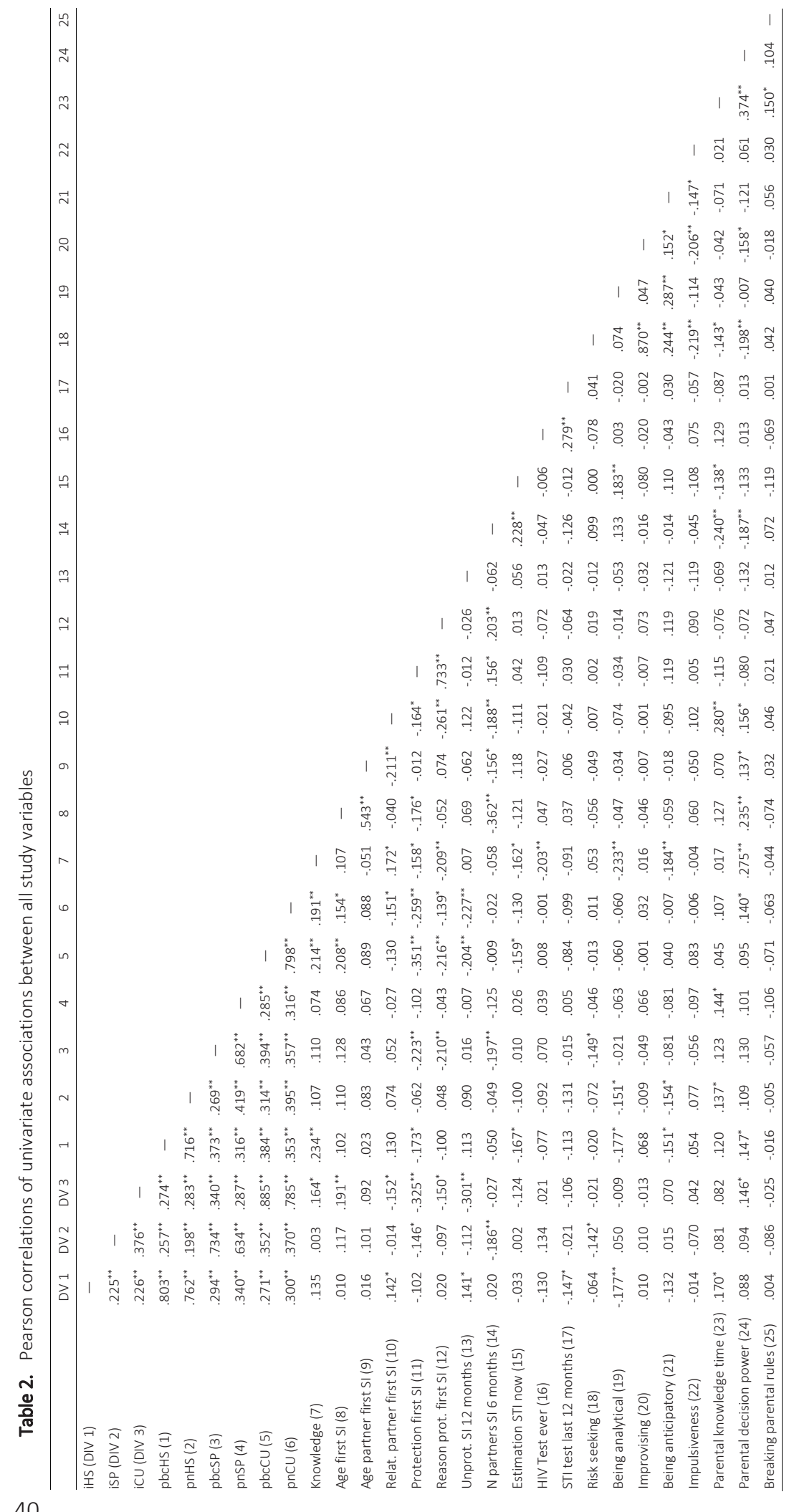


Identifying Psychosocial Variables that Predict Safer-sex Intentions

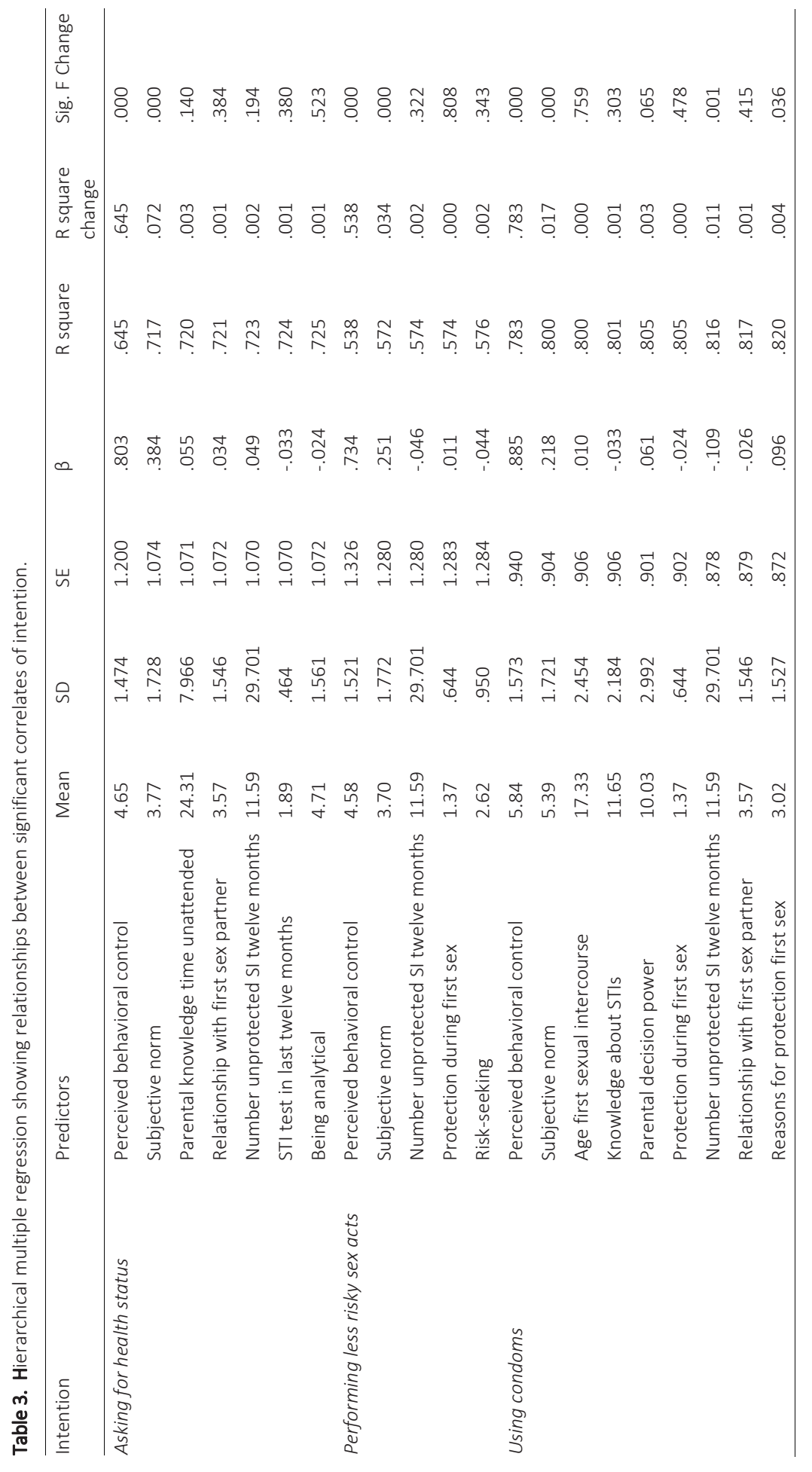




\section{Regression analysis}

We used hierarchical multiple regression to explore relationships between significant correlates of intention. The variance inflation factor (VIF) for all variables remained below 3 so that no multicollinearity was detected (a VIF greater than 10 indicates multicollinearity, see Bowerman \& O'Connell, 1990; Myers, 1990). Table 3 shows mean, standard deviation, $R^{2}$ and $R^{2}$ change and centralized regression coefficients (betas). In relation to intention to ask a sex partner about his/her health status, the RAA predictors perceived behavioral control, and subjective norms accounted for $71.7 \%$ of variance (perceived behavioral control accounted for $64.5 \%$ and subjective norms for an additional $7.2 \%)$. Distal predictors added no significant explanation of the variance.

Regarding the intention to perform less risky sex acts, RAA predictors perceived behavioral control and subjective norms accounted for $57.2 \%$ of variance (perceived behavioral control accounted for 53.8\%, and subjective norm accounted for an additional 3.4\%). Distal predictors added no significant explanation of the variance.

Concerning the intention to use condoms, RAA predictors perceived behavioral control, and subjective norms accounted for $80 \%$ of variance (perceived behavioral control for $78.3 \%$ and subjective norms for an additional $1.7 \%$ ). The number of instances of unprotected sexual intercourse during the last twelve months explained an additional $1.1 \%$ of variance. This was followed by reasons to use barrier protection during first sexual intercourse which explained a further $0.4 \%$ of variance.

\section{Moderation analysis}

Next, we conducted a moderation analysis to test if distal variables moderated the relationship between RAA predictor variables and intentions. Only regarding the intention to use condoms, results showed a significant interaction effect between the number of instances of unprotected sexual intercourse during the last twelve months and perceived

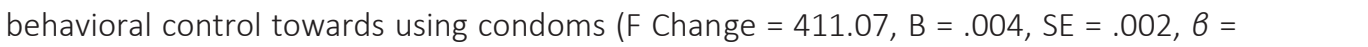
$.082, p=.017$ ).

Simple slope analyses (see Aiken \& West, 1991) for the association between perceived behavioral control to use condoms and actual condom use intention were tested for a low (-1 SD below the mean) and a high ( +1 SD above the mean) number of instances of unprotected sexual intercourse during the last twelve months. Each of the simple slope tests revealed a significant association between perceived behavioral control to use condoms and intention to use condoms, but perceived behavioral control was more strongly related to condom use intention for a high number of past instances of unprotected sexual intercourse $(B=1.20, \mathrm{SE}=. \theta=6.9,4 p,<.0 \quad 0 \quad 1)$ than for a low number $(\mathrm{B}=.98, \mathrm{SE}=$ $.06, b=.76 p,<.001)$. Figure 1 plots the simple slopes for the interaction. 


\section{Number of unprotected SI moderates CU PBC significantly}

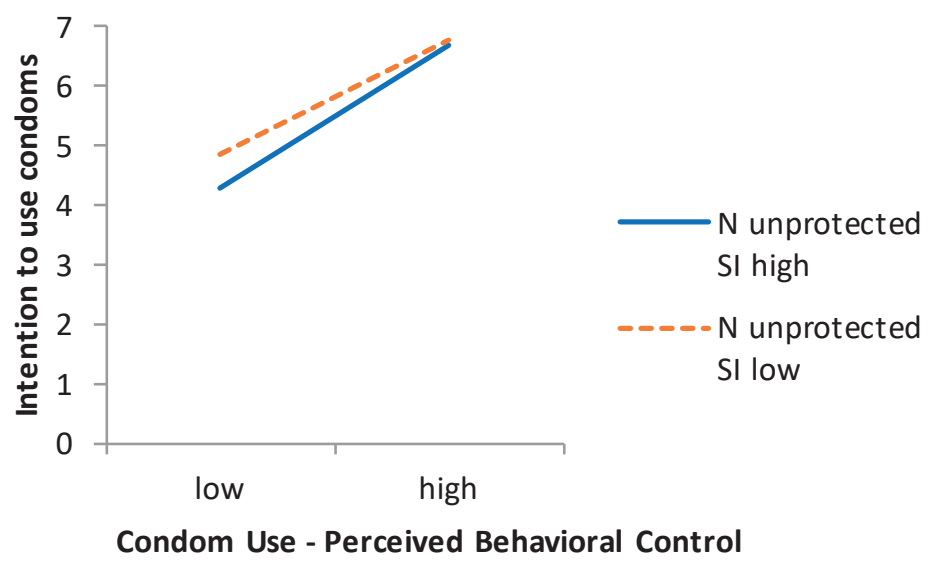

Figure 1. Regression slopes arising from the relation between perceived behavioral control towards condom use and intention to use condoms with a new sex partner for participants who are relatively high (one SD [SD = 1.57] greater than the mean, see Aiken \& West, 1991) versus low (one SD less than the mean) in the number of instances of unprotected sexual intercourse in the past.

\section{Discussion}

Perceived behavioral control and subjective norms predicted participants' intention to perform safer sexual behavior - by up to 80 的 relation to condom use. Both of these RAA variables also predicted intention to ask a partner about his/her health status (accounting for $71.7 \%$ of variance), as well as intention to perform less risky sex acts (57.2\%). Although these values may appear high, according to Fishbein and Ajzen (2010), such percentages are not uncommon if, as in this study, the correspondence principle is strictly followed by taking into account factors such as time, action, target, and context. This primacy of RAA variables over distal variables is further accentuated by the fact that analyses of attitude measures could not be included due to a technical problem during data acquisition. RAA variables emerged as the most prominent predictors of intentions related to the safer-sex behaviors investigated in this study. The intention to perform less risky sex acts and to ask a new sex partner about his/her health status was low compared to the intention to use condoms. Only $13.3 \%$ of all participants reported that they intended to perform less risky sex acts, and only $15.2 \%$ intended to ask a new sex partner about his/her health status, whereas $56.9 \%$ intended to use condoms the next time they would have sex with a new partner. 


\section{Intention to ask a new sex partner about his/her health status}

Perceived behavioral control (64.5\%) and subjective norm (7.2\%) were the main predictors of intention to ask a new sex partner about his/her health status. However, intention was low. In fact, even if scores of participants who strongly intended to ask a new sex partner about his/her health status were combined (points 6 and 7 of the 7-point Likertscale measuring intention), only a total of $23.3 \%$ of participants intended to perform this behavior. Even if distal variables did not predict intention, parental monitoring during adolescence showed a significantly though small positive univariate association with both intentions to ask a new sex partner about his/her health status, and to use condoms during their first sexual intercourse with a new partner. This finding is in line with Bronfenbrenner's (1979) model that accords parents a direct influence on their child. Furthermore, an authoritative parenting style with balanced support and monitoring (Baumrind, 1978) has been identified as one which promotes optimal child outcomes (e.g., Jones et al., 2005; Sterrett, Jones, \& Kincaid, 2009). Monitoring not only restricts adolescents' opportunities to engage in risky behavior (Sieverding et al., 2005), but is also a factor associated both with caring about safer-sex practices by asking about the health status of a new sex partner and with condom use.

\section{Intention to perform less risky sex acts with a new partner}

Perceived behavioral control (53.8\%) and subjective norm (3.4\%) were the strongest predictors of intention to perform less risky sex acts. Here too, intention was low. If scores of participants who strongly intended to perform less risky sex acts were combined (points 6 and 7 of the 7-point Likert-scale measuring intention), only a total of $22.3 \%$ of participants intended to refrain from risky sex acts with a new partner. One possible reason for this could be a lack of knowledge about the per-act risk associated with various sex acts. Most safer-sex programs focus on promoting condom use without emphasizing the risks of certain sex acts (e.g., penetrative anal sex, receptive oral sex). Furthermore, sometimes aberrant definitions of sexual intercourse (as reported by Mehta et al. in 2011) can lead to the performance of high-risk sex acts without identifying these as such, and hence without feeling a need to restrain from them. However, engaging in risky sex practices despite their possible health threats is in accordance with dual-process theory (e.g., Somerville et al., 2010; Steinberg, 2008, 2010) which states that immediate rewardseeking behavior goes hand-in-hand with higher risk-taking and thus with the possibility of risky sexual intercourse. Interestingly, the percentage of participants intending to perform less risky sex acts is comparable to the percentage of participants willing to ask for a new partner's health status. Apparently, refraining from risky sex acts and asking about a new partner's health status were not considered to be rewarding safer-sex practices. It is possible that immediate reward seeking in aroused conditions - and in the absence of a matured self-regulatory capacity - could account for not performing these behaviors, as proposed by dual-process theory. Therefore, immediate pleasure gained from riskier 
sex acts constitutes a considerable risk in terms of acquiring an STI. Consequently, the per-act risk associated with risky sex acts should be emphasized in safer-sex programs in order to enhance the intention to perform less risky sex acts with a new partner.

\section{Intention to use condoms with a new partner}

Perceived behavioral control had the greatest influence on intention to use condoms (this variable predicted $78.3 \%$ of variance), while subjective norms accounted for a much smaller $1.7 \%$ of variance. When measures of participants who strongly intended to use condoms when they next have sexual intercourse were combined (points 6 and 7 of the 7 -point Likert-scale measuring intention, with 7 indicating "completely agree"), a total of $69.7 \%$ of participants intended to use condoms. The number of instances of unprotected sexual intercourse during the last twelve months added $1.1 \%$ predictive power and was identified as a moderating variable of perceived behavioral control related to intention to use condoms. Low perceived behavioral control towards condom use resulted in only moderate intentions to use condoms with a new sex partner, particularly if the number of instances of previous unprotected sexual intercourse was high. In their meta-analysis, McEachan, Conner, Raylor, and Lawton (2011) showed that if RAA variables were controlled for, past behavior still had a strong effect on intentions. The moderating impact of past behavior on future behavior could be explained by various automatic processes such as those proposed by Strack and Deutsch's (2004) Reflective Impulse Model, which also takes habit formation into account. Consistent non-use of condoms in the past, hand-in-hand with low perceived behavioral control related to condom use could, in fact, lead to the non-use of condoms becoming a habit. This is further underscored by the predictive power of reasons for using barrier protection during first sexual intercourse on condom use intention. Even if this variable only predicted $0.4 \%$ of variance, it shows that reasons for past condom use behavior influence current condom use intention. Such combinations of RAA and distal variables should be taken into account when designing behavior change interventions that focus on strengthening perceived control while at the same time mimicking the effects of successful previous condom use.

In alignment with earlier studies (e.g., Casey, Timmermann, Allen, Krahn, \& Turkiewicz, 2009; Gabler, Kropp, Silvera, \& Lavack, 2004; Muñoz-Silva, Sánchez-García, Nunes, \& Martins, 2007; Wulfert \& Wan, 1993), the results indicate the importance of strengthening perceived behavioral control related to using condoms in sexual education programs in order to increase intention to actually use condoms when next having sexual intercourse with a new partner. However, perceived behavioral control regarding condom use is a multidimensional construct (Baele, Dusseldorp, \& Maes, 2001), and it has been shown that intended and performed adolescent condom use are still inconsistent (Sheeran, 2002a). Increasing our understanding of these intricate interplays between RAA and distal variables could, therefore, allow for better tailoring of interventions towards an adolescent population. For instance, understanding how past condom nonuse 
as a distal variable moderates the RAA variable perceived behavioral control, and in this way regulates future action, could help in the design of interventions that simulate successful past condom use behavior among nonusers in order to eventually increase perceived behavioral control related to condom use.

Intentions to perform three safer-sex behaviors (performing less risky sex acts, asking a new sex partner about his/her health status, and using condoms) were mainly predicted by RAA variables, namely perceived behavioral control and subjective norms. High intention related to condom use was sharply contrasted by a low intention related to asking a new sex partner about current health issues and by a low intention to refrain from sexual practices that have been identified as possibly harmful. Including additional distal variables in safer-sex programs may be one helpful way to further promote health-sustaining sexual behavior. Variables such as the level of risk-seeking behavior (concerning the intention to refrain from risky sex acts), or parenting style and unprotected sexual activity (concerning the intention to ask for the current health status) showed significant univariate associations with those intentions. The amount of unprotected sexual intercourse during the last twelve months did not only add predictive power to the intention to use condoms, but it also moderated the impact of perceived behavioral control on intended condom use. Nevertheless, more research is needed to better understand the relationship between distal variables acting as predictors and/or moderators of a specific intention.

Unfortunately, due to a technical problem during data collection, data regarding attitudes concerning the three behaviors of interest could not be collected. Attitudes are important predictors of intention to perform a behavior. However, subjective norms and perceived behavioral control have also been identified as influential predictors and, as such, should be taken into account when designing safer-sex interventions (Fishbein \& Ajzen, 2010). In line with Bandura (1986), who emphasized the predictive utility of selfefficacy beliefs on intentions, self-efficacy - a concept closely related to perceived behavioral control (Fishbein \& Ajzen, 2010) - in particular, has been found to improve the predictive power of intentions - more so than attitude and social norms (Ajzen \& Driver, 1991). This has been confirmed by several studies that have investigated condom use behavior (e.g., Guo et al., 2014; Janepanish, Dancy, \& Park, 2011; Prabawanti, Dijkstra, Riono, \& Hartana, 2014). In summary, although the interpretation of our data remains hampered by the loss of attitude measurements, our results still illustrate the relative importance of perceived behavioral control related to condom use behavior in conjunction with social norms and several distal variables. 


\section{Chapter Two \\ "Don't take my fun away!":}

Expectations of reduced hedonic experience and embarrassment impede implementation of additional safer-sex behavior

Based on:

Brüll, P., Ruiter, R. A. C., Wiers, R. W., \& Kok, G. (under review). "Don't take my fun away!": Expectations of reduced hedonic experience and embarrassment impede implementation of additional safer-sex behavior. 


\section{Abstract}

Condom use during first intercourse with a new sex partner is not performed consistently by young adults, and additional protective measures are not well-known. In this qualitative study, we investigated what factors promote or prevent young adults (18-24 years) to perform additional protective measures. Distal variables were combined with questions assessing salient beliefs about behavioral outcomes, normative referents, control factors, and intentions concerning to (1) perform less risky sex acts, (2) ask a sex partner about his/her sexual health status, and (3) use condoms during next first time sexual intercourse with a new partner. Respondents ( $n=85$ ) mostly perceived condom use as gratification reducing but tolerable while performing less risky sex acts was repeatedly associated with boredom and often rejected on the grounds of reduced hedonic experience. Asking a new sex partner about his/her sexual health status was perceived as embarrassing and unpleasant, leading to widespread rejection within subgroups reporting low intentions to perform all three protective behaviors. Parents and health professionals were reported to exert a normative influence on performing less risky sex acts. Carefully integrating additional safer-sex measures into safer-sex promoting programs could increase awareness of risky sexual behavior that cannot be eliminated by condom use alone. 


\section{Introduction}

World-wide, young adults aged 15-24 years acquire nearly half of all new curable sexually transmitted infections (STIS) per year, accounting for approximatively a quarter of a billion new cases (World Health Organization, 2013). Some factors make young adults particularly vulnerable to STIs. For example, research has shown that young adults often show variations in their definitions of sexual acts, which may impact their ability to assess risk (Mehta et al., 2011). Moreover, young adult sexual relationships tend to be brief (Henderson et al., 2002). Finally, young adults are still in a developmental period characterized by changes in self-regulatory capacity and pronounced reward-seeking behavior (Casey et al., 2011; Somerville et al., 2010; Steinberg, 2008, 2010), alternating with periods of boredom and "low levels of momentary positive affect" (Forbes \& Dahl, 2012). Recent data released by the World Health Organization for 2011 show an increase of curable but (if left untreated) harmful STIs, with Chlamydia Trachomatis, Neisseria Gonorrhoeae, and Trichomonas Vaginalis the most prevalent in individuals between 15 and 49 years of age (World Health Organization, 2012). Worldwide, an estimated 35.3 million people were known to be infected with human immunodeficiency virus (HIV) in 2012 with 900,000 people infected in Western and Central Europe.

Reduced access to quality STI prevention services (due to lack of health insurance, lack of monetary resources, and discomfort with facilities) makes young adults more prone to acquiring STIs than adults (Centers for Disease Control, 2012). Worldwide, only about $41 \%$ of young adults reported using a condom the last time they had sex with a new partner (World Health Organization, 2014). Reframed, this means that $59 \%$ of young adults do not consistently use condoms with a new partner in order to protect themselves or their partners. In addition, young adults have a tendency to underestimate their risk for STIS (O'Sullivan et al., 2010; Wolfers et al., 2011). Following infection, the risk of HIV transmission remains substantial, even during asymptotic stages (Hollingsworth et al., 2008; Kim \& Koopman, 2012). It is, therefore, crucial to better understand the reasons why young adults might refrain from using barrier protection.

Normative factors, availability, and costs have all been identified as intrinsic factors related to male condom use (Nahmias \& Nahmias, 2011), suggesting that additional health-sustaining sexual behavior might reduce the risk of HIV infection (and of STI infection in general). As reported by Varghese et al. (2002), abstinence from insertive anal sex in favor of insertive fellatio reduces the relative risk of HIV infection 13-fold, while using condoms and choosing a sex partner who tested negative (as compared to an untested sex partner) reduces the relative risk of HIV infection 20-fold and 47-fold, respectively. Moreover, recent data indicate an even more pronounced risk of HIV acquisition for receptive anal intercourse than previously estimated (P. Patel et al., 2014a). A better understanding of factors influencing young adults' decision to adopt these three safer-sex behaviors could help improve current and future safer-sex education programs. 
The Reasoned Action Approach (RAA) (Fishbein \& Ajzen, 2010) is an accurate explanatory and predictive model that can be used to identify determinants of intention to perform specific safe-sex behaviors. This model emerged from the Theory of Reasoned Action (TRA) (Fishbein \& Ajzen, 1975) and the Theory of Planned Behavior (TPB) (Ajzen, $1985,1991)$. These social cognition models of human behavior suggest that behavioral decisions are made in the light of reasoned considerations derived from available information about the costs and benefits of the behavior, the extent to which the behavior is socially acceptable, and to what extend executing the behavior is believed to be under personal control. It has been observed that young adults engage in more risky behaviors than adults and for this reason, it has been inferred that they lack optimal reasoning abilities (Arnett, 1992; Spear, 2009). However, under optimal conditions, young adults are perfectly able to make rational decisions in order to achieve their goals (Gardner \& Steinberg, 2005). Moreover, it has been strongly argued that young adults do not have flawed reasoning capabilities or poor decision-making skills per se (Reyna \& Farley, 2006). Only in the heat of the moment, in unfamiliar situations, in the presence of peers and when behavioral inhibition is required to achieve a good outcome, do young adults tend to reason more poorly than adults. Under these conditions, they are indeed more likely to engage in health-compromising behaviors (Reyna \& Farley, 2006). In line with this research, it has been shown that safe sexual intercourse, especially condom use, is implemented inconsistently at best by the majority of sexually active young adults (Finer \& Philbin, 2013; Tschann et al., 2010).

Numerous studies have investigated risk factors associated with young adult sexuality, namely substance use (Cornelius, Clark, Reynolds, Kirisci, \& Tarter, 2007; Madkour, Farhat, Halpern, Godeau, \& Gabhainn, 2010), socioeconomic status (Cubbin et al., 2010; Morrison-Beedy et al., 2012; Van Ryzin et al., 2011), family factors (Hipwell, Stepp, Keenan, Chung, \& Loeber, 2011; Ladapo et al., 2013; Morales-Campos, Markham, Peskin, \& Fernandez, 2012; Morrison-Beedy et al., 2012), peer and school contexts (Coley et al., 2013; Lansford et al., 2010; Markham et al., 2010; Young \& Vazsonyi, 2011), cognitive and personality factors (Crockett et al., 2006; House et al., 2010; Khurana et al., 2012; Schofield et al., 2008), and religiosity (Grossman et al., 2013; Landor et al., 2011). However, surprisingly little is known about young adults' self-reported explicit attitudes regarding safe sexual acts (Halpern-Felsher, 2008; Halpern-Felsher, Cornell, Kropp, \& Tschann, 2005). So far, studies addressing this issue have collected mainly quantitative data (Teva, Bermudez, Ramiro, \& Ramiro-Sanchez, 2013). Some qualitative studies have assessed factors influencing sexual risk-taking among young heterosexual adults (Corneille, Tademy, Reid, Belgrave, \& Nasim, 2008; Dietrich et al., 2011; Morojele, Brook, \& Kachieng'a, 2006) and among young adult men who have sex with men (Horvath, Rosser, \& Remafedi, 2008). Surprisingly, information about explicit reasons for young adults to engage in risky sexual behavior, such as unprotected insertive anal sex, remains scarce. Factors found to negatively influence safer-sex practices include: The type of sexual relationship (Bauman \& Berman, 2005), consent to unwanted sexual activity (sexual 
compliance) with the aim of satisfying a partner's needs or promoting intimacy (O'Sullivan \& Allgeier, 1998; Vannier \& O'Sullivan, 2010), contextual barriers such as coercion or manipulative behavior by a new sex partner due to gendered power differences (Melendez et al., 2003; Taylor, 1995), compromised pleasure (Bauman, Karasz, \& Hamilton, 2007), and highly interactive forms of modern communication technologies such as texting (O’Sullivan, 2015).

The current study was designed to allow young adults, aged 18-24 years, to express their attitudes and perceptions about safer-sex measures, anonymously and without being limited by predefined answers, in an online questionnaire consisting of open-ended questions. In total, 85 young adults answered open questions about the reasons behind why they do or do not (1) engage in less risky sex acts, (2) ask a new sex partner about his/her sexual health status, and (3) use condoms during sexual intercourse. Risky sex acts were defined as unprotected insertive anal sex or rough sexual practices that could evoke minor vaginal lesions. Combining variables derived from the Reasoned Action Approach with questions about participants past protective behavior, STI and HIV testing history, and current estimation of sexual health, we investigated which factors encourage young adults to carry out these three protective behaviors or prevent them from doing so.

\section{Materials and methods}

\section{Participants and procedure}

The present study was part of a larger survey on explicit and implicit factors associated with young adult decision making processes concerning safer-sex behavior. This larger study used both quantitative and qualitative methods. In this paper, we report the qualitative findings. The study was conducted using a German online panel (www.meineumfrage.com) that specialized in academic and social research. Our sample was socially, culturally and socio-economically diverse. All participants were aged between 18 and 24 years, were sexually active, heterosexual, and not in a relationship at the time of the survey (for those in a relationship, STI reducing safer-sex precautions play a diminished role in comparison to increased contraceptive measures (Bauman \& Berman, 2005; Manlove, Ryan, \& Franzetta, 2004; Manlove \& Terry-Humen, 2007)). After providing their consent, participants were linked to a secured website that contained the study materials. The introductory page explained that the study was looking for possible connections between conscious and unconscious factors involved in the process of decision making related to safer-sex behavior. Participants were rewarded with panel credits which they could accumulate along with credits received from participation in previous studies and ultimately exchange for money. Moreover, if they wished, participants could enter a lottery in order to win one of three online shopping credit vouchers (€50,- each). Anonymity was assured as e-mail addresses were not linked to individual data. Completing the questionnaire took approximately 25 to 30 minutes. 
Out of 1114 questionnaires that were accessed online, 242 were completed and returned $(21.7 \%)$. This high drop-out rate is not unusual for this particular panel because participants are encouraged to visit the sites containing study materials and only then to decide whether to participate or not. Of course, visiting the website out of curiosity is counted as access but is only rewarded with panel credits after completion of the survey. One hundred and fifty-seven participants were excluded because they were either older than 24 years or did not answer the questions related to the qualitative part of the survey (reported in this paper). This left a sample of 85 sexually experienced heterosexual participants for analysis. The majority of the respondents were male (5 $3 \%, n=45$ ), with a mean age of 23.6 years. The main age of the female participants was $2 \quad 2.7$ years $\left(\begin{array}{llll}4 & 7 \% & 0\end{array}\right.$

\section{Measures}

Participants' gender, age, protection against STIs during their first sexual intercourse (none, barrier protection, dual protection, not sure), and their own assessment of currently having an (undiagnosed) STI were all assessed using close-ended questions with appropriate answer categories. Participants were also asked if they had ever taken an HIV test and if they had taken an STI test during the last 12 months. Behavioral intention, defined as the readiness to engage in a specific behavior and hence determining the actual behavior (Fishbein \& Ajzen, 2010), was assessed separately by the items "I intend to perform less risky sex acts / ask a new sex partner about his/her sexual health status / use condoms when I have sex with a new sex partner for the first time", and measured using a 7-point Likert-scale ranging from (1) "unlikely" to (7) "likely". To provide clarity, "risky sex practices" were defined as unprotected insertive anal sex or rough sexual practices that could evoke minor vaginal or penile lesions.

Open-ended questions based on the Reasoned Action Approach (Fishbein \& Ajzen, 2010) assessed salient beliefs about behavioral outcomes, normative referents, and control factors concerning (1) engaging in less risky sex acts, (2) asking a sex partner about his/her sexual health status, and (3) using condoms during next sexual intercourse.

Salient behavioral beliefs. Salient behavioral beliefs represent the information individuals have about a specific behavior. This information then serves as the foundation for attitudes towards this behavior (Fishbein \& Ajzen, 2010). These beliefs were assessed by open questions, such as "What do you identify as the advantages of engaging in less risky sex acts when you have sex for the first time with your next sex partner?"

Control factors. Control factors can be internal to an individual (for example, skills and willpower), or external, (for example, task demands and the actions of other people) (Fishbein \& Ajzen, 2010). As the three (sexual health-related) protective behaviors of interest are likely to be influenced by both factors, we were interested in participants' general expectations regarding controllability of these behaviors. To this end, control factors were assessed by questions such as: "Please list any factors or circumstances that you 
can think of which would make it easy for you (or enable you) to use condoms with your next sex partner when you have sex for the first time with him/her."

Normative referents. Injunctive norms related to a specific behavior can be described as an individual's beliefs about what other people, whose opinions about a specific behavior are important to this individual, view as appropriate behavior. These people are defined as normative referents (Fishbein \& Ajzen, 2010). Individuals or groups acting as normative referents were identified by open questions following a general introduction, for example "When it comes to using condoms with your next sex partner (when you have sex for the first time with him/her), there might be individuals or groups who think you should or should not do this." Normative referents were then assessed by questions, such as: "Please list the individuals or groups who would approve of you using condoms - or think you should use condoms - with your next sex partner (when you have sex for the first time with him/her)."

\section{Data processing and analysis}

Data were managed with the qualitative data management software NVivo version 10 (QSR, Doncaster, Australia) and analyzed by the first author, who conducted a thematic analysis (Braun \& Clarke, 2006) to establish and explore themes. A sample of $30 \%$ of the data was coded by an independent researcher. The initial coding was then compared, reviewed, and finally refined in order to achieve a more representative coding. After consensus was reached on how the data were coded, the codebook was updated, and the remaining data were coded by the first author. Results are structured according to the themes that emerged from this analysis and quotations representing themes are included to demonstrate study findings. These quotations were translated from German to English by the first author - who is native German-speaking - and then back-translated to German by an independent researcher (also a native German speaker) to ensure the accuracy of the English translation.

\section{Results}

Risky sex acts

Salient beliefs related to engaging in less risky sex acts

Reduced sexual pleasure. Most respondents reported associations with boredom and reduced sexual gratification. A 22-year-old female mentioned: "In my opinion, always living on the safe side of life makes you lose something. It is simply less fun! How does the 'thrill' remain if you always do the same things in bed?" Some respondents further stated that 
boredom and reduced sexual gratification are disadvantages of engaging in less risky sex acts. One 24-year-old male stated: "Enjoying sex would surely be hampered."

Protection against STIs. Most respondents associated less risky sex acts with protection. A 20-year-old female reported: "You don't get diseases! That's for sure!" Some respondents reported that reducing the risk of acquiring an STI would be an advantage of engaging in less risky sex acts. One 24-year-old female answered: "I can be relaxed afterwards, also long-term because I can be certain about my health status. I can also be self-confident because I stood up for myself."

Factors which facilitate or complicate engaging in less risky sex acts

Most respondents felt that an understanding sex partner would be open to engaging in less risky sex acts. A partner who prefers/could be trusted to engage in less risky sex acts was seen as a facilitating factor. Some respondents described a sex partner opposed to engaging in less risky sex acts as a potentially complicating factor, and some added that alcohol intake or other drug consumption could complicate the implementation of less risky sex acts. Only a few respondents mentioned strong sexual arousal as a complicating factor. A 24-year-old male who defined "normal sex" as insertive fellatio stated: "It would be very difficult if there is an intense desire to experience this rush, knowing that you would miss these intense sensations while having just normal sex."

Normative referents related to engaging in less risky sex acts

Who is expected (not) to engage in less risky sex acts? Most respondents expected that individuals with reduced behavioral control (due to alcohol intake or other drug consumption) would not refrain from engaging in risky sex acts. Friends were identified by some respondents as individuals expected to engage in less risky sex acts. More generally, respondents thought that "boring" individuals would refrain from engaging in risky sex acts, as illustrated by one 23-year-old female who reported that "the more boring kind of people, like academics" would engage in less risky sex acts.

Who approves of (not) engaging in less risky sex acts? Most respondents reported that their parents or family, as well as their friends, would approve of their engagement in less risky sex acts. Some stated that health professionals, in general, were approving of less risky sex acts and that no one would disapprove of their engagement in less risky sex acts. Only a few respondents expected that their friends or their sex partner would disapprove of them engaging in less risky sex acts. One 24-year-old male stated: "Virtually no sex partner likes such reservations, they all look forward to having sex." 
Asking about sexual health status

Salient beliefs related to asking a new sex partner about his/her sexual health status

Embarrassment. Most respondents reported having unpleasant associations with asking a sex partner about previous or current STIs. These respondents mentioned that asking their sex partner about his/her sexual health status would most certainly "kill the atmosphere" and terminate a probable sexual encounter. A 22-year-old female stated:" How embarrassing! And you don't know if the answer is correct." Some respondents described the unpleasantness related to asking such a question as disadvantageous, and they associated asking about a partner's sexual health status with embarrassment. However, only a few respondents indicated that their embarrassment constitutes a disadvantage of asking this question.

Fear of rejection. Most respondents associated asking a new sex partner about his/her sexual health status with fear of being rejected. A 19-year-old female stated: "Maybe he doesn't want to have sex anymore or I don't want to have sex with him anymore." Some respondents specified that asking such a question could express a lack of trust in their partner - resulting in possible rejection - and would, therefore, constitute a disadvantage.

Protection against STIs. Most respondents agreed that asking a sex partner about his/her sexual health status would at least reduce the risk of acquiring an STI. However, relatively few respondents identified this behavior as normal or even as necessary in order to protect their health. One who did, a 24-year-old girl, described it as a "legitimate question" to ask.

Trust. Most respondents also reported that asking this question could increase trust in a new sex partner. Those partners who were not offended by the question were generally seen as more trustworthy than those who reacted defensively. A 20-year-old female reported that she would be more likely to trust someone who is not offended by this question because his "reaction shows how tolerant he is." Another 20-year-old female mentioned that "this level of honesty should be a precondition" to engaging in sexual intercourse.

Factors facilitating or complicating asking a new sex partner about his/her sexual health status

Most respondents reported that a new sex partner who seems open to discussing his/her sexual health status would facilitate the asking of this question. They stated that the likelihood of establishing such a level of openness and trust would be increased if they were "already befriended for some time," as one 22-year-old female put it. Other respondents mentioned that not knowing their new sex partner well would be a complicating factor. A 24-year-old girl stated that she would feel "insecure" and "fear his reaction" if she did not know her potential sex partner well. Some respondents identified reduced behavioral control (due to alcohol intake or other drug consumption) as a potentially complicating factor. This is illustrated by one 22-year-old male who reported that, in all likelihood, he 
would refrain from answering the question if he "had been drinking quite a lot." Relatively few respondents reported that strong sexual arousal would complicate asking a new sex partner about his/her sexual health status.

Normative referents related to asking a new sex partner about his/her sexual health status

Who is expected (not) to ask his/her new sex partner about his/her sexual health status? Most respondents expected that their friends would not ask their new sex partners about their sexual health status, as illustrated by one 24-year-old male who stated: "Most of my friends would not do this. " However, most respondents expected "reasonable individuals" as put by one 24-year-old female, to ask potential sex partners about their sexual health. Some participants mentioned that health professionals would ask their new sex partner before having first-time sex. However, they did not expect that individuals with reduced behavioral control (due to alcohol intake or other drug consumption), or as one 24-yearold male put it, "all those intoxicated individuals" would ask their sex partner about their sexual health status. Only a few respondents mentioned that individuals with a "low sense of responsibility towards their own health" as put by a 23-year-old female, would refrain from asking this question.

\section{Who approves of (not) asking his/her new sex partner about his/her sexual health status?} Most respondents reported that their parents or family would approve of them asking their new sex partners about their sexual health status. A 20-year-old male wrote: "My parents would approve, for sure! However, it would be slightly awkward to talk to them about it." Furthermore, most respondents thought that their friends would approve of them asking their sex partners, but that their new sex partner would likely disapprove of them asking. One 22-year-old female reported: "Oh, my new sex partner would not like it. I'm sure! How embarrassing!" Some respondents mentioned that health professionals, in general, would approve of them asking and that no one would disapprove of them asking their new sex partner about his/her sexual health status.

\section{Condom use}

Salient beliefs related to condom use

Protection against STIs. Most respondents associated condom use with protection against STIs, and identified condom use as advantageous in terms of protecting themselves against STIs or preventing an unwanted pregnancy. For example, one 23-year-old male stated: "This is the best protection against venereal diseases.", and one 20-year-old female reported that using condoms "clearly reduces the risk of becoming pregnant or contracting a social disease." 
Reduced sexual pleasure. Besides protection against STIs, most respondents identified a reduced hedonic experience, namely a condom-induced reduction of sensation and sexual gratification, as a disadvantage of condom use. A 24-year-old male stated: "Having sex using a condom just feels different. You can't compare it! I definitively prefer having sex without condoms."

Factors facilitating or complicating condom use

Most respondents mentioned that a sex partner "who opposes condom use," as put by one 22-year-old female, complicates implementation. Most respondents also mentioned not having condoms readily available as a further complicating factor. As a 24-year-old female stated: "If you first have to find a condom machine or need to go to a store to buy some, well, that would just kill sexual excitation." Some respondents mentioned the same factors in relation to how they could facilitate condom use. For example, a sex partner who agrees to use condoms and have condoms readily available. As one 24-year-old female stated: "You need to have them with you all the time, and if you don't, then you go out to get them and can enjoy sex afterwards."

Normative referents related to condom use

Who is expected (not) to use condoms? Most respondents reported that their friends would be likely to use condoms during first time sex with a new sex partner. They further reported that they did not expect individuals with reduced behavioral control (due to alcohol intake or other drug consumption) to use condoms. Some respondents expected that members of their family would use condoms during new sexual encounters. Only a few respondents mentioned that individuals with a low sense of responsibility would not be likely to use condoms.

Who approves of (not) using condoms? Most respondents reported that their friends would approve of them using condoms during first time sex with a new partner, and that their parents or family would approve of their use of condoms. Some respondents expected that some of their friends would disapprove of them using condoms, and that a new sex partner would also disapprove of condom use. In fact, only a few respondents mentioned that a new sex partner would be very likely to approve of condom use. Table 1 shows an overview of these results. 
Table 1. Overview of factors influencing specific safer-sex behavior

\begin{tabular}{|c|c|c|c|}
\hline & Less Risky Sex Acts & Asking Sexual Health Status & Condom Use \\
\hline \multicolumn{4}{|l|}{ Salient beliefs } \\
\hline & $\begin{array}{l}\text { - reduced hedonic experience } \\
\text { - protection against STIs }\end{array}$ & $\begin{array}{l}\text { - unpleasantness } \\
\text { - embarrassment } \\
\text { - possible rejection } \\
\text { - protection against STIs }\end{array}$ & $\begin{array}{l}\text { - protection against STIs } \\
\text { - reduced hedonic experience } \\
\text { - unreliable availability }\end{array}$ \\
\hline \multicolumn{4}{|c|}{ Normative referents } \\
\hline Who does & $\begin{array}{l}\text { - boring individuals } \\
\text { - cautious individuals }\end{array}$ & - health professionals & $\begin{array}{l}\text { - friends } \\
\text { - family }\end{array}$ \\
\hline Who does not & - drunk/drugged individuals & $\begin{array}{l}\text { - drunk/drugged individuals } \\
\text { - irresponsible individuals }\end{array}$ & $\begin{array}{l}\text { - drunk/drugged individuals } \\
\text { - irresponsible individuals }\end{array}$ \\
\hline Who approves & $\begin{array}{l}\text { - parents/family } \\
\text { - health professionals }\end{array}$ & $\begin{array}{l}\text { - parents/family } \\
\text { - friends } \\
\text { - health professionals }\end{array}$ & $\begin{array}{l}\text { - friends } \\
\text { - parents/family } \\
\text { - new sex partner }\end{array}$ \\
\hline Who disapproves & - new sex partner & - new sex partner & $\begin{array}{l}\text { - friends } \\
\text { - new sex partner }\end{array}$ \\
\hline \multicolumn{4}{|l|}{ Control factors } \\
\hline Facilitating & $\begin{array}{l}\text { - supporting new sex partner } \\
\text { - trust in sex partner }\end{array}$ & $\begin{array}{l}\text { - supporting new sex partner } \\
\text { - trust in sex partner }\end{array}$ & $\begin{array}{l}\text { - supporting new sex partner } \\
\text { - having condoms at hand }\end{array}$ \\
\hline Complicating & $\begin{array}{l}\text { - opposed new sex partner } \\
\text { - being drunk/drugged } \\
\text { - instant sexual gratification }\end{array}$ & $\begin{array}{l}\text { - unfamiliar new sex partner } \\
\text { - being drunk/drugged } \\
\text { - instant sexual gratification }\end{array}$ & $\begin{array}{l}\text { - opposed new sex partner } \\
\text { - not having condoms }\end{array}$ \\
\hline
\end{tabular}

Respondents with high versus low intention to implement all three behaviors

Out of the 85 participants, thirty respondents ( 9 male, 21 female) indicated a high intention (5 to 7 on a 7-point Likert-Scale with the highest value representing "likely") to implement all three protective behaviors the next time s/he would have sexual intercourse (for the first time) with a new sex partner. Eighteen respondents had been tested for HIV and eight of these individuals had additionally been tested for other STIs. All reported that they were sure they did not have any STIs apart from one respondent who indicated that it was possible that $\mathrm{s} /$ he had acquired an STI.

Fourteen respondents (12 male, 2 female) indicated a low intention ( 1 to 3 on a 7point Likert-Scale with the highest value representing "likely") to implement all three protective behaviors the next time s/he would have sexual intercourse (for the first time) with a new sex partner. No respondent from these fourteen individuals had been tested for STIS (including HIV) so far, but all reported they were sure they did not have any STIS.

Gender difference. The only gender difference we identified was related to the intention to perform all three safer-sex behaviors. More females than males indicated a high intention to implement all three protective behaviors the next time s/he would have sexual intercourse (for the first time) with a new sex partner.

Performing less risky sex acts. For most respondents with high intentions to implement all three safer-sex behaviors, health protection was identified as the main advantage of 
engaging in less risky sex acts, and reduced sexual gratification and "less fun" were identified as the main disadvantages. Family and health professionals were identified as people who would approve of less risky sex acts, and no-one was identified as being disapproving. Some respondents mentioned that only self-dependent individuals were supposed to perform less risky sex acts - as opposed to individuals with a low sense of responsibility who were thought to refrain from this kind of protective behavior. Additionally, a new sex partner who is appreciative of performing less risky sex acts was identified as being a facilitating factor. No complicating factors were mentioned.

Most respondents with low intention associated engaging in less risky sex acts with boredom and reduced hedonic experience. Boredom was further mentioned as disadvantageous in terms of implementing this behavior although respondents also indicated health preservation as an advantage of implementation. Some respondents reported that their families would likely approve of their restraint from risky sex acts, but they thought that a new sex partner would disapprove of this protective behavior. Furthermore, some respondents stated that only boring or conformist individuals would refrain from risky sex practices and that individuals "who want to have good sex" would not refrain. They did not identify any facilitating factors but expressed their uncertainty about a sex partner's reaction to this behavior being a complicating factor in its implementation.

Asking a new sex partner about his/her sexual health status. Most respondents high in intention associated asking a new sex partner about his/her sexual health status with embarrassment and awkwardness. These respondents identified health protection as an advantage but nevertheless reported that they would feel uncomfortable asking a new sex partner about his/her sexual health. Moreover, some mentioned that such a question could easily disrupt an intimate atmosphere. Parents and family, as well as health professionals, were identified by some respondents as individuals who would approve of them asking their sex partner. No examples were provided of individuals who would disapprove of this. Some respondents stated that health professionals would likely perform this behavior but that only a few friends would do so. Individuals with reduced intellectual abilities were also reported as being unlikely to ask their new sex partners about their sexual health status. Confidence was identified as a facilitating factor. Only a few respondents identified increased sexual desire as a complicating factor.

For most respondents with low intentions, asking a new sex partner about his/her sexual health status was associated with mistrust and awkwardness. These respondents did identify a possible health sustaining advantage to asking but disadvantages centered around insecurity about their new sex partner's reaction and the likely obliteration of a sexual intercourse-sustaining atmosphere. Some respondents identified parents and siblings as individuals who would approve of asking, and a new sex partner as someone likely to disapprove of them implementing this behavior. No respondent could name a normative referent who would or would not ask a new sex partner about his/her sexual health 
status. Finally, they did not identify any facilitating factors but mentioned that "everything" would complicate implementation.

Using condoms. For most respondents high in intention, condom use was associated with protection against STIs, and this was identified as the main advantage of using condoms. A reduction of skin sensibility was most frequently mentioned as a disadvantage of condom use. The respondents could not identify anyone who would disapprove of condom use, was identified and parents, family, friends, and health professionals were most often mentioned as individuals who would approve. Some respondents identified friends - and responsible individuals in general - as likely to use condoms, as opposed to irresponsible individuals who were reported as being reluctant to use condoms. Furthermore, some respondents identified the availability of condoms at the very moment of a possible sexual encounter as a facilitating factor and the absence of condoms as a potentially complicating factor. Only a few respondents mentioned a disapproving new sex partner as being a potentially complicating factor for condom use.

All respondents low in intentions associated condom use with protection against STIS and identified this as the main advantage of using condoms. Most respondents associated condom use with reduced sexual pleasure and reported this as a disadvantage. Furthermore, most respondents also identified parents as individuals who would approve of their condom use whereas friends and possible new sex partners were identified as individuals who would disapprove. Some respondents described individuals using condoms as being boring. However, they were unable to identify individuals or groups who would refrain from condom use. For some respondents, no facilitating factors exist, but reduced hedonic experience was identified as disadvantageous. Table 2 shows an overview of these results. 
Table 2. Overview of factors influencing specific safer-sex behavior by intention

\begin{tabular}{llll}
\hline \multicolumn{2}{c}{ Less Risky Sex Acts } & Asking Sexual Health Status & Condom Use \\
\hline Low Intention ( $\mathbf{N}=\mathbf{1 ~ 4}$ ) & & \\
Salient beliefs & & \\
& - reduced hedonic experi- & - invoked mistrust & - protection against STIs \\
& ence & - awfulness & - reduced hedonic experi- \\
& - boredom & - protection against STIs & ence \\
& & - unknown reaction of sex & \\
& partner
\end{tabular}

\section{Normative referents}

\begin{tabular}{|c|c|c|c|}
\hline \multirow{2}{*}{\multicolumn{4}{|c|}{$\begin{array}{l}\text { Who does } \\
\text { Who does not }\end{array}$}} \\
\hline & & & \\
\hline Who approves & - parents/family & - parents/family & - parents/family \\
\hline Who disapproves & - new sex partner & - new sex partner & $\begin{array}{l}\text { - new sex partner } \\
\text { - friends }\end{array}$ \\
\hline
\end{tabular}

\section{Control factors}

Facilitating

Complicating
- unknown reaction of part- - "everything" ner
- reduced hedonic experi- ence

High Intention ( $=30$ )

\section{Salient beliefs}

$\begin{array}{lll}\text { - reduced hedonic experi- } & \text { - unpleasantness } & \text { - protection against STIs } \\ \text { ence } & \text { - embarrassment } & \text { - reduced hedonic experi- } \\ \text { - protection against STIS } & \text { - disrupted atmosphere } & \text { ence } \\ & \text { - protection against STIS } & \end{array}$

\section{Normative referents}

\begin{tabular}{|c|c|c|c|}
\hline Who does & - self-dependent individuals & - health professionals & $\begin{array}{l}\text { - friends } \\
\text { - responsible individuals }\end{array}$ \\
\hline Who does not & - irresponsible individuals & - ignorant individuals & - irresponsible individuals \\
\hline Who approves & $\begin{array}{l}\text { - parents/family } \\
\text { - health professionals }\end{array}$ & $\begin{array}{l}\text { - parents/family } \\
\text { - health professionals }\end{array}$ & $\begin{array}{l}\text { - friends } \\
\text { - parents/family } \\
\text { - health professionals }\end{array}$ \\
\hline \multicolumn{4}{|c|}{ Who disapproves } \\
\hline \multicolumn{4}{|l|}{ Control factors } \\
\hline Facilitating & $\begin{array}{l}\text { - supporting new sex part- } \\
\text { ner }\end{array}$ & - trust in sex partner & - having condoms at hand \\
\hline Complicating & & - instant sexual gratification & $\begin{array}{l}\text { - opposed new sex partner } \\
\text { - not having condoms }\end{array}$ \\
\hline
\end{tabular}

\section{Discussion}

In this study, we describe salient beliefs, control factors, and normative referents related to three different safer-sex behaviors. Furthermore, behavioral intention was identified, and responses were compared across levels of intention to perform all three protective behaviors. Our findings show that all three safer-sex behaviors were believed to provide 
protection against STIs. However, the intention to implement these behaviors during the next (first time) sexual encounter with a new sex partner varied between respondents. Such inconsistencies between knowledge and intentions and their negative effect on translation into behavior have been observed before (Paschal Sheeran, 2002b), particularly in relation to condom use behavior (Bauman et al., 2007; Turchik \& Gidycz, 2012). Our study confirmed this inconsistency in relation to condom use behavior. Moreover, it demonstrates that this inconsistency between knowledge and intentions also exists in relation to asking a new partner about his or her sexual health and refraining from risky sex practices with a new sex partner.

The most commonly cited reason for refraining from less risky sex acts and using condoms was reduced hedonic experience during sexual intercourse, eventually resulting in diminished sexual gratification. This prioritizing of sexual gratification over health protection has been observed previously (Hill \& Durante, 2011a; O'Sullivan et al., 2010). However, in the present study, respondents did not ignore a possible health threat due to unprotected sexual intercourse. However, when low in intention to implement all three behaviors, respondents did not acknowledge the risk of contracting an STI. This observation is in line with Wolfers et al. (2011), who identified an underestimation by young adults of the risk of contracting STIs, and with Wu et al. (2005), who reported an influence of prior sexual risk experience on current sexual behavior. This gap between knowledge and intentions to engage in protective behavior was further enlarged by a reported unpleasantness and embarrassment pertaining to asking a new sex partner about his/her sexual health status. Engaging in risky sex acts was mostly justified in terms of the reduced sexual gratification resulting from "boring" sexual intercourse. Refraining from condom use was mostly explained in terms of an association between condoms and reduced sexual gratification due to a material (mostly latex)-related loss of sensitivity, as also reported by Crosby et al. (2013). The possibility of rejection by a sex partner was cited as the main reason not to ask a new sex partner about his/her sexual health status. This sensitivity to social evaluation displayed by young adults has previously been reported by Somerville (2013), and constitutes a main obstacle for respondents of the present study inquiring about their new sex partner's sexual health status. As compared to respondents high in intention to perform all three protective behaviors, respondents low in intention were more likely to associate protective measures with boredom, mistrust, and uncertainty with regard to a new sex partner's reaction. Furthermore, respondents low in intention to implement all three behaviors identified more disadvantages than advantages related to each safer-sex behavior as compared to respondents high in intention, who had a more balanced view of the advantages and disadvantages.

Previous studies have observed that young adults' decision making is prone to peer influences (Albert et al., 2013; Gardner \& Steinberg, 2005; Shad et al., 2011; Sturman \& Moghaddam, 2012; Weigard, Chein, Albert, Smith, \& Steinberg, 2014). The importance of peers as normative referents - not only for adolescents but also for young adults - has been reported by Steinberg and Monahan (2007), and identified in relation to health- 
threatening behaviors such as cigarette use, binge drinking, and marijuana use (Andrews, Tildesley, Hops, \& Li, 2002), as well as for long-run outcomes such as IQ scores, teenage childbearing, education, and labor market outcomes (Black, Devereux, \& Salvanes, 2013). Most respondents in our study reported an influence of friends on their decisions related to sexual health protection and reported that friends would approve of them asking a new sex partner about his/her sexual health status. In general, respondents described friends as being approving of condom use more frequently that they described friends as being disapproving of condom use. However, it was mainly participants already high in intention to adopt all three safer-sex behaviors who reported that their peers would approve of condom use, while the majority of participants with a low intention to adopt all three safer-sex behaviors indicated that their peers would disapprove of condom use.

Parents and family members were identified as important normative referents who would approve of all three protective behaviors. This finding is in accordance with models of human development that accord caregivers - and especially parents - a direct influence on the child via parenting style and parental behavior, while also allocating a proportion of influence to other family members via shared genetics (Baumrind, 1978, 1991; Beyers \& Goossens, 1999; Bronfenbrenner, 1979). Previous research has demonstrated that parental influence remains a primary protective factor for young adults (Kotchick, Shaffer, Forehand, \& Miller, 2001; Zimmer-Gembeck \& Helfand, 2008). Moreover, it has been demonstrated that, particularly in relation to youth alcohol prevention programs, not only involving parents but also targeting both parents and young adults leads to greater success (Koning, Maric, MacKinnon, \& Vollebergh, 2015). Nevertheless, our study shows that although parents were reported to exert a normative influence on the majority of respondents, not all of these respondents reported intention to adopt all three protective behaviors. In fact, most respondents with high intention to adopt all three protective behaviors as well as most respondents with low intention to do so reported family and parents as normative referents who would approve of them performing these protective behaviors. Whilst young adults are not expected to explicitly talk about their sexual protection behavior with their parents, we learned that, nevertheless, parents still exert a normative influence on their children's safer-sex behavior, even if parents were not expected to engage in these behaviors themselves.

Health professionals, especially physicians, were also identified as individuals who would approve of all three safer sex behaviors, but only by female respondents who reported a high intention to implement all three behaviors. Respondents (both high- and low-intention) identified health professionals as individuals who would approve of them performing less risky sex acts and asking about the current sexual health status of a new partner. However, none of the respondents with low intentions identified health professionals as normative referents. Interestingly, respondents with high intentions also expected health professionals to implement these behaviors, contrary to their expectations towards their own parents' sexual behavior. This underlines the normative power health care professionals have over young adults (Brotonsc et al., 2005), especially for females 
high in intention to perform all three safer-sex behaviors. Furthermore, participants with high intention to perform all three behaviors identified self-dependent and responsible individuals as those most likely to perform less risky sex acts and to ask about the current sexual health status of a partner. On the other hand, most respondents low in intention to perform all three protective behaviors considered individuals engaging in less risky sex acts and asking about the current sexual health status of a partner as "boring" or "overly cautious."

In general, respondents did not expect that individuals who abused alcohol or other drugs would be likely to implement health protective sexual behavior. Alcohol consumption or other drug intake, as well as the desire for instant sexual gratification, were mentioned as potentially complicating factors in relation to performing less risky sex acts and for asking a new sex partner about his/her sexual health status. This points to a particular vulnerability of young adults in certain circumstances (i.e., after alcohol or drug consumption and in combination with increased sexual appetite). As Ariely and Loewenstein (2006) have shown, high levels of sexual arousal increase the willingness to engage in risky sexual activities and sexual activities that are already attractive when not sexually aroused become even more attractive while being sexually aroused. Excessive alcohol or drug consumption combined with increased sexual arousal could, therefore, present an increased risk of contracting STIs. This is in line with Ebel-Lam, MacDonald, Zanna, and Fong (2009) who showed that alcohol intoxication combined with increased sexual arousal led to an increased likelihood of engaging in unprotected sex and further underlines the link between intoxication and risky sexual behavior.

It was often expected that a new sex partner would disapprove of all three protective behaviors, and this was therefore identified as a complicating factor in terms of the implementation of protective sexual health behaviors. Accordingly, a new sex partner who supports all three safer-sex behaviors, as well as a new sex partner who is perceived as trustworthy, were both seen as facilitating factors. This is in line with the findings of Michaelson, de la Vega, Chatham, and Munakata (2013), who demonstrated the importance of social trust in relation to gratification delay. The support of a new sex partner in terms of implementing all three safer-sex behaviors was mentioned positively by most respondents. The importance of support has also been highlighted by Romer (2010), who showed that young adults are especially vulnerable due to a lack of experience with novel adult behavior. Our results show that respondents low in intention to perform all three protective behaviors did not identify any facilitating factors for these behaviors as opposed to respondents high in intention to perform all three behaviors who identified support from and trust in their new sex partner as facilitating factors.

One limitation of this study is that, despite anonymity, respondents may have felt the need to answer the open questions in a manner that is viewed favorably by others, a phenomenon known as the social desirability bias (Crowne \& Marlowe, 1960). Second, the term "risky sex practices" was predefined as unprotected insertive anal sex or rough sexual practices that could evoke minor vaginal lesions, leaving little room for personal 
interpretation of the term by participants. However, we cannot completely exclude the possibility that other risky sex practices, such as rough oral sex, were not taken into consideration by participants. Lastly, answers to the open questions were translated into English by the first author. This may have led to a loss of fine linguistic nuances that exist in one language but are hard to translate into another language. Although great care was taken over the translations, we cannot exclude the possibility that some nuances may have been lost during this process.

\section{Conclusion}

Engaging in less risky sex acts, asking a new sex partner about his/her sexual health status, and using condoms (at least during first-time sex with a new sex partner) were all identified as behaviors which protect against the contraction of STIs. While condom use was often perceived as a pleasure-reducing "necessary evil," the idea of engaging in less risky sex acts was repeatedly associated with boredom and rejected on the grounds of reduced hedonic experience. Furthermore, asking a new sex partner about his/her sexual health status was often perceived as embarrassing and unpleasant, leading to widespread rejection mostly within subgroups reporting low intentions to perform all three protective behaviors. Peer influence was not reported to be a very influential factor, but parents and health care professionals were identified as normative referents regarding the implementation of the three target safer-sex behaviors.

Three major recommendations emerge from this work. First, the referent power of health professionals, particularly physicians, could be better utilized in safer-sex education programs. Raising awareness, especially among health professionals, of their normative role associated with engaging in less risky sex acts and asking a new sex partner about his/her sexual health status, could help increase the effectiveness of safer-sex programs. Second, more research is needed on how parents and family - as normative referents who approve of the implementation of all three behaviors - can be more involved in safer-sex education programs, especially those programs targeting young adults who are low in intention to implement protective behaviors. Given that new sex partners who disapprove of safer-sex behaviors were identified as strong normative referents by young adults low in intention to perform protective behaviors, parents and family could provide an important counterbalance in terms of helping young adults to implement safer-sex behaviors. Third, although respondents with high and low intention to perform all three behaviors identified these behaviors as providing protection against STIs, respondents with low intention focused mainly on factors complicating their implementation, as compared to respondents with high intention who had a more balanced assessment. We recommend that more research is conducted which addresses how to shift focus away from complicating factors and towards facilitating factors. This may encourage young adults to engage in protective behaviors, particularly those low in intention to do so. 



\section{Chapter Three}

Gaming For Safer-Sex: Young German and Turkish People Report no Specific CultureRelated Preferences Towards Educational Games Promoting Safer Sex

Based on:

Brüll, P., Ruiter, R. A. C., Wiers, R. W., \& Kok, G. (2016). Gaming for Safer Sex: Young German and Turkish People Report No Specific Culture-Related Preferences Toward Educational Games Promoting Safer Sex. Games for Health Journal, 5(6). http://doi.org/10.1089/g4h.2016.0016 


\section{Abstract}

Comprehensive sex education programs specifically designed for adolescents and young adults that take into account gender norms and cultural background have shown promise as a means of countering the high STI rate in young people. Recently, digital gaming interventions delivered on computers or mobile devices have emerged as another way to promote safer-sex behavior in a young population. Tailoring these computer-based interventions to their target population has been recognized to increase positive behavior outcomes. In this qualitative study, we investigated whether young female and male adults from two different cultural backgrounds (all living in Germany) would have different preferences and needs in relation to an educational game promoting safer-sex. We conducted four semi-structured focus group interviews comprising open-ended questions with male and female participants who had either a German or a Turkish background. In total, 20 individuals participated, aged between 18 and 22 years, from two socially diverse and ethnically mixed vocational schools in Germany. Independent of cultural background and gender, participants preferred a "real world" design with a firstperson visual perspective over a fantasy-like third-person perspective. Furthermore, they preferred highly customizable avatars. All participants mentioned the importance of including an alcohol intoxicated avatar and most participants wanted there to be additional information available about various safer-sex approaches and about the use of different barrier protection methods. Males and females reported similar preferences for the design of an educational game promoting safer sex, with the only difference being exactly how the topic of having sexual intercourse should be addressed in the game. Males preferred a direct approach, whereas females had a preference for treating this subject more sympathetically. Educational games offer anonymity and can provide young people across different cultural backgrounds with gender-tailored opportunities to experiment with specific safer-sex precautions in a nonthreatening virtual environment, free from unwanted parental control and peer monitoring. 


\section{Introduction}

Every year, a quarter of a billion young people aged 15-24 years acquire a curable sexually transmitted infection (STI) for the first time. This accounts for nearly half of all new curable STIs (World Health Organization, 2013). In 2012, with 900,000 people infected with human immunodeficiency virus (HIV) in Western and Central Europe, the prevalence of newly infected 15 -24-year olds was $0.2 \%$ for females $(1,800)$ and $0.3 \%(2,700)$ for males (Unaids, 2013). Tried and tested approaches for improving young people's sexual decision making in such a way that resulting behavior does not harm their health but keeps them sexually healthy - and thereby reducing the rate of new STIs - include curriculumbased sex and HIV education programs (Carter, 2012; Kirby, 2008; Kirby \& Laris, 2009; Kirby et al., 2007; Kohler et al., 2008; Schaalma, Abraham, Gillmore, \& Kok, 2004; StangerHall \& Hall, 2011). Developing programs for young people has proven to be a promising way of lowering the high infection rate seen in this population (Catalano et al., 2012; World Health Organization, 2010).

The recognition that gender norms (Rogow et al., 2013) and cultural factors (Schalet, 2011) influence the sexual decision-making process of young people can further improve the impact of sex education (Bell et al., 2012). In this paper, we associate ethnic identity with boundaries that determine "who is a member and who is not and designate which ethnic categories are available for individual identification at a particular time and place" (Hughey, 1998, p. 239). In this sense, boundaries emerging out of ethnic identity help to answer the question: Who are we? In contrast, culture is seen as a conceptual construction that emerges from individual action, group action, and individual and group interactions within larger society (Ashcraft, 2003), helping to answer the question: What are we? (Harper, 2007). The way females learn about sex and sexuality often differs from the way males learn about these issues, with, for example, females preferring intimate talks with parents, especially mothers, and males preferring peers and mostly exclude adults for intimate talks about sexuality (World Health Organization, 2010). This leads to genderspecific expectations related to sexual intercourse - and therefore a need for gendertailored sex education programs (Nagel, 1994). Furthermore, integrating popular culture, such as online games, into sex education efforts - as well as responding to a need for information that integrates culture-specific expectancies - helps to ensure that programs are grounded in the lives and realities of young people (Measor, 2004; Murdock \& Kluckhohn, 1962; Rawson \& Liamputtong, 2010; Schmitt, 2005; Singer, 2010).

In recent years, digital gaming interventions have emerged as a novel way of making developmentally and culturally appropriate interventions available to a population of young people (Enah et al., 2013). These so-called educational games are interventions delivered by computers or mobile devices that aim to educate or promote behavior change while at the same time being enjoyable and intrinsically motivating (Graesser et al., 2009; Pamela M. Kato, 2010; Prensky, 2007). Learning effects are fostered by utilizing immersion techniques (whereby a player becomes fully absorbed in the play), by 
establishing flow (a state of highly focused concentration), and by meeting the specific individual's desire to become proficient in the game (Annetta, 2010; Boyle et al., 2012; Connolly et al., 2012; Lu et al., 2012). Delivering interventions promoting behaviorchange in an educational game environment has been shown to facilitate positive outcomes in participants, such as increased motivation and enjoyment to take part in an intervention (Hamari et al., 2014). However, in a recent meta-analysis (DeSmet et al., 2014b), DeSmet et al. reported that educational games failed to induce a long-term effect on behavior, and stressed the importance of tailoring a game not only towards behavioral outcomes but also taking into account "both socio-demographic information (e.g. age, gender, body frame) and behavioral change needs (e.g. current level of lifestyle adoption, already acquired knowledge, stages of change, or motivation)". It has been recognized that this kind of tailoring of computer-based interventions can lead to engaging and immersive learning experiences that are effective in creating positive behavior outcomes, such as increased fruit and vegetable intake, and decreased calorie consumption (Starks, 2014). Tailoring game content in this way has been shown to be particularly effective in educational games for sexual health promotion (Noar et al., 2009; Portnoy et al., 2008).

In the present study, our goal was to identify content- and design-related preferences among future players of an educational game promoting safer sex that we are currently developing for young people. Furthermore, we wanted to investigate whether these game-related preferences would differ depending on gender and cultural background. It has been reported that preferences for safer-sex programs depend on both gender and culture (Fentahun, Assefa, Alemseged, \& Ambaw, 2012; Lakon \& Hipp, 2014; Newby, Wallace, Dunn, \& Brown, 2012), even if these differences might be much smaller than common understanding would suggest. For example, a written survey study conducted in China (among 440 college students aged between 19 and 24 years) which asked about preferred sources for sex education showed that females favored reading material, classroom lectures and their parents as sources of information, whereas males preferred friends, the internet and personal sexual experience as primary sources (Li, Cottrell, Wagner, \& Ban, 2004). Coleman and Testa (2007) conducted a cross-sectional survey among an ethnically diverse sample of 3,007 adolescents aged between 15 and 18 years attending schools in London, UK, about their preferred topics in relation to sex education programs. Females, in general, favored learning about emotions, relationships, and contraception, preferably delivered by someone of the same sex. Black students favored learning more about biological and cultural influences, such as the reproductive cycle or culture-specific role distributions, as well as sexual behavior and STIs, with black male students showing a preference for family-based information, preferably delivered by someone of the same ethnic background. In contrast, Asian students showed a stronger interest in information about STIs and contraception, while preferring to keep sex education out of the family household. With regard to such different attitudes and expectations being influenced by gender and culture, the present study firstly focused on identifying potential game-related content and design preferences of future players. Secondly, we 
explored whether these game-related preferences would differ depending on the gender and cultural background (German or Turkish) of the players.

\section{Materials and Methods}

\section{Participants and procedure}

Four focus groups were conducted with a total number of 20 participants, all aged between 18 and 22 years (Table 1). All participants were recruited from two vocational schools in Germany that are attended by students with diverse social backgrounds (e.g., socio-economic status) and different ethnic identities (e.g., African, Turkish, German). All procedures followed were in accordance with national and international ethical standards. Following approval from the institutional ethics committee, researchers informed participants about the study in general terms during a short visit to their classroom. Furthermore, posters and flyers in common areas such as the refectory and entry hall also displayed a quick response code (a so-called QR-code consisting of a matrix barcode that can be scanned by smartphones and links to a website) that allowed participants to get more information about the study. Only potential participants who felt closely related to a German or a Turkish cultural background and were born to parents who have either a German or a Turkish cultural background were invited to sign-up. After giving their written informed consent online, participants could sign-up for the study. They then received an email containing more details about the date and location of their specific focus group interview. A short demographic survey with open-ended questions asked participants to identify their age, gender identity, primary cultural background (German or Turkish) and the name of the vocational school that each participant currently attended. Participants were allowed to quit the study at any time (without providing a reason and without any consequence). 
Table 1. Characteristics of the Study Sample $(n=20)$.

\begin{tabular}{|c|c|c|c|}
\hline Participants & Age (years) & Gender & Cultural background \\
\hline \multicolumn{4}{|c|}{ Recruitment at vocational school 1} \\
\hline Participant 1 & 19 & male & German \\
\hline Participant 2 & 21 & male & German \\
\hline Participant 3 & 18 & male & German \\
\hline Participant 4 & 18 & male & Turkish \\
\hline Participant 5 & 22 & male & Turkish \\
\hline Participant 6 & 19 & male & Turkish \\
\hline Participant 7 & 18 & female & German \\
\hline Participant 8 & 20 & female & German \\
\hline Participant 9 & 19 & female & Turkish \\
\hline Participant 10 & 21 & female & Turkish \\
\hline \multicolumn{4}{|c|}{ Recruitment at vocational school 2} \\
\hline Participant 11 & 18 & male & German \\
\hline Participant 12 & 18 & male & German \\
\hline Participant 13 & 21 & male & German \\
\hline Participant 14 & 18 & male & Turkish \\
\hline Participant 15 & 19 & male & Turkish \\
\hline Participant 16 & 22 & female & German \\
\hline Participant 17 & 18 & female & German \\
\hline Participant 18 & 18 & female & German \\
\hline Participant 19 & 19 & female & Turkish \\
\hline Participant 20 & 21 & female & Turkish \\
\hline
\end{tabular}

Although a great variety of groups with different cultural backgrounds can be found in Germany, people with a Turkish cultural background represent by far the largest group, comprising 3.36\% of the German population in 2012. Turkish culture differs from German culture in aspects such as the form of relationship between the individual and the collectivity (with Turkish culture tending towards collectivism versus German culture tending to individualism), the importance of family ties (with Turkish culture emphasizing the importance of family ties stronger than German culture), the number of children (with Turkish families having more children than German families), the definition of gender roles (with Turkish culture valuing masculinity over femininity compared to a more balanced value in German culture), and tolerance of ambiguity and uncertainty (where uncertainty avoidance is higher in Turkish culture compared to German culture) (Statistisches Bundesamt, 2015). Young people with either a German or a Turkish cultural background, who were fluent in German, were included in the present study. There were 11 male participants aged between 18 and 22 years $(M=1 \quad 9.80,=1.4$ ) from which five had a Turkish cultural background and six had a German cultural background. Out of nine female participants aged between 18 and 22 years ( $M=19.50,=1.5$ ), four had a Tuski cultural background, and five had a German cultural background. 


\section{Study setting}

Every focus group had the same moderator and was held in the evening at one of the schools that participants attended. Participants gave their consent and received a gift voucher of $€ 25$, for their participation. Focus groups were divided by gender and cultural background, resulting in four different groups: males with a Turkish cultural background, males with a German cultural background, females with a Turkish cultural background, and females with a German cultural background (see Table 2). Interviews were based on a semi-structured protocol containing open-ended questions which assessed three factors related to the design, and three factors concerning the content of the game. Questions about game design were: "What do you want a game about safer-sex education to look like?", "What kind of feel should the game have?", and "How would you want to be represented as a player in that game?" Content-related questions were: "What kind of content should be covered by the game?", "Are there any topics that you feel strongly should be included?", and "What specific aspects of safer-sex education do you think should be covered by the game, or should not be covered at all?"

Table 2. Composition of the Different Focus Groups.

\begin{tabular}{lll}
\hline Focus groups & Mean age in years $(S D)$ & Participants \\
\hline Group 1 & $19.2(1.6)$ & Participant 4 \\
Males with Turkish cultural background $(n=5)$ & Participant 5 \\
& Participant 6 \\
Group 2 & Participant 14 \\
Males with German cultural background $(n=6)$ & Participant 15 \\
& Participant 1 \\
& $19.7(1.4)$ & Participant 2 \\
Group 3 & & Participant 3 \\
Females with German cultural background $(n=5)$ & Participant 11 \\
Females with Turkish cultural background $(n=4)$ & Participant 12 \\
& & Participant 13 \\
& & Participant 7 \\
& & Participant 8 \\
& & Participant 16
\end{tabular}

All interviews were conducted in German and lasted between 45 minutes and one hour. All focus group sessions were audio recorded, and during the sessions observational 
notes were taken, focusing on any strongly stated comments made by participants, their body language, and any non-verbal activity such as eye contact between certain participants. All sessions started with an introduction to the objectives of the study (including a short introduction to serious games), and information about the role of the participants during the focus group. Participants introduced themselves using only their real or fictitious first names to increase confidentiality. At the end of the session, the answers to the questions were summarized, and participants were asked to provide feedback in order to confirm the accuracy of the findings.

\section{Data processing and analysis}

Interviews were conducted until data saturation (the point at which no new information emerges) was reached. The interviews were recorded with a digital voice recorder and transcribed verbatim by the first author. During transcription, all identifying and personal information was deleted. The final transcripts - as well as the audio recordings - were then reviewed by the first author and by an independent researcher, in order to ensure that the focus group interviews were transcribed completely and accurately. Transcripts were imported into the qualitative data management software NVivo version 10 (QSR, Doncaster, Australia) and analyzed by the first author, who conducted a thematic analysis (Braun \& Clarke, 2006) in which themes were established and explored. An inductive process was used to derive any sub-themes from the main themes. A sample of $30 \%$ of the data was coded by a second researcher who was independent from the first author. The initial coding was compared, reviewed and in the few instances where differences were found, refined in order to result in a more representative coding. Having established consensus on how the data were coded, the codebook was updated, and the transcripts were coded by the first author. Results are structured according to the themes that emerged from this analysis. Quotations representing themes are included to demonstrate study findings. These quotations were translated from German to English by the first author who is native German-speaking and experienced with translating German into English and then back-translated to German by an independent researcher (also a native German speaker) to ensure the accuracy of the English translation. Table 3 provides an overview of themes and sub-themes that emerged from the focus groups, based on how frequently they were mentioned. 
Table 3. Focus Group Themes and Sub-themes Based on Frequency of Mention.

\begin{tabular}{lll}
\hline Focus group question & Theme & Subthemes* \\
\hline General look of the game & Realistic real-world design & None identified \\
General feel of the game & & None identified \\
Representation of the player & Single-player & Security \\
& Multi-player & Realistic \\
& Avatar & Uncertainty \\
& & Identity \\
Content covered & Approach to sex & Realistic \\
& & Directness \\
& & Realistic \\
Important topics & Health-related contexts & Anonymity \\
Specific aspects & & Realistic \\
& Intoxication & Recreational \\
\hline
\end{tabular}

* Sub-themes are presented in descending order according to how frequently they were mentioned.

\section{Results}

\section{Realistic real-world design}

There was consensus across all four focus groups that the overall design and layout of the educational game should be similar to a real-world setting. In particular, participants were unequivocal in their rejection of fantasy or fairytale-like designs. One 22-year-old German male stated that "the design should reflect the real world. No fairytale-like sceneries. Just like a real city or like a real room, something that I can recognize as being realistic". Moreover, participants did not approve of designs linked to pre-adolescent age ranges, such as avatars with unnatural skin colors (e.g., light blue) or unnatural environments (e.g., fantasy landscapes). In relation to one such design, an 18-year-old Turkish male responded: "Oh no, not such a child-like setting. It needs to be real, well, realistic, like avatars looking like real people in a real environment".

\section{Single-player or multi-player game}

There were no specific preferences among male participants toward having only nonplayer characters controlled by computer artificial intelligence (single-player) or avatars controlled by real online players (multi-player) to interact with. In general, female participants also embraced the possibility of a multi-player design. One 19-year-old Turkish female stated: "There should be real players, well, an online game where other avatars are 
controlled by real people. That would be exciting. And you could meet online like knowing that there is really someone controlling the avatar that you don't know". However, there was no overall consensus, as some female participants were more in favor of a singleplayer game. A 22-year-old Turkish girl said that "there should also be a possibility to meet computer avatars that are not linked to a real person. Like having places where only computer avatars are present and other places such as specific bars or clubs where avatars are controlled by real people". The main objection against a multi-player game was the perceived uncertainty regarding who would be the real person behind an avatar in a multi-player environment. One 21-year-old German female mentioned: "You never know who is behind a certain avatar, like a guy over 60 or so. I would feel like prey. There should be no chat option, only predefined dialogues".

\section{Avatars}

The look and feel of avatars was important for participants across all focus groups. They preferred to control their avatar from a first-person visual perspective (i.e., seeing through the eyes of the avatar) as compared to an elevated third-person visual perspective. A 17year-old German male stated: "To see my avatar from above is not appealing. He needs to have a character, well, the one I gave to him". A 19-year-old German female reported: "The perspective is also important to me. Looking from above down on my avatar just feels strange. I need to see her at the same level, like in ego-shooter games". Furthermore, all participants wanted to be able to adapt the avatar to incorporate their own identity. One 22-year-old Turkish female said: "I should be able to adapt the avatar to my needs, like hair color, outfit, age, and so on." A 22-year-old Turkish male stated: "To identify with my avatar, I need to be able to give him some character traits that I find important. Viewing him from above is a no-go! I need to see him face-to-face or in ego-shooter style". Moreover, across all groups, participants agreed that the avatars would need to look human-like. An 18-year-old Turkish female said: "I can't imagine like fantasy characters having sex! No, that would be ridiculous. They need to look like humans". A 22-year-old German girl mentioned: "I need to identify with my avatar. It is like if she does something, it needs to be as if I did it. They need to look somewhat realistic". A 17-year-old Turkish male said: "Avatars should be able to form groups, like cliques, and go out together and have fun. Like in real life." One 21-year-old German girl mentioned: "Avatars should also represent people from other ethnicities. What if I have a preference for Black men? I should be able to find a corresponding avatar, too. Avatars should be multicultural and multiethnic".

\section{Approach to sex}

There were differing views across gender about how sexual intercourse should be integrated into the game. Asked about it, male participants opted for a straightforward implementation. An 18-year-old Turkish male mentioned that "avatars should have sexual diseases. Then you would need to decide if you want to have sex or not". Some female 
participants preferred a softer and more indirect approach, emphasizing the sensual experience instead of focusing on the physical intercourse while still integrating the consequences of sexual behavior. A 17-year-old Turkish girl said: "It would be nice to approach sex in a more subtle way, like not making too much fuss about it." Other female participants were in favor of having a realistic approach. One 19-year-old German girl said: "Well, I would make it more realistic, I mean if it is from 16 onwards, I mean everyone knows what a naked man or a naked woman looks like. I don't think that would be a problem". Another 19-year-old Turkish girl mentioned that "choosing between different sexual positions would be important. You could test out things you don't know yet using your avatar". Some female participants mentioned that avatars should also probe players 'commitment to safer-sex. A 21-year-old German girl said: "But there should be questions during the game, like, 'do you want to have sex with him?', 'cause you don't have any condoms with you, you know. You would ask that question to yourself if you were in that kind of situation". Across all focus groups, participants wanted to have avatars that could get STIs if they had unsafe sex. A 22-year-old German girl stated: "Yes, and they should also be able to get diseases. If they don't take care, avatars should run the risk of infecting themselves and even of dying". Moreover, participants valued the anonymity provided by games. Especially when touching upon a sensitive and private topic such as sexual activities, having the freedom to experiment with the different consequences associated with their sexual decision making in a setting free from peer monitoring and - most importantly - without facing negative consequences in real life, was important to the participants. As one 19-year-old Turkish girl said: "It is OK to have a straightforward approach to sex in the game. This allows me to test things without facing negative consequences and without my friends knowing what kind of fantasies I might have".

\section{Health-related contexts}

When asked about specific points the game should focus on, males stated that they would like to have some kind of contest in the game. One 22-year-old German male said: "Some action would be nice, like fighting rivals." Female participants tended to opt for more everyday scenarios. A 22-year-old Turkish female stated: "Including daily activities seems important to me, like it would be a normal day and then you go out at night, which means that the male avatar needs to wait until I am dressed up or until I have finished dinner. Or he needs to be patient until I decide that I am ready". An 18-year-old Turkish female said that "consulting a physician would be cool. Like, he then tells you that you are ill or he just gives you some pills" and an 18-year-old Turkish male said: "It should be fun, too! Maybe a physician challenges us with sex-related questions, and we need to solve them, like finding out the correct answers by asking others or by just trying things out". A 19-year-old German girl mentioned: "And it should be possible to get into precarious situations during the game - and then play through them." One 21-year-old German girl summarized this nicely: "It should be like in real life; like situations, you could really experience." 


\section{Intoxication}

Alcohol consumption was identified as an important variable. One 22-year-old Turkish girl said: "Alcohol or drugs should be in there. I mean, that is a reason why many people end up having sex. If the avatar takes drugs or drinks too much, the screen could be blurry or shaky, or the avatar could not correctly execute our commands any longer". A 17-yearold Turkish male reiterated this idea by stating: "Alcohol is important! If my avatar is drunk, he should be somehow out of my control".

\section{Imparting knowledge}

Some participants wanted to get more information about safer-sex precautions. A 17year-old German male said: "Combining avatar decisions such as having unsafe sex with explanations about the risks would be helpful." A 19-year-old Turkish male mentioned that "there could be quizzes about safer-sex, like one playing against another and whoever knows the right answer wins." One 22-year-old German male said: "I would like to have an info board, like a virtual info center that provides me with all the information I need to know. Like a 'sex master' who tells me about fun and risks related to certain practices". Some participants asked for educational scenarios explaining the use of specific barrier protection methods. An 18-year-old Turkish girl said: "Like being told by a virtual physician like how to use a contraceptive coil, how to insert it, well, how all this stuff works. But only briefly, like a video clip". A 19-year-old German girl mentioned: "There should be short video clips explaining like how to use a condom. The avatars could show that". A 22year-old German girl confirmed the need to impart knowledge related to the handling of contraceptives: "Showing how to use contraceptives is important, like including pictures or giving tips."

Besides gender-related differences regarding the approach to sex, no other genderor culture-related differences could be identified. Participants showed homogeneous expectations toward game design and overall content.

\section{Discussion}

The findings from the present study demonstrate that the design preferences of young people toward an educational game promoting safer sex did not differ depending on their cultural background. However, some gender variation was found related to specific components of the game.

All participants opted for a design that reflects a real-world scenario and rejected all designs reminiscent of a fantasy world. Young female adults, independent of their cultural background, expressed their desire to include daily activities, such as putting makeup on or dressing their avatars. This preference for using a realistic setting in sex education programs aimed towards young people has been reported previously (Coleman \& Testa, 2007; 
Newby et al., 2012) and is also in line with recent theories of gamification (Ferrara, 2013). Young male adults across all groups also opted for a realistic setting but wanted to include some action modules (e.g., some sort of contest) to increase gaming enjoyment.

While male participants did not express a preference for either a multi-player or a single-player game, female participants preferred the multi-player option because this allows for a real avatar-induced virtual social contact. Their main concern about this option - namely uncertainty about the real gender and motivation of the actual person behind the avatar - has been addressed in previous research. Crowe and Watts (Crowe \& Watts, 2012) showed that proclaimed identities within virtual social systems are not reliable. A more recent study emphasized that men who use a female avatar tend to align with the social norms and rules related to the gender of their avatar (Martey, StromerGalley, Banks, Wu, \& Consalvo, 2014). However, in a multi-player game, avatars remain virtual agents that do not necessarily reflect the actual gender and motivation of the real individuals who operate them.

Notwithstanding these findings, one important way of creating real-world scenarios is to increase self-identification with the virtual avatar that is supposed to depict the player. All participants in our study preferred a first-person visual perspective. This is not surprising, considering that the detection and recognition of social interactions are facilitated in this perspective (Fathi, Hodgins, \& Rehg, 2012). Moreover, as opposed to a thirdperson perspective, the first-person perspective is centered upon the player's own body and is perceived in an egocentric reference frame (Vogeley et al., 2004). Such a metarepresentation of mental and bodily states is a prerequisite to the induction of human selfconsciousness as related to a virtual avatar (Vogeley \& Fink, 2003) and when attempting to increase the player-avatar identification (Spanlang, Normand, Giannopoulos, \& Slater, 2010). This is also reflected in the importance participants placed on being able to customize their avatar. An avatar that allows for a high degree of customization was preferred over an avatar with a low degree of customization, or a pre-defined avatar. High avatar-player similarity determines the player's identification with the avatar, which in turn leads to more enjoyment of the game (Trepte \& Reinecke, 2010). Personalization of an avatar has also been shown to increase embodied perception (Ducheneaut, Wen, Yee, \& Wadley, 2009; You \& Sundar, 2013) so that the more closely an avatar resembles its user, or has been created according to the user's specifications, the more likely it is that the user will hold positive attitudes toward this avatar (Suh, Kim, \& Suh, 2011). Within the framework of safer-sex promotion, all participants across gender and cultural background showed a clear preference for highly customizable avatars.

Content-wise, young people's preferences did not differ depending on their cultural background. The only gender variation observed was related to how the topic of having sexual intercourse should be approached by the game.

Across all focus groups, participants advocated the inclusion of an alcohol intoxicated avatar, because "that's when you end up having sex with unknown people," as one 22year-old German female mentioned during the discussion. Alcohol consumption remains 
related to higher rates of sexual risk-taking (B. T. Jones, Jones, Thomas, \& Piper, 2003; MacDonald, Zanna, \& Fong, 1996; Patrick \& Maggs, 2009; White, Fleming, Catalano, \& Bailey, 2009) and implementing the option to select a drunk-avatar-condition in an educational game that aims to promote safer-sex practices could allow young people to experience reduced volitional control in a safe environment. Integrating these preferences in future games appears to be justified, especially as it has been recommended that the design of these kinds of educational games need to take into account the players' expectations with regard to the characteristics, goals, rules, and challenges of the game (Charsky, 2010; Kankaanranta \& Neittaanmki, 2009; Michael \& Chen, 2005). This is particularly relevant when trying to engage hard-to-reach populations who may be attracted to educational games because of a pre-existing enjoyment of gaming (Rideout, Roberts, \& Foehr, 2005).

Previous studies have observed that, as compared to males, female adolescents express a greater desire for safer-sex information covering multiple topics (Forrest, Strange, Oakley, \& The RIPPLE Study Team, 2004; Newby et al., 2012), and that preferences across topics differ according to ethnic or cultural group affiliation (Coleman \& Testa, 2007; Smerecnik, Schaalma, Kok, Meijer, \& Poelman, 2010). We cannot support these findings as no differences related to gender or cultural background have been observed with regard to safer-sex information. Across all focus groups, participants expressed their desire to be informed about various safer-sex approaches and about the specific use of different barrier protection methods or contraceptives. The only gender-related difference we found was that males preferred a more direct approach when it comes to introducing sexual intercourse in the game, whereas female participants favored a slightly softer approach that would be embedded in a narrative but nevertheless needed to remain realistic. This need for a narrative is beneficial and has been identified by Kapp (2012) as providing the player with a vicarious learning experience.

It is possible that this openness across gender and culture toward different safer-sex methods and precautions could be explained by the unique characteristics of educational games. Adolescence and young adulthood are times for developing a new sense of self and offer opportunities to influence behavior that supports positive long-term consequences (Dahl, 2004). Young people need opportunities to observe and reflect on the possible consequences of sexual risk behaviors in a safe and nonthreatening environment. Educational games meet this need and allow for an exploration of new activities. The resulting consequences of this exploration take the form of reward (e.g., gaming points) or loss (e.g., a diseased avatar). What's more, this exploration takes place in a private setting, free from parental or peer influence (Enah et al., 2013). Educational games tailored towards the needs and expectations of young people could offer them the necessary privacy to explore safer-sex practices not commonly used within their social environment.

This study has some limitations. Ethnic and cultural group categorization is complex. Cultural groups need to be homogeneous across different religious and social subgroups, 
whereas ethnic groups need to reflect not only a common descent but also a shared culture and history that is more often dynamic than fixed. It is acknowledged that cultural and ethnic categorization might obscure diversities within groups and therefore reinforce unhelpful stereotypes. Therefore, care should be taken in applying these results to various cultural or ethnic subgroups.

Furthermore, this study presents only a limited sample of young people from one of just two different cultural backgrounds. The cultural capital of young people raised in Germany by their Turkish families might be influenced by their mixed cultural environment (Marjoribanks, 1997; Xiao, 2008; Xu, Bekteshi, \& Tran, 2010). It is possible, therefore, that different results might be found in relation to young Turkish adults born and raised in Turkey. Although we included the interviews of young adults whose views are not normally considered (i.e., young adults from a Turkish cultural background), the rather small sample size makes it difficult to determine whether data saturation was finally reached. This also holds true for males versus females as our limited sample of participants could lack data present in a larger sample. However, we included information about dissenters to determine within-group saturation, as this would increase the descriptive validity, interpretive validity and theoretical validity associated with the emergent themes. We further monitored the quality of words to assess across-group saturation. However, due to our small sample size, we may have missed information that would have emerged had we had more focus groups.

Participants were told that the study objective was to identify how a serious game that promotes safer-sex behavior should look and feel like. Therefore, we cannot exclude the possibility that an expectancy bias (i.e., a response variance due to varying expectations of the purpose of a task by participants so that their own view or opinion is not truly reflected) may have occurred (Gomm, 2009). However, we were careful to design our questions in a way that avoided creating expectations that would inadvertently shape responses. We further standardized the recruitment material and used a standard opening statement (read out by the moderator at the beginning of the sessions) which clarified both process and roles. Moreover, participants introduced themselves using only their first names to increase confidentiality. However, because some participants attended the same school, we cannot exclude the possibility that some might know each other, and this could have led to an acquaintance bias (i.e., individuals who know each other may influence each other's responses) (March, 2013). Even if we did not identify any biased responses, we cannot exclude the possibility that such a bias may have occurred.

Lastly, transcripts of recordings were reviewed by only one additional researcher for thematic analysis and translated into English by the first author. This might have led to missed codes and to a loss of fine linguistic nuances that exist in one language but are not exactly transferable into another language. Although great care was taken over the coding and translations, we cannot exclude the possibility that some codes and linguistic nuances may have been lost during this process. 


\section{Conclusion}

All participants preferred a real-world design with a first-person visual perspective, and female participants, in addition, preferred a multi-player option. Great emphasis was placed on the importance of highly customizable avatars. Participants said that they would like to have information modules available throughout the game to explain various safer-sex approaches and the use of different barrier protection methods. Across all focus groups, participants stated the importance of including an alcohol intoxicated avatar to increase awareness about the loss of control that can occur while in an intoxicated state. Young people's preferences toward an educational game promoting safer sex were identified as being mostly independent of their cultural background. Only female participants preferred a more indirect approach towards topics related to sexual intercourse, whereas male participants preferred a more direct approach. In summary, educational games profit from the anonymity they provide. In the present study, we established that such a game could offer young people with different cultural backgrounds (in this case German and Turkish) gender-tailored opportunities to experiment with specific safer-sex precautions in a non-threatening virtual world that is free from parental or peer monitoring. 


\section{Chapter Four}

\section{Different Levels of Sexual Arousal - Friends or Foes in Sexual Decision Making?}

arousal - friends or foes in sexual decision making? 


\section{Abstract}

Young people are especially prone to acquiring sexually transmitted infections (STIs). In addition to condom use with a new sex partner, asking about his/her sexual health status and refraining from risky sex practices both significantly reduce the risk of acquiring STIs. However, as a prominent motivational force, sexual arousal can affect individual preferences and behaviors in such a way that judgment and risk assessment are compromised. In this study, we examined the effect of sexual arousal on implicit and explicit attitudes, perceived norms, perceived behavioral control and intentions towards safer-sex behaviors. Furthermore, we explored the role of sexual arousal on self-reported positive and negative life expectations, decisional preferences in different life areas (such as health care prevention), and domain-specific risk perceptions. Finally, we explored the influence of sexual arousal on executive functions as measured by working memory capacity. One hundred and sixty-nine sexually active participants who were not in a relationship, aged between 16 and 22 years, participated in this online study that consisted of a withinsubject manipulation of sexual arousal. We did not find an effect of sexual arousal on lifestyle inventory items, risk perception, perceived norms, perceived behavioral control and intentions towards the three safer-sex behaviors of interest, or on the measure of working memory capacity. Instead, we found an effect on attitudes and life expectations. Our results suggest that sexual arousal can both reduce but also support different kinds of health-sustaining sexual behavior, as sexual arousal resulted in less positive attitudes towards condom use whereas it promoted attitudes towards asking about the sexual health of a new sex partner. Furthermore, our data indicate that self-reported positive and negative life expectations were amplified during sexual arousal. A better understanding of the influence of sexual arousal could be used to improve the effectiveness of safersex education programs. 


\section{Introduction}

Each year, half a billion new curable sexually transmitted infections (STIs) are estimated to occur worldwide (World Health Organization, 2013). Young people aged 15-24 years (Sawyer et al., 2012) acquire nearly half of these infections (Centers for Disease Control, 2015) and the relative prevalence of human immunodeficiency virus (HIV) in this age group remains high (Centers for Disease Control, 2015; Piot et al., 2015; World Health Organization, 2014). Young people are especially prone to both acquiring STIs and to infect others. This is mostly due to reduced access to quality STI prevention and health services, alongside a discomfort with using the available facilities (Centers for Disease Control, 2015; Hollingsworth et al., 2008). Sexual risk behavior, such as unprotected intercourse with an unknown sex partner, remains a problem in late adolescence and early adulthood, despite the implementation of various school-based and community-based sex education programs (Patton et al., 2016).

Most sex education programs - as well as research on sexual risk behavior - is focused on the use of barrier protection, such as condoms. In the present study, we broaden this focus by also addressing whether individuals ask about a new sex partner's sexual health status, and refrain from risky sexual practices, such as penetrative anal sex. These three sexual health-related protective behaviors have been shown to reduce the risk of acquiring STIs (Varghese et al., 2002). Understanding the determinants of these behaviors - and encouraging these behaviors - could be a promising approach when it comes to the reduction of HIV infection rates among young adults. To illustrate the importance of this the relative risk of HIV infection is reduced 47-fold by choosing a sex partner who has tested negative (as compared to an untested sex partner). Using condoms further reduces the risk 20-fold; and abstinence from penetrative anal sex in favor of penetrative fellatio reduces the risk 13-fold (Kim \& Koopman, 2012; Varghese et al., 2002).

Studying the determinants of safer-sex behavior is mostly carried out while participants are in a sexually non-aroused state. In the present study, we focused on how sexual arousal might influence explicit and implicit attitudes towards the three protective behaviors of interest. From an evolutionary perspective, sex drive may increase the chances of having sex, but also contribute to the problem of unprotected sexual intercourse. In fact, sexual arousal can influence individuals' preferences and behaviors in such a way that judgment and decision making become riskier. An example of this can be seen in research carried out by Ariely and Loewenstein (2006), who showed that sexual arousal had a strong impact on how appealing male undergraduate students found a wide range of sexual stimuli and activities (such as body weight or anal sex), and the willingness to engage in unsafe sex. Their study showed that judgment and decision making were strongly influenced by sexual arousal, making gratifying but risky choices more attractive and appealing in an aroused as compared to a non-aroused state. Yang et al. ( 2012) suggested that people tend to be more likely to behave healthily if they are not in a state of arousal. They introduced a so-called hot-cold decision triangle as a framework for 
healthier choices which can support people to embrace colder (non-aroused) states during reasoning. They suggest that external motivations - such as policy-level interventions - support individuals in making decisions predominantly in a cold rather than a hot state. However, sexual decision making happens mostly in an aroused state which has an impact on risk-taking and intentions to engage in risky sexual behavior (Skakoon-Sparling \& Cramer, 2016; Skakoon-Sparling, Cramer, \& Shuper, 2016). This means that protective measures are only likely to be implemented if they are already part of a behavioral script in which intentions to implement these behaviors have been linked with specific cues in the decision-making context (e.g., Sheeran, Webb, \& Gollwitzer, 2005).

\section{Explicit and implicit determinants of behavior}

One explanatory model that can be used to identify determinants of intention to perform the three safer-sex behaviors of interest is the Reasoned Action Approach (RAA) by Fishbein and Ajzen (2010). This approach emerged out of the Theory of Reasoned Action (TRA) (Fishbein \& Ajzen, 1975) and the Theory of Planned Behavior (TPB) (Ajzen, 1985, 1991), and suggests that behavioral decisions are made based on reasoned considerations derived from available information. RAA states that behavioral intention (the readiness to engage in a behavior - hence determining the actual behavior) depends on attitude (positive or negative evaluations of behavior), perceived norms (perceived social pressure regarding the performance or non-performance of a certain behavior), and perceived behavioral control (PBC; perception of the ease or difficulty related to the performance of a certain behavior) (Fishbein \& Ajzen, 2010). However, in the heat of the moment, young people in particular seem to reason more poorly than adults (Albert \& Steinberg, 2011; Hofmann, Friese, \& Wiers, 2008; Reyna \& Farley, 2006; Steinberg, 2008, 2010; Strang, Chein, \& Steinberg, 2013; Wiers, Jong, \& Alan, 2010). To identify whether sexual arousal influences the explicit determinants attitude, perceived norms, PBC, and intention towards the three behaviors of interest, these RAA variables were assessed in both a sexually non-aroused and a sexually aroused state.

However, behavior is not exclusively predicted by explicit associations. Implicit associations are fast, activated automatically, and can influence behavior outside conscious awareness (Gawronski, Hofmann, \& Wilbur, 2006; Greenwald \& Banaji, 1995; Hahn \& Gawronski, 2014), in contrast to explicit cognitions that are slow and deliberate (e.g., Strack \& Deutsch, 2004). Research has shown that implicit cognitive factors account for unique variance (over and above explicit measures) for a variety of behaviors, including eating behavior (Friese \& Hofmann, 2009), aggression (Wiers et al., 2009), and substance use (Wiers \& Stacy, 2006). Negative implicit cognitions have also been shown to predict less consistent condom use (e.g., Grenard, Ames, \& Stacy, 2013; Stacy, Ames, Ullman, Zogg, \& Leigh, 2006) and condom use intentions in low-risk scenarios (as compared to high-risk scenarios which were better predicted by explicit attitudes) (Czopp et al., 2004). 
Working memory (WM) can be defined as the ability to maintain and manipulate goalrelevant information (Baddeley, 1992, 2012; Ma, Husain, \& Bays, 2014). It is part of a collection of cognitive functions - including planning, attention, and the inhibition of inappropriate actions - that is known as executive control, and which allows people to take goal-directed actions (Baddeley \& Della Sala, 1996; Baddeley, 1998; Norman \& Shallice, 1986). Low levels of executive functions, as expressed, inter alia, in reduced WM capacity, have been shown to hamper the proper regulation of automatic impulses, for example, by shifting the regulation of drinking behavior from controlled processes towards more reward driven impulsive behavior (Friese \& Hofmann, 2009; Friese, Hofmann, \& Wänke, 2008; Grenard et al., 2008; Hofmann, Gschwendner, et al., 2008). Moreover, the association between implicit cognition and sexual behavior has been shown to be attenuated among individuals with better executive control functioning (Ames, Grenard, \& Stacy, 2013; Grenard et al., 2013). As sexual arousal is a motivational state to move toward sexual activity, and mating success is an elementary determinant of fitness that can result from uncontrolled automatic responses, as well as controlled deliberate actions, we were interested in whether WM capacity could be influenced by sexual arousal. We, therefore, assessed WM capacity in both sexually aroused and non-aroused states (MacDonald, Fong, Zanna, \& Martineau, 2000; MacDonald et al., 1996).

Demographic variables, personality, and individual differences can further influence behavior, but in a more indirect way (Montaño \& Kasprzyk, 2008). Personality traits were initially defined as stable and were assumed to be the result of biological differences or situational influences - mostly early childhood experiences - (Eysenck \& Eysenck, 1985). However, low correlations in trait-related behavior observed across different situations have led to more complex definitions that also acknowledge the important role of situational determinants (Mischel \& Shoda, 1995). The notion of risk attitude as a personality trait has undergone a similar development; it has been shown that individuals are not consistently risk-seeking (or risk-averse) across different situations (Schoemaker, 1990). Risk taking by the same person can differ across situations because the risks and benefits are perceived differently in two different domains (e.g., in relation to a recreational vs. a personal safety decision), while the individual's attitude towards perceived risks remains the same for both domains (Weber \& Hsee, 1998; Weber \& Milliman, 1997).

In the current study, our aim was to understand better how young adults' explicit and implicit cognitions towards safer-sex behaviors and more general health protecting behaviors might differ depending on their level of sexual arousal. More specifically, we were interested in whether predictors of the three mentioned safer-sex behaviors differed as a result of the participants' state level of sexual arousal (sexually aroused versus not sexually aroused). One of our questions was whether sexual arousal would influence measures of explicit and implicit determinants of sexual decision-making. Second, we were interested in whether WM capacity, as a proxy for executive functions, would be affected by sexual arousal. Third, we were interested in whether the assessment of other factors with possible long-term consequences - such as risk perception, health behavior, 
or life expectancies - would be affected by sexual arousal. Fourth, because of the widespread use of the RAA in predicting and changing behavior, we were interested in whether the predictive power of the main determinants of behavioral intention advocated by this model - namely attitude, social norms, and perceived behavioral control would be altered depending on the level of sexual arousal. The present study was approved by the institutional ethics review board.

\section{Methods}

\section{Participants}

Participants were recruited from two socially diverse and ethnically mixed vocational schools in Germany. Following approval from each institutional ethics committee and permission to use passive parental consent, researchers informed participants about the study in general terms during a short visit to their classroom. In addition to this, posters and flyers in common areas such as the refectory and entry hall displayed a quick response code (a so-called QR-code consisting of a matrix barcode that can be scanned by smartphones and links to a website) that allowed participants to find out more about the study, particularly the inclusion criteria of being heterosexual, aged between 16 and 22 years, sexually active, and not in a relationship. After giving their written informed consent, they were linked to a secured website that contained the study materials. Participants were allowed to quit the study at any time (without providing a reason and without any consequence). Anonymity was assured as the e-mail addresses participants used to sign-up with were not linked to individual data. The 169 participants who signed-up were aged between 16 and 22 years ( $M=19.65 \Phi ; 1.5) .70 f$ these participants, 110 (65\%) were female, and 59 (35\%) were male. All were sexually active, heterosexual, and not in a relationship (where STI reducing safer-sex behavior plays a diminished role as compared to increased contraceptive measures, see, e.g., Bauman \& Berman, 2005; Manlove, Ryan, \& Franzetta, 2004; Manlove \& Terry-Humen, 2007) at the time of the study. In exchange for their participation, all participants received $€ 25$,- after completing the study.

\section{Procedure}

This two-part study was conducted entirely online, and procedures were explained in detail to the participant on a web page prior to the study. For this part of the study, participants were randomly assigned either to the sexually-aroused condition or to the nonaroused condition. After finishing the first part of the study, participants were thanked and reminded that in two weeks they would receive an e-mail containing a link to the second part of the study. This second part consisted of participants being assigned to the other condition - non-aroused if they had been assigned to the sexually aroused 
condition in the first part of the study - or sexually-aroused if they had previously been assigned to the non-aroused condition.

The first part of the study started with a short demographic questionnaire (assessing age, sexual orientation - heterosexual, homosexual, bisexual, undecided - and relationship status - single, in a relationship), followed by a 15-minute video clip (sexual or neutral, depending on the experimental condition), and the first assessment of sexual arousal. Participants then completed a unipolar Single Category Implicit Association Test (SC-IAT, see Karpinski \& Steinman, 2006) concerning the three behaviors of interest. The order of the SC-IATS was counterbalanced. This was followed by a 5-minute video clip (again, sexual or neutral, depending on the respective experimental condition) and the second assessment of sexual arousal. Participants then completed the letter span task to assess working memory capacity, followed by another 5 -minute video clip (again, depending on condition) and the third assessment of sexual arousal. Next, two blocks of questionnaires measured - in counterbalanced order - RAA variables (intention, attitude, social norms, perceived behavioral control, and past behavior related to the three behaviors of interest), life expectations, lifestyle inventory, and risk perception. In between the two blocks of questionnaires, the last 5-minute video was shown (sexual or neutral, depending on condition), after which their level of sexual arousal was assessed for the last time. The second part of the study was conducted two weeks after the first part and consisted of the same measures as the first part (with the exception of the short demographic questionnaire), but with participants now assigned to the other condition.

\section{Measures}

\section{Implicit associations}

The predictive validity of the Implicit Association Test (IAT; Greenwald, McGhee, \& Schwartz, 1998) has repeatedly been challenged (e.g., Jaccard \& Blanton, 2007; Rothermund \& Wentura, 2001) as well as confirmed (e.g., Horcajo, Rubio, Aguado, Hernández, \& Márquez, 2014). However, due to its robustness and reliability, it has become a widely used technique to measure relative strength of implicit association between two concepts, for instance, anxiety vs. calmness (Nosek, Greenwald, \& Banaji, 2007). These implicit associations are measured indirectly during a computerized categorization task, without asking people to reflect and report on their motivations for a specific behavior. IATs are based on the principle that people find it easier to categorize stimuli together if those stimuli are strongly associated as compared to stimuli that are only weakly - or are not - associated. However, IATs are bipolar meaning that two concepts are always required. To overcome this restriction, a new variant, the unipolar Single Category IAT (SC-IAT) has been introduced (Karpinski \& Steinman, 2006) in which only one attribute category is presented at a time. Therefore, a SC-IAT usually consists of three blocks (instead of five as in IATs) in which stimuli representing only one target category share a key with an evaluative concept (e.g., positive words). The other key is then used 
to respond to the opposite evaluative concept stimuli (e.g., negative words). A singlecategory implicit evaluation score can then be computed by comparing response latencies between blocks where the target category is paired with positive stimuli and blocks where the target category is paired with negative stimuli. Because IATs would create an artificial bipolarity in the assessment process of the targeted behaviors (assuming, for example, that there is an opposite concept to condom use), we used SC-IATs to assess participants' implicit attitudes toward the three protective behaviors of interest.

One SC-IAT measured implicit attitudes toward condom use with a new sex partner, one measured implicit attitudes towards asking a new sex partner about his/her sexual health status, and one measured implicit attitudes towards using less risky sex practices during first-time sex with a new sex partner. After the first SC-IAT had been completed, participants were allowed to take a break. Stimulus words for using condoms, asking a new sex partner about his/her sexual health status, and refraining from risky sex practices with a new sex partner were selected based on a pilot study in which 20 noun-verb pairs (e.g., use condoms) per target behavior were presented to 20 undergraduate students. For each noun-verb pair they were asked to rate (on a 7-point Likert-scale) how much this noun-verb pair described the target behavior (ranging from [1] "not at all" to [7] "very much"). Finally, seven noun-verb pairs per target behavior with a minimum score of five were used as stimulus material (see Table 1). Positive attribute words were: happiness, harmony, joy, and safety, whereas negative attribute words were: grief, hatred, harm, and stress.

Before the respective SC-IAT, and to account for possible individual differences in concept representation, each participant was asked to select four out of seven of these noun-verb pairs that best represented the respective target behavior. These four nounverb pairs were then used in the SC-IAT for that particular participant. The affective label words were always presented at the top of the screen, appropriately positioned on the left or the right side of the screen, depending on the required response (see Greenwald, McGhee, \& Schwartz, 1998). Each SC-IAT consisted of three blocks that came in a fixed order (see Table 2). 
Table 1. Word pairs per target behavior out of which participants were asked to choose four per respective SCIAT. The English translation of these word pairs is not always accurate, as some colloquial expressions cannot be literally translated from German without losing the original meaning.

\begin{tabular}{|c|c|c|}
\hline & German original & English translation \\
\hline \multirow[t]{7}{*}{ Condom SC-IAT } & Kondom gebrauchen & to use a condom \\
\hline & Präservativ gebrauchen & to use a preservative \\
\hline & Pariser gebrauchen & to use a Johnny \\
\hline & Verhüterli gebrauchen & to use a sheath \\
\hline & Gummi gebrauchen & to use a rubber \\
\hline & Lümmeltüte gebrauchen & to use a raincoat \\
\hline & Präser gebrauchen & to use a prophylactic \\
\hline \multirow[t]{7}{*}{ Asking SC-IAT } & Gesundheit abfragen & to inquire about health \\
\hline & Geschlechtskrankheiten abfragen & to inquire about sexually transmitted diseases \\
\hline & Gesundheitsrisiken nachfragen & to ask about health risks \\
\hline & STIs nachfragen & to ask about STIs \\
\hline & Gesundheitsstatus abfragen & to inquire about health status \\
\hline & Gesundheitszustand nachfragen & to ask about health status \\
\hline & Gesundheitsstörungen nachfragen & to ask about health problems \\
\hline \multirow[t]{7}{*}{ Practices SC-IAT } & Sexpraktiken anpassen & to adapt sex practices \\
\hline & Stellungen anpassen & to adapt sex positions \\
\hline & Stellungen aussuchen & to choose sex positions \\
\hline & Sexpraktik aussuchen & to choose sex practices \\
\hline & Stellung angeben & to specify sex position \\
\hline & Sextechnik wechseln & to change sex technique \\
\hline & Stellung wechseln & To change sex position \\
\hline
\end{tabular}

Table 2. Schematic overview of the block sequence in the Single Category-Implicit Association Tests (SC-IATS) measuring implicit attitudes toward condom use with a new sex partner (Condom SC-IAT), measuring implicit attitudes towards asking a new sex partner about his/her sexual health status (Asking SC-IAT), and measuring implicit attitudes towards performing less risky sex practices during first-time sex with a new sex partner (Practices SC-IAT).

\begin{tabular}{|c|c|c|c|c|c|c|}
\hline \multirow[b]{2}{*}{ Condom SC-IAT } & \multicolumn{2}{|c|}{$\begin{array}{l}\text { Block } 1 \\
\text { Attribute discrimination }\end{array}$} & \multicolumn{2}{|c|}{$\begin{array}{l}\text { Block } 2 \\
\text { Combination }\end{array}$} & \multicolumn{2}{|c|}{$\begin{array}{l}\text { Block } 3 \\
\text { Reversed combination }\end{array}$} \\
\hline & positive & negative & positive & $\begin{array}{l}\text { negative } \\
\text { condom word }\end{array}$ & $\begin{array}{l}\text { positive } \\
\text { condom word }\end{array}$ & negative \\
\hline Asking SC-IAT & positive & negative & positive & $\begin{array}{l}\text { negative } \\
\text { health word }\end{array}$ & $\begin{array}{l}\text { positive } \\
\text { health word }\end{array}$ & negative \\
\hline Practices SC-IAT & positive & negative & positive & $\begin{array}{l}\text { negative } \\
\text { practices word }\end{array}$ & $\begin{array}{l}\text { positive } \\
\text { practices word }\end{array}$ & negative \\
\hline
\end{tabular}

During block 1, the attribute discrimination phase, participants were instructed to press the left key if the stimulus word that comes up in the middle of their computer screen is a positive word (e.g., happiness) and press the right key if the stimulus word is negative (e.g., grief). In block 2, the combination phase, participants were instructed to press the 
left key if the stimulus word is a positive word and press the right key if the stimulus word is a negative word or a safer-sex behavior related noun-verb pair (e.g., use condom). In block 3 , the reversed combination phase, participants were instructed to press the left key if the stimulus word is a positive word or a safer-sex behavior related word and press the right key if the stimulus word is a negative word. The assignment of positive, negative, and safer-sex behavior related noun-verb pairs to the left or right side of the screen was fully counterbalanced. The IAT tasks were programmed in Inquisit 2.0 (Millisecond Software, USA). We chose D600 scores as the main outcome measure for the IAT (Greenwald, Nosek, \& Banaji, 2003). This algorithm includes all trials (including the attribute discrimination phase) and gives an error penalty of $600 \mathrm{~ms}$ to the reaction times on erroneous responses. The outcome is then divided by a personalized standard deviation of the combination phases so that the measure is not influenced by differences in response speed between participants. Higher D600 scores reflect a stronger association between sexual arousal and safer-sex behavior related noun-verb pairs.

\section{Working memory}

Participants completed a letter span task consisting of 30 trials (adapted from Klingberg, Forssberg, \& Westerberg, 2002) in which several letters were presented one at a time in a circle on the computer screen. After the last letter had disappeared, one of the positions in this circle was indicated, and participants were required to enter the corresponding letter using the keyboard. In the training condition, task difficulty was automatically adjusted on a trial-by-trial basis. The length of the sequences increased and decreased according to participants' performance, starting with a sequence of three letters. When participants correctly reproduced the sequence on two consecutive trials, one item was added to the sequence of the next trial whereas when participants did not correctly reproduce the sequence on two consecutive trials, the sequence in the next trial contained one item fewer.

\section{Explicit cognitions}

Scores on items that were included to assess the same variable were averaged into one measure in cases where internal consistency was sufficient (Cronbach's alpha $>.60$ or Pearson $r>.30$ with two items). Items were recoded such that higher scores reflect more agreement with the statement.

RAA measures. Participants' intention, attitude, subjective norms, and perceived behavioral control towards the three safer-sex behaviors (i.e., to use condoms, ask about the current sexual health status, and refrain from risky sex practices with a new sex partner) were each assessed by two items using 7-point Likert scales. Participants' past behavior related to condom use, asking about the current sexual health status, and refraining from risky sex practices with a new sex partner were each assessed by one question.

Risk perception. To assess young adults' risk perception across different domains, we used a shorter version of the Domain-Specific Risk-Taking (DOSPERT) Scale (Weber, Blais, 
\& Betz, 2002) that applies to a broad range of ages, cultures, and educational levels (Blais $\&$ Weber, 2006). We adapted this scale in order to be able to measure risk perception across different levels of sexual arousal in four subdomains of life: ethical (e.g., having sex with a boy/girl who is in a relationship), safety (e.g., engaging in unprotected sex), social (e.g., speaking one's mind about an unpopular issue in a meeting), and recreational (e.g., bungee jumping off a tall bridge). All items measured the respondents' gut-level assessment of how risky a specific behavior is. For each item participants were asked to rate the risk level of a specific behavior on a 7-point Likert-scale (ranging from [1] "not at all

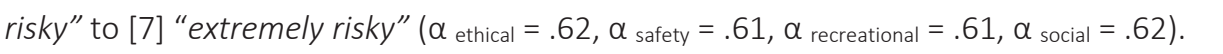

Life expectations. We adapted the life expectations' pool by Fischhoff et al. (2000) to assess whether young adults report different positive life expectations (e.g., having a 3year Bachelor degree by the time they turn 30) or negative life expectations (e.g., being the victim of a violent crime at least once in the next year) depending on the level of sexual arousal. Ten items assessed positive and negative expectations young adults have toward life events, such as earning a university degree or becoming the victim of a crime (adapted from Fischhoff et al., 2000). For each item, participants were asked to rate (on a 7-point Likert-scale) the likelihood that they would experience a specific situation (rang-

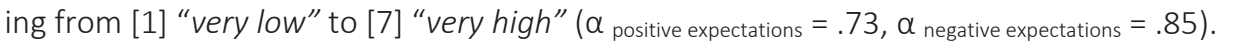

Lifestyle inventory. Health sustaining behavior covers multiple dimensions. We adapted the health behavior lifestyle inventory published by Forstmann, Burgmer, and Mussweiler (2012) to assess whether health-sustaining behaviors that are not directly related to risk-taking behavior would be influenced by the level of sexual arousal. Dimensions covered were eating habits (e.g., "I limit the amount of fat I eat"), health care prevention (e.g., "I go to the dentist at least once a year"), health care reaction - meaning strategies adopted after a health problem emerged - (e.g., "When I have a cold, I go to the doctor"), and personal hygiene (e.g., "I always wash my hands after I go to the restroom").

Twenty items assessed participants' behavior in four different life areas: eating, health care prevention, health care reaction (strategies adopted after a health problem occurred), and personal hygiene (adapted from Forstmann, Burgmer, \& Mussweiler, 2012). For each item participants were asked to rate (on a 7-point Likert-scale) how regularly they perform a specific behavior (ranging from [1] "never" to [7] "always" ( $\alpha$ eating $=$ .74 , plevention $=.61$, regaction $=.64$, holgiene $=.63$ ).

\section{Films to induce sexual arousal}

Films were shown at four different points in the study. One fifteen-minute clip was shown after a short demographic questionnaire at the beginning of the study, the second clip was shown after the last SC-IAT measurement, the third after the letter span task, and the fourth after administration of the first block of self-report questionnaires. Neutral 
films were taken from a documentary and showed nature footage (including audio) from Asian and African landscapes.

As audiovisual depiction of intercourse has been shown to elicit greater sexual arousal, especially in women (Chivers, Seto, Lalumiere, Laan, \& Grimbos, 2010), we chose erotic clips to induce sexual arousal. These clips showed sexual intercourse where male and female genital areas were blurred using the video processing software Premiere Pro CC (Adobe, USA). All erotic clips were standardized regarding different sexual scenes, i.e., oral sex and vaginal intercourse. None of the films showed sexual violence.

\section{Measurement of sexual arousal}

The level of sexual arousal was assessed after the presentation of each erotic video clip in the sexually aroused condition, as well as after each nature documentary in the nonaroused condition. Participants indicated on a visual analog scale (VAS) the level of their sexual arousal along a continuous line between " 0 ", representing "not at all sexually aroused" and "100" representing "extremely sexually aroused."

\section{Data analysis}

Data were analyzed using the predictive analytic computer software SPSS, version 24.0 (Statistical Product and Service Solutions, IBM, New York). First, a repeated-measures analysis of variance (ANOVA) was used to identify possible order and gender effects emerging from the random allocation of participants to either the aroused or the nonaroused condition. Following a descriptive analysis, a repeated-measures ANOVA was used to check the manipulation of sexual arousal. We expected to find a statistically significant difference between the sexual arousal conditions across the five measures of sexual arousal. SC-IAT D600 scores were analyzed with a multivariate analysis of variance (MANOVA) to identify a statistically significant difference between arousal conditions on the combined D600 scores of all three behaviors. When a significant multivariate effect was found, univariate analyses were conducted for each behavior. Paired-samples t-tests were then performed to detect statistically significant differences between sexual arousal conditions on the self-report measures and the working memory scores. Lastly, regression weights for the SC-IATs and for the RAA variables attitude, social norms, and perceived behavioral control (as well as their covariance) on intention towards each of the three behaviors of interest were calculated and modeled using multivariate linear regression analyses. To identify a possible statistically significant difference between the predictive power of the RAA variables on intention for each of the three protective behaviors depending on sexual arousal, path coefficients of the model were tested by comparing for each behavior - the constrained model (all paths being equal) with the respective unconstrained model. 


\section{Results}

A repeated-measures ANOVA did not reveal a significant interaction between condition order (either as a first or second condition) and sexual arousal on the outcome measures; all $F^{\prime} s<3.420$ and all $p^{\prime} s>$.066. We therefore excluded order from our analysis. The same analyses were repeated for gender $x$ sexual arousal interactions, but again no significant interactions were found; all $F^{\prime} s<0.605$ and all $p^{\prime} s>.612$.

\section{Induced sexual arousal}

Mean sexual arousal scores were higher in the sexually aroused trials ( $M=3 \quad 9.9 S B)$;

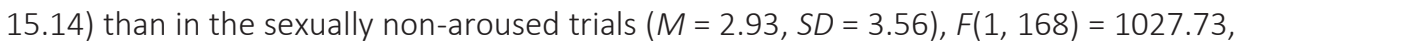
$p<.001,2$, p. 86 . No significant interaction of sexual arousal condition with time was found, $F^{\prime} s<1.95, p^{\prime} s>.12$, R'p $<.03$.

\section{SC-IAT}

A MANOVA revealed a statistically significant multivariate difference between the arousal

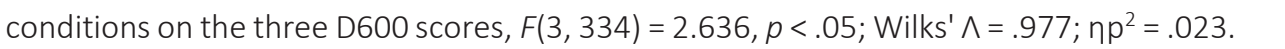
Subsequent univariate ANOVAs showed that the D600 scores for condom use and less risky sex practices did not differ significantly between the non-aroused state $(M=0.20$, $S D=0.3 \quad B 1=0.19 D,=0.3$, Respectively) and the sexually aroused state $(M=0.16 D$,

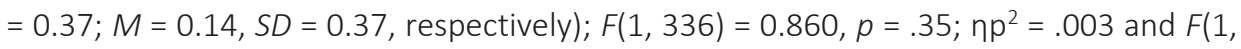
$336)=1.9 .16 ; h^{2}=006$, respectively. In contrast, for asking a new sex partner about his/her sexual health status, the D600 score was significantly higher in the sexually aroused state $\left(M=0.13 D_{1}=0.34\right)$ than in the manoused state $\left(M=0.0 \quad 6 D_{1}=\begin{array}{lll}0 & 0.3\end{array}\right)$,

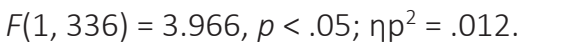

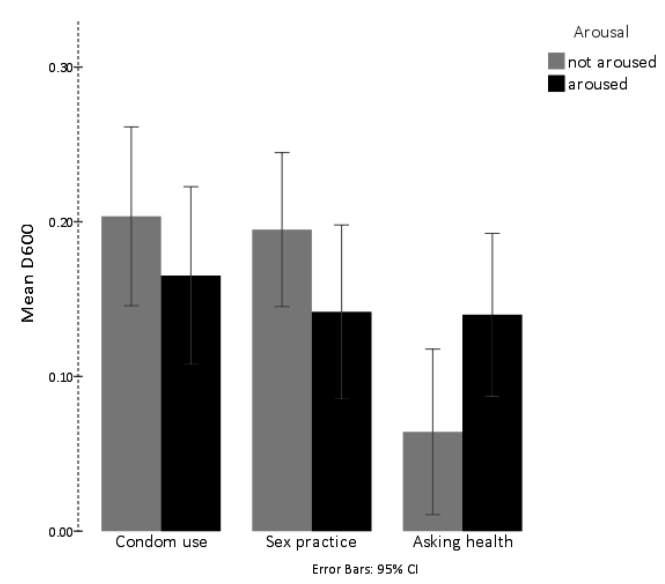

Figure 1. D600 scores for condom use, less risky sex practices, and asking a new sex partner about his/her sexual health status by the state of sexual arousal. 


\section{Self-report questionnaires}

Paired-samples t-tests showed significant effects of sexual arousal on the measures of positive life expectations, $t\left(\begin{array}{lll}1 & 6 & 8\end{array}\right)=8 . \$<.081, d=.64$, and negative life expectations, $t\left(\begin{array}{lll}1 & 6 & 8\end{array}\right)=7 . \$ 8.00,1, d=.60$. Higher scores were found on botthimensions of life expectation in the sexually arousal condition as compared to the non-aroused condition (see Table 3). Moreover, significant effects of arousal were found on the explicit attitude towards condom use, $t(168-2.775, p<.05, d=-.21$, and asking a new sex partner about his/her sexual health status, $t\left(\begin{array}{lll}1 & 6 & 8\end{array}\right)=2.75 .85, d=.17$. Attitudes for condom use were significantly more positive in a sexually non-aroused state than in a sexually aroused state, whereas attitude scores for asking a new sex partner about his/her sexual health status were significantly higher in a sexually aroused state than in a non-aroused state. On the other measures, no significant differences between the arousal conditions were found (see Table 3).

Table 3. Mean (M), standard deviation (SD), and Paired Samples Test results for questionnaire items in a sexually non-aroused and a sexually aroused state. $\mathrm{PBC}=$ perceived behavioral control.

\begin{tabular}{|c|c|c|c|c|c|c|}
\hline \multirow[t]{2}{*}{ Items } & \multicolumn{2}{|c|}{ Not aroused } & \multicolumn{2}{|c|}{ Aroused } & \multicolumn{2}{|c|}{ Paired Samples Test } \\
\hline & M & $S D$ & M & $S D$ & $t$ & $p$ \\
\hline Positive life expectations & 18.70 & 7.40 & 23.91 & 3.59 & 8.433 & $<.001$ \\
\hline Negative life expectations & 23.40 & 8.32 & 28.98 & 4.57 & 7.879 & $<.001$ \\
\hline Lifestyle eating & 22.46 & 3.27 & 22.44 & 3.82 & -0.076 & .940 \\
\hline Lifestyle prevention & 18.17 & 4.45 & 18.67 & 4.59 & 1.639 & .103 \\
\hline Lifestyle reaction & 13.80 & 3.49 & 14.14 & 3.91 & 1.170 & .244 \\
\hline Lifestyle hygiene & 24.26 & 4.06 & 23.93 & 4.27 & -0.999 & .319 \\
\hline Risk perception ethical & 22.66 & 3.56 & 23.02 & 3.75 & 1.309 & .192 \\
\hline Risk perception social & 16.34 & 3.47 & 16.37 & 3.69 & 0.100 & .921 \\
\hline Risk perception recreational & 26.86 & 4.55 & 26.47 & 4.99 & -1.193 & .235 \\
\hline Risk perception safety & 26.62 & 3.37 & 26.27 & 3.22 & -1.189 & .236 \\
\hline Attitudes condom use & 12.24 & 2.56 & 11.77 & 2.76 & -2.775 & .006 \\
\hline Attitudes sex practices & 10.15 & 2.94 & 9.88 & 3.27 & 1.164 & .246 \\
\hline Attitudes asking health & 8.82 & 2.85 & 9.25 & 2.92 & 2.258 & .025 \\
\hline Social norms condom use & 11.28 & 2.61 & 11.16 & 2.65 & -0.615 & .539 \\
\hline Social norms sex practices & 9.41 & 2.88 & 9.65 & 2.96 & 0.979 & .329 \\
\hline Social norms asking health & 8.70 & 3.03 & 9.08 & 2.91 & 1.835 & .068 \\
\hline PBC condom use & 6.24 & 1.34 & 6.11 & 1.36 & -0.992 & .323 \\
\hline PBC sex practices & 5.41 & 1.60 & 5.36 & 1.67 & -0.327 & .744 \\
\hline PBC asking health & 5.03 & 1.74 & 5.15 & 1.61 & 0.877 & .382 \\
\hline Intention condom use & 8.21 & 1.50 & 8.28 & 1.45 & 0.523 & .602 \\
\hline Intention sex practice & 7.98 & 1.88 & 8.05 & 1.48 & 0.371 & .711 \\
\hline Intention asking health & 8.21 & 1.65 & 8.08 & 1.39 & -0.772 & .441 \\
\hline
\end{tabular}




\section{Working memory}

Participants had less working memory capacity when in an aroused state $(M=8.7 \quad 3 D,-5$ 5.80) as opposed to a non-aroused state $(M=9.38 D,=5.39)$. However, this difference did not reach significance, $t\left(\begin{array}{lll}1 & 6 & 8\end{array}-1\right)=498, p=.13 \quad \mathbb{d}=-.11$.

\section{RAA model}

Figure 2 shows the path model for both the sexually non-aroused and the sexually aroused state according to the RAA. All three behavioral intentions were significantly predicted by their respective attitude, norms, and perceived behavioral control items in both the sexually non-aroused and the sexually aroused state (see Table 4 for the path coefficients of predictor variables on the outcome variable and Table 5 for the covariance of the predictor variables).

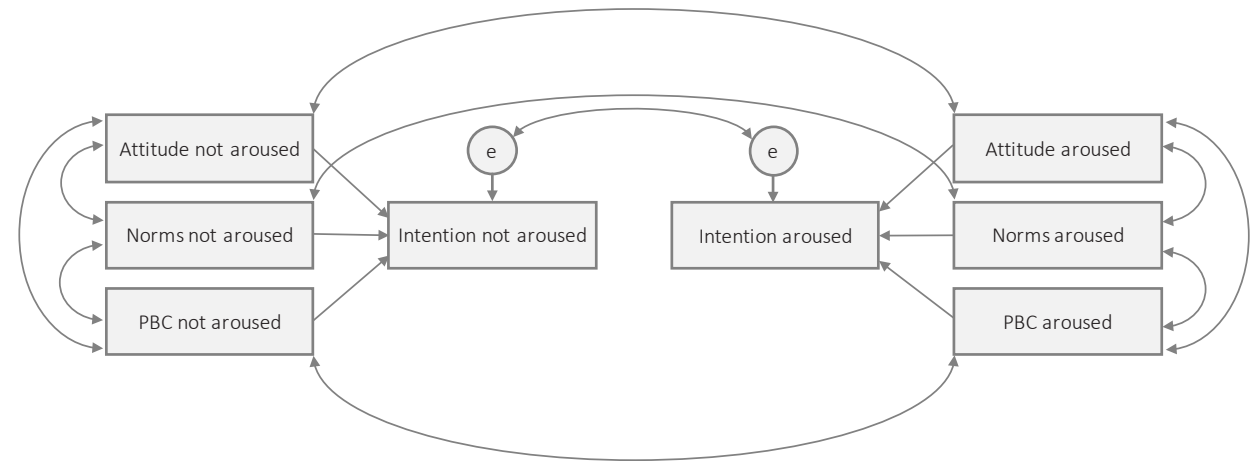

Figure 2. Model adapted from the Reasoned Action Approach to test measurement invariance across state of sexual arousal for attitude, norms, and perceived behavioral control (PBC) on the intention to use condoms, ask a new sex partner about his/her sexual health status, and refrain from risky sex practices (e = error terms).

We then tested the measurement invariance of the model across sexual arousal. However, measurement invariance was not rejected for each of the three protective behaviors, namely condom use: $\Delta \chi^{2}=1.26 d \bar{z}=, 3 p=.73$; sexulabalth question: $\Delta \chi^{2}=1.99$, $d f=3 p=.57$; sexual practic $A \chi^{2}=0.90 d \oplus=, 3 p=.82$.

Furthermore, we used hierarchical multiple regression to explore whether implicit attitudes added to the prediction of the intention to perform the three protective behaviors. The variance inflation factor (VIF) for these variables remained below two so that no multicollinearity was detected (a VIF greater than 10 indicates multicollinearity, see Bowerman \& O'Connell, 1990; Myers, 1990). The addition of weight to RAA variables to the prediction of intentions was statistically not significant for all three protective behaviors. Table 6 shows mean, SD, $\mathrm{R}^{2}$ and $\mathrm{R}^{2}$ change, and centralized regression coefficients (betas). 
Table 4. Standardized regression weights (B), standard errors (S.E.), critical ratio (C.R.), and statistical significance $(p)$ for Reasoned Action Approach variables predicting intention to use condoms with a new sex partner, to ask a new sex partner about his/her sexual health status, and to refrain from risky sex practices during firsttime sex with a new partner, PBC = perceived behavioral control.

\begin{tabular}{|c|c|c|c|c|c|c|c|c|c|}
\hline \multirow[t]{2}{*}{ Factors } & & \multicolumn{4}{|c|}{ Not sexually aroused } & \multicolumn{4}{|c|}{ Sexually aroused } \\
\hline & & B & S.E. & C.R. & $p$ & b & S.E. & C.R. & $p$ \\
\hline \multirow[t]{3}{*}{ Intention practice } & $\leftarrow$ Attitude practice & 0.311 & 0.082 & 4.377 & $<.001$ & 0.419 & 0.076 & 5.628 & $<.001$ \\
\hline & $\leftarrow$ Norms practice & 0.211 & 0.084 & 2.92 & $<.05$ & 0.231 & 0.073 & 3.308 & $<.001$ \\
\hline & $\leftarrow$ PBC practice & 0.215 & 0.095 & 2.819 & $<.05$ & 0.153 & 0.072 & 2.304 & $<.05$ \\
\hline \multirow[t]{3}{*}{ Intention condoms } & $\leftarrow$ Attitude condoms & s0.452 & 0.071 & 6.408 & $<.001$ & 0.404 & 0.062 & 5.753 & $<.001$ \\
\hline & $\leftarrow$ Norms condoms & 0.127 & 0.079 & 1.879 & $<.05$ & 0.202 & 0.062 & 2.902 & $<.05$ \\
\hline & $\leftarrow \mathrm{PBC}$ condoms & 0.135 & 0.081 & 1.936 & $<.05$ & 0.148 & 0.075 & 2.135 & $<.05$ \\
\hline \multirow[t]{3}{*}{ Intention asking } & $\leftarrow$ Attitude asking & 0.355 & 0.08 & 5.107 & $<.001$ & 0.363 & 0.069 & 5.321 & $<.001$ \\
\hline & $\leftarrow$ Norm asking & 0.251 & 0.07 & 3.954 & $<.001$ & 0.157 & 0.075 & 2.444 & $<.05$ \\
\hline & $\leftarrow$ PBC asking & 0.218 & 0.082 & 3.157 & $<.05$ & 0.294 & 0.083 & 4.157 & $<.001$ \\
\hline
\end{tabular}

Table 5. Standardized regression weights (B), standard errors (S.E.), critical ratio (C.R.), and statistical significance $(p)$ for the covariance of Reasoned Action Approach variables predicting intention to use condoms with a new sex partner, to ask a new sex partner about his/her sexual health status, and to refrain from risky sex practices during first-time sex with a new partner, $\mathrm{PBC}=$ perceived behavioral control.

\begin{tabular}{|c|c|c|c|c|c|c|c|c|c|}
\hline \multirow[t]{2}{*}{ Covariates } & & \multicolumn{4}{|c|}{ Not sexually aroused } & \multicolumn{4}{|c|}{ Sexually aroused } \\
\hline & & $B$ & S.E. & C.R. & $p$ & B & S.E. & C.R. & $p$ \\
\hline Norm practice & $\leftrightarrow$ PBC practice & 0.47 & 0.232 & 5.518 & $<.001$ & 0.367 & 0.238 & 4.464 & $<.001$ \\
\hline Attitude practice & $\leftrightarrow$ Norms practice & 0.328 & 0.239 & 4.034 & $<.001$ & 0.56 & 0.274 & 6.333 & $<.001$ \\
\hline Attitude practice & $\leftrightarrow$ PBC practice & 0.442 & 0.233 & 5.238 & $<.001$ & 0.488 & 0.256 & 5.689 & $<.001$ \\
\hline Attitude condoms & $\leftrightarrow \mathrm{PBC}$ condoms & 0.389 & 0.172 & 4.7 & $<.001$ & 0.39 & 0.189 & 4.708 & $<.001$ \\
\hline Norm condoms & $\leftrightarrow \mathrm{PBC}$ condoms & 0.282 & 0.143 & 3.523 & $<.001$ & 0.376 & 0.184 & 4.565 & $<.001$ \\
\hline Attitude condoms & $\leftrightarrow$ Norm condoms & 0.325 & 0.167 & 4.009 & $<.001$ & 0.402 & 0.228 & 4.84 & $<.001$ \\
\hline Norm asking & $\leftrightarrow$ PBC asking & 0.225 & 0.259 & 2.849 & $<.05$ & 0.367 & 0.216 & 4.47 & $<.001$ \\
\hline Attitude asking & $\leftrightarrow$ Norm asking & 0.256 & 0.268 & 3.21 & $<.05$ & 0.268 & 0.241 & 3.357 & $<.001$ \\
\hline Attitude asking & $\leftrightarrow$ PBC asking & 0.459 & 0.265 & 5.407 & $<.001$ & 0.488 & 0.26 & 5.688 & $<.001$ \\
\hline
\end{tabular}

Table 6. Means (M), standard deviations (SD), standard errors (S.E.), standardized regression weights $(B), R$ squares $\left(R^{2}\right)$, R square changes ( $R^{2}$ change), and the significance level of $F$ changes (Sig. $F$ change) of a hierarchical multiple regression showing relationships between implicit attitudes and intentions.

\begin{tabular}{lllllllll}
\hline Intention & Implicit attitudes & Mean & SD & S.E. & $B$ & $R^{2}$ & $R^{2}$ change Sig. F Change \\
\hline Practice & Not aroused & .19 & .328 & .390 & .163 & .333 & .001 & .676 \\
& Sexually aroused & .14 & .370 & .291 & .289 & .451 & .003 & .322 \\
\multirow{2}{*}{ Condoms } & Not aroused & .20 & .381 & .262 & .236 & .336 & .003 & .368 \\
& Sexually aroused & .17 & .378 & .244 & -.096 & .362 & .001 & .695 \\
\multirow{2}{*}{ Asking } & Not aroused & .06 & .352 & .364 & .284 & .380 & .002 & .436 \\
& Sexually aroused & .14 & .346 & .339 & .030 & .412 & .001 & .623 \\
\hline
\end{tabular}




\section{Discussion}

In this study, we examined the effect of sexual arousal on implicit associations and selfreported explicit attitudes, social norms, perceived behavioral control and intentions related to three safer-sex behaviors with a new sex partner (using condoms, asking about the sexual health status of a new partner, and refraining from risky sex practices) as well as on self-reported life expectations, lifestyle decisions, and domain-specific risk perceptions. Furthermore, we investigated the effect of sexual arousal on working memory as a proxy of executive functions. We then tested if the predictive power of the main determinants of behavioral intention advocated by the RAA model (Fishbein \& Ajzen, 2010) - namely attitude, social norms, and perceived behavioral control - would be altered depending on the level of sexual arousal.

We were able to induce a moderate level of sexual arousal that was independent of participants' sex and remained stable during the time of the study. Participants had significantly more positive implicit and explicit attitudes towards asking a new sex partner about his/her current sexual health status when in a state of sexual arousal. More positive attitudes towards asking such a delicate question (that might put an imminent mating experience at risk) suggest greater disinhibition during sexual arousal as compared to a non-arousal. This finding extends results of previous studies (e.g., Imhoff \& Schmidt, 2014; Skakoon-Sparling, Cramer, \& Shuper, 2015) which have shown that engaging in uncommon and unprotected sexual behavior is more likely to occur in acute states of sexual arousal. Our results indicate that sexual arousal might also facilitate some protective health behaviors - such as asking a partner about possible STIs - as individuals tend to be more disinhibited in an aroused as compared to a non-aroused state. In contrast, explicit, but not implicit, attitudes towards condom use were significantly more positive in a nonaroused state as compared to a sexually aroused state. These results corroborate Ariely and Loewenstein's (2006) findings that sexual arousal decreases the relative importance of protection against STIs. As suggested by disinhibition theory, external factors such as drugs or alcohol can encourage participation in high-risk sexual activities (e.g., Snipes \& Benotsch, 2013). In summary, our findings suggest that greater disinhibition during sexual arousal could lead to risky health behaviors, such as condom non-use, but also to protective health behaviors, such as asking a new sex partner about his/her current sexual health status. However, in order to corroborate this protective effect, we suggest that replications of our study are needed.

Our data also indicate that sexual arousal had an amplifying effect on positive as well as negative life expectations. In this way, positive life expectations were perceived more positively during sexual arousal than in a non-aroused state. Similarly, negative life expectations were reported as being more negative while sexually aroused compared to a sexually non-aroused state. While it has previously been shown that an emotion-oriented attentional focus has regulatory effects on subjective sexual arousal (Both, Laan, \& Everaerd, 2011), our results indicate that sexual arousal might amplify such an attentional 
focus. The influence of sexual arousal on the perception of life events could result from an increase in salience of mating goals (Hill \& Durante, 2011a). As life expectations are likely to be influenced by dispositional factors (e.g., skills) and situational factors (e.g., cooperation of others), they could influence control beliefs in the RAA model. We suggest that as control beliefs influence the perceived behavioral control towards a specific behavior - here safer-sex behavior - amplified positive life expectations could possibly strengthen perceived behavioral control, whereas amplified negative life expectations could diminish perceived behavioral control.

We did not find an effect of sexual arousal on lifestyle inventory items, risk perception, social norms, perceived behavioral control, or intentions towards the three safersex behaviors of interest. Furthermore, there was no significant difference in working memory capacity depending on the level of sexual arousal. Our data indicate that sexual arousal does not influence a broad range of explicit variables. Instead, we found only an effect on attitudes and life expectations.

As for the predictive power of the RAA, the central components (attitudes, social norms, and perceived behavioral control) towards the three behaviors of interest did not show a statistically significant difference in terms of sexual arousal, indicating that the model predicts behavioral intentions equally well independent of the level of sexual arousal.

This study has some limitations. The current study was conducted online which allowed participants to choose an adequate location to ensure their privacy and to reduce inhibitions. This, of course, reduced our ability to control for the participants' careful and conscientious implementation of the study. Therefore, we cannot exclude the possibility that some participants may not have followed all instructions - or may have been distracted by parallel activities. Second, our experimental setup did not allow us to use physiological methods to measure participants' level of sexual arousal. Instead, we relied on self-report measures that have been shown to only weakly (Chivers et al., 2010) or even negatively (Grauvogl et al., 2015) correlate with genital measures of sexual arousal, such as vaginal pulse amplitudes. In fact, self-reported sexual arousal may reflect more of a higher-order conscious appraisal of sexual cues, and therefore be more open to personal influences such as the cultural meaning of particular sexual cues, as compared to genital measurements that may represent faster and more unconscious information processing (Janssen, Everaerd, Spiering, \& Janssen, 2000; Janssen, Prause, \& Geer, 2007). Third, it was not possible to observe actual behavior, so that we were only able to measure participants' predictions of their behavior under different levels of sexual arousal (Bouffard, 2002; Loewenstein, Nagin, \& Paternoster, 1997). In fact, we only used the RAA variables that directly predict behavioral intention, without measuring the actual behavior. Such measurements could have provided valuable information about the effects of additional predictors of behavior (such as perceived behavioral control) at different levels of sexual arousal. Fourth, even though our data indicate that greater disinhibition during sexual arousal could lead to health protective behavior (asking a new sex partner about his/her 
current sexual health status), without replication, we can only hypothesize that this protective behavior occurs by lowering the barriers and enabling individuals to ask potentially embarrassing questions. Lastly, the mean level of sexual arousal measured in our study was around 40\%. Participants were thus only mildly sexually aroused. Outcome measures in conditions of high sexual arousal might have been different. Therefore, our results might not be applicable to highly aroused states, which are likely to occur at the moment of sexual decision making.

\section{Conclusion}

Our results suggest that behavioral disinhibition due to sexual arousal can support - but also reduce - health-sustaining sexual behavior. More research into this dual role of disinhibition is needed to better understand how this factor should be taken into consideration - especially in combination with alcohol intake - in safer-sex education programs. Furthermore, our data showed that explicit positive and negative life expectations were amplified during sexual arousal. Understanding whether life expectations influence control beliefs which then, as proposed by the RAA, influence perceived behavioral control, could constitute the next step towards developing more effective safer-sex education programs. 



\section{Chapter Five}

\section{ERPs Reveal Disengagement Processes During}

Condom Use Embarrassment in Intentionbehavior Inconsistent Young Adults

Based on:

Brüll, P., Kessels, L.T.E., Dirkson, A., Repetto, L., Ruiter, R.A.C. (2018). ERPs Reveal

Disengagement processes during condom use embarrassment in intention-behavior inconsistent young adults. Archives of Sexual behavior. 


\section{Abstract}

The use of barrier protections such as condoms has consistently been reported to reduce the acquisition of sexually transmitted infections. However, it has also been reported that the association between condom use intentions and behavior is, at best, often weak. Furthermore, embarrassment associated with purchasing condoms and negotiating their use has been shown to negatively impact the frequency of condom use. Using electroencephalography to analyze P300 event-related potential components known to measure early attention allocation, we examined electrophysiological evidence of early attention disengagement for embarrassing health information. Forty young adults -34 females and six males-participated in an adapted version of Posner's visual cueing paradigm. All were high in intention to use condoms, but half were intention-behavior consistent, and half were intention-behavior inconsistent. Compared to intention-behavior consistent participants, those with intention-behavior inconsistency showed a reduced P300 component when attending to a visual target opposite to the field in which embarrassing self-relevant health information was presented, indicating more efficient early attention disengagement from such embarrassing health information. In conclusion, our electrophysiological data suggest that high intention alone may be not sufficient to predict adolescents' condom use behavior. 


\section{Introduction}

The use of barrier protections such as condoms has been consistently shown to reduce the acquisition of STIs (e.g., Weller \& Davis, 2002). Furthermore, a relationship between the intention to use condoms and actual condom use behavior has been demonstrated in multiple studies (see the meta-analysis by Albarracín et al., 20 0. Neyertheless, it has also repeatedly been reported that the association between condom use intention and actual behavior is often weak (Bauman et al., 2007; Fridlund et al., 2014; Sheeran \& Orbell, 1998; Smith \& de Visser, 2004; Turchik \& Gidycz, 2012). In fact, behavioral intention appears to account for just $30 \%$ of the variance in actual behavior (Armitage \& Conner, 2001; Sheeran, 2002a). This inconsistency between intention and behavior (e.g., when people with positive intentions to perform a behavior fail to act on their intentions) is referred to as "the intention-behavior gap" (Sheeran, 2002a). Given that intention-behavior gaps have been observed in relation to various different health-promoting behaviors, including physical activity, weight loss, and illicit drug use (Sheeran, 2002a; Webb \& Sheeran, 2006), it seems important to understand the mechanisms that determine the strength of the link between intention and behavior.

Assertive condom negotiation strategies (e.g., withholding sex and direct request for condom use) have been identified as the most important factors associated with increased condom use in both women and men (French \& Holland, 2013). The use of these strategies can increase perceived self-efficacy in terms of condom negotiations; this, together with a low level of fear regarding the negotiation of condom use, and good communication with sex partners about condom use, has been shown to predict consistent condom use (Crosby, DiClemente, et al., 2013). However, it has also been reported that the embarrassment associated with purchasing condoms-and negotiating their usenegatively impacts the frequency of condom use (Moore et al., 2008; Moore, Dahl, Gorn, \& Weinberg, 2006). Embarrassment can be defined as a distinct emotion which threatens an individual's social identity during an interaction (Keltner \& Buswell, 1997). Furthermore, embarrassment has been linked to the belief that others might detect a personal flaw (Sabini, Garvey, \& Hall, 2016), and has recently been shown to predict avoidance of sexual health sustaining behavior such as genital examinations and discussions of sexuality with health care professionals (McCambridge \& Consedine, 2014). It is known that health-related information that is perceived to be self-relevant, but for which the recommended health action is perceived to be difficult to implement, is likely to induce defensive reactions (Peters, Ruiter, \& Kok, 2012). This can be explained by Festinger's (1957) dissonance theory, and Kunda's (1990) motivated reasoning approach. To date, defensive reactions related to embarrassment have not been studied extensively (Consedine, Krivoshekova, \& Harris, 2007). Studies investigating the amount of attention that is allocated to health information (e.g., Brown \& Locker, 2009; Liberman \& Chaiken, 1992; Mogg, Bradley, De Bono, \& Painter, 1997) have found that individuals who are most at risk react defensively to intimidating health messages by avoiding those messages or 
attending away from them. Discussing condom use with a new sex partner clearly entails a potentially embarrassing situation (Moore et al., 2006), which may, via avoidance, result in weakening-or even a disruption - of the intention-behavior link.

Electroencephalography (EEG) is a continuous recording of brain activity with EventRelated Potentials (ERPs) as time-locked measures of averaged cortical electrical activity representing a distinct phase of cortical processing. The P300 ERPs have a maximum positive peak around 300 ms after stimulus onset (Bentin, Mouchetant-Rostaing, Giard, Echallier, \& Pernier, 1999) and are shorter over frontal areas and longer over parietal areas (Conroy \& Polich, 2007). They show larger amplitudes when a target stimulus is attended to as compared to when little or no attention is allocated to the target stimulus (Kok, 1997). The P300 ERP component is therefore suitable for detecting early attention allocation (Böcker, Baas, Leon Kenemans, \& Verbaten, 2004; Johnson, 1993; Polich, 2007), especially for self-relevant stimuli (Gray, Ambady, Lowenthal, \& Deldin, 2004; Patel \& Azzam, 2005). As shown by Kessels et al. (2010), EEG can be used to investigate defensive responses to (threatening) health messages. They demonstrated that smokers for whom the information was self-relevant disengaged more efficiently (and thus used fewer attentional resources) from high-threat smoking pictures than from low-threat smoking pictures on invalid trials (where the target stimulus was presented on the opposite side of the screen as the cue). This was reflected in lower P300 amplitudes for smokers for high- as opposed to low-threat trials. For non-smokers, for whom the information was not self-relevant, no significant difference in P300 activity was found. Reduced P300 amplitudes have also been shown in women with hypoactive sexual desire disorder during the viewing of low-intensity sexual films as compared to high-intensity sexual films (Vardi et al., 2006). Prause, Steele, Staley, and Sabatinelli (2015) investigated the late positive potential (LPP), an ERP with an early time window of 300-600 ms reflecting facilitated attention to emotional stimuli (Schupp et al., 2000; Schupp, Junghöfer, Weike, \& Hamm, 2004). They found that the LPP was reduced in response to less explicit sexual images for participants who reported fewer intercourse partners in the last year as compared to those who reported more sexual intercourse partners (and who showed similar LPP amplitudes to both more and less explicit sexual images). Furthermore, Krug, Plihal, Fehm, and Born (2000) reported an influence of the menstrual cycle on LPPs. This was demonstrated by an increased amplitude to sexual stimuli during the ovulatory phase, suggesting a greater valence of sexual stimuli during a phase that is marked by increased sexual desire.

In the present study, we examined whether negotiation regarding condom use induces an early attention disengagement process related to embarrassment. We expected that pictures depicting condom negotiation would induce embarrassment. Furthermore, we predicted that individuals with high intention to use condoms but with inconsistent (past) condom use behavior (intention-behavior inconsistent individuals), as compared to those with high intention to use condoms and consistent (past) condom use behavior (intention-behavior consistent individuals), would attempt to reduce their feeling of 
dissonance by disengaging attention from those pictures. Adopting the experimental paradigm used by Kessels et al. (2010), we therefore expected that intention-behavior inconsistent participants would make use of fewer attentional resources to detect visual targets presented in opposite areas of the visual field in which the uncomfortable cue was presented (invalid trials), as compared to individuals for whom the information was less self-relevant (i.e., participants with high intention and consistent (past) condom use behavior). In this attention cueing paradigm, reactions to targets in the invalid trials thus reflect disengagement processes.

As the use of attentional resources is reflected in both the amplitude of the P300 ERP and in reaction times as measured by a specific key press following stimulus presentation (e.g., Ito, Larsen, Smith, \& Cacioppo, 1998), we expected participants with intention-behavior inconsistency to show lower P300 amplitudes and faster reaction times in invalid and embarrassing trials than intention-behavior consistent participants. Targets in valid trials (where the target stimulus is presented on the same side of the screen as the cue) were expected to induce similar patterns of results for behavior consistent and behavior inconsistent participants because these trials reflect mere attention-capturing processes.

\section{Methods}

\section{Participants}

A total of 245 undergraduate university students were recruited from various public places at the university as well as from social media sites. They completed a short online questionnaire that asked about demographics (age, sex), sexual identity (heterosexual, homosexual, bisexual, undecided), and current relationship status (in a romantic relationship, not in a romantic relationship). Next, their intention to use condoms during first time sex with a new sex partner was assessed ("I intend to always use condoms when I have sex with a new sex partner for the first time", using a 7-point Likert-scale, ranging from [1] "unlikely" to [7] "likely"). Finally, past condom use behavior was assessed ("Last time I had sex with a new sex partner for the first time, I used condoms," using a 7-point Likert-scale, ranging from [1] "false" to [7] "true"). Scores on items that assessed the same variable were averaged into one measure in cases where internal consistency was sufficient (Cronbach's alpha $\geq 0.70$ with two items). Only participants who reported a high intention ( 6 or 7 on the 7-point Likert-scale) to use condoms during first time intercourse with a new partner were selected. Within this high intention group, those respondents who reported having used a condom the last time they had intercourse with a new sex partner ( 6 or 7 on the 7-point Likert-scale) were asked to participate in the study. The first 20 respondents who agreed to participate formed the intention-behavior consistent group. Similarly, the first 20 respondents from the high intention group who reported not to have used a condom during their last first-time intercourse with a new 
sex partner (1 or 2 on the 7-point Likert-scale) comprised the intention-behavior inconsistent group. In total, 40 young adults (34 females, 6 males) aged between 18 and 25 years $(M=21$ year $\$ D=1.4$ Were thus selected for participation in the EEG study according to their survey results. All participants gave their written informed consent as required by the Institutional Review Board and received a gift voucher of $€ 25$ after their participation. All participants were right-handed, had a normal or corrected-to-normal vision, were heterosexual, and were sexually active but not involved in a romantic relationship at the time of the experiment.
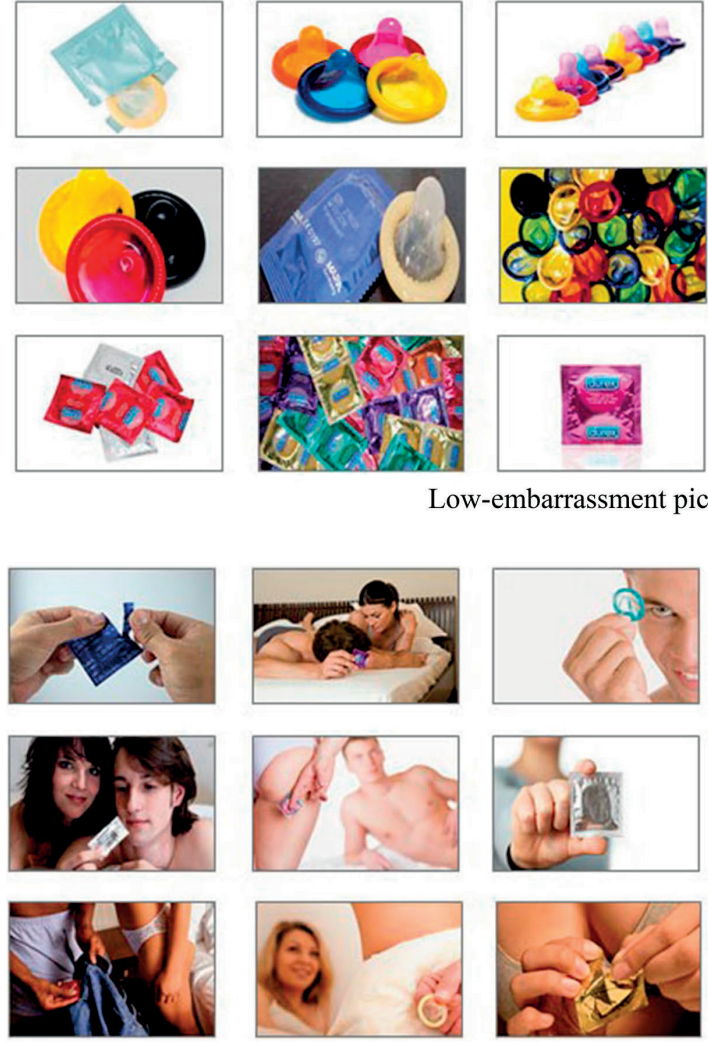

High-embarrassment pictures
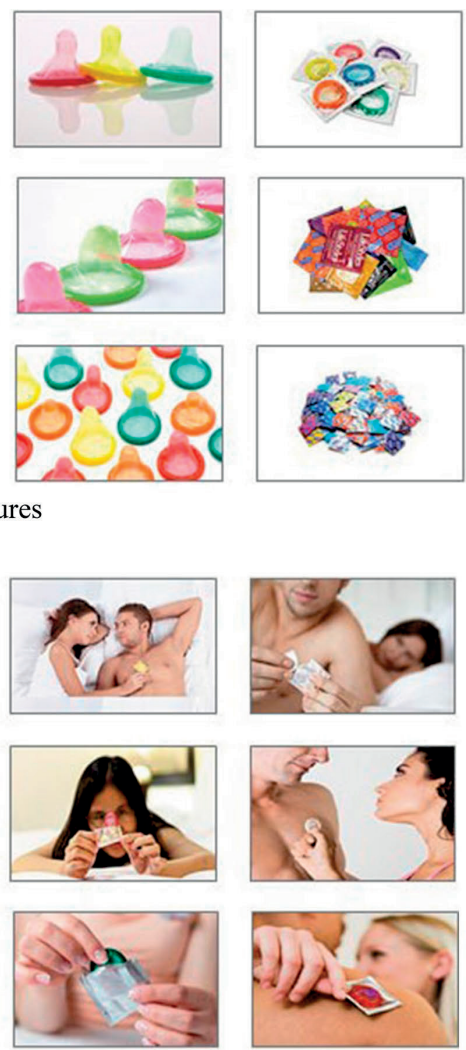

Figure 1. Fifteen color low-embarrassment pictures showing condoms and 15 high-embarrassment color pictures showing condoms that were used as cues in the cueing task.

\section{Materials}

In total, 30 color pictures of condoms -either high or low in embarrassment -were used as cues in the cueing task. The low-embarrassment pictures showed only condoms while high-embarrassment pictures showed individuals holding a condom in their hands (see Fig. 1). The selection of these pictures was based on a pilot study in which 100 condomrelated pictures were presented to 50 undergraduate students. For each picture, they 
were asked to rate their perceived level of discomfort on a 7-point Likert-scale (ranging from [1] "not at all uncomfortable" to [7] "extremely uncomfortable") and embarrassment (ranging from [1] "not at all embarrassed" to [7] "extremely embarrassed"), Cronbach's alpha $=0.71$. Finally, 15 highly embarrassing eminglarrassment) pictures with a minimum score of five and 15 only slightly embarrassing (low-embarrassment) pictures with a maximum score of three were selected and used as stimulus material. During the EEG-experiment, the level of embarrassment (high vs. low) and the validity of the trials (valid vs. invalid) were varied as within-subjects factors. Behavioral consistency in relation to intention (intention-behavior consistent vs. intention-behavior inconsistent) was included as a between-subjects factor.

\section{Procedure}

For the cueing paradigm, pictures (255 x 170 pixels) were presented at the left or right side of the midpoint of a 17-inch computer screen with a distance of $8 \mathrm{~cm}$. Two horizontal dots (..) or two vertical dots (:) were used as target stimuli. In each trial, a fixation cross was presented for 1,400 ms in the middle of the computer screen, followed by a highembarrassment or a low-embarrassment cue for 500 ms (see Fig. 2). After a second fixation period of $200 \mathrm{~ms}$, a target stimulus was presented for another $200 \mathrm{~ms}$, either on the same side of the computer screen as the cue (valid trial: $82 \%$ of trials) or on the opposite side of the computer screen (invalid trial: $18 \%$ of trials). There were 1,320 trials in total (cue and target), comprising 1,080 valid trials and 240 invalid trials. Cues in the valid condition correctly indicated the position of the upcoming target whereas cues in the invalid condition appeared on the side opposite to the subsequent target. These cue-target combinations were randomly presented within each of the 33 experimental runs. The intertrial interval was 2,300 ms. After each block of 40 trials, participants were allowed to take a short break.

Valid Trial

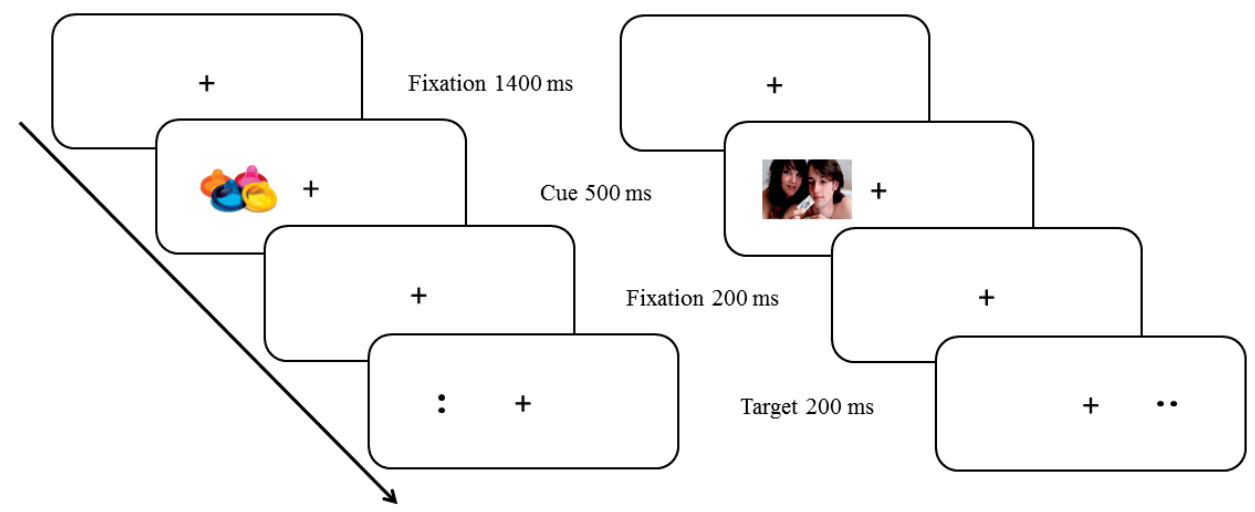

Figure 2. Experimental procedure showing an example of low-embarrassment pictures (valid trial) and highembarrassment pictures (invalid trials). 
Participants sat approximatively $80 \mathrm{~cm}$ from a computer screen on which the experimental stimuli were displayed. Before the start of the experiment, a priming screen with all of the high-embarrassment pictures was shown to the participants. They were asked to look at these pictures for 1 minute and to imagine themselves being in that very situation, meaning a situation in which they had to negotiate condom use or take the initiative to use condoms. They were then once more instructed to imagine themselves being in such a situation for the time in which the pictures were presented on the screen. Participants were further instructed to keep their eyes fixated on the fixation cross and to press the left button as quickly as possible when two horizontal dots were presented and to press the right button as quickly as possible when two vertical dots were presented. They were also told that the probability of a valid trial was larger than the probability of an invalid trial. A practice block lasting 2 minutes allowed participants to familiarize themselves with keeping their gaze fixed on the fixation cross while responding correctly to the targets.

After the cueing task, participants were asked to evaluate the perceived level of embarrassment related to each picture on a 7-point Likert-scale (ranging from [1] "not at all embarrassing" to [7] "extremely embarrassing"). The entire experiment lasted approximately two hours.

\section{Measures}

\section{Reaction times and errors}

Button-press responses were measured from target onset with errors consisting of mistakes (e.g., the participant pressed right for horizontal dots and left for vertical dots), misses (no button press), and reaction times faster than 120 ms and slower than 1000 ms.

\section{EEG and ERPS}

The EEG was recorded from 21 scalp sites with $\mathrm{Ag} / \mathrm{AgCl}$ sintered electrodes, following the $10 / 20$ system. Electrodes were mounted in an electrode cap, and the EEG recordings were referenced online to the left mastoid and re-referenced offline to the average of the left and right mastoids by subtracting one-half the amplitude of the right mastoid to the left mastoid recording. Horizontal eye movements were recorded with a bipolar montage of electrodes, placed on the right and left external canthus and vertical eye movements and eye blinks were recorded on the upper and lower orbital ridge of the left eye. The electro-oculogram allowed that trials contaminated with eye movements were later rejected offline. All electrode impedances were kept below $5 \mathrm{k} \Omega$ and signals were digitized at a $250 \mathrm{~Hz}$ sampling rate using a 32 channel BrainVision (Brain Products, Munich, Germany) amplifier. A bandpass filter of 0.05 to $30 \mathrm{~Hz}$ was used to filter the continuous EEG offline. From the continuous EEG signal, epochs of 1,500 ms were obtained for ERP analysis, starting at $100 \mathrm{~ms}$ pre-cue onset (serving as a baseline) and ending $600 \mathrm{~ms}$ after 
target offset. Epochs containing artifacts beyond $-1 \quad 0 \quad 0$ and $100 \mu \mu$ were removed before analysis. In total, $19 \%$ of the analyzed trials as well as the entire data sets of four participants could not be used due to excessive movement artifacts. This left a total number of 18 participants per condition: 16 females and two males aged between 18 and 24 years $(M=20$ year $\$ D=1.5)$ in the intentiebehavior consistent group, and 15 females and three males aged between 18 and 23 years $(M=20$ year $\$=1.6)$ in the intention behavior inconsistent group. There was no significant age difference between both groups, $t\left(\begin{array}{ll}1 & 8\end{array}\right)=0 . A=5.3,1$. The EEG sequences for each combination of embarrassment, validity, and electrode site for each participant were averaged to ERPs. Finally, a grand average of these individual ERP data was calculated per experimental condition and electrode site for both intention-behavior consistent and inconsistent groups.

\section{Analyses}

Mixed analyses of variance (ANOVAs) with embarrassment (high vs. low) and validity (valid vs. invalid) as the within-subject factors and intention-behavior consistency (consistent vs. inconsistent) as the between-subjects factor were used to analyze reaction times and errors. ERP analyses were restricted to midline electrodes $\mathrm{Fz}, \mathrm{FCz}, \mathrm{Cz}, \mathrm{CPz}$, and $\mathrm{Pz}$ because attention effects are generally largest for these midline electrodes (Johnson, 1993; Polich, 2007). To control for an unequal distribution of valid and invalid trials, separate embarrassment (high vs. low) x electrode ( $\mathrm{Fz}, \mathrm{FCz}, \mathrm{Cz}, \mathrm{CPz}, \mathrm{Pz}$ ) x consistency (consistent vs. inconsistent) mixed measures ANOVAs were performed for reactions to the valid and the invalid targets respectively. To control for sphericity violations in the ANOVAs, probability values with Greenhouse-Geisser correction for $F$ tests with more than 1 degree of freedom in the numerator are reported, and the reported estimates of the effect size are the partial eta squared $\left(\eta_{p}{ }^{2}\right)$ for the analyses of variance.

\section{Results}

\section{Error Analysis}

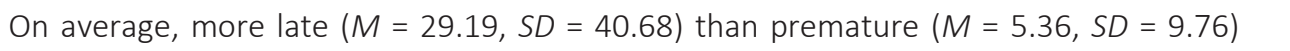
responses per participant were excluded, $t(35)=4$. 5 . 8 .001. As shown in Table 1, compared to intention-behavior consistent participants, intention-behavior inconsistent participants tended to make more errors across conditions, but these differences failed to reach statistical significance. No main effects or interactions involving the factors embarrassment, validity, and behavioral consistency were found ( $\left.p^{\prime} s>.175\right)$. 
Table 1. Behavioral performance measures as a function of trial validity, embarrassment, and past behavior consistency.

\begin{tabular}{|c|c|c|c|c|c|c|c|c|}
\hline \multirow[b]{3}{*}{ Variable } & \multicolumn{4}{|l|}{ Valid } & \multicolumn{4}{|l|}{ Invalid } \\
\hline & \multicolumn{2}{|c|}{ High emb. } & \multicolumn{2}{|c|}{ Low emb. } & \multicolumn{2}{|c|}{ High emb. } & \multicolumn{2}{|c|}{ Low emb. } \\
\hline & M & SD & M & SD & $\mathrm{M}$ & SD & M & SD \\
\hline \multicolumn{9}{|c|}{ Reaction time (ms) } \\
\hline I-B consistent & 540.17 & 72.21 & 549.56 & 75.69 & 564.00 & 74.43 & 571.83 & 77.83 \\
\hline I-B inconsistent & 535.78 & 61.96 & 535.89 & 59.40 & 552.67 & 57.30 & 556.11 & 62.42 \\
\hline \multicolumn{9}{|l|}{ Errors (\%) } \\
\hline I-B consistent & 6.22 & 28.87 & 5.74 & 24.57 & 7.06 & 6.08 & 6.62 & 5.05 \\
\hline I-B inconsistent & 7.68 & 22.84 & 7.21 & 22.52 & 8.68 & 27.06 & 9.03 & 29.88 \\
\hline
\end{tabular}

\section{Reaction Times}

Mean reaction times (in $\mathrm{ms}$ ) to the target stimuli (horizontal and vertical dots) are also shown in Table 1. As expected, independent of intention-behavior consistency, reaction

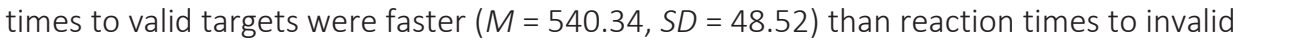

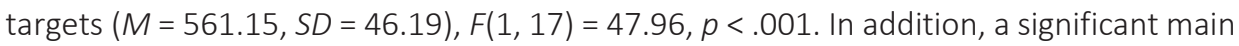
effect for embarrassment showed that reaction times to high-embarrassment targets ( $M$ $\left.=\begin{array}{lll}548 . & 8 D 5=, 4 \\ 6 & .69 & 9\end{array}\right)$ were faster than todowbarrassment targets $(M=5 \quad 5 \quad 3.3 D 4$, 47.55), $F(1,17)=6 p . \varnothing 0,5$. No further main effects or interaction effects were found, $F^{\prime} s<2.33, p^{\prime} s>.80$.

\section{ERP Analyses}

Visual inspection. Visual inspection of the ERP waves in reaction to the target stimuli showed the expected P300 effect on the midline electrodes Fz, FCz, Cz, CPz, and Pz (Table 2). The grand average ERP waveforms indicate that the expected $P 300$ effect for the invalid trials was most strongly concentrated at the frontal-central electrodes between 240 and $340 \mathrm{~ms}$ after target onset (Fig. 3). Based on this visual inspection, we concentrated our separate analyses on the $\mathrm{Fz}, \mathrm{FCz}$, and $\mathrm{Cz}$ electrodes.

P300. An overall mixed ANOVA for all five midline electrodes for the invalid trials showed a significant three-way interaction between electrodes, embarrassment, and intentionbehavior consistency, $F(4,31)=3 p 4<00,5 p \eta=.27$. There was a furthergnificant interaction effect between embarrassment and electrodes, $F\left(\begin{array}{lll}4,3 & 1\end{array}\right)=3 p K D, 5 p \eta=$ .28, and a significant main effect of electrodes, $F(4,31)=1$ Qp $3.060,1_{p}^{2}$, 7.58 
Table 2. Amplitudes $(\mu \mathrm{V})$ of the P3 00 effect føaction of trial validity, level of embarrassment, and behavioral consistency.

\begin{tabular}{|c|c|c|c|c|c|c|c|c|}
\hline \multirow[t]{3}{*}{ Electrode } & \multicolumn{4}{|l|}{ Valid } & \multicolumn{4}{|c|}{ Invalid } \\
\hline & \multicolumn{2}{|c|}{ High emb. } & \multicolumn{2}{|c|}{ Low emb. } & \multicolumn{2}{|c|}{ High emb. } & \multicolumn{2}{|c|}{ Low emb. } \\
\hline & $\mathrm{M}$ & SD & $\mathrm{M}$ & SD & $\mathrm{M}$ & SD & $\mathrm{M}$ & SD \\
\hline \multicolumn{9}{|l|}{$\mathrm{Fz}$} \\
\hline Consistent & 3.84 & 2.05 & 3.77 & 1.94 & 3.10 & 2.11 & 3.25 & 1.96 \\
\hline Inconsistent & 3.05 & 2.20 & 3.08 & 2.05 & 2.04 & 2.52 & 1.86 & 2.22 \\
\hline \multicolumn{9}{|l|}{$\mathrm{FCz}$} \\
\hline Consistent & 4.93 & 2.49 & 5.28 & 2.43 & 5.65 & 1.90 & 4.75 & 2.38 \\
\hline Inconsistent & 4.16 & 2.63 & 4.29 & 2.80 & 3.91 & 2.86 & 3.12 & 3.28 \\
\hline \multicolumn{9}{|l|}{$\mathrm{Cz}$} \\
\hline Consistent & 5.79 & 2.42 & 6.39 & 2.99 & 7.07 & 1.60 & 5.32 & 2.16 \\
\hline Inconsistent & 5.16 & 3.04 & 5.57 & 3.10 & 5.01 & 3.45 & 3.51 & 3.41 \\
\hline \multicolumn{9}{|l|}{$\mathrm{CPz}$} \\
\hline Consistent & 6.83 & 2.43 & 7.36 & 2.22 & 7.08 & 1.69 & 7.79 & 2.14 \\
\hline Inconsistent & 6.00 & 3.15 & 6.52 & 3.37 & 6.52 & 3.68 & 6.16 & 3.63 \\
\hline \multicolumn{9}{|l|}{$\mathrm{Pz}$} \\
\hline Consistent & 7.33 & 2.50 & 7.89 & 2.39 & 7.67 & 1.66 & 8.53 & 2.12 \\
\hline Inconsistent & 6.09 & 2.93 & 6.74 & 3.27 & 6.73 & 3.47 & 6.68 & 3.48 \\
\hline
\end{tabular}

More specific mixed ANOVAs for the invalid trials on the $\mathrm{Fz}, \mathrm{FCz}$, and $\mathrm{Cz}$ electrodes, where the P300 effect was most strongly concentrated, also showed a significant three-way interaction between embarrassment, intention-behavior consistency and electrodes, $F(2$, 33 ) = $6.0<1.05$ p $7=.26$, a significant main effect of embarrassment $(1,34)=5.94$, $p<.05 p_{p} \eta=.14$, and a significant main effect of electrode $5(2,3 \quad 3)=6$ op.5. $\$$. $\$ 01$, $\eta_{p}{ }^{2}=.78$. Mixed ANOVAs for the invalid trials on the Cland $\mathrm{Pz}$ electrodes revealed only a main effect of electrodes, $F(1,34)=7 p \ll \quad D, 5 p \eta=.17$.

Simple effect analyses for each level of embarrassment showed a significant interaction between intention-behavior consistency and electrodes for both high-embarrassment invalid trials, $F(2,33)=1 \Phi \leqslant 1030,1_{p}^{2}, 7.38$, and ftorw-embarrassment invalid trials, $F(2,33)=7 p \ll$ \$ $3,0 \quad 1_{p}^{2}$, 7.7 5In the high-embarrassment condition, P300 amplitudes at the three electrodes $\mathrm{Fz}, \mathrm{FCz}$, and $\mathrm{Cz}$ were significantly lower for intentionbehavior inconsistent participants as compared to intention-behavior consistent participants, $F^{\prime} s>10.26, p^{\prime} s<.003{ }_{p}^{2}{ }^{\prime \prime} \$>.23$. These effects were largest at electrode $\mathrm{Cz}$ and smallest at electrode Fz. P300 amplitudes in the low-embarrassment condition at all three electrodes were also significantly lower for intention-behavior inconsistent participants as compared to intention-behavior consistent participants, $F^{\prime} s>13.06, p^{\prime} s<.001$, $\eta_{p}{ }^{2 \prime} s>.27$. 


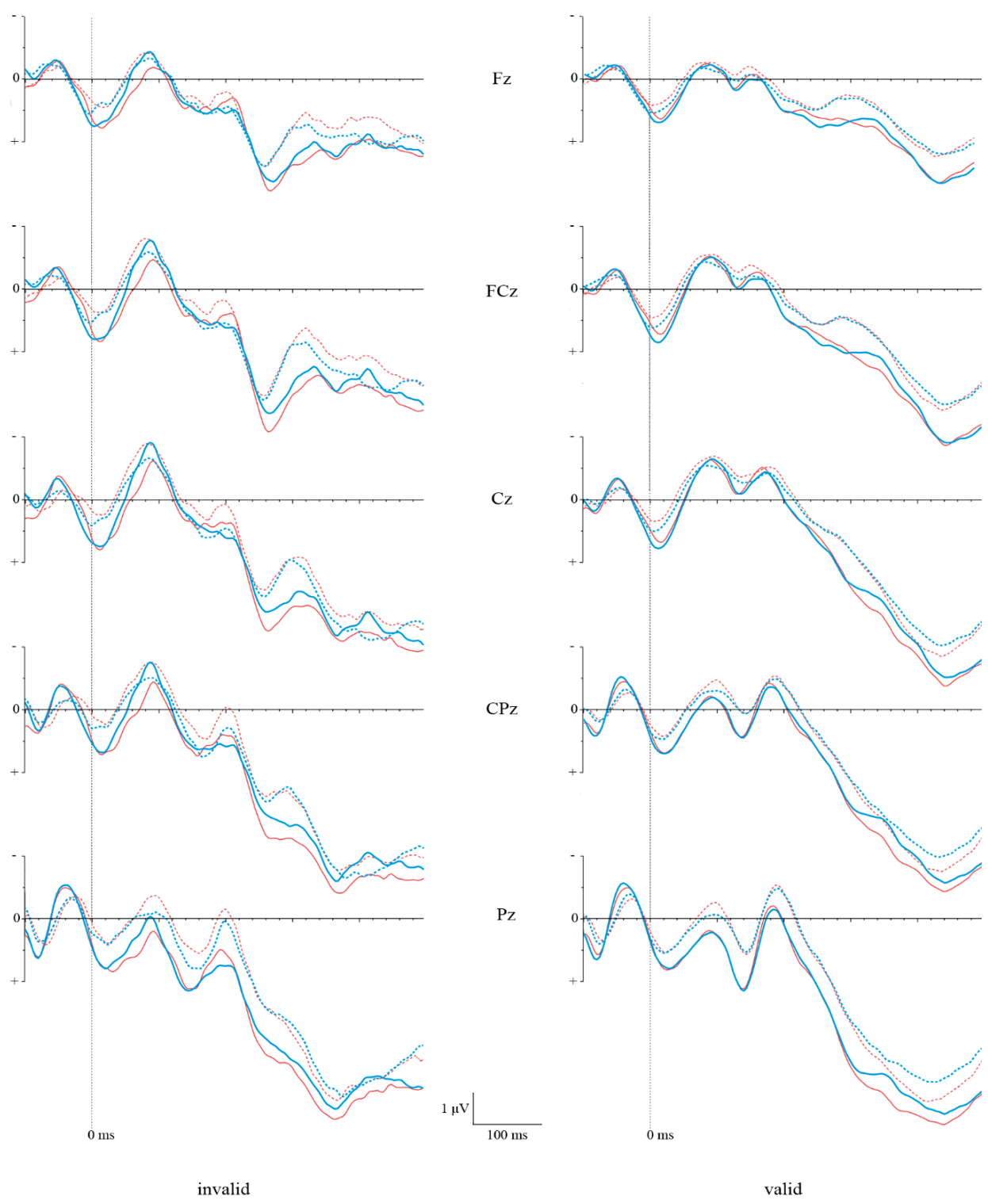

Figure 3. Grand average P300 event-related potentials (ERPs) for high- and low-embarrassment stimuli across intention-behavior consistent and inconsistent groups in invalid and valid trials: high embarrassment, intentionbehavior consistency (full red line); high embarrassment, intention-behavior inconsistency (dashed red line); low embarrassment, intention-behavior consistency (full blue line); low embarrassment, intention-behavior inconsistency (dashed blue line).

Separate analysis for each level of intention-behavior consistency for invalid trials showed a significant interaction between embarrassment and electrodes for both intention-behavior inconsistent participants, $F(2,16)=4$ p9<6.0, $5, p_{n} \eta=.3$, 6 and for intention- 
behavior consistent participants, $F(2,16)=7 p 6<10,055_{p}^{2}$, 月.4 8While intention-behavior inconsistent participants showed no significant difference in P300 amplitudes between high- and low-embarrassment trials across the three electrodes ( $F^{\prime} s<3.33, p^{\prime} s>$ 86 , 8 its < .16), intention-behavior consistent participants showed significantly lower P300 amplitudes for low-embarrassment trials as compared to high-embarrassment trials

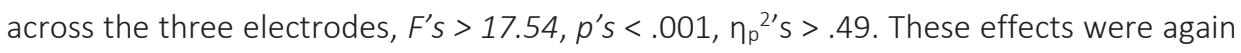
largest at electrode $\mathrm{Cz}$ and smallest at electrode $\mathrm{Fz}$.

The mixed ANOVA for all five midline electrodes for the valid trials did not show any interaction effects ( $F^{\prime} s<.86, p^{\prime} s>.22, \eta_{p}{ }^{2} s<.17$ ) but revealed a significant main effect of

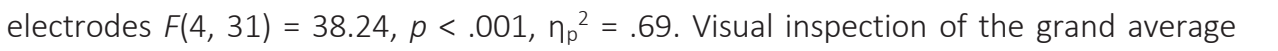
waveforms of the midline electrodes (Fig. 3) suggests larger amplitudes of the P300 effect for parietal electrodes as compared to frontal-central electrodes (see also Table 2).

\section{Self-Report Measures}

Intention-behavior inconsistent participants rated the embarrassment level of the highembarrassment pictures significantly higher $\left(M=\begin{array}{l}1.8 \\ \hline\end{array} \bar{D},=\begin{array}{lll}0.7 & 1\end{array}\right)$ than the embarrass-

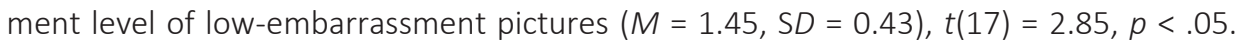
Furthermore, intention-behavior consistent participants also rated the level of embarrassment significantly higher for high-embarrassment $\left(M=\begin{array}{llll}1 & 8 & 3 D & =0 \\ 0 & .7 & 7\end{array}\right)$ versus tow embarrassment $(M=1.35 D=0.44)$ picturka, 7$)=3 . \beta$. 3.05 . However, there was no significant difference between intention-behavior consistent and intention-behavior inconsistent participants for the ratings of high-embarrassment pictures, $F\left(\begin{array}{ll}1 & 3 \\ 5\end{array}\right)=0.32$, $p=.85$, and of loembarrassment pictures, $F(1,35)=0.5 \beta=.47$.

\section{Discussion}

Using a visual selective attention paradigm, we found support for our hypothesis that young adults with intention-behavior inconsistency (as related to condom use) displayed more efficient disengagement from both high- and low-embarrassment pictures displaying condoms and condom use negotiations than intention-behavior consistent participants. In fact, for invalid trials, intention-behavior inconsistent participants had lower P300 peak amplitudes for both low- and high-embarrassment trials as compared to intention-behavior consistent participants. Interestingly, compared to intention-behavior consistent participants, participants with past condom use-related intention-behavior inconsistency showed a significantly decreased P300 amplitude in invalid trials in response to condom-related pictures, independent of the level of embarrassment. In fact, compared to intention-behavior consistent participants, intention-behavior inconsistent participants disengaged attention more efficiently from even slightly embarrassing (low embarrassment) pictures. This could be related to feelings of self-threat, in line with 
Festinger (1957) and Kunda (1990) who proposed that people want to reduce feelings of cognitive dissonance by altering the respective cognitive or behavioral elements. Intention-behavior inconsistent participants may do this by already attending away from even slightly embarrassing information.

We did not, therefore, find support for our prediction that intention-behavior inconsistent young adults disengaged attention more efficiently from pictures they rated as highly embarrassing (pictures showing condom use negotiations) than from pictures they rated as slightly embarrassing (pictures showing condoms only). Rather, we found that intention-behavior consistent young adults disengaged attention more efficiently from low-embarrassment pictures than from high-embarrassment pictures, as indicated by lower P300 peaks for low-embarrassment stimuli compared to high-embarrassment stimuli in invalid trials. This finding may be in line with previous research that showed higher P300 amplitudes for self-relevant stimuli compared to neutral stimuli (e.g., Gray et al., 2004). One could argue that for those individuals who act upon their health intentions, mere pictures of condoms are perceived as less self-relevant than pictures showing familiar condom negotiation situations, resulting in lower P300 amplitudes.

As indicated by our ERP results in invalid trials, attention disengagement can arise within 300 ms after stimulus presentation and reflects an early process of attention disengagement from embarrassing health information. Even if participants with inconsistent past condom use behavior explicitly reported their intention to use condoms during first time sex with a new partner, it was the embarrassing information related to condoms that may have caused the observed involuntary shift of spatial attention, referred to as attentional capture by Remington, Folk, and McLean (2001). These participants attended to stimulus properties relevant to their implicit behavioral goals, namely to avoid a potentially embarrassing situation. Such an involuntary attention shift as shown in our ERP results has been proposed to be contingent on the relationship between the eliciting event and the required task performance (Folk, Remington, \& Johnston, 1992). According to the central tenet of Edelmann's (1985) embarrassment theory, protective self-presentation can conflict with knowledge of social rules. Our study revealed the persistence of embarrassment in a group of intention-behavior inconsistent young adults and underscores the importance of directing coping strategies at overcoming this embarrassment. In fact, simply having good intentions to use condoms may not result in actual condom use (the intention may not be translated into behavior) if condoms and condom use are associated with potentially embarrassing situations. It follows, then, that embarrassment associated with condom use negotiation could represent a barrier to consistent condom use with a new sex partner among young adult populations.

However, embarrassing and control images may have differed on other dimensions besides embarrassment level, namely on erotic content and inclusion of people. It is possible that the attentional disengagement could have been due to differing responses to the higher level of erotic content in the embarrassing images. Erotic stimuli have been shown to involuntarily draw one's attention, just as visual orientation is drawn towards 
threat-related stimuli (Sennwald et al., 2016). This constitutes an alternative explanation for the higher P300 for high- versus low-embarrassment images within the intention-behavior consistent group. It may also explain the higher P300 and increased attentional engagement in the intention-behavior consistent group for the embarrassing images (if indeed they did find them more erotic than the intention-behavior inconsistent participants).

Alternatively, the inclusion of people only in the embarrassing images could have influenced attentional processing, as participants may respond differentially to social situations. For instance, individuals with social phobia and anxiety show difficulties with attentional disengagement from socially threatening social stimuli (Amir, Elias, Klumpp, \& Przeworski, 2003; Amir et al., 2010; Bantin, Stevens, Gerlach, \& Hermann, 2016; Beard, Sawyer, \& Hofmann, 2012; Heeren, Mogoase, Philippot, \& McNally, 2015). On the other hand, the majority of studies indicate that individuals with autism spectrum disorder have decreased visual attention to social stimuli compared to healthy individuals (Bellocchi, Henry, \& Baghdadli, 2017). While we are not suggesting that our participants fulfill any of these clinical criteria, these characteristics do exist on a spectrum within the general population. The possibility that higher levels of social avoidance may also have contributed to the intention-behavior inconsistency via a reduced ability to communicate about condom use is also worth noting.

Attention disengagement can be decreased by increasing the proportion of targets appearing at the location of the intended training bias stimuli in a dot-probe task (Lazarov \& Bar-Haim, 2016). It has been shown that such attention bias modification training (ABMT) aimed at directing attention towards stimuli can significantly increase attentional bias towards and consumption of healthy food (Kakoschke, Kemps, \& Tiggemann, 2014) and alcohol (Field \& Eastwood, 2005) in healthy participants. Furthermore, ABMT has been shown to be effective in reducing negative - or threat-related - attention bias by directing attention away from threat-relevant stimuli in healthy controls facing a stressful situation (Mogoaşe, David, \& Koster, 201 a\& well as in various patient groups, such as adolescents with depression (Lazarov \& Bar-Haim, 2016) or individuals with social phobia (Amir et al., 2010) or anxiety (Beard et al., 2012; Heeren et al., 2015).

Given these results, it may also be feasible to increase attention towards condomrelated stimuli, thereby decreasing attention disengagement, using ABMT. However, such interventions remain challenging because internet-based ABMT on personal computers has not been shown to have any significant effect (Boettcher et al., 2013; Boettcher, Berger, \& Renneberg, 2012; Carlbring et al., 2012; Neubauer et al., 2013). Alternatively, smartphones remain a potential medium, but more research is necessary. To our knowledge, only one study has used smartphones to deliver ABMT to individuals with social anxiety, resulting in a small effect on attention bias scores (Enock, Hofmann, \& McNally, 2014).

Our prediction that intention-behavior inconsistent participants would show faster reaction times than intention-behavior consistent participants in response to high- 
embarrassment pictures was not confirmed. One possible reason for this could be the large response speed variability in our sample (Van Damme \& Crombez, 2009). However, ERP waves showed significant differences between both groups in time windows that preceded response preparation, indicating that attention towards embarrassing health information is modulated at an early stage of cognitive processing. As expected, we found an effect of embarrassment across both groups as reflected in faster reaction times, and hence faster disengagement, in the highly embarrassing condition. Furthermore, an effect of validity was identified across both groups, indicating faster responses to valid as opposed to invalid trials. The latter finding is in line with Pollak and Tolley-Schell (2003), who showed faster reaction times in cueing tasks for valid as compared to invalid trials. However, unlike Pollak and Tolley-Schell, we did not find higher accuracy rates for valid over invalid trials. Indeed, in our study, intention-behavior consistent participants showed less accuracy in valid trials, independent of embarrassment, and no significant difference was found between valid and invalid trials for intention-behavior inconsistent participants. We can only speculate that intention-behavior consistent participants may have reduced their attention to the target due to the limited self-relevance of the previously ipsilateral presented cue whereas contralateral presented targets may have increased participants' attention due to their new position on the screen.

There are several possible limitations to our study. First, our sample consisted of 31 female and five male participants. While taking into account the finding that women's perceived relationship power control has not been found to be associated with safer-sex practices (Hahm, Lee, Rough, \& Strathdee, 2012), we nevertheless cannot exclude the possibility that a gender bias related to intention and past behavior measures influenced our results. Second, our study did not include a control group, so we cannot infer a causal link between embarrassment and condom use behavior. It is worth noting that we did not ask participants about their recent experiences with sex-related or condom-related negotiations, meaning that we cannot exclude the possibility that recent adverse experiences may have influenced our findings. Third, the cueing task consisted of low-embarrassment pictures that showed condoms only, while high-embarrassment pictures showed individuals holding a condom in their hands- thus inferring a situation involving condom use negotiation. Even though participants were asked to imagine themselves in that fictional situation - and thus imagine being involved in a negotiation about condom use - we cannot exclude the possibility of a response bias occurring related to the differing content (condoms versus condoms + individuals) of these pictures. Lastly, no-cue conditions are commonly used in behavioral studies using a cueing task. In a no-cue condition, target stimuli are presented without previous warning, allowing the investigation of an effect of validity on reaction times (e.g., Baayen \& Milin, 2010 ; Perchet \& García Larrea, 2000). The present study did not use such no-cue conditions. While using ERP measures, no-cue trials could act as a confounding variable because of the added factor of surprise due to unanticipated stimuli (Jonides \& Mack, 1984; Pollak \& Tolley-Schell, 2003). The fact that we did not include no-cue conditions means that we cannot 
determine whether the effect of validity we found for reaction times reflects a cost related to an invalid cue or a benefit related to a valid cue.

\section{Conclusion}

In conclusion, the present study showed that, compared to intention-behavior consistent young adults, those with intention-behavior inconsistency disengaged attention more efficiently from low- and high-embarrassment pictures related to condoms and condom use. This was independent of their high intentions to use condoms during their next firsttime sex with a new partner. Our findings suggest that a high level of intention alone may be insufficient to predict condom use behavior if its implementation is associated with feelings of embarrassment. Future research could address some of the limitations of this study. We recommend questioning subjects about the subjective eroticism of the images, in addition to assessing their general social skills. Another recommendation would be to utilize a more extensive set of control stimuli: condoms alone, people alone and condoms combined with people. 



\section{Chapter Six}

\section{Heroes of the Night - Promoting Three Safer-Sex Behaviors by Targeting Explicit and Implicit Behavioral Determinants in a Serious Game}

Based on:

Brüll, P., Hebecker, R. Wiers, R. W., Kok, G. \& Ruiter, R. A. C. (under review). Heroes of the Night - Promoting three safer-sex behaviors by targeting explicit and implicit behavioral determinants in a serious game. 


\section{Abstract}

Young people (aged 15-24 years) still remain at high risk of acquiring sexually transmitted infections, including HIV. In this study, we combined evaluative conditioning, taskrelevant response inhibition training, and working memory training in a newly developed serious game. We tested whether playing the game would strengthen explicit behavioral determinants (particularly behavioral intention) and implicit attitudes towards implementing three protective behaviors with a new sex partner: (1) using condoms, (2) asking about his or her partner's current sexual health status, and (3) refraining from risky sex practices. In total, 195 young people, aged between 18 and 24 years, were randomly assigned to 13 conditions of a serious game, consisting of different combinations of interventions and a varying number of gaming sessions. The results were mixed, as reflected partly in a strengthening of (mostly explicit) behavioral determinants and partly in a weakening of those variables, especially after three or five gaming sessions with all intervention components active. We suggest that, while the use of a serious game is a promising delivery tool, focusing on one behavior at a time over the course of several gaming sessions may well result in more favorable outcomes. 


\section{Introduction}

Even after more than 20 years of evidence-based behavioral interventions to reduce sexually transmitted infections (STIS), including infections with the human immunodeficiency virus (HIV), the number of people living with STIs and/or HIV remains high (Globerman et al., 2017). In particular, young people aged 15-24 years are at high risk of acquiring STIs (Centers for Disease Control and Prevention, 2017). In Western and Central Europe, the prevalence rate of new HIV infections in this age group remains fairly stable at $0.2 \%$ for females and $0.3 \%$ for males (Unaids, 2013). However, with a quarter of a billion young people acquiring a curable STI for the first time every year, this age group accounts for nearly half of all new curable STIs at a global level (World Health Organization, 2013). A great variety of approaches for improving young people's sexual decision-making have been tested, including abstinence-only and comprehensive education programs (Carter, 2012; Kirby, 2008; Kohler et al., 2008; Stanger-Hall \& Hall, 2011), curriculum-based programs (Kirby \& Laris, 2009; Kirby et al., 2007), sexual health promoting programs (Schaalma et al., 2004), the promotion of monogamy (Conley et al., 2015), parental engagement strategies (Centers for Disease Control and Prevention, 2014), and multipurpose prevention technologies (Fernández-Romero et al., 2015), so far with varying degrees of success (Globerman et al., 2017). What has become clear, however, is that the tailored development of programs for young people can succeed in lowering the high STI rate in this population (Catalano et al., 2012; World Health Organization, 2010), especially when delivered using digital technology such as internet, mobile phones, and gaming consoles (All et al., 2014).

In western industrialized countries, $93 \%$ of young people are online and $97 \%$ play video games (Kachur, R., Mesnick, J., Liddon, N., Kapsimalis, C., Habel, M., David-Ferdon et al., 2013). Such a massive online presence of young people suggests that an exploration of how to increase and sustain gamification of behavior-change interventions related to STI prevention may be fruitful (Enah et al., 2013). These commonly termed serious or educational games are interventions delivered online by computers or mobile devices. The aim of these games is to educate or promote behavior change while participation in these interventions remain enjoyable and intrinsically motivating (Graesser et al., 2009; Kato, 2010; Prensky, 2007). Even if some online interventions have successfully promoted behavior change (Hamari et al., 2014), a recent meta-analysis showed that educational games fail to induce a long-term effect on behavior if they are not tailored toward behavioral outcomes and if they fail to take sociodemographic information and behavioral change needs into account (DeSmet et al., 2014b). This kind of tailoring of computerbased interventions can lead to positive behavior outcomes, such as decreased calorie consumption (Starks, 2014), smoking cessation (El-Hilly et al., 2016), and especially increased protective sexual health behavior (Noar et al., 2009; Portnoy et al., 2008). Moreover, it has been suggested that involving the target population as informants (through feedback and input) or as co-designers (through direct involvement) from an 
early stage onwards - a process known as participatory design (PD) - may be associated with increased effectiveness as compared to interventions with no user involvement. However, in a recent meta-analysis, DeSmet et al. (2016) did not find increased effectiveness of games developed with PD. However, they did report that games developed with PD were significantly more effective when participation was focused on game dynamics rather than game aesthetics.

The present study adds a novel component to the increasing range of behaviors that have been targeted by serious games (for an overview, see Göbel, 2016). Using PD (Brüll, Ruiter, Wiers, \& Kok, 2016a), we combined evaluative conditioning with the training of executive functions (response inhibition and working memory). Our study comprised of several conditions, in which we varied the number of gaming sessions and the combination of blocks containing active and mock instances of evaluative conditioning and executive function training.

\section{Explicit and implicit determinants of behavior}

The Reasoned Action Approach (RAA) proposed by Fishbein and Ajzen (2010) is an explanatory model that can be used to identify determinants of intention to perform the three safer-sex behaviors of interest in this study. Emerging out of the Theory of Reasoned Action (TRA) (Fishbein \& Ajzen, 1975), and the Theory of Planned Behavior (TPB) (Ajzen, 1985, 1991), it suggests that behavioral decisions are made based on reasoned considerations derived from available information. RAA states that behavioral intention (the readiness to engage in a behavior and hence determining the actual behavior) depends on attitude (positive or negative evaluations of behavior), perceived norms (perceived social pressure regarding the performance or non-performance of a certain behavior), and perceived behavioral control (PBC; perception of the ease or difficulty related to the performance of a certain behavior) (Fishbein \& Ajzen, 2010). To assess whether different behavior-change techniques (or a combination thereof) presented in an online gaming environment influence the explicit determinants of behavior - attitude, perceived norms, PBC, and intention towards the three behaviors of interest - these RAA variables were assessed in both before and after one, three, or five gaming sessions.

Explicit cognitions do not, however, exclusively predict behavior. Implicit associations, which are fast and activated automatically, are able to influence behavior outside conscious awareness (Gawronski, Hofmann, \& Wilbur, 2006; Greenwald \& Banaji, 1995; Hahn \& Gawronski, 2014), as opposed to explicit cognitions that are slow and deliberate (e.g., Strack \& Deutsch, 2004). For a variety of behaviors, such as eating (Friese \& Hofmann, 2009), aggression (Wiers, Beckers, Houben, \& Hofmann, 2009), and substance use (Curry, Scott, Bulotaite, \& Freng, 2017; Wiers \& Stacy, 2006), it has been shown that implicit cognitive factors account for unique variance (over and above explicit measures - see a meta-analysis by Rooke, Hine, \& Thorsteinsson, 2008). It has also been shown that 
negative implicit cognitions predict less consistent condom use (e.g., Grenard, Ames, \& Stacy, 2013; Stacy, Ames, Ullman, Zogg, \& Leigh, 2006) and condom use intentions in lowrisk scenarios (as compared to high-risk scenarios in which intentions were better predicted by explicit attitudes) (Czopp et al., 2004). The Implicit Association Test (IAT; Greenwald, McGhee, \& Schwartz, 1998) is widely used to measure implicit associations. Its predictive validity has recently been challenged in some studies (e.g., Blanton, Jaccard, \& Burrows, 2015; Jaccard \& Blanton, 2007; Rothermund \& Wentura, 2001) and confirmed in others (e.g., Horcajo, Rubio, Aguado, Hernández, \& Márquez, 201 Meyertheless, due to its robustness and reliability, it has become a widely used technique to measure the relative strength of implicit associations between two concepts (see a meta-analysis by Greenwald, PoehIman, UhImann, \& Banaji, 2009; Nosek et al., 2007).

\section{Working memory}

Working memory (WM) can be defined as the ability to maintain and manipulate goalrelevant information (Baddeley, 1992, 2012; Ma, Husain, \& Bays, 2014). It is part of a collection of cognitive functions - including planning, attention, and the inhibition of inappropriate actions - that are collectively known as executive control, and which allow people to take goal-directed actions (Baddeley \& Della Sala, 1996; Baddeley, 1998; Norman \& Shallice, 1986). A reduced WM capacity has been shown to hamper the proper regulation of automatic impulses, for instance, by shifting the regulation of drinking behavior from controlled processes to more reward-driven impulsive behavior (Friese \& Hofmann, 2009; Friese et al., 2008; Grenard et al., 2008; Hofmann, Gschwendner, et al., 2008), with most evidence in young people (Wiers et al., 2015). Accordingly, individuals with high levels of executive control functioning have shown attenuated associations between implicit cognition and sexual behavior (Ames et al., 2013; Grenard et al., 2013). As WM capacity influences the maintenance of goal-relevant information (Klingberg, Forssberg, \& Westerberg, 2002b; Ma et al., 2014), we were interested in whether training WM during one, three, or five sessions of an online game could influence the explicit behavioral determinants of our three behaviors of interest, making them more positive towards the respective behaviors.

\section{Selective response inhibition}

Efficient inhibitory control has been shown to influence the development of complex skills, such as arithmetic (Espy et al., 2004), reasoning (for a review, see Houdé \& Borst, 2015), theory of mind (Benson, Sabbagh, Carlson, \& Zelazo, 2013; Jacques \& Marcovitch, 2010), decision-making (for a review, see Cassotti, Aite, Osmont, Houde, \& Borst, 2014), creativity (for a review, see Cassotti, Agogué, Camarda, Houdé, \& Borst, 2016), and social 
adjustment (Diamond, 2013). Adapting mental processes to environmental demands, otherwise known as cognitive control, is another central aspect of goal-directed behavior (Logan \& Cowan, 1984; Miyake et al., 2000). Resolving competing demands often requires finding a balance between going or stopping, approaching or avoiding (Bari \& Robbins, 2013). The ability to control such impulses and to prevent acting on irrelevant information is known as inhibitory control (Van Veen \& Carter, 2006; Verbruggen \& Logan, 2008).

An important aspect of inhibitory control is the ability to overcome a prepotent response by stopping or even reversing an already initiated action (Logan, 1994; Logan \& Cowan, 1984). This inhibition of a prepotent response can be measured by the stop-signal task (SST). A similar paradigm, the go/no-go task (Newman, Widom, \& Nathan, 1985), also measures response inhibition. However, the main difference between both tasks is the timing of the presentation of a cue or event in the environment - known as a stopcue - relative to the go-stimulus (Reynolds, 2006). This stop-signal in the go/no-go task is presented unexpectedly in place of, or simultaneously to, the go-signal. This means that the task measures the inhibition of a planned response (action restraint) (Eagle et al., 2008). This inhibition occurs proactively by involving preparatory processes that influence whether an action will be initiated in the first place (Aron, 2011; Braver, 2012; Braver, Gray, \& Burgess, 2007; Chikazoe et al., 2009; Jaffard et al., 2008; Jaffard, Benraiss, Longcamp, Velay, \& Boulinguez, 2007; Verbruggen \& Logan, 2009; Vidal, Mills, Pang, \& Taylor, 2012). However, such inhibition can also be reactive - for example, the cessation of a motoric process in reaction to the stop-signal, - and something that occurs after the response process has been initiated. In the SST, the stop-signal is always presented after the go- cue so that this task measures the inhibition of an initiated action (action cancellation) (Schachar et al., 2007). Because response inhibition is a key component of executive control (Andrés, 2003; Miyake et al., 2000), we were interested in whether training task-relevant response inhibition (RI) - by pairing the stop-signal with the statement "unsafe sex" during one, three, or five sessions of an online game - could reduce impulsive approach decisions to appealing but health-compromising behavior, and therefore result in more positive evaluations of the three protective behaviors of interest, resulting in higher scores on the determinant measures of these behaviors.

\section{Evaluative conditioning}

Evaluative conditioning (EC) "refers to a change in the valence of a stimulus (the effect) that is due to the pairing of that stimulus with another positive or negative stimulus (the procedure)" (Hofmann, De Houwer, Perugini, Baeyens, \& Crombez, 2010a, p.390). In EC, a neutral stimulus is paired with a positive or negative stimulus, changing the valence of that stimulus (Bar-Anan, De Houwer, \& Nosek, 2012; De Houwer, 2007, 2011; De Houwer, Thomas, \& Baeyens, 2001; Hofmann, De Houwer, Perugini, Baeyens, \& Crombez, 2010; 
Jones, Olson, \& Fazio, 2010; Levey \& Martin, 1975; Martin \& Levey, 1978). As preferences have a strong impact on behavior and are learned rather than innate (Rozin \& Millman, 1987), learning to like or dislike something can have a pervasive impact on behavior, as preferences have been shown to influence attention, memory, and judgments (Fox, 2009; Gross \& Barrett, 2013). In the present study, we were interested in the question whether learning to like safer-sex decisions and to dislike unsafe decisions related to the three behaviors of interest could be achieved with EC. In our game, avatars controlled by participants decided whether or not to engage in safer-sex behavior. Directly after the player decided about the preference, the avatar who was to be engaged in the respective behavior received positive (or negative) audio and visual feedback. We were interested in whether this conditioning influenced the evaluation of further similar stimuli about engaging or not in the three protective behaviors.

In the present study, we tested whether EC, task-relevant RI training, and WM training presented in a serious game would result in more positive explicit and implicit evaluation of the three protective behaviors. More precisely, before playing the serious game, both participant's explicit behavioral determinants and implicit associations towards the three protective behaviors were assessed. Participants were then randomly assigned to different conditions (consisting of various combinations of active and mock versions of EC, taskrelevant $\mathrm{RI}$ training and WM training, and of different numbers of playing sessions). After the intervention, both participants' explicit behavioral determinants and implicit associations toward the three protective behaviors were reassessed. First, we predicted that mock only conditions would not result in any significant differences between pre- and post-intervention measures. Second, we predicted that a combination of active versions of EC, task-relevant RI training, and WM training would lead to stronger explicit determinants of - and more positive implicit attitudes towards - the three protective behaviors as compared to any other combination of active and mock versions. Third, we predicted that participants who played five sessions would show more favorable explicit evaluations of - and implicit attitudes towards - the three protective behaviors as compared to participants who played only three sessions. Furthermore, participants who played three sessions would show more positive explicit and implicit determinants of the three behaviors than participants who only played the game once.

\section{Methods}

\section{Participants}

Participants were recruited from different vocational and high schools in Germany as well as from Maastricht University. Following approval from each institutional ethics committee, researchers informed participants about the study in general terms during a short visit to their classroom. Furthermore, posters and flyers in common areas such as the 
refectory and entry hall also displayed a quick response code (a so-called QR-code consisting of a matrix barcode that can be scanned by smartphones and links to a website) that allowed participants to get more information about the study, in particular about the inclusion criteria of being heterosexual, aged between 18 and 24 years, sexually active, and not currently in a relationship. After giving their written informed consent, participants were linked to a secured website that contained the study materials. They were randomly assigned to play only one session, to play a total of three sessions on three consecutive days, or to play a total of five sessions on five consecutive days. Participants could quit the study at any time (without providing a reason and without any consequence). Anonymity was assured as the e-mail addresses participants used to sign-up were not linked to individual data. In total, 224 individuals signed up, but 29 did not finish the study, meaning a total of 195 individuals completed the study. These participants were aged between 18 and 24 years $(M=20.7 S D=1.29) ; 135$ (6 9 \% \%) were female, 60 (31\%) were male. All were sexually active, heterosexual, and not in a relationship (where STI reducing safer-sex behavior plays a diminished role as compared to increased contraceptive measures, see, e.g., Bauman \& Berman, 2005; Manlove, Ryan, \& Franzetta, 2004; Manlove \& Terry-Humen, 2007) at the time of the study. In exchange for their participation, participants who played one time received $€ 25$, participants who played three times received $€ 50$, and participants who played five times received $€ 75$ after they had completed the study. The money was delivered in person during scheduled meetings at the respective school or university.

\section{Procedure}

This two-part study was conducted entirely online, and procedures were explained in detail to the participant on a web page prior to the study. Participants were randomly assigned to one of 13 conditions:

- 1-3: EC active, RI training active, and WM training active with one, three, or five gaming sessions,

- 4-6: EC active, RI training active, and mock WM training with one, three, or five gaming sessions,

- 7-9: EC active, mock RI training, and mock WM training, with one, three, or five gaming sessions,

○ 10-12: Mock EC, active RI training, and mock WM training, with one, three, or five gaming sessions,

- 13: Mock EC, mock RI training, and mock WM training with five gaming sessions.

The first part of the study started with a short demographic questionnaire, assessing age and biological sex. Participants then completed a unipolar Single Category Implicit Association Test (SC-IAT, see Karpinski \& Steinman, 2006) concerning the three behaviors of interest. The order of the SC-IATS was counterbalanced. Next, two blocks of questionnaires measured - in counterbalanced order - RAA variables (intention, attitude, social 
norm, and perceived behavioral control) related to the three behaviors of interest. Participants were then linked to the online game (designed and programmed in Hamburg, Germany, by the University of Applied Sciences Hamburg) where they were asked to create an account and choose their login and password. Participants in the conditions with multiple gaming sessions were asked to log in to their account to start the next session, where they could continue at the same level they had reached when they stopped playing at the end of a previous session. Having created an account, participants were asked to build their own avatar in order to increase their engagement in the game (Birk, Atkins, Bowey, \& Mandryk, 2016; Christou \& Michael, 2014; Lindgren, 2013). We decided that this would be beneficial even though it has been shown that a participant's input on game aesthetics has only a limited influence on its effectiveness (DeSmet et al., 2016). They could opt for a female or a male avatar and were free to choose among five different style options for caps, hairstyles, shirts, trousers (and skirts for females), and shoes. After participants had created their avatar, the next screen was presented. They could see their avatar sitting between two friends on a terrace (Image1). A dialogue starts by displaying speech bubbles in which both friends suggest that they go out that night and try to have sex with men (for female avatars) or women (for male avatars). After agreeing to that plan, the next screen shows the avatar standing in front of a bar in a dancehall, together with several other individuals of the opposite sex (Image 2). The upper right corner of the screen shows the amount of points a participant collected. In the game, health-sustaining behavior earns points for the participant whereas behavior that jeopardizes health leads to a reduction of points. Other screens where the avatar could meet computer-generated individuals of the opposite sex are the restrooms (Image 3) and the dancefloor (Image 4).

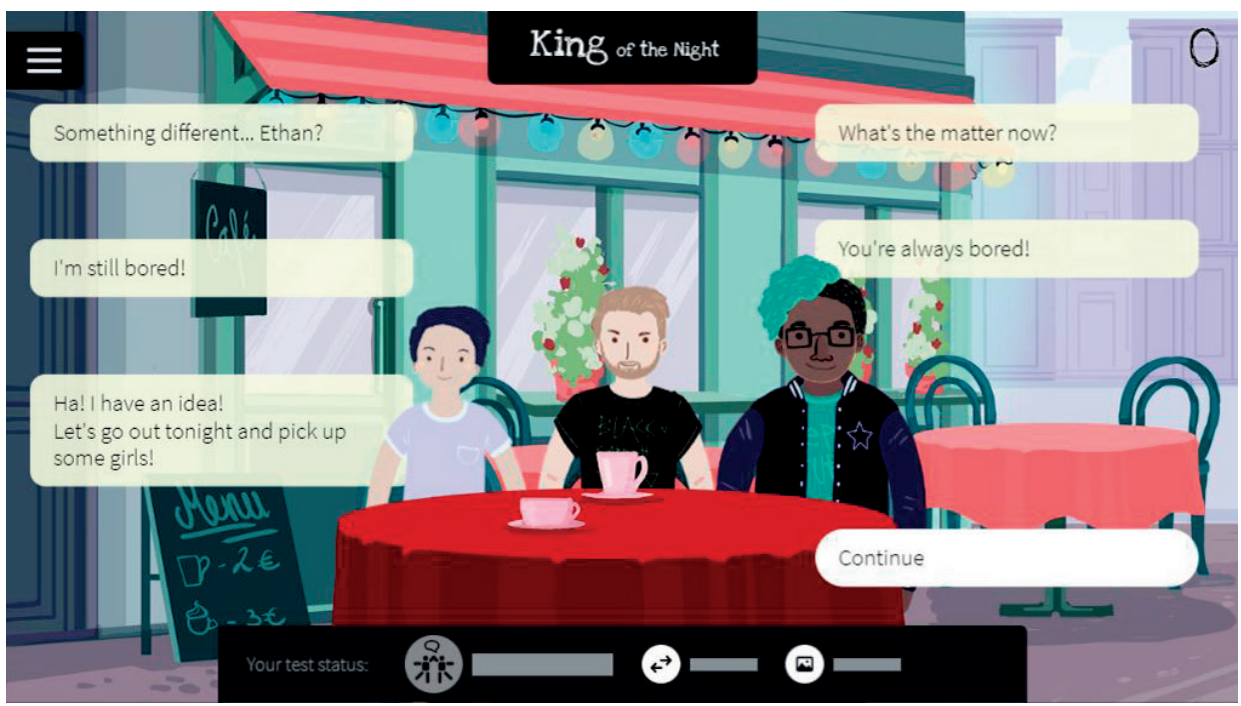

Image 1. The avatar sits together with friends on a terrace while they plan trying to have sex when they go out that evening. 


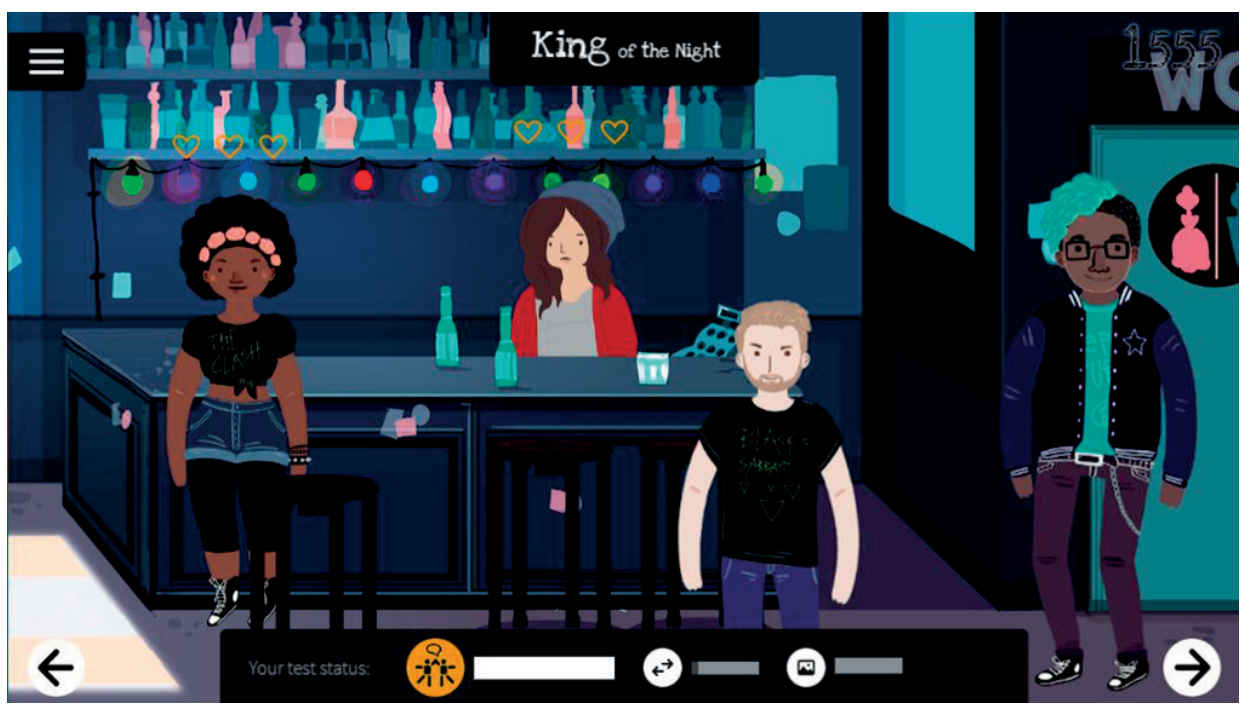

Image 2. The bar

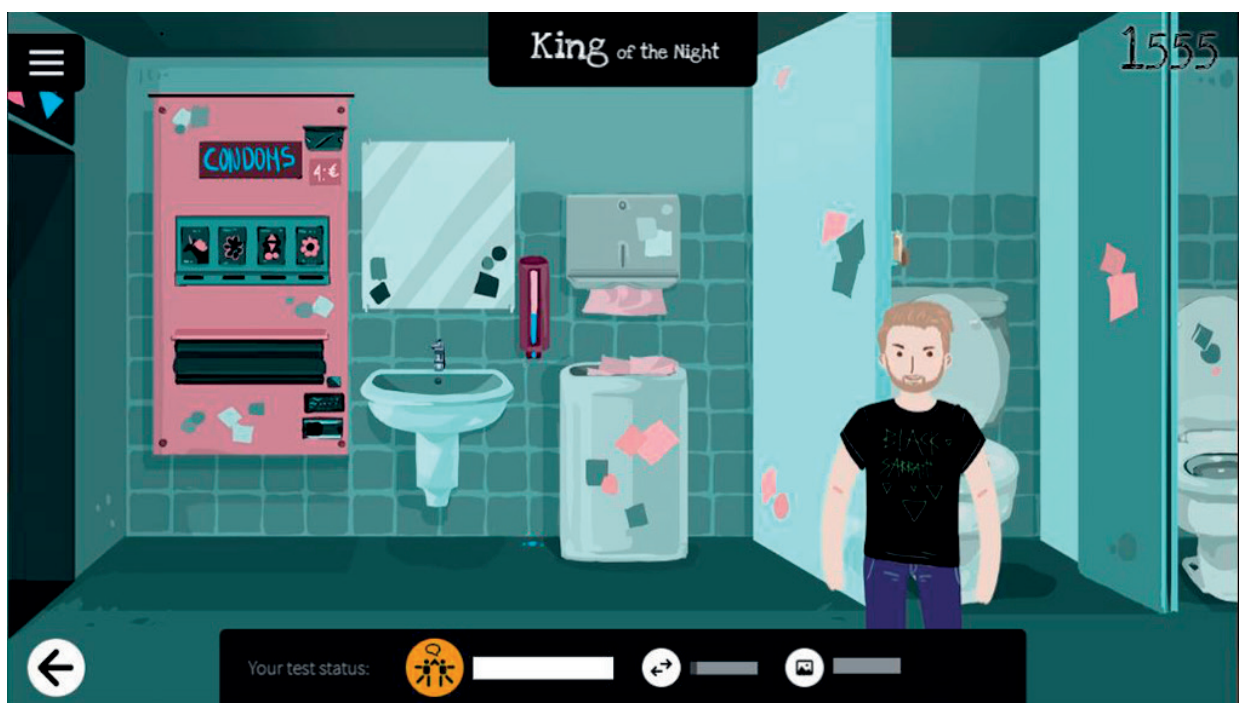

Image 3. The restrooms 


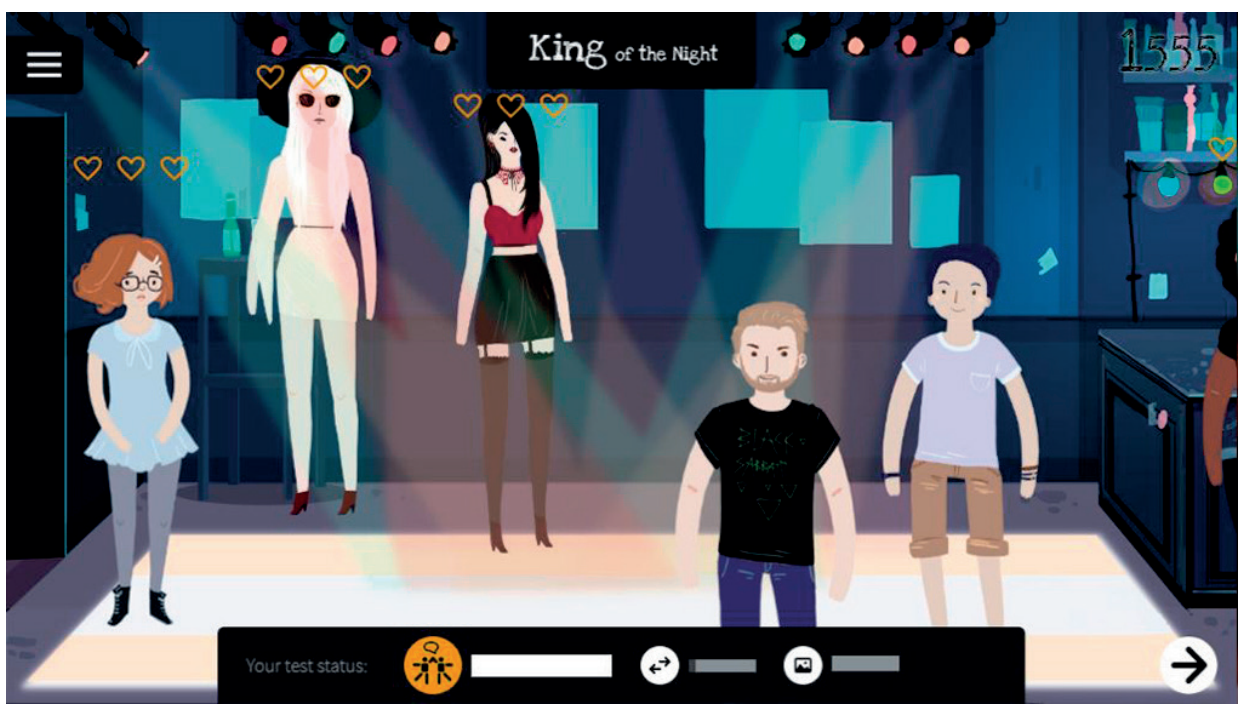

Image 4. The dancefloor

\section{Evaluative conditioning}

Clicking on an individual resulted in the individual posing a question (related to one of the three behaviors of interest) to the participant's avatar (e.g., "Do you feel like playing villain and hero? In your bedroom? The hardcore version?"). The participant could then choose between two answers by clicking on the speech bubble (e.g., "No thanks, I don't like hardcore." or "Yeah, sure, that's my favorite game!", see Image 5). The response triggered an instant pairing of the answer with a visual stimulus (green if the answer was health-sustaining or red if the answer could endanger the avatar's health), an auditory stimulus (a pleasant bell sound for a health-sustaining answer or an unpleasant horn sound for a health-endangering answer), and a reward element (adding points for a health-sustaining answer together with the message "Good answer! You win 100 points!" or reducing points for a health-endangering answer together with the message "Oops! You lose 100 points.", see Images 6 and 7, respectively). This evaluative conditioning was presented randomly 25 times per health behavior of interest (always displaying different questions). A total of $75 \mathrm{EC}$-instances were therefore presented across the three behaviors during one gaming session. Depending on previous dialogue with a computer-generated individual, the participant's avatar could end up in the individual's bedroom (Image 8) or in the backseat of a car (Image 9). In these two scenarios, depending on the participants choice of answer for the avatar, the visual stimulus, auditory stimulus, and reward element remained the same, but the written feedback changed to "You have safe sex and win 400 points!" for health sustaining behavior and "You have unsafe sex and lose 100 points" for health-endangering behavior (see Images 10 and 11, respectively). Before the screen changed to the bar setting again, a short info screen appeared that stated "You had sex!", independent of the previous behavior. Thus, this screen was a reminder that 
the avatar just had engaged in sexual intercourse, leaving to the player's discretion how safe or unsafe this intercourse might have been (Image 12).

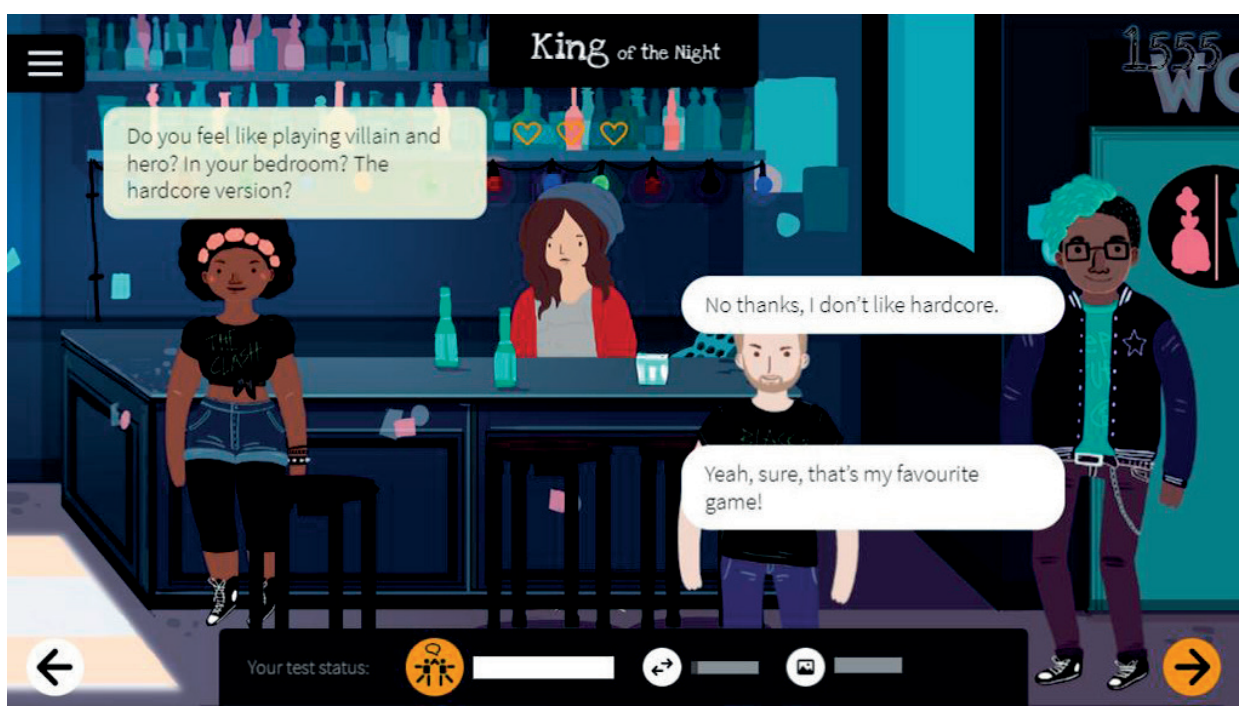

Image 5. An example of answer possibilities.

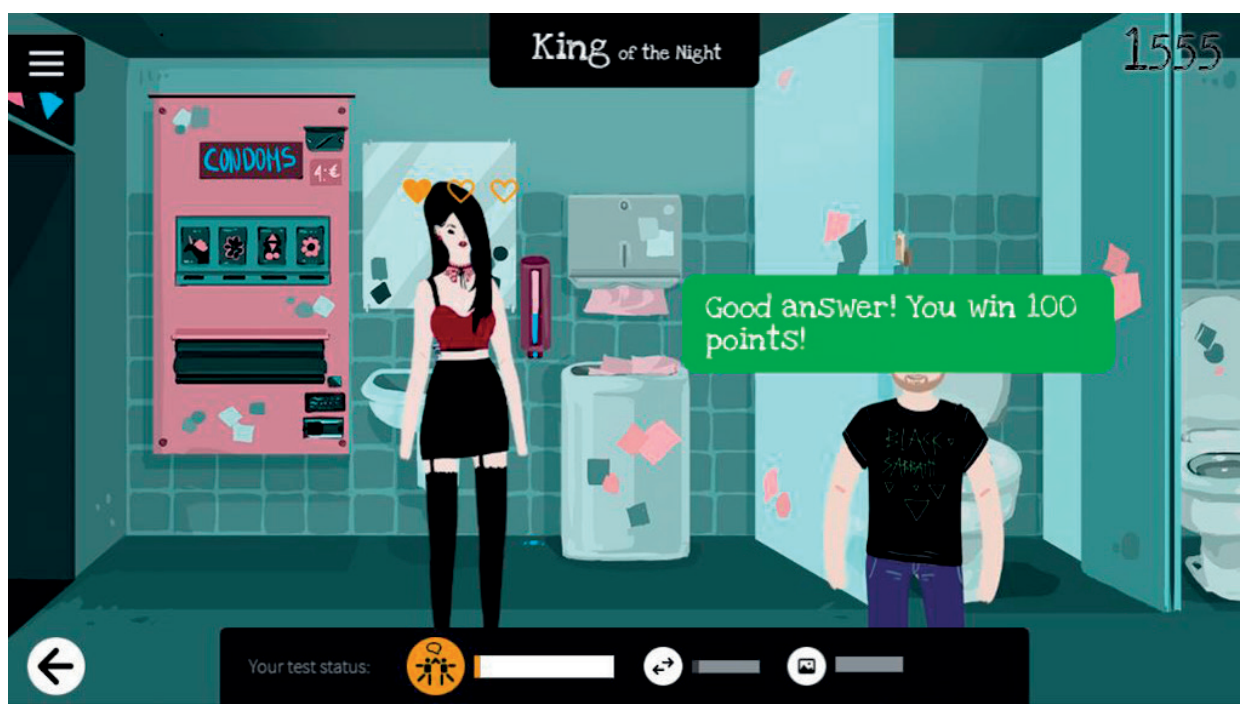

Image 6. Evaluative conditioning to like a health-sustaining decision. 


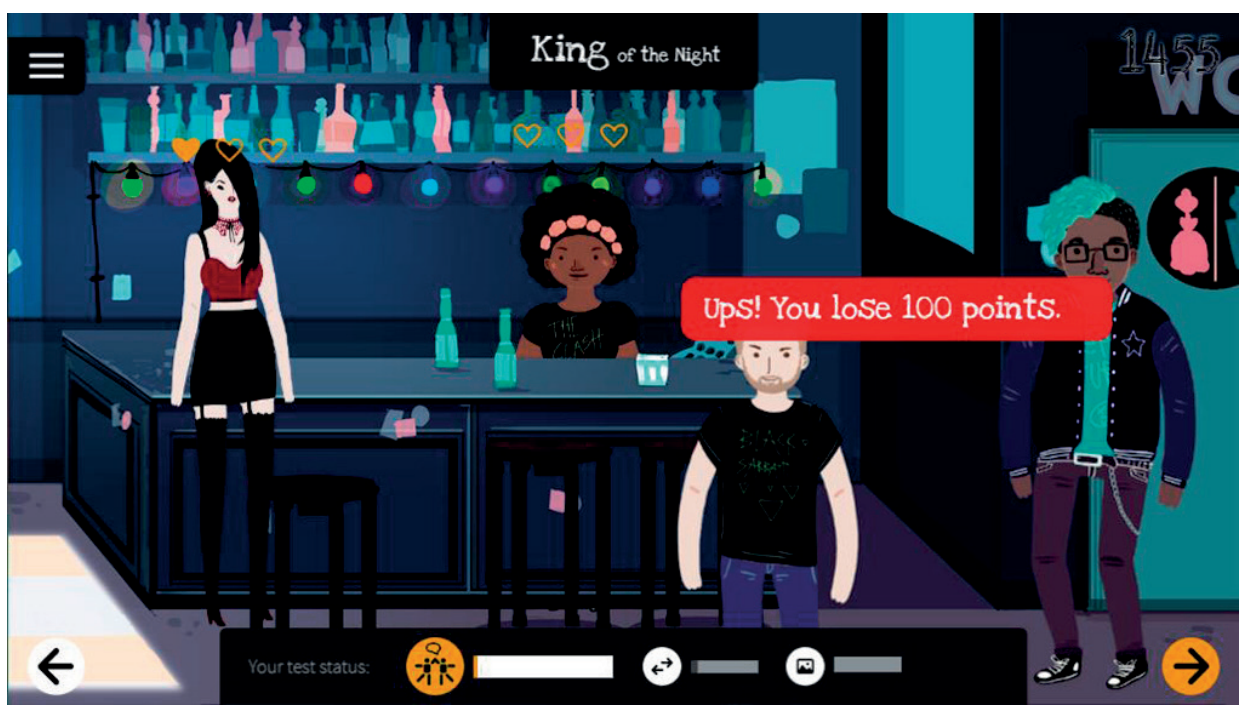

Image 7. Evaluative conditioning to dislike a health-endangering decision.

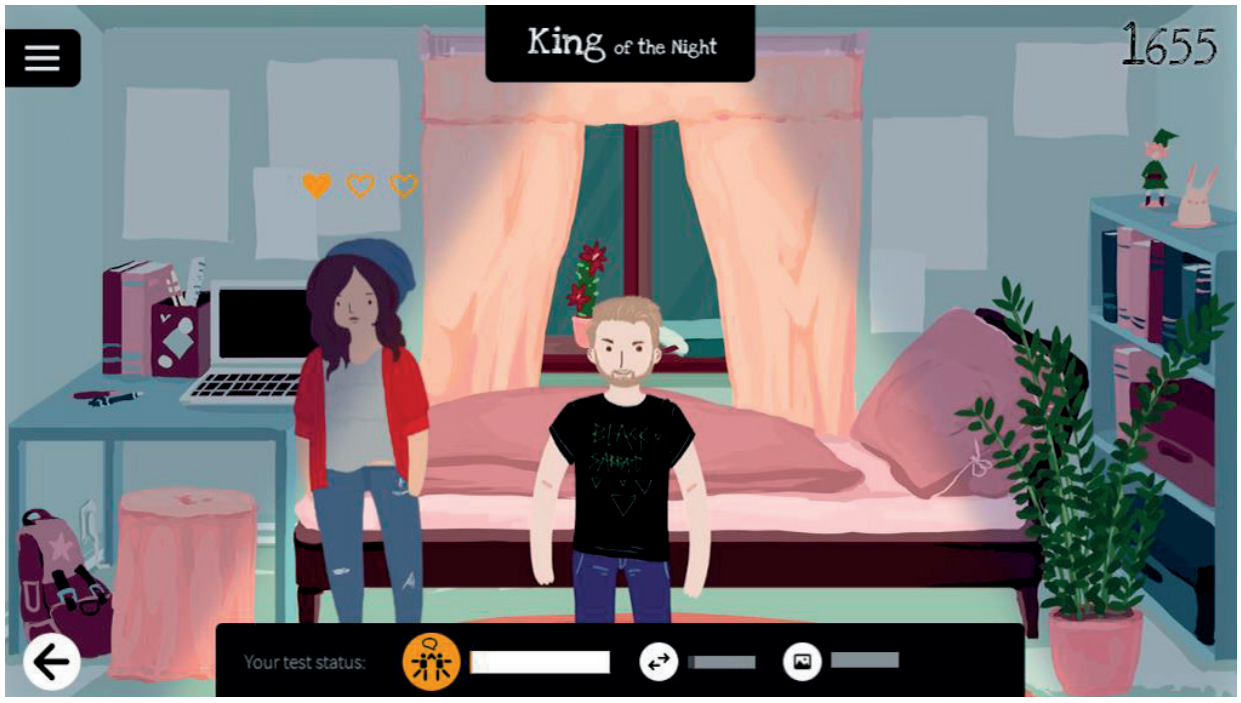

Image 8. At home having the possibility to engage in sexual intercourse. 


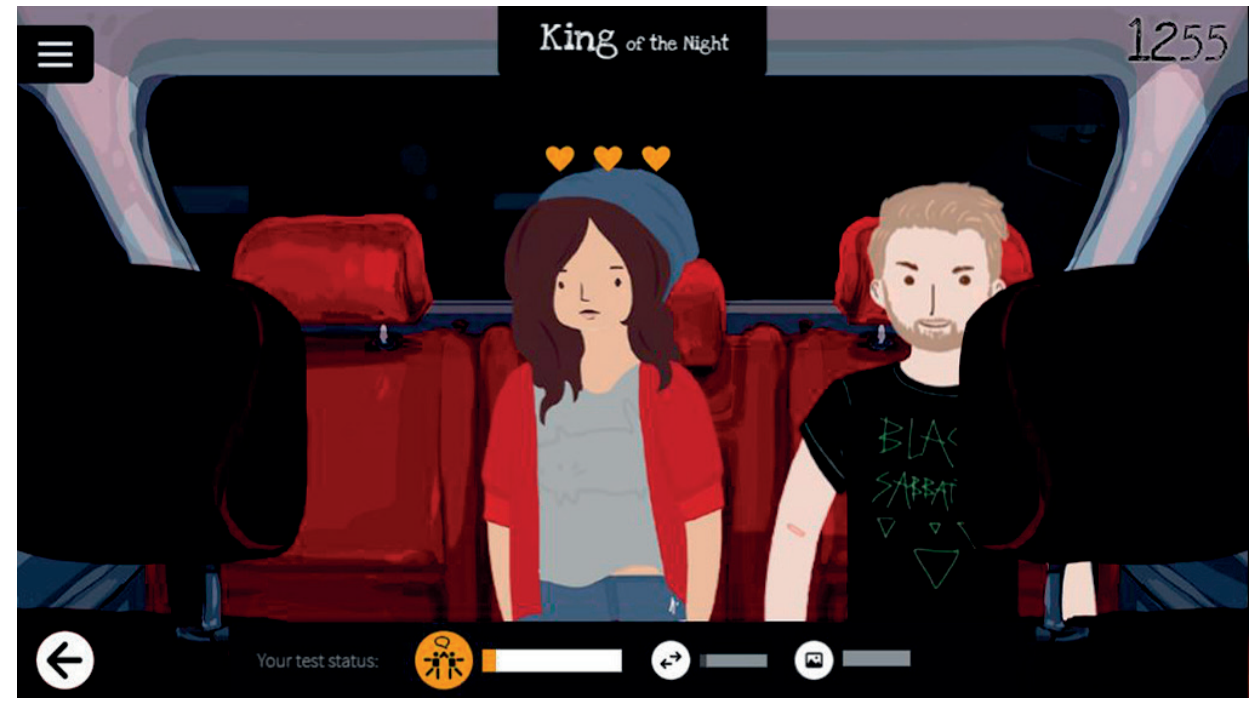

Image 9. In a car having the possibility to engage in sexual intercourse.

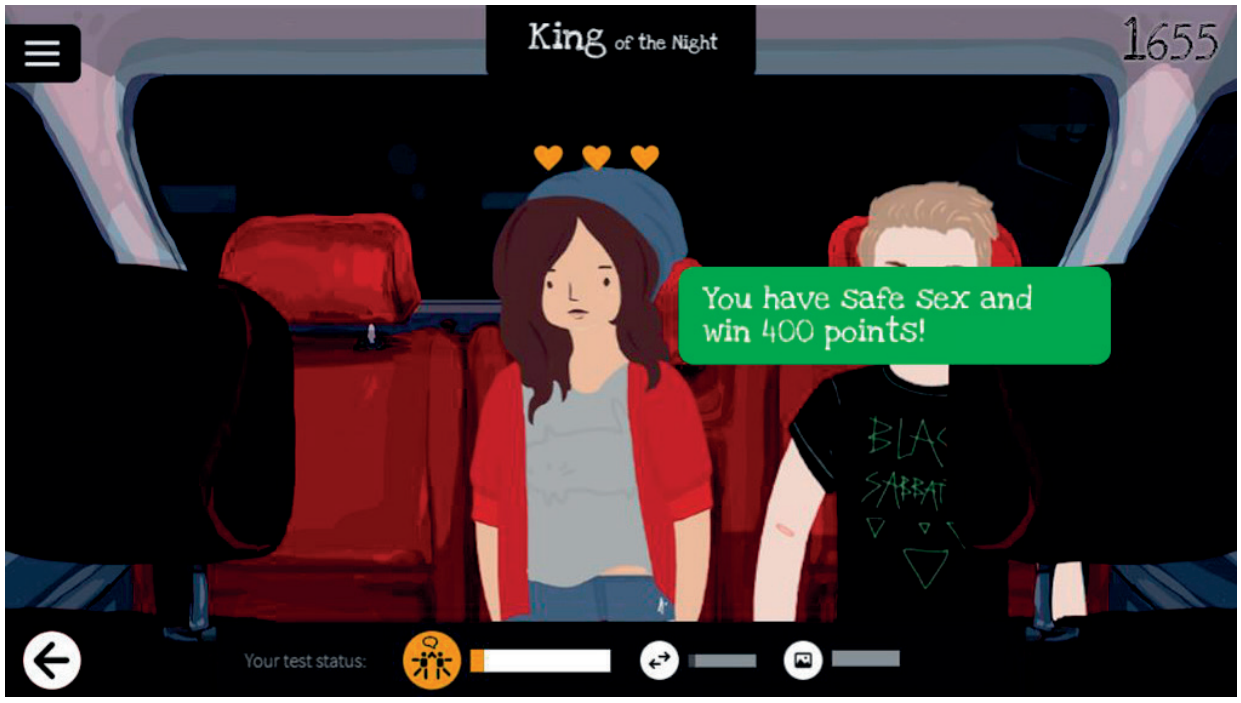

Image 10. Evaluative conditioning to like having safe-sex. 


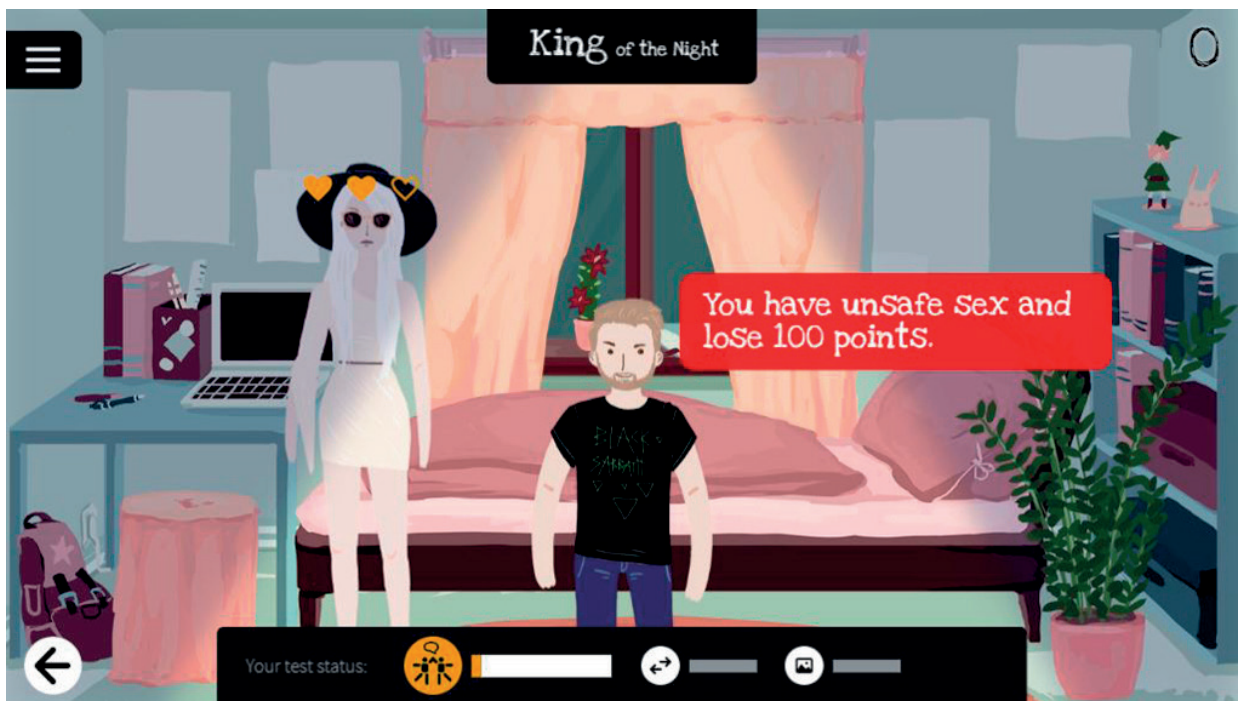

Image 11. Evaluative conditioning to dislike unsafe-sex.

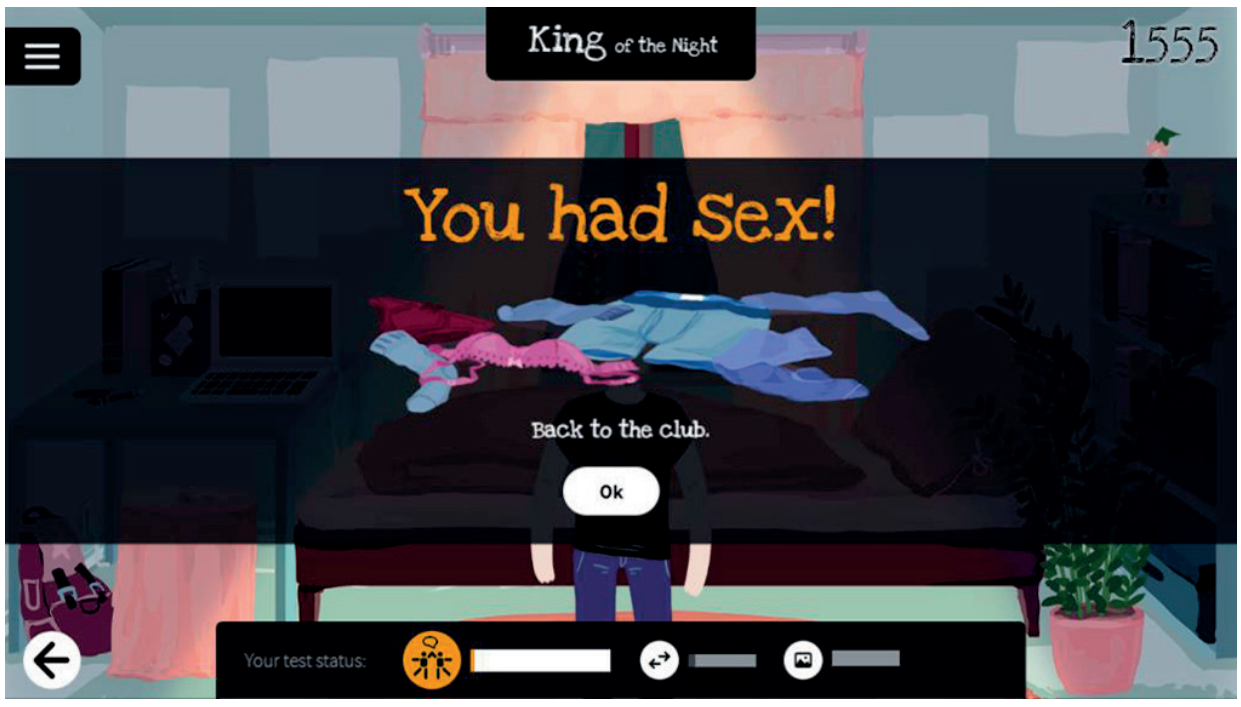

Image 12. Feedback after the avatar had sexual intercourse.

\section{Selective response inhibition training}

The selective response inhibition training consisted of five blocks that were randomly presented throughout each gaming session. The go task was to respond to a green arrow that was paired with the statement "safe sex" (presented above the arrow to increase task relevance) in green font (Image 13). If the green arrow pointed to the left, participants had to press the " $x$ " key on the keyboard or on the " $x$ " field at the left side of a touchscreen device, and if the green arrow pointed to the right, they had to press the " $\mathrm{m}$ " key on a 
keyboard or on the " $\mathrm{m}$ " field at the right side of a touchscreen device. The stop-signal was a red arrow that pointed or to the left or to the right, paired with the statement "unsafe sex" (also presented above the arrow) in red font (Image 14). The stop-signal indicated to participants that they should withhold their response for that particular trial. Each trial began with the presentation of a fixation cross, displayed for 500 ms, followed by the random presentation of a green or red arrow-statement pair. Subjects were instructed to respond as quickly and accurately as possible by pressing the respective key or field. The arrowstatement stimulus remained on the screen for $850 \mathrm{~ms}$, followed by a 1,000 ms inter-trial interval during which the screen was left blank. The occurrence of stop-signals was counterbalanced and accounted for $20 \%$ or $40 \%$ of the trials in a block. Signals were presented either as a full stop-signal or as a stop-signal delay (SSD), which is the delay between the presentation of the go stimulus and the stop-signal used to manipulate the probability of inhibition (Logan \& Cowan, 1984). During the latter trial, the originally presented go-task (green arrow-statement combination) was changed into a stop-signal (red arrow-statement combination) with an SSD of $250 \mathrm{~ms}$. The SSD was further continually adjusted following a tracking algorithm, increasing by 50 ms after each signal-inhibit trial and decreasing by 50 ms after each signal-response trial in order to reduce predictability. Participants were given 20 practice trials during which they had to respond with an accuracy above $75 \%$ and reaction times below 1,000 ms. Practice trials were continued until these thresholds were met. Following the practice sessions, participants completed the main task that consisted of five blocks, randomly presented throughout the gaming session. Each block consisted of 240 trials. In the mock condition, participants only had to press or the " $x$ " or the " $m$ " key (or the respective fields on a touchscreen device) if an arrow-statement combination appeared on screen, without paying attention to the color or the pointing direction of the arrows.

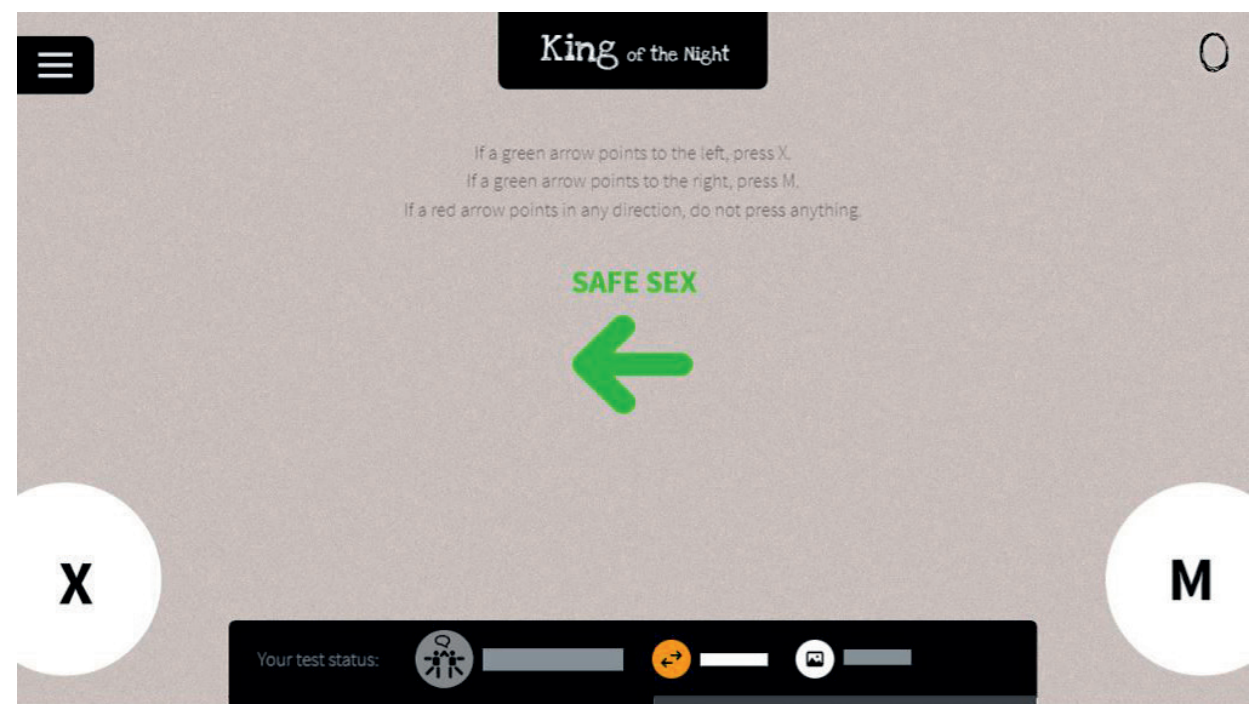

Image 13. Go-signal in the Stop-Signal Task with a task-relevant message. 


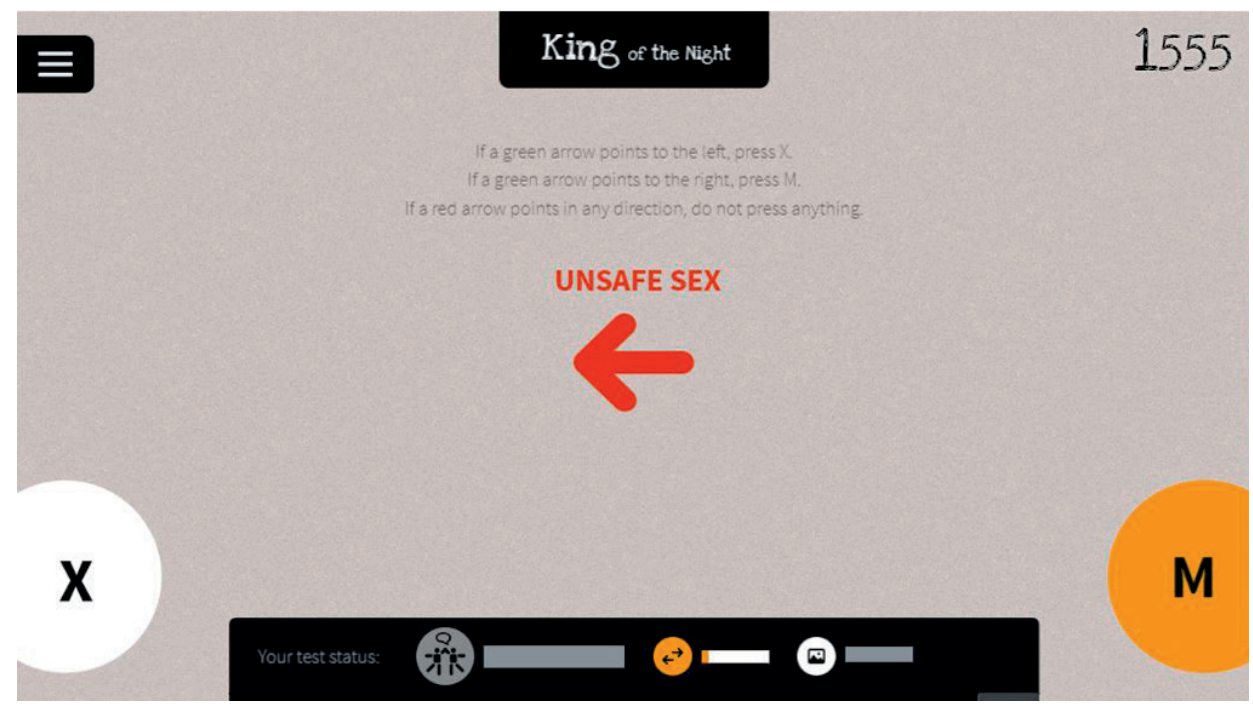

Image 14. Stop-signal in the Stop-Signal Task with a task-relevant message.

\section{Working memory training}

Participants completed a letter span task consisting of five blocks (adapted from Klingberg, Forssberg, \& Westerberg, 2002) in which a random letter was presented and paired with an object (e.g., a bottle) on the computer screen for 1 second. First, three object-letter pairs were presented. After the object-letter pairs had disappeared, one previously presented object was presented, and participants were required to identify the letter that was initially paired with the object using the keyboard (Image 15). The block difficulty was automatically adjusted on a trial-by-trial basis. The number of the object-letter pairings increased and decreased according to participants' performance, starting with a sequence of three pairs. When participants correctly reproduced the object-letter pairing on two consecutive trials, one object-letter pair was added to the sequence of the next trial. However, when participants did not correctly reproduce the sequence on two consecutive trials, the sequence in the next trial contained one item fewer. The block was finished after participants correctly identified the corresponding letter of one randomly selected object-letter pairing out of nine possible letters. The training lasted for a total of five blocks that were randomly presented throughout one serious game session. In the mock condition, objects were presented without a letter pairing, and instead of identifying a correct letter, participants only had to identify the number of objects that had previously been presented.

\section{Dance battle}

To avoid monotonous repetition and allow participants to have a break, a "dance battle" was randomly presented five times during one gaming session. Here, the participant had 
to move the avatar in such a way that he or she copied the dance movements of another (computer-generated) avatar by using the specific keys on a keyboard or by pressing the relevant fields on a touchscreen device. To engage participants more in the game, good performance in the dance battle was rewarded with additional points (Image 16).

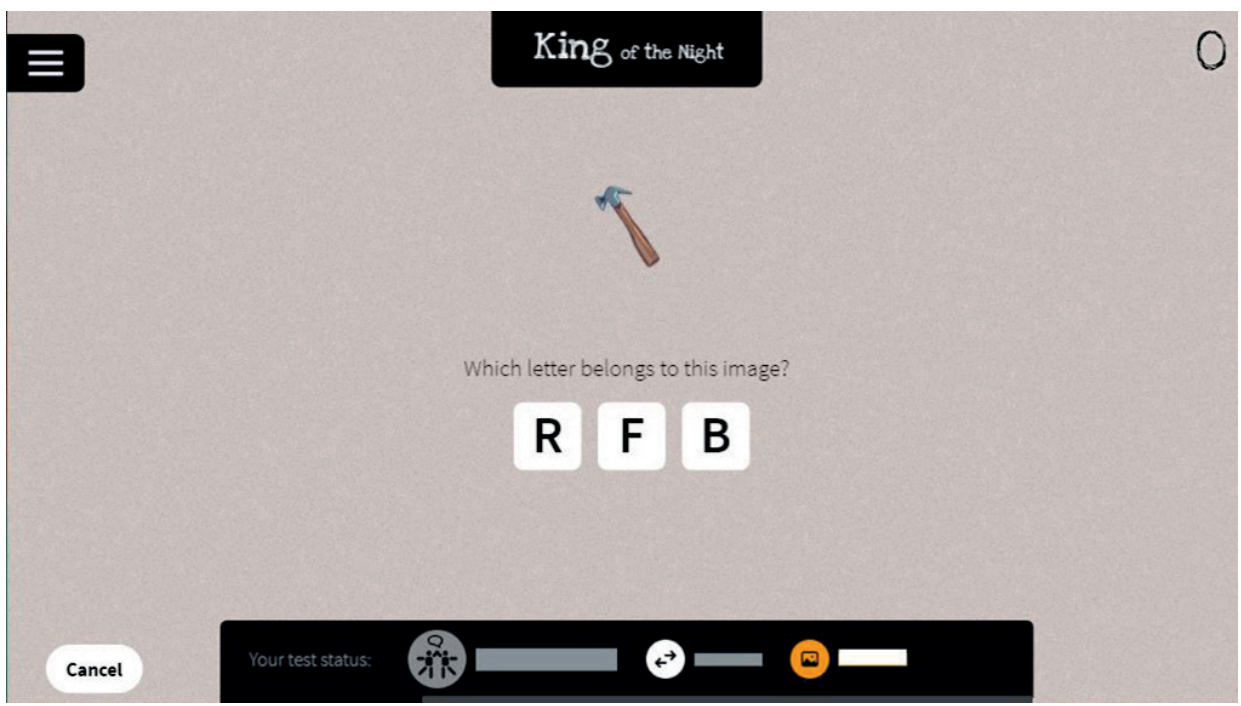

Image 15. Starting screen of the Working Memory training task.

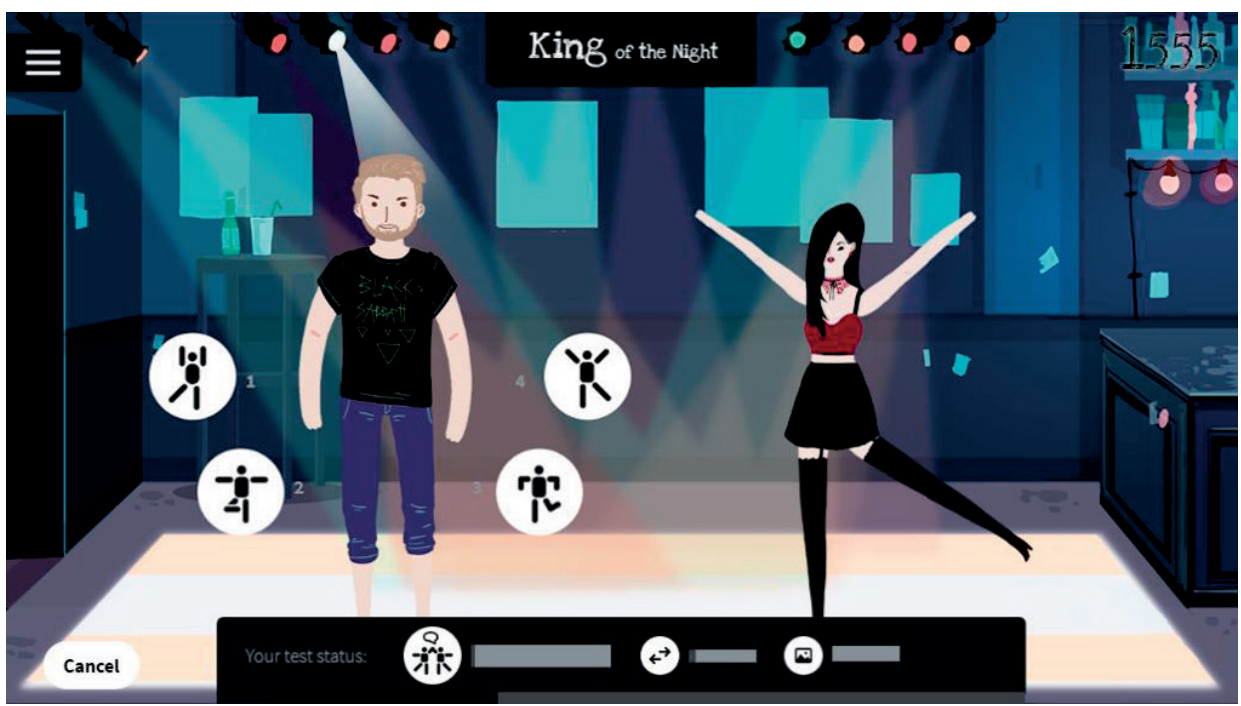

Image 16. The dance battle. 


\section{Final assessment}

After finishing the first part participants were thanked and those who took part in a condition that consisted of three or five gaming sessions were reminded that the next day they would receive an e-mail containing a link to their gaming account from which they could continue the game with the same amount of points they had earned during the previous session. After participants had finished all gaming sessions, they were reminded that the final part of the study would take place two weeks later. This final part consisted of the same explicit and implicit measures as those taken in the first part. After these SC-IAT measurements had been taken and the RAA questionnaire had been filled in again, participants were thanked and received their voucher codes online. The total duration of the first session (including all assessments, setting-up an account, preparing for the game and playing it) was approximately 60 minutes. Each gaming session lasted about 45-60 minutes, and the final assessment lasted for approximately 30 minutes. This means a total participation time of around 190 minutes for participants who played the game only once, 270 minutes for participants who played the game three times, and 390 minutes for participants who played five sessions.

\section{Measures}

Implicit associations

Implicit associations can be measured indirectly during a computerized categorization task. This is done without asking participants to reflect and report their motivations for a specific behavior. IATs are based on the principle that people find it easier to categorize stimuli together if those stimuli are strongly associated as compared to stimuli that are only weakly (or not) associated. However, most IATs are bipolar and therefore always require two concepts. To overcome this restriction, the so-called unipolar Single Category IAT (SC-IAT) has been introduced by Karpinski and Steinman (2006) in which only one attribute category is presented at a time. Using a SC-IAT, therefore, allowed us to use three blocks (instead of five in a regular IAT) in which stimuli representing only one target category share a key with an evaluative concept (e.g., positive words). The other key is then used to respond to the opposite evaluative concept stimuli (e.g., negative words). A single-category implicit evaluation score can then be computed by comparing response latencies between blocks where the target category is paired with positive stimuli and blocks where the target category is paired with negative stimuli. In the present study, we decided to use SC-IATS to assess participants' implicit attitudes toward the three protective behaviors of interest because IATs would have created an artificial bipolarity in the assessment of the targeted behaviors (assuming, for example, that there is an opposite concept to condom use).

One of our SC-IATs measured implicit attitudes toward condom use with a new sex partner, one measured implicit attitudes towards asking a new sex partner about his/her sexual health status, and one measured implicit attitudes towards using less risky sex practices 
during first-time sex with a new sex partner. After one SC-IAT had been completed, participants could take a break. Stimulus words for using condoms, asking a new sex partner about his/her sexual health status, and refraining from risky sex practices with a new sex partner were selected based on a pilot study in which 20 noun-verb pairs (e.g., use condoms) per target behavior were presented to 20 undergraduate students. For each noun-verb pair, they were asked to rate on a 7-point Likert-scale how much this noun-verb pair described the target behavior (ranging from [1] "not at all" to [7] "very much"). Finally, seven nounverb pairs - per target behavior - with a minimum score of five were used as stimulus material (see Table 1). Positive attribute words were: happiness, harmony, joy, and safety whereas negative attribute words were: grief, hatred, harm, and stress.

Table 1. Word pairs per target behavior out of which participants were asked to choose four per respective SCIAT. The English translation of these word pairs is not always accurate as some colloquial expressions cannot be literally translated from German without losing the original meaning.

\begin{tabular}{|c|c|c|}
\hline & Original German text & English translation \\
\hline \multirow[t]{7}{*}{ Condom SC-IAT } & Kondom gebrauchen & to use a condom \\
\hline & Präservativ gebrauchen & to use a preservative \\
\hline & Pariser gebrauchen & to use a Johnny \\
\hline & Verhüterli gebrauchen & to use a sheath \\
\hline & Gummi gebrauchen & to use a rubber \\
\hline & Lümmeltüte gebrauchen & to use a raincoat \\
\hline & Präser gebrauchen & to use a prophylactic \\
\hline \multirow[t]{7}{*}{ Asking SC-IAT } & Gesundheit abfragen & to inquire about health \\
\hline & Geschlechtskrankheiten abfragen & to inquire about sexually transmitted diseases \\
\hline & Gesundheitsrisiken nachfragen & to ask about health risks \\
\hline & STIs nachfragen & to ask about STIs \\
\hline & Gesundheitsstatus abfragen & to inquire about health status \\
\hline & Gesundheitszustand nachfragen & to ask about health status \\
\hline & Gesundheitsstörungen nachfragen & to ask about health problems \\
\hline \multirow[t]{7}{*}{ Practices SC-IAT } & Sexpraktiken anpassen & to adapt sex practices \\
\hline & Stellungen anpassen & to adapt sex positions \\
\hline & Stellungen aussuchen & to choose sex positions \\
\hline & Sexpraktik aussuchen & to choose sex practices \\
\hline & Stellung angeben & to specify sex position \\
\hline & Sextechnik wechseln & to change sex technique \\
\hline & Stellung wechseln & To change sex position \\
\hline
\end{tabular}

Before the respective SC-IAT, and to account for possible individual differences in concept representation, each participant was asked to select four out of the seven noun-verb pairs that best represented best the respective target behavior. These 4 noun-verb pairs were then used in the SC-IAT for that particular participant. The affective label words were always presented at the top of the screen, appropriately positioned on the left or the right side of the screen, depending on the required response (see Greenwald, 
McGhee, \& Schwartz, 1998). Each SC-IAT consisted of three blocks that came in a fixed order (see Table 2). During block 1, the attribute discrimination phase, participants were instructed to press the left key if the stimulus word that comes up in the middle of their computer screen is a positive word (e.g., happiness) and to press the right key if the stimulus word is negative (e.g., grief).

In block 2, the combination phase, participants were instructed to press the left key if the stimulus word is a positive word and press the right key if the stimulus word is a negative word or a safer-sex behavior related noun-verb pair (e.g., use condom). In block 3 , the reversed combination phase, participants press the left key if the stimulus word is a positive word or a safer-sex behavior related word and press the right key if the stimulus word is a negative word. The assignment left vs. right of positive, negative, and safer-sex behavior related noun-verb pairs was fully counterbalanced. The IAT tasks were programmed in Inquisit 2.0 (Millisecond Software, USA). We chose D600 scores as main outcome measures for the IAT (Greenwald, Nosek, \& Banaji, 2003). This algorithm includes all trials (including the attribute discrimination phase) and gives an error penalty of $600 \mathrm{~ms}$ to the reaction times on erroneous responses. The outcome is then divided by a personalized standard deviation of the combination phases so that the measure is not influenced by differences in response speed between participants. Higher D600 scores reflect a more positive association between implicit attitudes and safer-sex behavior related noun-verb pairs.

Table 2. Schematic overview of the block sequence in the Single Category-Implicit Association Tests (SC-IATs) measuring implicit attitudes toward condom use with a new sex partner (Condom SC-IAT), measuring implicit attitudes towards asking a new sex partner about his/her sexual health status (Asking SC-IAT), and measuring implicit attitudes towards using less risky sex practices during first-time sex with a new sex partner (Practices SC-IAT).

\begin{tabular}{|c|c|c|c|c|c|c|}
\hline \multirow[b]{2}{*}{ Condom SC-IAT } & \multicolumn{2}{|c|}{$\begin{array}{l}\text { Block } 1 \\
\text { Attribute discrimination }\end{array}$} & \multicolumn{2}{|c|}{$\begin{array}{l}\text { Block } 2 \\
\text { Combination }\end{array}$} & \multicolumn{2}{|c|}{$\begin{array}{l}\text { Block } 3 \\
\text { Reversed combination }\end{array}$} \\
\hline & positive & negative & positive & $\begin{array}{l}\text { negative } \\
\text { condom word }\end{array}$ & $\begin{array}{l}\text { positive } \\
\text { condom word }\end{array}$ & negative \\
\hline Asking SC-IAT & positive & negative & positive & $\begin{array}{l}\text { negative } \\
\text { health word }\end{array}$ & $\begin{array}{l}\text { positive } \\
\text { health word }\end{array}$ & negative \\
\hline Practices SC-IAT & positive & negative & positive & $\begin{array}{l}\text { negative } \\
\text { practices word }\end{array}$ & $\begin{array}{l}\text { positive } \\
\text { practices word }\end{array}$ & negative \\
\hline
\end{tabular}

\section{Explicit cognitions}

RAA scores on items that were included to assess the same variable were averaged into one measure in cases where internal consistency was sufficient (Cronbach's alpha > .60). Participants' intentions, attitudes, subjective norms, and perceived behavioral control towards the three safer-sex behaviors (i.e., to use condoms, ask about the current sexual health status, and refrain from risky sex practices with a new sex partner) were each assessed by two items using 7-point Likert scales. The appendix provides a list of all RAA items, and the response scales used. 


\section{Data analysis}

Data were analyzed for completers only, using the predictive analytic computer software SPSS, version 25.0 (Statistical Product and Service Solutions, IBM, New York). As our study did not use a full factorial design, we could not test for a possible interaction between all conditions and all groups. Therefore, after a descriptive analysis, we first used a multivariate analysis of variance (MANOVA) to determine whether there was a significant difference between the number of gaming sessions on all questionnaire data of all three behaviors per condition. Then, a repeated measures Analysis of Variance (ANOVA) was used to determine the effect of condition and number of gaming sessions on attitudes, social norms, perceived behavioral control, and intention to engage in each of the three protective behaviors. SCIAT D600 scores were then analyzed with a MANOVA to determine whether there was a significant difference between the number of gaming sessions on the combined D600 scores of all three behaviors per condition. In case of a significant multivariate effect, univariate analyses were conducted for each behavior and session. Lastly, we tested for effects of active evaluative conditioning, active selective inhibition training, and active working memory training on explicit attitudes, social norms, perceived behavioral control, and intention to engage in each of the three protective behaviors compared to their mock conditions (main effects), irrespective of whether other conditions were also active or not. Therefore, we used planned contrasts with a Bonferroni adjustment (alpha corrected to .025) that were conducted separately for one, three, and five gaming sessions.

\section{Results}

Active evaluative conditioning, active behavior inhibition, active working memory training

\section{Questionnaire data}

With all three components active (evaluative conditioning, selective inhibition training, and working memory training), a MANOVA showed a statistically significant difference between the number of sessions completed on combined attitudes, $F\left(\begin{array}{lll}6,8 & 0\end{array}\right) .948, p<$ .05 ; Wilks' $\Lambda=.662$; partialln8 6 ; on combined perceived nornf( $(5), 80)=3.048$, .05 ; Wilks' $\Lambda=.673$; partiall1n 80 ; and on combined intentiof( $\$ 9,80)=4.787$, .001 ; Wilks' $\Lambda=.580$; páatial ३ 9 ; but not on combineetceived behavioral control

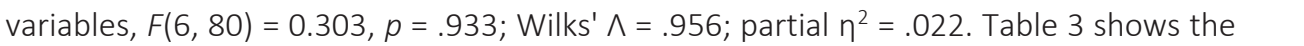
means and standard deviations on the questionnaire measures.

A further univariate analysis showed a statistically significant difference among the number of sessions on attitudes toward condom use, $F(2,42)=3 . \beta<2.095$; ; partiaf f $^{2}$ .127, and attitudes toward refraining from risky sexual practices, $F(2,42)=5$. 4 < 2.05 ; partial $\eta^{2}=.196$. Multiple comparisons showed that participants who finished five sessions 
had significantly stronger positive attitudes toward condom use $(p<.05)$ compared to participants who only finished one session, with a mean increase of the difference score (postintervention measurement minus pre-intervention measurement) of $0.40,95 \% \mathrm{Cl}[0.02$, 1.24]. No significant difference was observed between one and three sessions ( $p=.70$ ) or between three and five sessions ( $p=.20$ ). Interestingly, participants whönished three sessions had significantly less positive attitudes toward refraining from risky sex practices $(p<.05)$ compared to participants who only finished one session, with a mean decrease of the difference score (post-intervention measurement minus pre-intervention measurement) of $-0.27,95 \% \mathrm{Cl}[-1.59,-0.21]$. No significant difference was observed between one and five sessions ( $p=.15$ ) or between three and five session $\beta$ \& 40 ).

For perceived norms associated with refraining from risky sex practices, univariate analysis showed a statistically significant difference among the number of sessions, $F(2$, 42 ) = $9.4 p 3<10,01$; partiß $=n .310$. A multiple comparison analysis revealed that participants who finished three sessions reported a significantly stronger normative influence toward refraining from risky sex practices $(p<.05)$ compared to participants who only finished one session, with a mean increase of the difference score (post-intervention measurement minus pre-intervention measurement) of $0.50,95 \% \mathrm{Cl}[0.09,1.45]$. Furthermore, participants who finished five sessions also reported a significantly stronger normative influence toward refraining from risky sex practices $(p<.001)$ compared to participants who only finished one session, with a mean increase of the difference score (post-intervention measurement minus pre-intervention measurement) of $0.30,95 \% \mathrm{Cl}[0.52,1.88]$. No significant difference was observed between three and five sessions ( $p=.27$ ).

Lastly, univariate analysis showed a statistically significant difference in intention to ask a new sex partner about his or her sexual health status, $F(2,42)=4 . \$$. 4 ; partial $\eta^{2}=.183$, andntention to refrain from risky sex practices, $F(2,42)=7 . \overline{5} 53$, .001 ; partia $¥ \eta 270$, depending on the number of sessions. A multiple comparison analysis showed that participants who finished three sessions reported a significantly weaker intention to ask a new sex partner about his or her health status $(p<.05)$ compared to participants who only finished one session, with a mean decrease of the difference score (post-intervention measurement minus pre-intervention measurement) of $-1.60,95 \% \mathrm{Cl}$ $[-3.34,-0.26]$. No significant difference was observed between one and five sessions $(p=$ .06 ) and between three and five sessions ( $p=.90$ ). Also, participants who finished five sessions reported a significantly weaker intention toward refraining from risky sex practices $(p<.001)$ compared to participants who only finished one session, with a mean decrease of the difference score (post-intervention measurement minus pre-intervention measurement) of $-1.00,95 \% \mathrm{Cl}[-4.33,-1.00]$. Participants who finished five sessions also reported a significantly weaker intention toward refraining from risky sex practices ( $p<$ .05) compared to participants who only finished three sessions, with a mean decrease of the difference score (post-intervention measurement minus pre-intervention measurement) of $-0.84,95 \% \mathrm{Cl}[-3.33,0.00]$. No significant difference was observed between one and three sessions $(p=.32$ ). 
Table 3. Means (M) and standard deviations (SD) of questionnaire items ( $P B C=$ perceived behavioral control) in different conditions: evaluative conditioning active $\left(E C_{a}\right)$, evaluative conditioning inactive $\left(E C_{m}\right)$, behavioral inhibition training active $\left(\left.B\right|_{a}\right)$, behavioral inhibition training inactive $\left(\left.B\right|_{m}\right)$, working memory training active $\left(W M_{a}\right)$, and working memory training inactive $\left(\mathrm{WM}_{\mathrm{m}}\right)$.

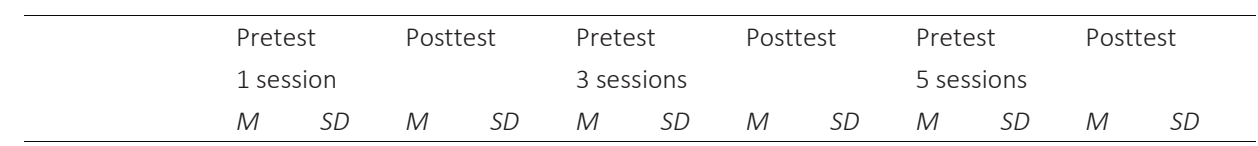

\section{Condom use}

Attitudes

\begin{tabular}{|c|c|c|c|c|c|c|c|c|c|c|c|c|}
\hline$E C_{a}, B I_{a}, W M_{a}$ & 4.07 & 0.258 & 3.87 & 0.581 & 4.03 & 1.060 & 4.03 & 1.125 & 3.83 & 1.029 & 4.27 & 0.372 \\
\hline$E C_{a}, B l_{a}, W M_{m}$ & 4.3 & 0.978 & 4.17 & 1.063 & 4.03 & 0.352 & 4.13 & 0.611 & 4.5 & 0.732 & 4.4 & 0.712 \\
\hline$E C_{a}, B I_{m}, W M_{m}$ & 4.3 & 0.414 & 4.33 & 0.617 & 4.13 & 0.442 & 4.07 & 0.372 & 4.3 & 0.621 & 4.33 & 0.588 \\
\hline$E C_{m}, B l_{a}, W M_{m}$ & 4.17 & 0.939 & 4.13 & .743 & 4.07 & .258 & 4.23 & .704 & 4.07 & 0.417 & 4.47 & .834 \\
\hline$E C_{m}, B I_{m}, W M_{m}$ & - & - & - & - & - & - & - & 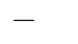 & 4.13 & 0.297 & 4.1 & 0.806 \\
\hline \multicolumn{13}{|l|}{ Norms } \\
\hline$E C_{a}, B I_{a}, W M_{a}$ & 3.73 & 0.904 & 3.87 & 0.229 & 3.53 & 1.343 & 3.57 & 0.799 & 3.77 & 0.863 & 3.83 & 0.45 \\
\hline$E C_{a}, B l_{a}, W M_{m}$ & 4.2 & 1.360 & 4.17 & 0.588 & 3.87 & 0.821 & 4.03 & 0.719 & 3.67 & 0.923 & 3.7 & 0.676 \\
\hline$E C_{a}, B I_{m}, W M_{m}$ & 3.3 & 1.014 & 3.7 & 0.882 & 3.6 & 0.604 & 3.36 & 0.806 & 3.47 & .0834 & 3.93 & 0.678 \\
\hline$E C_{m}, B l_{a}, W M_{m}$ & 4.03 & 1.06 & 3.8 & 0.702 & 3.37 & 0.972 & 3.77 & 0.623 & 3.33 & 0.976 & 3.63 & 0.855 \\
\hline$E C_{m}, B I_{m}, W M_{m}$ & - & - & - & - & - & - & - & - & 3.7 & 0.592 & 4.1 & 0.712 \\
\hline \multicolumn{13}{|l|}{$P B C$} \\
\hline $\mathrm{EC}_{a}, \mathrm{Bl}_{\mathrm{a}}, \mathrm{WM}_{\mathrm{a}}$ & 1.8 & 1.373 & 1.47 & 1.552 & 2.33 & 1.839 & 1.27 & 1.907 & 2.13 & 1.642 & 1.67 & 1.291 \\
\hline $\mathrm{EC}_{\mathrm{a}}, \mathrm{Bl}_{\mathrm{a}}, \mathrm{WM}_{\mathrm{m}}$ & 2.93 & 1.710 & 1.87 & 1.727 & 2.33 & 1.718 & 1.73 & 1.1 & 2.4 & 1.724 & 1.6 & 1.242 \\
\hline$E C_{a}, B I_{m}, W M_{m}$ & 1.53 & 1.06 & 1.47 & 1.302 & 1.53 & 0.743 & 1.33 & 1.047 & 1.87 & 1.727 & 1.67 & 1.397 \\
\hline$E C_{m}, B l_{a}, W M_{m}$ & 1.67 & 0.9 & 1.27 & 1.907 & 1.53 & 1.06 & 1.2 & 0.561 & 1.93 & 1.486 & 1.73 & 1.580 \\
\hline$E C_{m}, B I_{m}, W M_{m}$ & - & - & - & - & - & - & - & - & 2.07 & 1.335 & 2.47 & 1.246 \\
\hline \multicolumn{13}{|l|}{ Intentions } \\
\hline$E C_{a}, B I_{a}, W M_{a}$ & 6.07 & $1 . €$ & 6.8 & 51 & 6.07 & 1 & 6.3 & 1.935 & 6.07 & 163 & 6.53 & 0.915 \\
\hline$E C_{a}, B l_{a}, W M_{m}$ & 5.4 & 1.844 & 5.33 & 1.839 & 5.73 & 1.387 & 6.03 & 1.447 & 5.63 & 2.1 & 6.07 & 1.486 \\
\hline$E C_{a}, B l_{m}, W M_{m}$ & 6.13 & 1.767 & 6.4 & 1.549 & 6.33 & 1.291 & 6.73 & 0.458 & 6.67 & 0.816 & 6.8 & 0.561 \\
\hline$E C_{m}, B l_{a}, W M_{m}$ & 6.0 & 1.732 & 6.4 & 1.121 & 6.73 & 0.594 & 6.4 & 1.121 & 5.93 & 1.624 & 6.2 & 1.568 \\
\hline$E C_{m}, B I_{m}, W M_{m}$ & - & - & - & - & - & - & - & - & 5.6 & 1.404 & 5.73 & 1.486 \\
\hline
\end{tabular}

\section{Health status}

\section{Attitudes}

\begin{tabular}{lllllllllllll}
$E C_{a}, B I_{a}, W M_{a}$ & 4.8 & 1.032 & 5.07 & 0.961 & 4.37 & 1.217 & 4.83 & 1.410 & 4.67 & 0.880 & 4.73 & 0.594 \\
$E C_{a}, B l_{a}, W M_{m}$ & 4.73 & 1.033 & 4.53 & 0.935 & 4.3 & 0.797 & 4.81 & 0.972 & 4.87 & 1.202 & 4.9 & 1.153 \\
$E C_{a}, B I_{m}, W M_{m}$ & 5.2 & 1.131 & 5.17 & 1.047 & 5.07 & 0.942 & 5.03 & 0.855 & 5.47 & 0.935 & 5.47 & 1.043 \\
$E C_{m}, B l_{a}, W M_{m}$ & 4.97 & 1.232 & 4.87 & 0.743 & 4.77 & 1.015 & 5.2 & 0.941 & 4.4 & 1.039 & 4.87 & 0.884 \\
$E C_{m}, B I_{m}, W M_{m}$ & - & - & - & - & - & - & - & - & 5.13 & 0.812 & 4.73 & 1.294 \\
$N o r m s$ & & & & & & & & & & & & \\
$E C_{a}, B l_{a}, W M_{a}$ & 3.87 & 0.812 & 3.47 & 0.55 & 3.1 & 1.072 & 3.3 & 1.279 & 3.53 & 0.855 & 3.6 & 0.660 \\
$E C_{a}, B l_{a}, W M_{m}$ & 3.37 & 1.288 & 3.83 & 1.012 & 3.4 & 1.137 & 3.37 & 0.611 & 3.77 & 0.998 & 3.23 & 0.753 \\
$E C_{a}, B I_{m}, W M_{m}$ & 2.9 & 1.168 & 3.27 & 1.043 & 3.1 & 0.761 & 3.43 & 0.594 & 3.0 & 0.845 & 3.07 & 1.132 \\
$E C_{m}, B I_{a}, W M_{m}$ & 3.23 & 1.083 & 3.3 & 0.96 & 3.23 & 1.4 & 3.4 & 0.66 & 3.2 & 0.941 & 3.2 & 0.819 \\
$E C_{m}, B I_{m}, W M_{m}$ & - & - & - & - & - & - & - & - & 3.17 & 0.859 & 3.5 & 0.655 \\
\hline
\end{tabular}




\begin{tabular}{|c|c|c|c|c|c|c|c|c|c|c|c|c|}
\hline & \multicolumn{2}{|c|}{$\begin{array}{l}\text { Pretest } \\
1 \text { session }\end{array}$} & \multicolumn{2}{|c|}{ Posttest } & \multicolumn{2}{|c|}{$\begin{array}{l}\text { Pretest } \\
3 \text { sessions }\end{array}$} & \multicolumn{2}{|c|}{ Posttest } & \multicolumn{2}{|c|}{$\begin{array}{l}\text { Pretest } \\
5 \text { sessions }\end{array}$} & \multicolumn{2}{|c|}{ Posttest } \\
\hline & $M$ & $S D$ & $M$ & $S D$ & $M$ & $S D$ & $M$ & $S D$ & $M$ & $S D$ & $M$ & $S D$ \\
\hline \multicolumn{13}{|l|}{$P B C$} \\
\hline$E C_{a}, B_{a}, W M_{a}$ & 3.67 & 1.799 & 2.91 & 2.028 & 3.53 & 2.031 & 2.93 & 1.668 & 3.53 & 2.031 & 2.73 & 1.438 \\
\hline $\mathrm{EC}_{\mathrm{a}}, \mathrm{Bl}_{\mathrm{a}}, \mathrm{WM}_{\mathrm{m}}$ & 3.27 & 1.792 & 2.93 & 1.58 & 2.87 & 1.187 & 2.2 & 1.207 & 3.33 & 1.718 & 3.47 & 1.125 \\
\hline $\mathrm{EC}_{\mathrm{a}}, \mathrm{B} \mathrm{I}_{\mathrm{m}}, \mathrm{WM}_{\mathrm{m}}$ & 2.27 & 1.280 & 3.20 & 2.031 & 2.13 & 1.06 & 3.38 & 1.447 & 4.13 & 1.727 & 3.87 & 1.474 \\
\hline $\mathrm{EC}_{\mathrm{m}}, \mathrm{Bl}_{\mathrm{a}}, \mathrm{WM}_{\mathrm{m}}$ & 3.0 & 1.512 & 2.73 & 2.017 & 2.6 & 1.454 & 2.0 & 1.363 & 2.47 & 1.727 & 2.6 & 1.882 \\
\hline $\begin{array}{l}\mathrm{EC}_{\mathrm{m}}, \mathrm{B} \mathrm{I}_{\mathrm{m}}, \mathrm{WM}_{\mathrm{m}} \\
\text { Intentions }\end{array}$ & - & - & - & - & - & - & - & - & 3.53 & 1.846 & 3.13 & 1.457 \\
\hline$E C_{a}, B I_{a}, W M_{a}$ & 4.0 & 1.964 & 5.07 & 1.995 & 3.8 & 1.859 & 3.47 & 1.624 & 2.87 & 1.959 & 3.87 & 1.727 \\
\hline$E C_{a}, B I_{a}, W M_{m}$ & 4.0 & 1.964 & 3.93 & 1.624 & 3.67 & 2.225 & 3.53 & 1.807 & 4.27 & 1.792 & 3.07 & 1.534 \\
\hline$E C_{a}, B I_{m}, W M_{m}$ & 3.8 & 2.077 & 4.07 & 2.07 & 3.13 & 2.1 & 3.0 & 1.944 & 4.27 & 1.87 & 3.81 & 1.944 \\
\hline$E C_{m}, B l_{a}, W M_{m}$ & 3.53 & 2.295 & 3.6 & 1.844 & 3.93 & 2.251 & 3.0 & 1.773 & 3.87 & 2.503 & 3.73 & 3.187 \\
\hline$E C_{m}, B I_{m}, W M_{m}$ & - & - & - & - & - & - & - & - & 3.93 & 1.792 & 4.13 & 2.264 \\
\hline \multicolumn{13}{|l|}{ Sexual practice } \\
\hline \multicolumn{13}{|l|}{ Attitudes } \\
\hline $\mathrm{EC}_{\mathrm{a}}, \mathrm{Bl}_{\mathrm{a}}, \mathrm{WM}_{\mathrm{a}}$ & 4.23 & 0.530 & 4.17 & 0.338 & 3.60 & 1.039 & 3.9 & 1.029 & 4.2 & 0.528 & 4.4 & 0.573 \\
\hline $\mathrm{EC}_{\mathrm{a}}, \mathrm{Bl}_{\mathrm{a}}, \mathrm{WM}_{\mathrm{m}}$ & 4.03 & 1.125 & 4.3 & 0.941 & 4.0 & 0.5 & 3.73 & 0.799 & 4.03 & 0.719 & 4.1 & 1.105 \\
\hline $\mathrm{EC}_{\mathrm{a}}, \mathrm{B} \mathrm{I}_{\mathrm{m}}, \mathrm{WM}_{\mathrm{m}}$ & 4.27 & 0.594 & 4.03 & 0.935 & 4.17 & 0.488 & 4.07 & 0.372 & 4.2 & 0.316 & 3.97 & 0.743 \\
\hline $\mathrm{EC}_{\mathrm{m}}, \mathrm{Bl}_{\mathrm{a}}, \mathrm{WM}_{\mathrm{m}}$ & 4.13 & 0.516 & 4.17 & .0309 & 3.87 & 0.855 & 3.83 & 0.935 & 3.83 & 0.748 & 3.97 & 0.297 \\
\hline$E C_{m}, B I_{m}, W M_{m}$ & - & - & - & - & - & - & - & - & 3.77 & 0.821 & 4.33 & 0.673 \\
\hline \multicolumn{13}{|l|}{ Norms } \\
\hline$E C_{a}, B l_{a}, W M_{a}$ & 3.5 & 0.779 & 3.67 & 0.556 & 3.77 & 0.98 & 4.17 & 0.88 & 4.20 & 0.797 & 3.97 & 0.772 \\
\hline$E C_{a}, B l_{a}, W M_{m}$ & 3.87 & 1.077 & 3.93 & 0.623 & 3.87 & 1.026 & 3.6 & 1.137 & 3.53 & 0.79 & 3.9 & 0.660 \\
\hline$E C_{a}, B I_{m}, W M_{m}$ & 3.77 & 1.067 & 4.07 & 1.223 & 3.8 & 1.424 & 3.73 & 0.753 & 3.27 & 0.842 & 4.17 & 0.743 \\
\hline$E C_{m}, B l_{a}, W M_{m}$ & 3.7 & 0.676 & 4.17 & 0.748 & 3.57 & 0.776 & 3.83 & 0.994 & 3.23 & 1.116 & 3.6 & 1.121 \\
\hline$E C_{m}, B I_{m}, W M_{m}$ & - & - & - & - & - & - & - & - & 4.07 & 0.623 & 3.83 & 0.699 \\
\hline \multicolumn{13}{|l|}{$P B C$} \\
\hline $\mathrm{EC}_{\mathrm{a}}, \mathrm{Bl}_{\mathrm{a}}, \mathrm{WM}_{\mathrm{a}}$ & 2.73 & 1.534 & 2.33 & 1.877 & 3.27 & 1.792 & 3.47 & 1.685 & 3.0 & 1.732 & 3.21 & 1.656 \\
\hline $\mathrm{EC}_{\mathrm{a}}, \mathrm{Bl}_{\mathrm{a}}, \mathrm{WM}_{\mathrm{m}}$ & 3.47 & 1.959 & 3.27 & 1.534 & 2.13 & 1.457 & 2.53 & 1.407 & 3.13 & 1.922 & 3.27 & 1.751 \\
\hline $\mathrm{EC}_{\mathrm{a}}, \mathrm{BI} \mathrm{m}, \mathrm{WM}_{\mathrm{m}}$ & 2.4 & 1.549 & 2.4 & 2.028 & 2.73 & 1.71 & 2.8 & 1.302 & 1.8 & 1.656 & 1.87 & 1.821 \\
\hline$E C_{m}, \mathrm{Bl}_{a}, \mathrm{WM}_{\mathrm{m}}$ & 2.93 & 1.534 & 2.2 & 0.775 & 2.67 & 2.093 & 2.6 & 2.063 & 1.87 & 1.356 & 2.53 & 2.167 \\
\hline$E C_{m}, B I_{m}, W M m$ & - & - & - & - & - & - & - & - & 2.53 & 1.187 & 2.4 & 1.121 \\
\hline \multicolumn{13}{|l|}{ Intentions } \\
\hline$E C_{a}, B l_{a}, W M_{a}$ & 3.2 & 1.935 & 4.13 & 2.134 & 2.53 & 1.767 & 3.97 & 1.767 & 2.73 & 1.792 & 3.13 & 1.676 \\
\hline$E C_{a}, B l_{a}, W M_{m}$ & 3.41 & 1.993 & 3.07 & 1.751 & 2.73 & 1.668 & 2.67 & 2.127 & 3.6 & 2.063 & 2.93 & 2.052 \\
\hline$E C_{a}, B I_{m}, W M_{m}$ & 2.21 & 1.935 & 2.0 & 1.254 & 2.67 & 1.454 & 2.93 & 2.251 & 3.07 & 2.052 & 2.83 & 1.877 \\
\hline$E C_{m}, B l_{a}, W M_{m}$ & 2.6 & 1.502 & 2.67 & 0.9 & 3.47 & 2.031 & 3.87 & 1.995 & 3.0 & 2.07 & 3.0 & 2.138 \\
\hline$E C_{m}, B I_{m}, W M_{m}$ & - & - & - & - & - & - & - & - & 2.53 & 1.246 & 3.13 & 1.506 \\
\hline
\end{tabular}

\section{SC-IAT data}

A MANOVA showed no statistically significant difference between the number of sessions on the implicit attitudes toward condom use, $F(4,82)=4.99311,3$; Wilks' $\Lambda=.820$; partial $\eta^{2}=.180$; on the implicit attitudes toward asking about the current sexhealth 
status of a new partner, $F(4,82)=0.8=2.5613$; Wilks' $\Lambda=.924$; partiøl $\$$ \%; and on the implicit attitudes toward refraining from risky sex practices, $F(4,82)=0.079$,

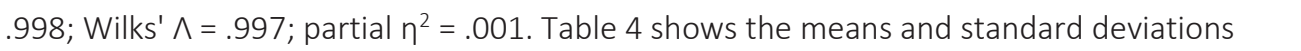
of the D600 scores.

Table 4. Means (M) and standard deviations (SD) of D600 data in different conditions: evaluative conditioning active $\left(E C_{a}\right)$, evaluative conditioning inactive $\left(E C_{m}\right)$, behavioral inhibition training active $\left(B l_{a}\right)$, behavioral inhibition training inactive $\left(B I_{m}\right)$, working memory training active $\left(W M_{a}\right)$, and working memory training inactive $(\mathrm{WM})$.

\begin{tabular}{|c|c|c|c|c|c|c|c|c|c|c|c|c|}
\hline & \multirow{2}{*}{\multicolumn{2}{|c|}{$\frac{\text { Pretest }}{1 \text { session }}$}} & \multicolumn{2}{|c|}{ Posttest } & \multirow{2}{*}{\multicolumn{2}{|c|}{$\frac{\text { Pretest }}{3 \text { sessions }}$}} & \multicolumn{2}{|c|}{ Posttest } & \multirow{2}{*}{\multicolumn{2}{|c|}{$\frac{\text { Pretest }}{5 \text { sessions }}$}} & \multicolumn{2}{|c|}{ Posttest } \\
\hline & & & & & & & & & & & & \\
\hline & $M$ & SD & M & SD & $M$ & SD & M & SD & $M$ & SD & M & SD \\
\hline \multicolumn{13}{|l|}{ Condom use } \\
\hline ECa, Bla, WMa & 0.108 & 0.227 & 0.096 & 0.169 & 0.046 & 0.133 & 0.043 & 0.079 & -0.035 & 0.167 & 0.061 & 0.151 \\
\hline ECa, Bla, WMm & 0.014 & 0.143 & 0.048 & 0.158 & 0.025 & 0.122 & 0.054 & 0.179 & 0.040 & 0.143 & -0.027 & 0.152 \\
\hline ECa, BIm, WMm & 0.102 & 0.235 & 0.056 & 0.185 & 0.007 & 0.263 & -0.018 & 0.229 & 0.039 & 0.150 & 0.034 & 0.157 \\
\hline ECm, Bla, WMm & 0.086 & 0.139 & 0.083 & 0.169 & 0.129 & 0.114 & 0.044 & 0.135 & 0.058 & 0.115 & 0.087 & 0.177 \\
\hline $\mathrm{ECm}, \mathrm{BIm}, \mathrm{WMm}$ & - & - & - & - & - & - & - & - & 0.063 & 0.164 & 0.103 & 0.149 \\
\hline \multicolumn{13}{|l|}{ Health status } \\
\hline ECa, Bla, WMa & -0.019 & 0.261 & -0.019 & 0.235 & 0.039 & 0.104 & 0.065 & 0.151 & 0.035 & 0.161 & -0.009 & 0.119 \\
\hline ECa, Bla, WMm & -0.003 & 0.093 & 0.038 & 0.204 & 0.068 & 0.117 & 0.016 & 0.134 & 0.063 & 0.162 & 0.096 & 0.117 \\
\hline ECa, BIm, WMm & 0.051 & 0.136 & -0.007 & 0.174 & -0.056 & 0.366 & 0.060 & 0.340 & 0.025 & 0.097 & 0.049 & 0.183 \\
\hline ECm, Bla, WMm & 0.074 & 0.208 & 0.065 & 0.161 & 0.067 & 0.070 & -0.028 & 0.184 & 0.033 & 0.127 & 0.057 & 0.120 \\
\hline $\mathrm{ECm}, \mathrm{BIm}, \mathrm{WMm}$ & - & - & - & - & - & - & - & - & 0.034 & 0.110 & 0.037 & 0.104 \\
\hline \multicolumn{13}{|l|}{ Sexual practice } \\
\hline ECa, Bla, WMa & 0.041 & 0.145 & -0.008 & 0.146 & 0.026 & 0.148 & 0.001 & 0.097 & 0.031 & 0.153 & -0.003 & 0.169 \\
\hline ECa, Bla, WMm & 0.067 & 0.137 & 0.039 & 0.171 & 0.000 & 0.112 & 0.043 & 0.141 & -0.011 & 0.125 & 0.043 & 0.157 \\
\hline ECa, BIm, WMm & 0.065 & 0.163 & -0.013 & 0.186 & -0.120 & 0.183 & 0.064 & 0.225 & 0.321 & 0.086 & 0.017 & 0.109 \\
\hline ECm, Bla, WMm & 0.045 & 0.214 & 0.029 & 0.127 & 0.042 & 0.150 & 0.036 & 0.133 & -0.011 & 0.155 & 0.027 & 0.127 \\
\hline ECm, BIm, WMm & - & - & - & - & - & - & - & - & -0.011 & 0.205 & 0.054 & 0.145 \\
\hline
\end{tabular}

Active evaluative conditioning, active behavioral inhibition, mock working memory training

\section{Questionnaire data}

With only two components active (evaluative conditioning and behavioral inhibition training) and a mock working memory training condition, multivariate analysis showed no significant differences between the number of sessions on combined attitudes, $F(6$, 80 ) = $1.0 p 3=94,06$; Wilks' $\Lambda=.861$; pátiøl $\nabla 2$; on combined perceived norms,

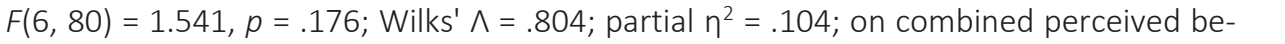
havioral control variables, $F(6,80)=0.8=8.551,0 ;$ Wilks' $^{\prime} \Lambda=.879 ;$ pa $^{2}$ atial 62 ; and on combined intentions, $F(6,80)=2 . \$ 7.0 B 5,4 ;$ Wilks' $\Lambda=.739$; paftial 40 . 


\section{SC-IAT data}

For this condition, a MANOVA also showed no statistically significant difference between the number of sessions on implicit attitudes toward condom use, $F(4,82)=0 . \$ 47$, $=.500$; Wilks' $\Lambda=.922$; partiaD $\mathrm{nA} 0$; on implicit attitudes toward asking abdae current sexual health status of a new partner, $F(4,82)=1.73 .7 .2487$; Wilks' $\Lambda=.886$; partial $\eta^{2}=.059$; or on implicit attitudes toward refraining from risky sex practickels4,

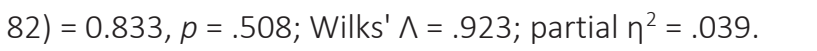

\section{Active evaluative conditioning, mock behavioral inhibition, mock working memory training}

\section{Questionnaire data}

With only one component active (evaluative conditioning) and a mock behavioral inhibition training and a mock working memory training condition, there was a statistically significant difference between the number of sessions on combined perceived behavioral control variables, $F(6,80)=3.85 .075$, ; Wilks' $\Lambda=.602$; partiaRn2 4 ; and the combined intentions, $F(6,80)=2 . \Phi 4.65 ;$ Wilks' $\Lambda=966$; partiaP 1.166 ; but not on

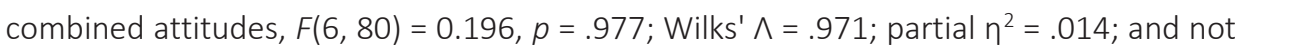
on combined perceived norms, $F(6,80)=1 . \beta=7.263,4 ;$ Wilks' $\Lambda=.822$; páftial 1 自 4 .

A further univariate analysis showed a statistically significant difference among the number of sessions in perceived behavioral control toward asking a new sex partner about his or her sexual health status, $F(2,42)=6$. 102.05 ; partiaf i 242 daperceived behavioral control toward refraining from risky sexual practices, $F\left(\begin{array}{ll}2 & 4 \\ 2\end{array}\right)=3.934$, $p<.05$; partiafif.1 58 .

A multiple comparison analysis showed that participants who finished five sessions reported a significantly higher perceived behavioral control related to asking a new sex partner about his or her health status $(p<.05)$ compared to participants who only finished one session, with a mean increase of the difference score (post-intervention measurement minus pre-intervention measurement) of $0.67,95 \% \mathrm{Cl}[0.85,4.22]$. No significant difference was observed between one and three sessions ( $p=.11$ ) and between three and five sessions ( $p=.24$ ). Furthermore, participants who finished five sessions reported a significantly weaker perceived behavioral control toward refraining from risky sex practices $(p<.05)$ compared to participants who only finished three sessions, with a mean decrease of the difference score (post-intervention measurement minus pre-intervention measurement) of $-0.93,95 \% \mathrm{Cl}[-3.48,-0.25]$. No significant difference was observed between one and three sessions ( $p=.40$ ) and between one and five sessions $=.30$ ).

Regarding intentions, a univariate analysis revealed a statistically significant difference in intention toward asking a new sex partner about his or her sexual health status, $F(2,42)=4 . \bar{b} \varangle .065$; ; partiaf 7.183 , depending on the number of sessions. A multiple comparison analysis showed that participants who finished three sessions reported a significantly weaker intention to ask a new sex partner about his or her health status $(p<$ 
.05) compared to participants who only finished one session, with a mean decrease of the difference score (post-intervention measurement minus pre-intervention measurement) of $-1.07,95 \% \mathrm{Cl}[-3.13,-0.34]$. No significant difference was observed between one and five sessions ( $p=.55$ ) or between three and five session $p$ \&.13 ).

\section{SC-IAT data}

With evaluative conditioning as the only active component, a MANOVA showed a statistically significant difference between the number of sessions on implicit attitudes toward refraining from risky sex practices, $F(4,82)=3 . \bar{b} 5.05$; Wilks' $\Lambda=.715$; partial $\eta$ .155. There was no statistically significant difference on implicit attitudes toward condom

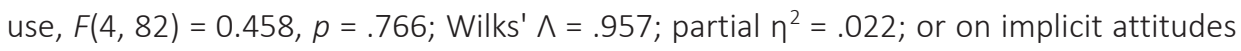
toward asking about the current sexual health status of a new partner, $F(4,82)=1.251$,

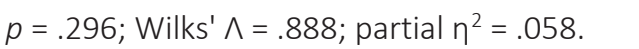

A multiple comparison analysis showed that participants who finished three sessions showed significantly higher D600 scores associated with refraining from risky sex practices $(p<.05)$ compared to participants who only finished one session, with a mean increase of the D600 score of $0.07,95 \% \mathrm{Cl}[0.05,0.31]$. Furthermore, participants who finished five sessions showed significantly lower D600 scores associated with refraining from risky sex practices $(p<.05)$ compared to participants who only finished three sessions, with a mean decrease of the $\mathrm{D} 600$ score of $0.04,95 \% \mathrm{Cl}[-0.28,-0.01]$. No significant difference was observed between one and five sessions ( $p=.99$ ).

\section{Active behavioral inhibition, mock evaluative conditioning, mock working memory training}

\section{Questionnaire data}

With only one component active (this time behavioral inhibition training) and a mock evaluative conditioning and a mock working memory training condition, there were no significant differences between the number of sessions on combined attitudes, $F(6,80)$ $=0.60 p 8=, 723$; Wilks' $\Lambda=.915$; parti. 144 ; on combined perceivecorms, $F(6$,

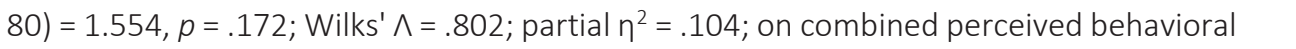
control variables, $F(6,80)=2.0=2.077,2$; Wilks' $\Lambda=.753$; pátial 1 , 2 ; or on com-

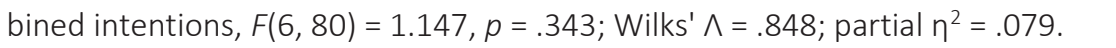

\section{SC-IAT data}

For this condition, a MANOVA showed no statistically significant difference between the number of sessions on implicit attitudes toward condom use, $F\left(\begin{array}{lll}4,8 & 2\end{array}\right)=1.95 .222,4$; Wilks' $\Lambda=.872$; partial=.0 66 ; on implicit attitudes toward asking about the current sexual health status of a new partner, $F(4,82)=1 . \rho=1.480,3$; Wilks' $\wedge=.908$; partial $\eta$

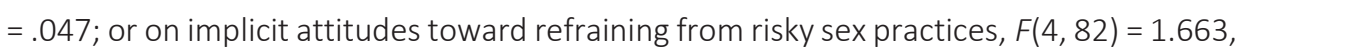

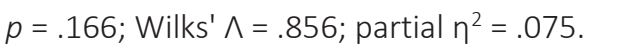


Mock behavioral inhibition, mock evaluative conditioning, mock working memory training

Questionnaire data

With only mock components, no significant difference between pre-intervention and post-intervention measurements could be detected after participants finished five sessions (all p's > .115).

\section{SC-IAT data}

An ANOVA detected no significant difference in D600 scores between pre- and post-

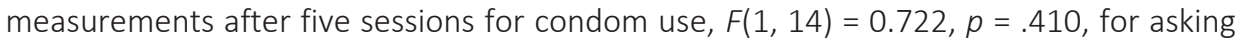
about the current health status of a new sex partner, $F(1,14)=0.0 € .945$, or for refraining from risky sex practices, $F(1,14)=1 . p=1.383,0$.

Active evaluative conditioning, active selective inhibition training, and active working memory training contrasted to mock conditions

Planned contrasts across all dosages of training (1, 3, and 5 gaming sessions combined) Planned contrasts showed that across all dosages of selective inhibition training perceived norms related to refraining from risky sex acts were significantly lower after training $(M=3.8 \quad S D,=0.191)$ compared to mock conditiahs (4 .0 $8 D,=0.433)$, a mean difference of $-0.17,97.5 \% \mathrm{Cl}[-1.89,-.55], F(1,14)=20 p 8<4032,5$, partiall=n.5 98 . Further, also for selective inhibition training, perceived behavior control related to asking a new sex partner about the current sexual health status was also significantly lower after training $(M=2.8 \Phi D,=0.387$ ) compared to mock conditidiss $(3.13 D,=0.667$, a mean difference of $-0.33,97.5 \% \mathrm{Cl}[-4.66,-.62], F(1,14)=10 p 7<7022,5$, partial=n .435. However, still for selective inhibition training, intentions to refrain from risky sex acts were significantly higher after training $(M=3.23 D,=0.646)$ compared to mock

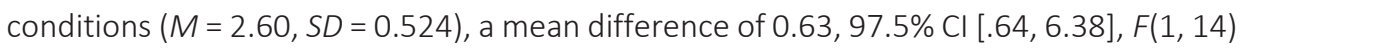
$=9.41 p 8,025$, partial=n.4 02 . Lastly, after working memory training, intentions to refrain from risky sex acts were significantly higher after training $\left(M=\begin{array}{lllll}3.9 & 8 D,=1 \\ \hline & 5 & 9\end{array}\right)$ compared to mock conditions $(M=2.76 D,=0.483)$, a mean difference of $1.21,97.5 \%$ $\mathrm{Cl}[4.24,11.86], F(1,14)=28 p 1<7042,5$, partial=n. 668 . Table 5 shows the means, standard deviation, mean differences, and contrast effects. 


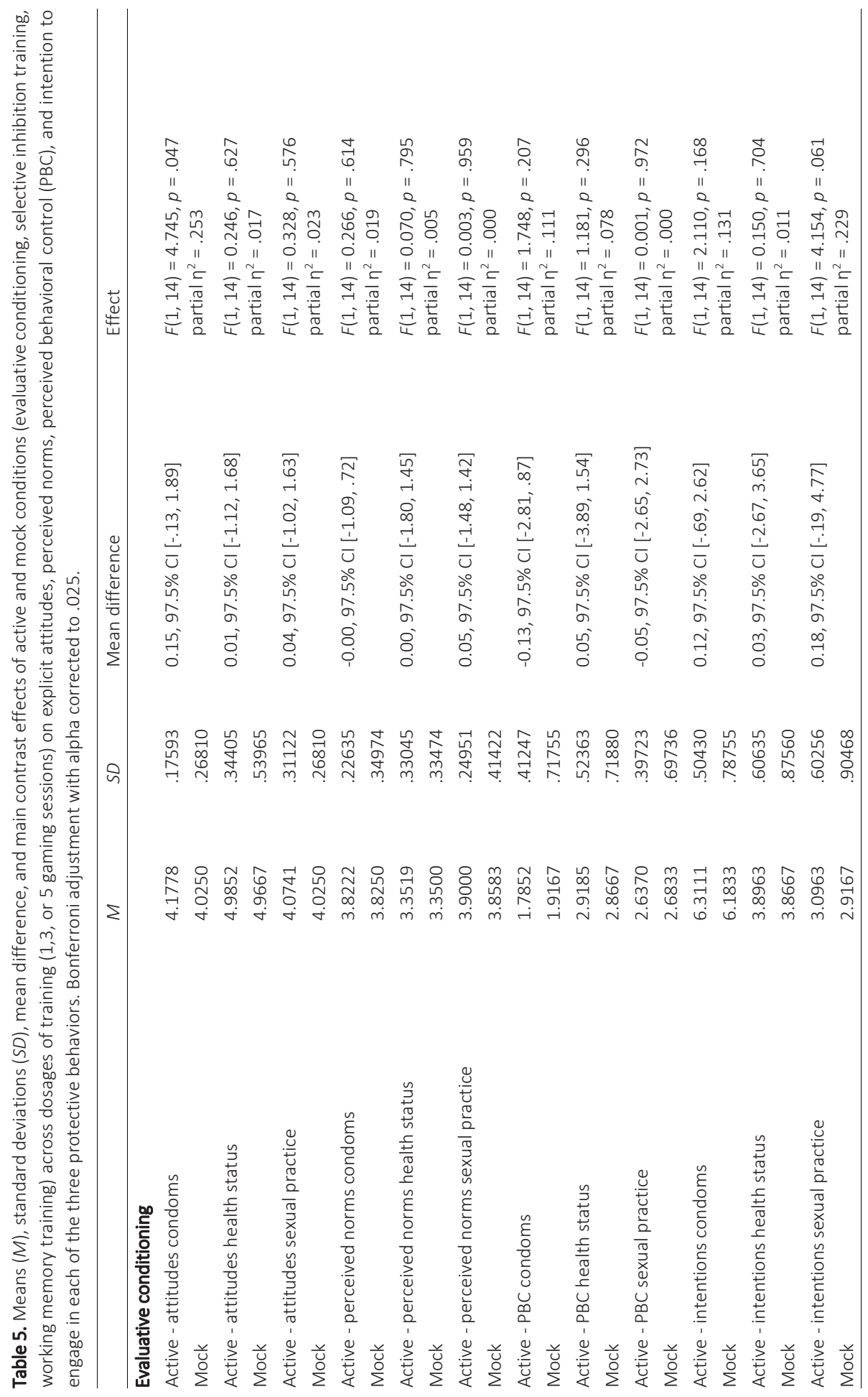




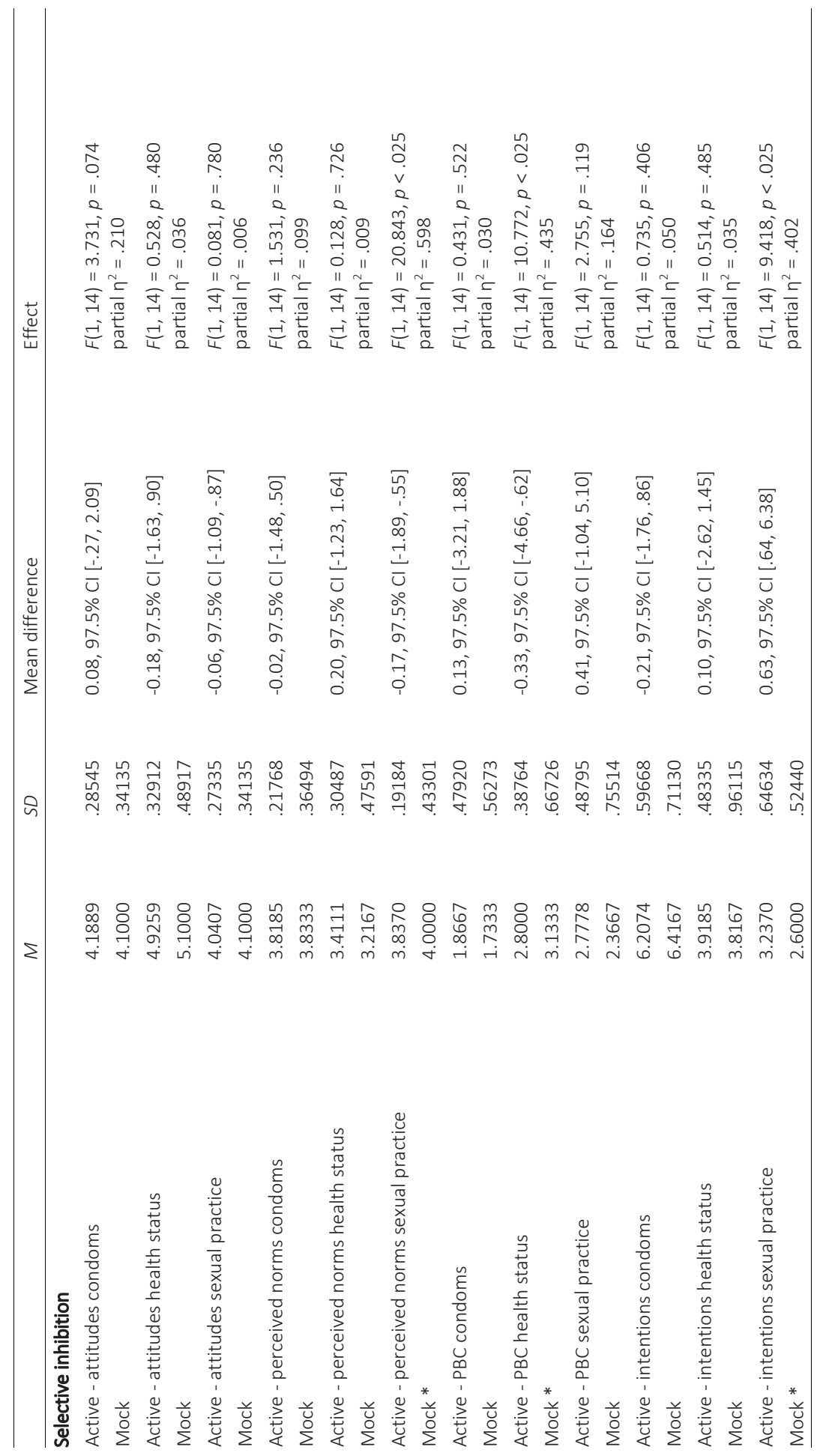




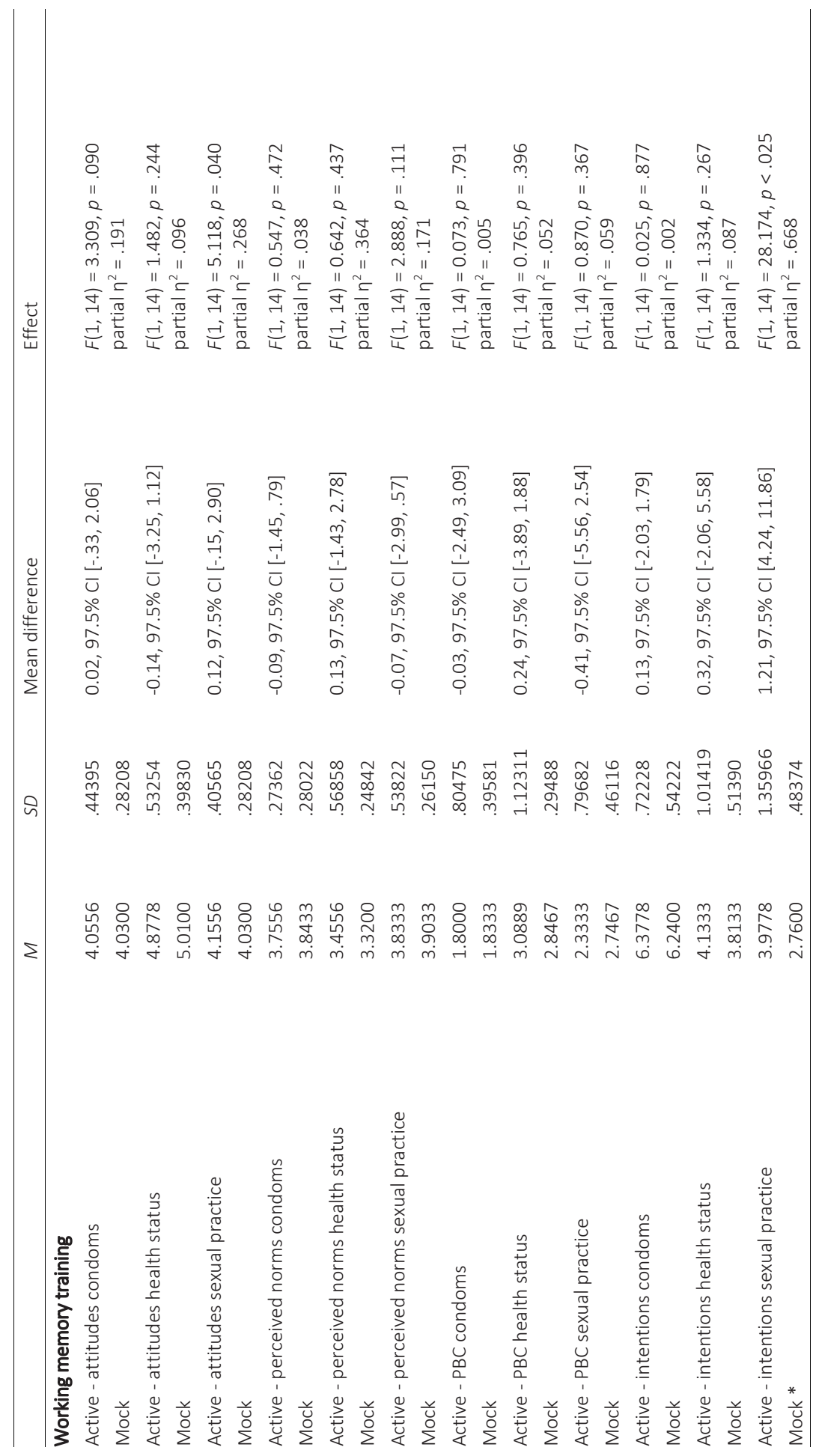


Planned contrasts by number of gaming sessions (1, 3, or 5)

Planned contrasts showed that after playing the game once when working memory training was active, attitudes towards refraining from risky sex acts $\left(M=3.9 \quad 8 D_{1}=\begin{array}{llll}0.3 & 3 & 8\end{array}\right)$ significantly decreased compared to the average of working memory mock conditions ( $M$ $=4.2 \Delta D,=0.367$ ), a mean difference of 0.30 , 9-.\$8.5-.085, IF (1, 14$)=7.999$,

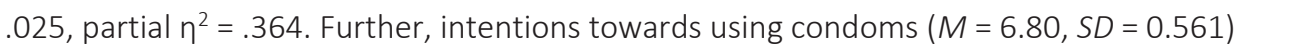
were significantly higher compared to the average of working memory mock conditions $(M=5.96 D,=0.789$ ), a mean difference of $0.84,97.5 \%$ CFF $B, 41,41) .3 \mathbb{1} \& .617$, $p<.025$, partial=ๆ.5 71 . Also, planned contrasts shehtenat after playing the game three times when working memory training was active, intentions towards asking about a partner's current sexual health $(M=3.4 \quad 8 D,=1.995)$ were significantly lower compared to the average of working memory mock conditions $\left(M=3.9 S D=\begin{array}{l}1 \\ 3\end{array} .079\right.$ ), a mean difference of $0.46,97.5 \% \mathrm{Cl}[.37,2.23], F(1,14)=6 . \bar{b} 8 . \$ 2,5$, partial=n.3 24 . Furthermore, after playing the game five times when working memory training was active,

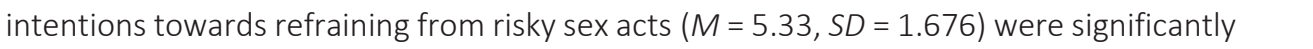
higher compared to the average of working memory mock conditions $(M=2.85 D$, = 0.900), a mean difference of $2.48,97.5 \% \mathrm{Cl}[1.20,3.76], F(1,14$ ) = $23 p \& .823$, partial $\eta^{2}=.628$. Further, planned contrastiowed that the average of perceived norms related to refraining from risky sex acts after playing the game five times with selective inhibition training active $\left(M=3.7 \quad 8 D,=\begin{array}{llll}0 & 5 & 6 & 5\end{array}\right)$ was significantly lower compared to the average of selective inhibition mock conditions $(M=4.1 \Phi D,=0.549$ ), a mean difference of 0.38 , $97.5 \% \mathrm{Cl}[-.71,-.03], F(1,14)=7 . \bar{p} 3.835$, partial=n.3 56 . Planned contrasts also showed that the average of intentions related to refraining from risky sex acts after playing the game five times with selective inhibition training active $\left(M=\begin{array}{lllll}3 & .7 & 50,=1 & 1 & 4\end{array}\right)$ was significantly higher compared to the average of selective inhibition mock conditions $(M=$

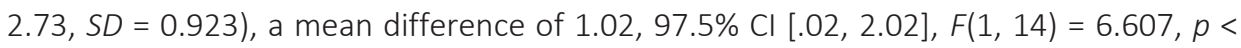
.025 , partiål=n.3 21 . No further significant differences were detected by contrasting active and mock conditions.

\section{Discussion}

In this study, we examined the effects of different combinations of active evaluative conditioning, selective response inhibition training, and working memory training components - presented in an online gaming environment - on explicit and implicit evaluations of three safer-sex behaviors with a new sex partner: using condoms, asking about the sexual health status, and refraining from risky sex practices). As expected, after five gaming sessions, no significant differences were observed in explicit and implicit determinants of behavior between pre- and post-gaming in the mock-only condition for all three behaviors of interest. 
Unexpectedly, there was no significant effect of selective RI training alone on explicit and implicit determinants of each of the three behaviors of interest. Only one contrast effect between active and mock conditions revealed a significantly more positive perceived behavioral control for asking a new sex partner about his or her current sexual health status after selective inhibition training. In fact, perceived norms to refrain from risky sex practices and intentions to engage in less risky sex practices during the next firsttime sexual intercourse with a new partner were significantly more positive in mock conditions contrasted to active conditions. Even if one could expect a transfer of selective RI training based on the central role that inhibition plays in several cognitive functions (Miyake \& Friedman, 2012), it did not occur here. This is in line with Enge et al. (2014), who found no conclusive evidence of transfer effects in young adults following stop-signal tasks in terms of non-trained cognitive functions. Furthermore, inhibitory control training using the stop-signal paradigm has been shown to only having a small effect on actual behavior (Allom et al., 2016). Moreover, a combination of active EC and selective RI training did not result in any significant pre-post-gaming differences in explicit and implicit variables, independent of the number of gaming sessions. We expected that not responding to an unsafe-sex cue in the SST would cause a devaluation of that cue (Chen, Veling, Dijksterhuis, \& Holland, 2016), especially if unsafe-sex decisions are conditioned to be disliked during the same gaming intervention. Unfortunately, the reasons why this effect did not occur in the selective RI active conditions remain unclear. As stated in a metaanalysis by Allom et al. (2016), there is no direct evidence that longer training sessions are more beneficial in influencing health behavior. In fact, selective inhibition was shown to have an effect on automatic associations, even after a single training session (Houben, Havermans, Nederkoorn, \& Jansen, 2012; Houben, Nederkoorn, Wiers, \& Jansen, 2011). This suggests that selective inhibition, instead of being classified as an executive function, could more likely be considered a form of cognitive bias modification (CBM), as suggested by Wiers (2018).

Only the condition with active EC resulted in a significant difference in implicit attitudes toward less risky sex practices. However, while attitudes towards refraining from risky sex practices became more positive after three gaming sessions (but still remained weak), initial positive implicit attitudes were significantly reduced for participants who played the game five times. These effects on an implicit level were, however, not observed on the measures of explicit determinants of behavior. Here, a reduction in PBC related to less risky sex practices and in intention toward asking a partner about his or her current sexual health status was observed for both three and five gaming sessions. Only PBC related to asking about the current sexual health status of a partner was significantly increased after five gaming sessions compared to only one session. These results may reflect the resilience of conditioned implicit attitudes and certain explicit behavioral determinants as, for instance, observed by Sweldens, Van Osselaer, and Janiszewski (2010). However, the mixed results we obtained in this condition do not allow us to draw even preliminary conclusions about the effectiveness of EC alone. 
Finally, the condition with all three components in active mode revealed several significant differences between pre- and post-gaming measurements in explicit, but not in implicit behavioral determinants. The normative influence on refraining from risky sex practices became more positive after three gaming sessions compared to only one session, and after five sessions compared to three sessions. Also, the explicit attitudes toward condom use were more positive after five gaming sessions compared to one session, whereas attitudes toward less risky sex practices became less positive after three gaming sessions compared to one session. However, most importantly, the intention to ask a new partner about his or her current sexual health status was weaker after three gaming sessions. Moreover, the intention to refrain from less risky sex practices was weaker after three and even weaker after five gaming sessions. Furthermore, the significant implicit and explicit effects observed in the EC-only condition were not observed in the EC and RI training condition, as this condition yielded no significant differences across gaming sessions at all. Only when WM training was added to EC and RI training, so that all three interventions were active, were there several significant differences, but mainly not in the direction that was predicted. To the contrary, the RAA variable intention (which is seen as the main predictor of actual behavior) was weaker for two behaviors after the intervention - also corroborated by significant contrast effects that show a weaker intention to engage in less risky sex practices after the intervention. The factors contributing to these effects in terms of transfer are unclear, as it has been shown that transfer of WM training, especially to reasoning, is inconsistent (e.g., Klingberg et al., 2002a; Von Bastian \& Oberauer, 2013). However, it is well known that WM training can lead to increases in performance in non-practiced WM tasks (see reviews by Klingberg, 2010; Morrison \& Chein, 2011), but decreases have not yet been reported.

These results were not as expected, bearing in mind our prediction that transfer of WM and RI training combined with EC would lead to an additive effect of strengthening the explicit and implicit determinants of three safer-sex behaviors after five gaming sessions. This prediction, while explorative in nature, was apparently too optimistic. It is known that for WM training to be effective in terms of all its subcomponents (storage, processing, relational integration, and supervision; see von Bastian, Langer, Jäncke, \& Oberauer, 2013), it takes at least four weeks of extensive training of one specific functional category alone. In light of this, it is even more surprising that adding WM training seems to influence the strength and direction of several RAA determinants. However, targeting three behaviors with three different interventions in one game is perhaps simply too much for the short amount of gaming sessions.

\section{Limitations}

As this study was conducted online, our ability to ensure the careful and conscientious implementation of participants was limited. Therefore, we cannot rule out the possibility 
that some participants may not have followed the rather complex instructions (e.g., about playing time, taking a break, etc.), or may have been distracted by parallel activities. Second, it was not possible to observe actual behavior. We were only able to measure participants' predictions of their future behavior. In fact, we only used the RAA variables that directly predict behavioral intention, without measuring actual behavior. Third, the evaluative conditioning component was acted out using the avatar. This raises the question of whether the effectiveness of EC was influenced by the fact that it was the avatar and not the participant steering the avatar that the EC component was applied to. Furthermore, the stimuli normally used in EC paradigms to pair dislike with a neutral stimulus are pictures that are disgusting in nature. However, we did not use this method of conditioning and instead used the combination of a red speech bubble with an annoying horn sound and a loss of gaming points to evoke (pair) dislike with a unsafe-sex decision. It is also worth noting that the stimulus to be conditioned was very likely not neutral to start with, as it implied a decision to have no sex, unsafe-sex, or safe-sex. This may have further comprised the reliability of our EC condition. Fourth, we did not include a condition with active WM training only, and mock EC and RI training versions. Without measuring whether the WM training alone could influence explicit and implicit post-intervention measurements, we cannot conclude that it was the WM training that influenced the effects observed in the conditions where all components were active. Also, this study limited the number of WM training sessions to five, which might have been insufficient to induce an effect. Lastly, studies have shown that selective inhibition training seems not to have an effect on general inhibition abilities, but instead on automatic associations (e.g., Houben, Havermans, Nederkoorn, \& Jansen, 2012; Houben, Nederkoorn, Wiers, \& Jansen, 2011). Even if effects on general inhibition training were expected, the number of sessions might not have been enough to induce such effects.

\section{Conclusion}

We developed a serious game with the aim of strengthening implicit and explicit determinants of three safer-sex behaviors. The game delivered mixed results. Only when all three interventions (evaluative conditioning, working memory training, and selective response inhibition) were active were some explicit determinants strengthened while others, in particular intentions, were weakened after three or five gaming sessions. The reasons for this lack in transfer of cognitive training to behavioral determination remain unclear. We suggest, however, that five gaming sessions were not enough to achieve a robust attitude change and that targeting three behaviors in one game might have been too much. It seems that, also in a virtual environment, it could be a case of less is more. 


\section{Chapter Seven}

General Discussion 

The overall aim of this doctoral thesis is to report on a novel, serious game approach to increase safer-sex behavior in young people. As most sex education programs and studies on sexual risk behavior are focused on the use of barrier protection, such as condoms, we broadened this focus by adding the following two behaviors: asking about a new sex partner's sexual health and the refraining from risky sexual practices, such as penetrative anal sex, with a new sex partner. These three sexual health protecting behaviors have been identified to reduce the risk of acquiring STIs (Varghese et al., 2002). The serious game that was designed and programmed for this project aimed at increasing the implementation of these three protective behaviors in young people, aged between 16 and 24 years.

We found that young people's intentions to engage in these three protective safersex behaviors with a new sex partner were mainly explained by concepts from Fishbein and Ajzen's (2010) Reasoned Action Approach (see chapter 1). For example, perceived behavioral control predicted nearly $80 \%$ of condom use intentions. But besides RAA variables there were distal variables that also added significant predictive power to the intention to use condoms, such as the number of instances of unprotected intercourse during the last year. However, for the intention - with a new sex partner - to use condoms, to ask about his or her current sexual health status, and to refrain from risky sex practices, perceived behavioral control in conjunction with social norms were strong correlates for all three behaviors. Qualitative data (see chapter 2 ) showed that condom use was often perceived as a pleasure-reducing "necessary evil" but still identified as a behavior which protects against the contraction of STIs. This protective quality was also accorded to both engaging in less risky sex acts and asking about a partner's sexual health status (at least during first-time sex with a new sex partner). However, these protective measures were least popular among young people. The idea of engaging in less risky sex acts was repeatedly associated with boredom and rejected on the grounds of reduced sexual pleasure while asking a new sex partner about her or his sexual health status was often perceived as embarrassing and unpleasant, leading to widespread rejection mostly within subgroups reporting low intentions to perform all three protective behaviors. The rejection of asking a new sex partner about her or his current sexual health status was not observed when participants were sexually aroused, reflected in significantly more positive implicit and explicit attitudes towards this behavior as compared to being in a non-aroused state (see chapter 4). This suggests that behavioral disinhibition due to sexual arousal is not per se a threat to safer-sex behavior but can also support protective behavior that is perceived as embarrassing in a non-aroused state. Further, embarrassment can also hamper the translation of condom use intentions into behavior (see chapter 5). In fact, a high level of intention alone may be insufficient to predict condom use behavior if its implementation is associated with feelings of embarrassment.

To test if a combination of gamified behavior-change techniques - namely evaluative conditioning, selective response inhibition training, and working memory training would significantly increase the intentions to perform the three protective behaviors of 
interest, we developed a serious game. As the target population consisted of young people aged between 16 and 24 years, we invited females and males of that age group to share their preferences regarding content and design during focus group interviews. Participants preferred a real-world design with highly customizable avatars and a game that contained information modules to explain various safer-sex approaches and the use of different barrier protection methods. Participants emphasized the importance of including an alcohol intoxicated avatar to increase awareness about the loss of control that can occur while in an intoxicated state (see chapter 3).

The final game, called Heroes of the Night, aimed at strengthening implicit and explicit determinants of the three safer-sex behaviors of interest, however, delivered mixed results (see chapter 6). Only when the evaluative conditioning, working memory training, and selective response inhibition components were active during gaming, some explicit determinants were strengthened while others, especially intentions, were weakened after three or five gaming sessions. The reasons for this lack in transfer of cognitive training to behavioral determination remain unclear. We suggest, however, that five gaming sessions were not enough to achieve a robust attitude change and that targeting three behaviors in one game might have been too much. It seems that, also in a virtual environment, it could be a case of less is more.

\section{Determinants of behavior}

The dual-process approach (Strack \& Deutsch, 2004) that accounts for reasoning, judgment, and social cognition (Evans, 2008), describes a joint function of reflective and impulsive processes that interact following different operating systems. This joint function is based on the distinction between deliberate - explicit - attitudes and automatic - implicit - attitudes (for a review, see Gawronski \& Bodenhausen, 2006). Also, impulsive and reflective precursors of a specific behavior and situational or dispositional conditions were suggested to enable a better prediction of self-control outcomes compared to being analyzed on their own (Hofmann et al., 2009). Furthermore, understanding which variables predict a specific behavior in a well-defined target group is essential for the development of successful behavior-change interventions (for examples, see Bartholomew Eldredge, Markham, Ruiter, Fernandez, \& Parcel, 2016). Using the Reasoned Action Approach (Fishbein \& Ajzen, 2010) - which suggests that behavioral decisions are made based on slow and reasoned considerations derived from available information -, we identified perceived behavioral control (and to a lesser extend social norms) as a main predictor of the intention to perform all three safer-sex behaviors of interest (see chapter 1). However, due to the lack of attitude measurements, this result must be taken with precaution. Besides RAA variables, the number of instances of unprotected sexual intercourse during the last twelve months added predictive power (although less than RAA variables) and was identified as a moderating variable of perceived behavioral control 
related to intention to use condoms. The role of distal variables in increasing predictive power of behavioral models has been reported previously, for example, to reduce toxic chemical exposure among new and expectant mothers (Mello \& Hovick, 2016), or to explain the relationships between risk perceptions and vaccination behavior (Brewer et al., 2007). However, other distant variables, such as the level of risk-seeking behavior (concerning the intention to refrain from risky sex acts), or parenting style and unprotected sexual activity (concerning the intention to ask for the current health status) did not show unique contributions in multivariate analyses with intentions but showed significant univariate associations with those intentions. This lack of prediction power might result from the novelty of these target behaviors for young people or simply because these are more distal variables and thus mediated by more proximal ones, such as RAA variables (see chapter 2).

The reported predictive power of distal and RAA variables did not change significantly when participants were in a state of sexual arousal (see chapter 4). In such a state, only positive and negative life expectations were amplified compared to a non-aroused state. However, sexual arousal increased positive explicit and implicit attitudes towards asking a new sex partner about her or his current sexual health status. As engaging in uncommon and unprotected sexual behavior is usually higher in acute states of sexual arousal (e.g., Imhoff \& Schmidt, 2014; Skakoon-Sparling, Cramer, \& Shuper, 2015), this arousal might also facilitate protective health behavior, such as asking about possible STIs while profiting from a greater disinhibition than in a non-aroused state. In contrast, explicit, but not implicit, attitudes towards condom use were significantly more positive in a non-aroused state as compared to a sexually aroused state where condoms are often perceived as necessary but unpleasant, with the latter aspect likely reinforced during sexual arousal due to an increase in salience of mating goals (Hill \& Durante, 2011b). However, our findings show that all three safer-sex behaviors were believed to protect against STIs but the intention to implement these behaviors during the next first-time sexual encounter with a new sex partner showed a great variation (see chapter 2 ).

Even if not sexually aroused and with high intentions to use condoms with a new sex partner, a commonly cited reason for refraining from using condoms (and engaging in risky sex acts) was reduced sexual pleasure while not ignoring a possible health threat due to unprotected sexual intercourse. Reduced sexual pleasure as a barrier to condom use has been reported consistently in previous research (e.g., Randolph, Pinkerton, Bogart, Cecil, \& Abramson, 2007). However, studies also found that condom use did not reduce sexual pleasure (e.g., Sanders et al., 2010), especially not for young people who are in a relationship (Milhausen et al., 2018). In fact, sexual pleasure has been identified as a decisive factor in using condoms (Higgins \& Hirsch, 2008; Philpott, Knerr, \& Boydell, 2006), showing the complexity of different condom use determinants (Helweg-Larsen \& Collins, 1994). Also, in our sample, participants who were low in intention to implement all three behaviors did not acknowledge the risk of contracting an STI when not using condoms. Such a risk denial has previously been reported (e.g., Chanakira, O'Cathain, 
Goyder, \& Freeman, 2014) and needs to be addressed in appropriate sex education programs, such as contraceptive and safer-sex counseling in communities or educational institutions (Tiblom Ehrsson et al., 2016).

Peers of participants high in intention to adopt all three safer-sex behaviors were mostly believed to be approving of condom use, while the majority of participants with a low intention to adopt all three safer-sex behaviors indicated that their peers would disapprove of condom use. This relation between peer influence and sexual values has been described before (Wetherill, Neal, \& Fromme, 2010) and was corroborated in our study. Further, parents and family members were identified as important normative referents who would approve of all three protective behaviors and a normative power of health care professionals was also identified, especially for females high in intention to perform all three safer-sex behaviors. This is in line with previous research, linking parenting style (especially parent-adolescent communication) with safer-sex behavior (e.g., Widman, Choukas-Bradley, Noar, Nesi, \& Garrett, 2016; Widman, Noar, Choukas-Bradley, \& Francis, 2014). Also, a new sex partner who supports all three safer-sex behaviors, as well as a new sex partner who is perceived as trustworthy, were both seen as facilitating factors. Such an influence of normative behaviors has been linked previously to safer-sex behavior. Hyde, Drennan, Howlett, and Brady (2008) reported that safer-sex decisions were not merely established in individual discussions, but rather were produced discursively in a wider social setting that includes young people's broader perspectives on sexuality.

Behavioral intention is seen as a strong predictor of actual behavior (Fishbein \& Ajzen, 2010). However, simply having good intentions to use condoms may not result in actual condom use (which means that the intention may not be translated into condom use behavior) if condoms and condom use are associated with potentially embarrassing situations (see chapter 5). Such an association of embarrassment with condom use negotiation could represent a barrier to consistent condom use with a new sex partner among young adult populations. Even if participants with inconsistent past condom use behavior explicitly reported their intention to use condoms during first time sex with a new partner, it was likely the embarrassing information related to condoms that may have caused the more efficient disengagement of attention from even slightly embarrassing condomrelated pictures, compared to intention-behavior consistent participants. This finding is in line with studies showing that behavioral intentions appear to account for just $30 \%$ of the variance in actual behavior (Armitage \& Conner, 2001; Sheeran, 2002). This inconsistency between intention and behavior is referred to as "the intention-behavior gap" (Sheeran, 2002), pointing at the need for a better understanding of the mechanisms that determine the strength of the link between intention and behavior. 


\section{Gamification of behavior-change interventions}

Digital gaming interventions, commonly known as serious or educational games, have emerged as a novel way of delivering behavior-change interventions to a population of young people (Charsky, 2010; Enah et al., 2013) and shown to facilitate positive outcomes in participants (Wouters et al., 2013), even if they fail to induce long-term effects yet (DeSmet et al., 2014b). They may be uniquely suitable to present evidence-based training paradigms in an attractive and motivating training environment to improve behavioral control in young people (e.g., Boendermaker, Veltkamp, \& Peeters, 2017), but these games are still novel, and the literature about their effectiveness is rather fragmented and suffers from inconsistent referencing patterns between different disciplines (CastroSánchez, Kyratsis, Iwami, Rawson, \& Holmes, 201 ॠeyertheless, as tailoring behaviorchange interventions to the needs of a specific target group has now become an evidence-based approach (e.g., Bartholomew Eldredge et al., 2016), the tailoring of game content in educational games for sexual health promotion has also been shown to be effective (Noar et al., 2009; Portnoy et al., 2008). However, as DeSmet et al. (2016) pointed out, the effectiveness of tailoring might also reach a limit as a participatory design approach was less effective in changing health behavior compared to an approach that integrated the target group as testers in the game. Nevertheless, tailoring the game design to players' needs (especially their personality type) may still improve the effectiveness in promoting intentions to change behavior, as recently demonstrated by Orji, Mandryk, and Vassileva (2017). In chapter 3, we showed that design preferences of young people toward an educational game promoting safer sex did not differ depending on their cultural background. Young people preferred a design that reflects a real-world scenario (not a fantasy world) and includes realistic settings and daily activities. One important way of creating real-world scenarios is to increase self-identification with the virtual avatar that is supposed to depict the player (although there are limits to its effectiveness, see DeSmet et al., 2016), preferably by using a first-person visual perspective. This is also reflected in the importance participants placed on being able to customize their avatar. Content-wise, young people's preferences did also not differ depending on their cultural background. The only gender variation observed was related to how the topic of having sexual intercourse should be approached by the game. The only gender-related difference we found was that males preferred a more direct approach when it comes to introducing sexual intercourse in the game, whereas female participants favored a slightly softer approach that would be embedded in a narrative but nevertheless needed to remain realistic. 


\section{The effectiveness of the game}

Some digital-based interventions for health have proven to be effective, as shown, for example, by the "Re-mission" game - a digital health intervention that improved adherence to medical treatments and understanding of cancer in young adults with cancer (Kato, Cole, Bradlyn, \& Pollock, 2008) -, or the game "PR:EPARe" to be used in classroom settings for sex education (Arnab et al., 2013). However, as shown in meta-analyses by DeSmet et al. (2014) and DeSmet, Shegog, Van Ryckeghem, Crombez, and De Bourdeaudhuij (2015), serious games can positively affect determinants of a healthy lifestyle but often fail at changing behavior. This lack of transition from determinants to actual behavior after a serious game intervention has also been observed in other domains, for example, energy saving in an office environment (Orland et al., 2014). However, serious games were reported to be effective as educational tools (for meta-analyses see Girard, Ecalle, \& Magnan, 2013; Wouters et al., 2013), for example, in promoting the motivation to learn (Erhel \& Jamet, 2013). However, the transfer of factual knowledge, measured directly after the learning process, was shown to be as effective as textbook learning (Wardaszko \& Podgórski, 2017). Nevertheless, learning executive function skills by playing video games was reported to effectively transfer the cognitive skill trained in a game context to performance on the same skill in a non-game context (Parong et al., 2017).

Our novel serious game combined known techniques applied in behavior-change evaluative conditioning, selective response inhibition training, and working memory training - that have shown to be effective in non-gamified settings (e.g., Chiu et al., 2018), even if studies also failed to show their effectiveness (e.g., Gibson, 2008; Guye \& von Bastian, 2017; Verbeken, Braet, Naets, Houben, \& Boendermaker, 2018). When testing our serious game, we found mixed results depending on the combination of techniques and the number of gaming sessions. The condition with all three components in active mode revealed several significant differences between pre- and post-gaming measurements in explicit, but not in implicit behavioral determinants. However, most importantly, the intention to ask a new partner about his or her current sexual health status was weaker after three gaming sessions. Also, the intention to refrain from less risky sex practices was weaker after three and even weaker after five gaming sessions. Similar results have been observed previously in studies testing educational interventions on vaccination attitudes of young people. In a study testing the effectiveness of messages to reduce vaccine misperceptions, some communication strategies about vaccines actually increased misperceptions or reduced vaccination intention (Nyhan, Reifler, Richey, \& Freed, 2014). In another study, a 6-months follow-up of young people who received a digital or a presentation intervention aimed at strengthening attitudes toward vaccination showed that both groups shifted to a less enthusiastic attitude towards vaccination, as compared to a control group that showed stronger positive attitudes (Carolan et al., 2018). 
The factors contributing to the reduction of intention in our game are still unclear but targeting three behaviors with three different intervention in one game is maybe simply too much for the short amount of gaming sessions.

\section{Limitations}

The studies of this dissertation have some limitations. First, most studies presented only a limited sample of young people. As people in this age group (16-24 years) show a variety of (also gender-related) preferences in different domains of life (e.g., Gore et al., 2011; United Nations, 2015), our sample sizes were not representative of this entire population. Especially not if taking cultural differences into account. Therefore, care should be taken in applying our results to various cultural or ethnic subgroups. Also, our rather small sample size concerning qualitative assessments makes it difficult to determine whether data saturation was finally reached. This also holds true for males versus females as our limited sample of participants could lack data present in a larger sample.

Second, even if we included distal variables in some of our studies, we mainly based our studies on the predictive validity of a social cognition approach to behavior. Precisely, we used the time-tested Reasoned Action Approach (Fishbein \& Ajzen, 2010) which outlines the factors that determine an individual's behavioral decision. However, this is not the only theoretical approach to predict behavior. Other models, for instance the Prototype-willingness model (e.g., Gibbons, Gerrard, Blanton, \& Russell, 1998) that takes less reasoned pathways into account or the health belief model (for a meta-analysis, see Carpenter, 2010) that includes individual perceptions of threat and evaluation of behaviors to counter that threat, represent other approaches to understand young people's sexual risk behavior (for an overview, see Conner \& Norman, 2015). We cannot exclude that another model or even a combination of different models would have been better adapted to understand and predict young people's sexual risk behavior.

Third, some studies were conducted online, including the testing of the serious game. This, of course, reduced our ability to control for a careful and conscientious implementation by participants. Therefore, we cannot exclude that some participants may not have followed all instructions or may have been distracted by parallel activities.

Fourth, it was not possible to observe actual behavior so that we were only able to measure participants' predictions of their behavior. In fact, we only used the RAA variables that directly predict behavioral intention, without measuring the actual behaviors of interest. Such measurements - even if relying mostly on self-reports with sometimes limited validity (for meta-analyses relating to different behaviors, see, e.g., Berg, Peterson, Frazier, \& Crow, 2011; Helmerhorst, Brage, Warren, Besson, \& Ekelund, 2012; Johns \& Miraglia, 2015; Napper, Fisher, Reynolds, \& Johnson, 2010) - could have provided valuable information about the effects of additional distal predictors of behavior on the actual behavior and not only at behavioral intentions. 


\section{Implications for future research}

Due to the variety of preferences in different domains of life, studies conducted with young people (aged between 16-24 years) would greatly profit from a large sample size that is representative of this particular population, especially if cultural differences and gender roles are examined.

Even if the Reasoned Action Approach (Fishbein \& Ajzen, 2010) is a time-tested approach to predict behavioral decisions, it is not the only model that can be used to predict behavior. Studies focusing on such complex behavior as safer-sex could greatly profit from testing the predictive validity of different models on their outcome variables.

Conducting studies online can be advantageous if one needs to reach a large population that cannot easily be invited into a laboratory setting. However, to remain in control of a careful and conscientious implementation by participants, it might be advisable to organize rooms - for example in community centers or schools - where participants can take an online study in a controlled - but not surveilled - setting.

Intentions have been identified as important predictors of behavior (e.g., Fishbein \& Ajzen, 2010). However, intentions are not always translated into behavior - an inconsistency referred to as "the intention-behavior gap" (Sheeran, 2002). The only approach to know if intentions are translated into behavior is to measure the behavior of interest and not only to measure the likelihood of which participants intend to perform this behavior. Therefore, measuring behavior at different points in time after an intervention, for example, 3-months and 6-months post intervention, might be a more reliably way of assessing the success of a behavior-change intervention than measuring only behavioral intentions.

\section{Suggestions for future research}

Our results indicate the importance of some distal and RAA variables, especially perceived behavioral control related to using condoms. Increasing our understanding of whether and, if so, how RAA and distal variables interact could help to understand better and predict young people's sexual risk behavior. For example, understanding how past condom nonuse as a distal variable moderates the RAA variable perceived behavioral control, and in this way regulates future action, could help in the design of interventions that simulate successful past condom use behavior among nonusers in order to eventually increase perceived behavioral control related to condom use.

We conducted qualitative interviews to understand what young people from different cultural backgrounds expect from a serious game that aims at promoting safer-sex behavior. We were able to conduct this study with only a limited sample of young people from one of just two different cultural backgrounds (young people raised in Germany by their Turkish families and young people raised by their German families). However, 
different results might be found in relation to young people with yet a different cultural background. Future studies could include people from more cultural backgrounds to have a better understanding if safer-sex interventions need to be tailored towards specific cultural needs (e.g., specific does and don'ts of different cultures) of young people.

Further, as sexual decision making is very likely to happen during a state of sexual arousal, it would be interesting to test gamified behavior-change interventions not only during a non-aroused state but also during a sexually aroused state. Also, the use of physiological methods to measure participants' level of sexual arousal would be preferable instead of relying on self-reported data that have shown to weakly (Chivers et al., 2010) or even negatively (Grauvogl et al., 2015) correlate with genital measures of sexual arousal, such as vaginal pulse amplitudes.

Lastly, it was not possible to observe actual behavior so that we were only able to measure participants' predictions of their behavior (Bouffard, 2002; Loewenstein et al., 1997). However, intentions are not necessarily translated into behavior, an observation known as the intention-behavior gap (Sheeran, 2002). Assessing safer-sex behavior several months after an intervention would help to identify which variables predict actual behavior and what might cause a non-translation of intentions into behavior.

\section{Conclusion}

We designed a serious game to increase the implementation of three protective safersex behaviors in young people (aged between 16 and 24 years) with a new sex partner, (1) using condoms, (2) asking about her or his partner's current sexual health status, and (3) refraining from risky sex practices. We found that young people's intentions to engage in these three protective safer-sex behaviors with a new sex partner were mainly predicted by Fishbein and Ajzen's (2010) Reasoned Action Approach. Condom use was often perceived as a pleasure-reducing "necessary evil" but still identified as a behavior which protects against the contraction of STIs. However, even if condom use intentions were high, embarrassment could hamper the translation of these intentions into behavior. The protective quality of condom use was also accorded to both engaging in less risky sex acts and asking about a partner's sexual health status (at least during first-time sex with a new sex partner), but these protective measures were least popular among young people. However, the rejection of asking a new sex partner about her or his current sexual health status was not observed when participants were sexually aroused. Being sexually aroused they had significantly more positive implicit and explicit attitudes towards this behavior as compared to being in a non-aroused state, suggesting that behavioral disinhibition due to sexual arousal is not per se a threat to safer-sex behavior but can also support protective behavior that is perceived as embarrassing in a non-aroused state.

Related to the serious game, participants preferred a real-world design with highly customizable avatars and a game that contained information modules to explain various 
safer-sex approaches and the use of different barrier protection methods. Participants also emphasized the importance of including an alcohol intoxicated avatar to increase awareness about the loss of control that can occur while in an intoxicated state. However, the testing of the final game - aimed at strengthening implicit and explicit determinants of the three safer-sex behaviors of interest by using evaluative conditioning, working memory training, and behavioral inhibition training - delivered mixed results. Only when all components were active during gaming, some explicit determinants were strengthened while others, especially intentions, were weakened after three or five gaming sessions. We suggest that five gaming sessions were not enough to achieve a robust attitude change and that targeting three behaviors in one game might have been too much. It seems that, also in a virtual environment, it could be a case of less is more. 
References 
Aiken, L. S., \& West, S. G. (1991). Multiple regression: Testin and interpreting interactions. London: Sage.

Ajzen, I. (1985). From intentions to actions: A theory of planned behavior. In J. Kuhl \& J. Beckmann (Eds.), Action control: From cognition to behavior (pp. 11-39). Heidelberg: Springer.

Ajzen, I. (1991). The theory of planned behavior. Organizational Behavior and Human Decision Processes, 50(2), 179-211.

Ajzen, I., \& Driver, B. L. (1991). Prediction of leisure participation from behavioral, normative, and control beliefs: An application of the theory of planned behavior. Leisure Sciences, 13(3), 185-204. https://doi.org/10.1080/01490409109513137

Albarracín, D., Gillette, J. C., Earl, A. N., Glasman, L. R., Durantini, M. R., \& Ho, M.-H. (2005). A Test of Major Assumptions About Behavior Change: A Comprehensive Look at the Effects of Passive and Active HIVPrevention Interventions Since the Beginning of the Epidemic. Psychological Bulletin, 131(6), 856-897. https://doi.org/10.1037/0033-2909.131.6.856

Albarracín, D., Johnson, B. T., Fishbein, M., \& Muellerleile, P. A. ( $\left.\begin{array}{llll}2 & 0 & 0 & 1\end{array}\right)$. Theories of reasoned action andmed behavior as models of condom use: a meta-analysis. Psychological Bulletin, 127(1), 142-161.

Albert, D., Chein, J., \& Steinberg, L. (2013). The Teenage Brain: Peer Influences on Adolescent Decision Making. Current Directions in Psychological Science, 22(2), 114-120. https://doi.org/10.1177/ 0963721412471347

Albert, D., \& Steinberg, L. (2011). Judgment and decision making in adolescence. Journal of Research on Adolescence, 21(1), 211-224. https://doi.org/10.1111/j.1532-7795.2010.00724.x

All, A., Nuñez Castellar, P. E., \& Looy, J. Van. (2014). Measuring Effectiveness in Digital Game-Based Learning: A Methodological Review. International Journal of Serious Games, 1(2), 3-21. https://doi.org/10.17083/ ijsg.v1i2.18

Allom, V., Mullan, B., \& Hagger, M. (2016). Does inhibitory control training improve health behaviour? A metaanalysis. Health Psychology Review, 10(2), 168-186. https://doi.org/10.1080/17437199.2015.1051078

Ames, S. L., Grenard, J. L., \& Stacy, A. W. (2013). Dual process interaction model of HIV-risk behaviors among drug offenders. AIDS and Behavior, 17(3), 914-925.

Amir, N., Beard, C., Taylor, C. T., Klumpp, H., Elias, J., \& Chen, X. (2010). Attention Training in Individuals with Generalized Social Phobia: A Randomized Controlled Trial. Burns, 77(5), 961-973. https://doi.org/ 10.1037/a0016685.Attention

Amir, N., Elias, J., Klumpp, H., \& Przeworski, A. (2003). Attentional bias to threat in social phobia: facilitated processing of threat or difficulty disengaging attention from threat? Behaviour Research and Therapy, 41, 1325-1335. https://doi.org/10.1016/S0005-7967(03)00039-1

Anderson, S.-J., Cherutich, P., Kilonzo, N., Cremin, I., Fecht, D., Kimanga, D., ... Hallett, T. B. (2014). Maximising the effect of combination HIV prevention through prioritisation of the people and places in greatest need: a modelling study. The Lancet, 384(9939), 249-256. https://doi.org/10.1016/S0140-6736(14) 61053-9

Andrés, P. (2003). Frontal Cortex as the Central Executive of Working Memory: Time to Revise Our View. Cortex. https://doi.org/10.1016/S0010-9452(08)70868-2

Andrews, J. A., Tildesley, E., Hops, H., \& Li, F. (2002). The influence of peers on young adult substance use. Hea Ith Psych olog y: Officia I Jou rn a I of the Division of Hea Ith Psych olog y, America $n$ Psych olog Association, 21(4), 349-357.

Annetta, L. A. (2010). The "I's" have it: A framework for serious educational game design. Review of General Psychology, 14(2), 105-112.

Ariely, D., \& Loewenstein, G. (2006). The heat of the moment: The effect of sexual arousal on sexual decision making. Journal of Behavioral Decision Making, 19(2), 87-98. https://doi.org/10.1002/bdm.501

Armitage, C. J., \& Conner, M. (2001). Efficacy of the Theory of Planned Behaviour: A meta-analytic review. British Journal of Social Psychology, 40, 471-499.

Arnab, S., Brown, K., Clarke, S., Dunwell, I., Lim, T., Suttie, N., ... de Freitas, S. (2013). The development approach of a pedagogically-driven serious game to support Relationship and Sex Education (RSE) within a classroom setting. Computers \& Education, 69, 15-30. https://doi.org/10.1016/j.compedu.2013.06.013

Arnett, J. (1992). Reckless behavior in adolescence: A developmental perspective. Developmental Review, 12(4), $339-373$. 
Aron, A. R. (2011). From reactive to proactive and selective control: developing a richer model for stopping inappropiate responses. Biological Psychiatry. https://doi.org/10.1016/j.biopsych.2010.07.024.From

Ashcraft, C. (2003). Adolescent Ambiguities in American Pie: Popular Culture as a Resource for Sex Education. Youth \& Society, 35(1), 37-70.

Avery, L., \& Lazdane, G. (2010). What do we know about sexual and reproductive health of adolescents in Europe? The Eu rop ea $n$ Jou $r n$ a I of Con tra cep tion \& Rep rod u ctive Hea Ith Ca re: Th e Officia I Jou rn a European Society of Contraception, 15 Supp/ 2(December), S54-S66. https://doi.org/10.3109/13625187. 2010.533007

Baayen, R. H., \& Milin, P. (2010). Analyzing Reaction Times. International Journal of Psychological Research, 3(2), $12-28$

Baddeley, A. (1992). Working memory. Science, 255(5044), 556-559. https://doi.org/10.1126/science. 1736359

Baddeley, A. (1998). The central executive: A concept and some misconceptions. Journal of the International Neuropsychological Society, 4(05), 523-526.

Baddeley, A. (2012). Working memory: theories, models, and controversies. Annual Review of Psychology, 63, $1-29$.

Baddeley, A., \& Della Sala, S. (1996). Working memory and executive control. Philosophical Transactions of the Royal Society of London. Series B, Biological Sciences, 351(1346), 1397-403; discussion 1403-4. https://doi.org/10.1098/rstb.1996.0123

Baele, J., Dusseldorp, E., \& Maes, S. (2001). Condom use self-efficacy: Effect on intended and actual condom use in adolescents. Journal of Adolescent Health, 28(5), 421-431.

Ballonoff Suleiman, A., \& Brindis, C. D. (2014). Adolescent school-based sex education: Using developmental neuroscience to guide new directions for policy and practice. Sexuality Research and Social Policy, 11(2), 137-152. https://doi.org/10.1007/s13178-014-0147-8

Ballonoff Suleiman, A., Johnson, M., Shirtcliff, E. A., \& Galván, A. (2 01015 ). ScłBaokd Sex Education and Neuroscience: What We Know About Sex, Romance, Marriage, and Adolescent Brain Development. Journal of School Health, 85(8), 567-574. https://doi.org/10.1111/josh.12285

Bandura, A. (1986). Social foundations of thought and action. New York: Prentice-Hall.

Bantin, T., Stevens, S., Gerlach, A. L., \& Hermann, C. (2016). What does the facial dot-probe task tell us about attentional processes in social anxiety? A systematic review. Journal of Behavior Therapy and Experimental Psychiatry, 50, 40-51. https://doi.org/10.1016/j.jbtep.2015.04.009

Bar-Anan, Y., De Houwer, J., \& Nosek, B. A. (2012). Evaluative conditioning: Recent developments and future directions. Learning and Motivation, 43(3), 79-88. https://doi.org/10.1016/j.Imot.2012.06.004

Bari, A., \& Robbins, T. W. (2013). Inhibition and impulsivity: Behavioral and neural basis of response control. Progress in Neurobiology. https://doi.org/10.1016/j.pneurobio.2013.06.005

Bartholomew Eldredge, L., Markham, C., Ruiter, R. A. C., Fernandez, M., \& Parcel, G. (2016). Planning health $p$ romotion $p$ rog ra $m s$ : An in terven tion ma $p$ p. Nhevg JerspypjorsceyeBass.

Bauman, L. J., \& Berman, R. (2005). Adolescent relationships and condom use: Trust, love and commitment. AIDS and Behavior, 9(2), 211-222.

Bauman, L. J., Karasz, A., \& Hamilton, A. (2007). Understanding Failure of Condom Use Intention Among Adolescents: Completing an Intensive Preventive Intervention. Journal of Adolescent Research, 22(3), 248274.

Baumrind, D. (1978). Reciprocal rights and responsibilities in parent-child relations. Journal of Social Issues, 34(2), 179-196.

Baumrind, D. (1991). The Influence of Parenting Style on Adolescent Competence and Substance Use. The Journal of Early Adolescence, 11(1), 56-95.

Beard, C., Sawyer, A. T., \& Hofmann, S. G. (2012). Efficacy of Attention Bias Modification Using Threat and Appetitive Stimuli: A Meta-Analytic Review. Behavior Therapy, 43(4), 724-740. https://doi.org/10.1016/ j.beth.2012.01.002

Bell, K., Terzian, M. A., \& Moore, K. A. (2012). What works for female children and adolescents: Lessons from experimental evaluations of programs and interventions. 
Bellocchi, S., Henry, V., \& Baghdadli, A. (2017). Visual Attention Processes and Oculomotor Control in Autism Spectrum Disorder: A Brief Review and Future Directions. Journal of Cognitive Education and Psychology, 16(1), 77-93. https://doi.org/10.1891/1945-8959.16.1.77

Benson, J. E., Sabbagh, M. A., Carlson, S. M., \& Zelazo, P. D. (2013). Individual differences in executive functioning predict preschoolers' improvement from theory-of-mind training. Developmental Psychology. https://doi.org/10.1037/a0031056

Bentin, S., Mouchetant-Rostaing, Y., Giard, M. H., Echallier, J. F., \& Pernier, J. (1999). ERP manifestations of processing printed words at different psycholinguistic levels: time course and scalp distribution. Journal of Cognitive Neuroscience, 11(3), 235-260.

Berg, K. C., Peterson, C. B., Frazier, P., \& Crow, S. J. (2011). Convergence of scores on the interview and questionnaire versions of the Eating Disorder Examination: A meta-analytic review. Psychological Assessment, 23(3), 714-724. https://doi.org/10.1037/a0023246

Berndt, T. J. (1979). Developmental changes in conformity to peers and parents. Developmental Psychology, 15(6), 608-616.

Beyers, W., \& Goossens, L. (1999). Emotional autonomy, psychosocial adjustment and parenting: interactions, moderating and mediating effects. Journal of Adolescence, 22(6), 753-769.

Biglan, A., Metzler, C. W., Wirt, R., Ary, D., Noell, J., Ochs, L., ... Hood, D. (1990). Social and behavioral factors associated with high-risk sexual behavior among adolescents. Journal of Behavioral Medicine, 13(3), 245261. https://doi.org/10.1007/BF00846833

Birch, P. J., White, J. M., \& Fellows, K. (2017). The broad effectiveness of seventy-four field instances of abstinence-based programming. Sex Education, 17(1), 14-25. https://doi.org/10.1080/14681811. 2016.1176908

Birk, M. V., Atkins, C., Bowey, J. T., \& Mandryk, R. L. (2016). Fostering Intrinsic Motivation through Avatar Identification in Digital Games. In Proceedings of the 2016 CHI Conference on Human Factors in Computing Systems - CHI'16 (pp. 2982-2995). https://doi.org/10.1145/2858036.2858062

Birnbaum, G. E., Mikulincer, M., Szepsenwol, O., Shaver, P. R., \& Mizrahi, M. (2014). When sex goes wrong: a behavioral systems perspective on individual differences in sexual attitudes, motives, feelings, and behaviors. Journal of Personality and Social Psychology, 106(5), 822-842. https://doi.org/10.1037/ a0036021

Black, S. E., Devereux, P. J., \& Salvanes, K. G. (2013). Under Pressure? The Effect of Peers on Outcomes of Young Adults. Journal of Labor Economics, 31(1), 119-153.

Blais, A., \& Weber, E. U. (2006). A Domain-Specific Risk-Taking ( DOSPERT) scale for adult populations. Judgement and Decision Making, 1(1), 33-47. https://doi.org/10.1037/t13084-000

Blanton, H., Jaccard, J., \& Burrows, C. N. (2015). Implications of the Implicit Association Test D-Transformation for Psychological Assessment. Assessment. https://doi.org/10.1177/1073191114551382

Böcker, K. B. E., Baas, J. M. P., Leon Kenemans, J., \& Verbaten, M. N. (2004). Differences in startle modulation during instructed threat and selective attention. Biological Psychology, 67(3), 343-358.

Boekeloo, B., Boyle, M., Quinton, S., \& Rashaw, B. (2018). College Student Sexual Risks Associated with Firsttime Sex with Someone after Sexting Them. Health Behavior and Policy Review, 5(2), 3-11. https://doi.org/10.14485/HBPR.5.2.1

Boendermaker, W. J., Prins, P. J. M., \& Wiers, R. W. (2015). Cognitive Bias Modification for adolescents with substance use problems--Can serious games help? Journal of Behavior Therapy and Experimental Psychiatry, 49, 13-20. https://doi.org/10.1016/j.jbtep.2015.03.008

Boendermaker, W. J., Veltkamp, R. C., \& Peeters, M. (2017). Training Behavioral Control in Adolescents Using a Serious Game. Games for Health Journal, 6(6), 351-357. https://doi.org/10.1089/g4h.2017.0071

Boettcher, J., Berger, T., \& Renneberg, B. (2012). Internet-Based Attention Training for Social Anxiety: A Randomized Controlled Trial. Cognitive Therapy and Research, 36(5), 522-536. https://doi.org/10.1007/s10608-011-9374-y

Boettcher, J., Leek, L., Matson, L., Holmes, E. A., Browning, M., MacLeod, C., ... Carlbring, P. (2013). InternetBased Attention Bias Modification for Social Anxiety: A Randomised Controlled Comparison of Training 
towards Negative and Training Towards Positive Cues. PLOS ONE, 8(9), e71760. https://doi.org/ 10.1371/journal.pone.0071760

Bonevski, B., Randell, M., Paul, C., Chapman, K., Twyman, L., Bryant, J., ... Hughes, C. (2014). Reaching the hardto-reach: a systematic review of strategies for improving health and medical research with socially disadvantaged groups. BMC Medical Research Methodology, 14(1), 42. https://doi.org/10.1186/14712288-14-42

Borawski, E. A., Trapl, E. S., Lovegreen, L. D., Colabianchi, N., \& Block, T. (2005). Effectiveness of Abstinenceonly Intervention in Middle School Teens. American Journal of Health Behavior, 29(5), 423-434. https://doi.org/10.5993/AJHB.29.5.5

Both, S., Laan, E., \& Everaerd, W. (2011). Focusing "Hot" or Focusing "Cool": Attentional Mechanisms in Sexual Arousal in Men and Women. Journal of Sexual Medicine, 8(1), 167-179.

Bouffard, J. A. (2002). The influence of emotion on rational decision making in sexual aggression. Journal of Criminal Justice, 30(2), 121-134.

Bowerman, B., \& O'Connell, R. (1990). Linear Statistical Models: An Applied Approach. Belmont: Duxbury.

Bowling, J., Dodge, B., Bindra, N., Dave, B., Sharma, R., Sundarraman, V., ... Herbenick, D. (2018). Female condom acceptability in urban India: Examining the role of sexual pleasure. Journal of Health Psychology, 23(2), 218-228. https://doi.org/10.1177/1359105317745963

Bowring, A. L., Wright, C. J. C., Douglass, C., Gold, J., \& Lim, M. S. C. (2018). Features of successful sexual health promotion programs for young people: findings from a review of systematic reviews. Health Promotion Journal of Australia, 29(1), 46-57. https://doi.org/10.1002/hpja.3

Boyle, E. A., Connolly, T. M., Hainey, T., \& Boyle, J. M. (2012). Engagement in digital entertainment games: A systematic review. Computers in Human Behavior.

Braun, V., \& Clarke, V. (2006). Using thematic analysis in psychology. Qualitative Research in Psychology, 3(2), 77-101. https://doi.org/10.1191/1478088706qp063oa

Braver, T. S. (2012). The variable nature of cognitive control: A dual mechanisms framework. Trends in Cognitive Sciences, 16, 106-113. https://doi.org/10.1016/j.tics.2011.12.010

Braver, T. S., Gray, J. R., \& Burgess, G. C. (2007). Explaining the Many Varieties of Working Memory Variation: Dual Mechanisms of Cognitive Control. In A. Conway (Ed.), Variation in Working Memory (pp. 76-106). Oxford: Oxford University Press. https://doi.org/10.1093/acprof:oso/9780195168648.003.0004

Brewer, N. T., Chapman, G. B., Gibbons, F. X., Gerrard, M., McCaul, K. D., \& Weinstein, N. D. (2007). Metaanalysis of the relationship between risk perception and health behavior: The example of vaccination. Health Psychology, 26(2), 136-145. https://doi.org/10.1037/0278-6133.26.2.136

Bronfenbrenner, U. (1979). The ecology of human development. European Review (Vol. 13).

Brotonsc, C., Björkelund, C., Bulc, M., Ciurana, R., Godycki-Cwirko, M., Jurgova, E., ... Vuchak, J. (2005). Prevention and health promotion in clinical practice: The views of general practitioners in Europe. Preventive Medicine, 40(5), 595-601.

Brown, S., \& Locker, E. (2009). Defensive responses to an emotive anti-alcohol message. Psychology \& Health, 24(5), 517-528.

Brüll, P., Ruiter, R. A. C., Wiers, R. W., \& Kok, G. (2016). Gaming for Safer Sex: Young German and Turkish People Report No Specific Culture-Related Preferences Toward Educational Games Promoting Safer Sex. Games for Health Journal, 5(6). https://doi.org/10.1089/g4h.2016.0016

Buttram, M. E., Pagano, M. E., \& Kurtz, S. P. (2018). Frequency of Group Sex Participation and Risk for HIV/STI Among Young Adult Nightclub Scene Participants. International Journal of Sexual Health, 30(1), 12-19. https://doi.org/10.1080/19317611.2017.1385561

Carlbring, P., Apelstrand, M., Sehlin, H., Amir, N., Rousseau, A., Hofmann, S. G., \& Andersson, G. (2012). Internetdelivered attention bias modification training in individuals with social anxiety disorder--a double blind randomized controlled trial. BMC Psychiatry, 12, 66. https://doi.org/10.1186/1471-244X-12-66

Carolan, K., Verran, J., Crossley, M., Redfern, J., Whitton, N., \& Amos, M. (2018). Impact of educational interventions on adolescent attitudes and knowledge regarding vaccination: A pilot study. PLOS ONE, 13(1), e0190984. https://doi.org/10.1371/journal.pone.0190984 
Carpenter, C. J. (2010). A meta-analysis of the effectiveness of health belief model variables in predicting behavior. Health Communication, 25(8), 661-669. https://doi.org/10.1080/10410236.2010.521906

Carter, D. (2012). Comprehensive Sex Education for Teens Is More Effective than Abstinence. AJN The American Journal of Nursing, 112, 15.

Carver, C. S., \& White, T. L. (1994). Behavioral inhibition, behavioral activation, and affective responses to impending reward and punishment: The BIS/BAS Scales. Journal of Personality and Social Psychology, 67(2), 319-333.

Casey, B. J., Jones, R. M., \& Somerville, L. H. (2011). Braking and accelerating of the adolescent brain. Journal of Research on Adolescence, 21(1), 21-33. https://doi.org/10.1111/j.1532-7795.2010.00712.x

Casey, M. K., Timmermann, L., Allen, M., Krahn, S., \& Turkiewicz, K. L. (2009). Response and Self-Efficacy of Condom Use: A Meta-Analysis of this Important Element of AIDS Education and Prevention. Southern Communication Journal, 74(1), 57-78.

Cassotti, M., Agogué, M., Camarda, A., Houdé, O., \& Borst, G. (2016). Inhibitory Control as a Core Process of Creative Problem Solving and Idea Generation from Childhood to Adulthood. New Directions for Child and Adolescent Development. https://doi.org/10.1002/cad.20153

Cassotti, M., Aite, A., Osmont, A., Houde, O., \& Borst, G. (2014). What have we learned about the processes involved in the lowa Gambling Task from developmental studies? Frontiers in Psychology. https://doi.org/10.3389/fpsyg.2014.00915

Castro-Sánchez, E., Kyratsis, Y., Iwami, M., Rawson, T. M., \& Holmes, A. H. (2 $\begin{array}{llll}0 & 1 & 6\end{array}$ ). Serious electronic games as behavioural change interventions in healthcare-associated infections and infection prevention and control: a scoping review of the literature and future directions. Antimicrobial Resistance \& Infection Control, 5(1), 34. https://doi.org/10.1186/s13756-016-0137-0

Catalano, R. F., Fagan, A. a., Gavin, L. E., Greenberg, M. T., Irwin, C. E., Ross, D. a., \& Shek, D. T. L. (2012). Worldwide application of prevention science in adolescent health. The Lancet, 379(9826), 1653-1664. https://doi.org/10.1016/S0140-6736(12)60531-5

Cavanaugh, C., Mial, K., \& Tulloch, D. (2016). Assessing and Mapping the Availability of the Female Condom in the Philadelphia Metropolitan Area. AIDS and Behavior, 20(12), 2845-2849. https://doi.org/10.1007/ s10461-016-1317-x

Centers for Disease Control. (2011). Sexual identity, sex of sexual contacts, and health-risk behaviors among students in grades 9-12 - Youth risk behavior surveilance. Atlanta.

Centers for Disease Control. (2012). Sexually transmitted disease surveillance 2011. Atlanta.

Centers for Disease Control. (2015). Sexually transmitted disease surveillance 2014. Atlanta.

Centers for Disease Control and Prevention. (2014). Talking with Your Teens about Sex: Going Beyond "the Talk." Atlanta. Retrieved from https://www.cdc.gov/healthyyouth/protective/pdf/talking_teens.pdf

Centers for Disease Control and Prevention. (2017). Sexually Transmitted Disease Surveillance 2016. Atlanta. Retrieved from https://www.cdc.gov/std/stats16/CDC_2016_STDS_Report-for508WebSep21_2017_1644.pdf

Centers for Disease Control and Prevention. (2018). HIV Risk and Prevention. Retrieved June 1, 2018, from https://www.cdc.gov/hiv/risk/index.html

Chanakira, E., O'Cathain, A., Goyder, E. C., \& Freeman, J. V. (2014). Factors perceived to influence risky sexual behaviours among university students in the United Kingdom: a qualitative telephone interview study. BMC Public Health, 14(1), 1055. https://doi.org/10.1186/1471-2458-14-1055

Charsky, D. (2010). From Edutainment to Serious Games: A Change in the Use of Game Characteristics. Games and Culture, 5(2), 177-198.

Chein, J., Albert, D., O’Brien, L., Uckert, K., \& Steinberg, L. (2011). Peers increase adolescent risk taking by enhancing activity in the brain's reward circuitry. Developmental Science, 14(2), F1-F10. https://doi.org/10.1111/j.1467-7687.2010.01035.x

Chen, Z., Veling, H., Dijksterhuis, A., \& Holland, R. W. (2016). How does not responding to appetitive stimuli cause devaluation: Evaluative conditioning or response inhibition? Journal of Experimental Psychology: General, 145(12), 1687-1701. https://doi.org/10.1037/xge0000236 
Chikazoe, J., Jimura, K., Asari, T., Yamashita, K. I., Morimoto, H., Hirose, S., ... Konishi, S. (2009). Functional dissociation in right inferior frontal cortex during performance of go/no-go task. Cerebral Cortex. https://doi.org/10.1093/cercor/bhn065

Chin, H. B., Sipe, T. A., Elder, R., Mercer, S. L., Chattopadhyay, S. K., Jacob, V., ... Santelli, J. (2012). The Effectiveness of Group-Based Comprehensive Risk-Reduction and Abstinence Education Interventions to Prevent or Reduce the Risk of Adolescent Pregnancy, Human Immunodeficiency Virus, and Sexually Transmitted Infections. American Journal of Preventive Medicine, 42(3), 272-294. https://doi.org/ 10.1016/j.amepre.2011.11.006

Chiu, H.-L., Chan, P.-T., Kao, C.-C., Chu, H., Chang, P.-C., Hsiao, S.-T. S., ... Chou, K.-R. (2018). Effectiveness of executive function training on mental set shifting, working memory and inhibition in healthy older adults: A double-blind randomized controlled trials. Journal of Advanced Nursing, 74(5), 1099-1113. https://doi.org/10.1111/jan.13519

Chivers, M. L., Seto, M. C., Lalumiere, M. L., Laan, E., \& Grimbos, T. (2010). Agreement of self-reported and genital measures of sexual arousal in men and women: a meta-analysis. Archives of Sexual Behavior.

Christou, C., \& Michael, D. (2014). Aliens versus Humans: Do avatars make a difference in how we play the game? In 2014 6th International Conference on Games and Virtual Worlds for Serious Applications, VSGAMES 2014. https://doi.org/10.1109/VS-Games.2014.7012029

Cohen, J. (1988). Statistical power analysis for the behavioral sciences (2nd ed.). Lawrence Erlbaum Associates, Inc.

Cohen, J. (1992). A power primer. Psychological Bulletin, 112, 155-159.

Cohen, J., Cohen, P., West, S., \& Aiken, L. (2003). Applied multiple regression/ correlation analysis for the behavioral sciences (3rd ed.). Mahwah: Lawrence Erlbaum Associates, Inc.

Coleman, L., \& Testa, A. (2007). Preferences towards sex education and information from an ethnically diverse sample of young people. Sex Education, 7(3), 293-307.

Coley, R. L., Lombardi, C. M., Lynch, A. D., Mahalik, J. R., \& Sims, J. (2013). Sexual partner accumulation from adolescence through early adulthood: The role of family, peer, and school social norms. Journal of Adolescent Health, 53(1), 91-97. https://doi.org/10.1016/j.jadohealth.2013.01.005

Conley, T. D., Matsick, J. L., Moors, A. C., Ziegler, A., \& Rubin, J. D. (2015). Re-examining the effectiveness of monogamy as an STI-preventive strategy. Preventive Medicine, 78, 23-28. https://doi.org/10.1016/ j.ypmed.2015.06.006

Conner, M., \& Norman, P. (2015). Predicting and changing health behaviour: A social cognition approach. In M. Conner \& P. Norman (Eds.), Predicting and changing health behaviour. Research and practice with social cognition models (pp. 1-29). Berkshire: McGraw-Hill.

Connolly, T. M., Boyle, E. A., MacArthur, E., Hainey, T., \& Boyle, J. M. (2012). A systematic literature review of empirical evidence on computer games and serious games. Computers \& Education, 59(2), 661-686. https://doi.org/10.1016/j.compedu.2012.03.004

Conroy, M. A., \& Polich, J. (2007). Normative variation of P3a and P3b from a large sample: Gender, topography, and response time. Journal of Psychophysiology, 21(1), 22-32.

Consedine, N. S., Krivoshekova, Y. S., \& Harris, C. R. (2007). Bodily embarrassment and judgment concern as separable factors in the measurement of medical embarrassment: psychometric development and links to treatment-seeking outcomes. British Journal of Health Psychology, 12(Pt 3), 439-462.

Corneille, M. a., Tademy, R. H., Reid, M. C., Belgrave, F. Z., \& Nasim, A. (2008). Sexual safety and risk taking among African American men who have sex with women: A qualitative study. Psychology of Men \& Masculinity, 9(4), 207-220. https://doi.org/10.1037/a0012768

Cornelius, J. R., Clark, D. B., Reynolds, M., Kirisci, L., \& Tarter, R. (2007). Early age of first sexual intercourse and affiliation with deviant peers predict development of SUD: A prospective longitudinal study. Addictive Behaviors, 32(4), 850-854. https://doi.org/10.1016/j.addbeh.2006.06.027

Crockett, L. J., Raffaelli, M., \& Shen, Y. (2006). Psychology Linking Self-Regulation and Risk Proneness to Risky Sexual Behavior: Pathways through Peer Pressure and Early Substance Use Linking Self-Regulation and Risk Proneness to Risky Sexual Behavior : Pathways through Peer Pressure and Early Substanc, 16(4), 503525. 
Crone, E. a., \& Dahl, R. E. (2012). Understanding adolescence as a period of social-affective engagement and goal flexibility. Nature Reviews Neuroscience, 13(9), 636-650. https://doi.org/10.1038/nrn3313

Crosby, R. A., DiClemente, R. J., Salazar, L. F., Wingood, G. M., McDermott-Sales, J., Young, A. M., \& Rose, E. (2013). Predictors of Consistent Condom Use Among Young African American Women. AIDS and Behavior, 17(3), 865-871. https://doi.org/10.1007/s10461-011-9998-7

Crosby, R. A., Milhausen, R. R., Mark, K. P., Yarber, W. L., Sanders, S. A., \& Graham, C. A. (2013). Understanding problems with condom fit and feel: An important opportunity for improving clinic-based safer sex programs. Journal of Primary Prevention, 34(1-2), 109-115.

Crowe, N., \& Watts, M. (2012). 'When I click "ok" I become Sassy - I become a girl'. Young people and gender identity: subverting the 'body' in massively multi-player online role-playing games. International Journal of Adolescence and Youth, 19(2), 217-231.

Crowne, D., \& Marlowe, D. (1960). A new scale of social desirability independent of psychopathology. Journal Oj Consulting Psychology, 24(4), 349-354. https://doi.org/10.1037/h0047358

Cubbin, C., Brindis, C. D., Jain, S., Santelli, J., \& Braveman, P. (2010). Neighborhood poverty, aspirations and expectations, and initiation of sex. Journal of Adolescent Health, 47(4), 399-406. https://doi.org/ 10.1016/j.jadohealth.2010.02.010

Cugelman, B. (2013). Gamification: What It Is and Why It Matters to Digital Health Behavior Change Developers. JMIR Serious Games, 1(1), e3. https://doi.org/10.2196/games.3139

Curry, I., Scott, W. D., Bulotaite, L., \& Freng, S. (2017). The role of implicit associations and explicit expectancies related to alcohol use: a comparison of Lithuanian and US college samples. International Journal of Culture and Mental Health. https://doi.org/10.1080/17542863.2017.1296877

Cushman, N., Kantor, L. M., Schroeder, E., Eicher, L., \& Gambone, G. (2014). Sexuality education: findings and recommendations from an analysis of 10 United States programmes. Sex Education, 14(5), 481-496. https://doi.org/10.1080/14681811.2014.918538

Czopp, A., Monteith, M., Zimmerman, R., \& Lynam, D. (2004). Implicit Attitudes as Potential Protection From Risky Sex: Predicting Condom Use With the IAT. Basic and Applied Social Psychology, 26(1998), 227-236.

Dahl, R. E. (2004). Adolescent brain development: A period of vulnerabilities and opportunities - Keynote Address. Annals of the New York Academy of Sciences, 1021, 1-22. https://doi.org/10.1196/ annals.1308.001

De Graaf, H., Vanwesenbeeck, I., \& Meijer, S. (2015). Educational Differences in Adolescents' Sexual Health: A Pervasive Phenomenon in a National Dutch Sample. The Journal of Sex Research, 52(7), 747-757. https://doi.org/10.1080/00224499.2014.945111

De Houwer, J. (2007). A conceptual and theoretical analysis of evaluative conditioning. The Spanish Journal of Psychology, 10, 230-241. https://doi.org/psi/11387416/articulos/SJOP0707220230A.PDF

De Houwer, J. (2011). Evaluative Conditioning. In T. Schachtman \& S. Reilly (Eds.), Associative Learning and Conditioning Theory: Human and Non-Human Applications (pp. 399-416). Oxford: Oxford University Press. https://doi.org/10.1093/acprof

De Houwer, J., Thomas, S., \& Baeyens, F. (2001). Associative Learning of Likes and Dislikes: A Review of 25 Years of Research on Human Evaluative Conditioning. Psychological Bulletin, 126, 853-869. https://doi.org/10.1037//0033-2909.127.6.853

DeSmet, A., Shegog, R., Van Ryckeghem, D., Crombez, G., \& De Bourdeaudhuij, I. (2015). A Systematic Review and Meta-analysis of Interventions for Sexual Health Promotion Involving Serious Digital Games. Games for Health Journal, 4(2), 78-90. https://doi.org/10.1089/g4h.2014.0110

Desmet, A., Thompson, D., Baranowski, T., Palmeira, A., Verloigne, M., \& De Bourdeaudhuij, I. (2016). Is participatory design associated with the effectiveness of serious digital games for healthy lifestyle promotion? A meta-analysis. Journal of Medical Internet Research, 18(4), 1-19. https://doi.org/ 10.2196/jmir.4444

DeSmet, A., Van Ryckeghem, D., Compernolle, S., Baranowski, T., Thompson, D., Crombez, G., ... De Bourdeaudhuij, I. (2014a). A meta-analysis of serious digital games for healthy lifestyle promotion. Preventive Medicine, 69, 95-107. https://doi.org/10.1016/j.ypmed.2014.08.026 
DeSmet, A., Van Ryckeghem, D., Compernolle, S., Baranowski, T., Thompson, D., Crombez, G., ... De Bourdeaudhuij, I. (2014b). A meta-analysis of serious digital games for healthy lifestyle promotion. Preventive Medicine, 69, 95-107. https://doi.org/10.1016/j.ypmed.2014.08.026

Diamond, A. (2013). Executive Functions. Annual Review of Psychology, 64, 135-168. https://doi.org/10.1146/ annurev-psych-113011-143750

Dias, S., Marques, A., Gama, A., \& Martins, M. (2014). HIV Risky Sexual Behaviors and HIV Infection Among Immigrants: A Cross-Sectional Study in Lisbon, Portugal. International Journal of Environmental Research and Public Health, 11(8), 8552-8566. https://doi.org/10.3390/ijerph110808552

Diederich, A., \& Trueblood, J. S. (2018). A dynamic dual process model of risky decision making. Psychological Review, 125(2), 270-292. https://doi.org/10.1037/rev0000087

Dietrich, J., Khunwane, M., Laher, F., de Bruyn, G., Sikkema, K. J., \& Gray, G. (2011). "Group sex" parties and other risk patterns: A qualitative study about the perceptions of sexual behaviours and attitudes of adolescents in Soweto, South Africa. Vulnerable Children and Youth Studies, 6(February 2015), 244-254. https://doi.org/10.1080/17450128.2011.597796

Döring, N. (2014). Consensual sexting among adolescents: Risk prevention through abstinence education or safer sexting? Cyberpsychology: Journal of Psychosocial Research on Cyberspace, 8(1). https://doi.org/ 10.5817/CP2014-1-9

Ducheneaut, N., Wen, M., Yee, N., \& Wadley, G. (2009). Body and Mind: A Study of Avatar Personalization in Three Virtual Worlds. Proceedings of the SIGCHI Conference on Human Factors in Computing Systems, 1151-1 16 0 . Retrieved from http://dl.acm.org/citation.cfm?id=1 5118877

Durlak, J. A., \& DuPre, E. P. (2008). Implementation Matters: A Review of Research on the Influence of Implementation on Program Outcomes and the Factors Affecting Implementation. American Journal of Community Psychology, 41(3-4), 327-350. https://doi.org/10.1007/s10464-008-9165-0

Eagle, D. M., Bari, A., \& Robbins, T. W. (2008). The neuropsychopharmacology of action inhibition: Cross-species translation of the stop-signal and go/no-go tasks. Psychopharmacology, 199, 439-456. https://doi.org/10.1007/s00213-008-1127-6

Ebel-Lam, A. P., MacDonald, T. K., Zanna, M. P., \& Fong, G. T. (2009). An Experimental Investigation of the Interactive Effects of Alcohol and Sexual Arousal on Intentions to Have Unprotected Sex. Basic and Applied Social Psychology, 31(3), 226-233. https://doi.org/10.1080/01973530903058383

Edelmann, R. J. (1985). Social Embarrassment: An Analysis of the Process. Journal of Social and Personal Relationships, 2(2), 195-213.

Eisenberg, M. E., Bernat, D. H., Bearinger, L. H., \& Resnick, M. D. (2008). Support for Comprehensive Sexuality Education: Perspectives from Parents of School-Age Youth. Journal of Adolescent Health, 42(4), 352-359. https://doi.org/10.1016/j.jadohealth.2007.09.019

El-Hilly, A. A., Iqbal, S. S., Ahmed, M., Sherwani, Y., Muntasir, M., Siddiqui, S., ... Eisingerich, A. B. (2016). Game On? Smoking Cessation Through the Gamification of mHealth: A Longitudinal Qualitative Study. JMIR Serious Games, 4(2), e18. https://doi.org/10.2196/games.5678

Enah, C., Moneyham, L., Vance, D. E., \& Childs, G. (2013). Digital Gaming for HIV Prevention With Young Adolescents. Journal of the Association of Nurses in AIDS Care, 24(1), 71-80. https://doi.org/10.1016/ j.jana.2012.03.005

Enge, S., Behnke, A., Fleischhauer, M., Küttler, L., Kliegel, M., \& Strobel, A. (2014). No evidence for true training and transfer effects after inhibitory control training in young healthy adults. Journal of Experimental Psychology: Learning Memory and Cognition, 40, 987-1001. https://doi.org/10.1037/ a0036165

Enock, P. M., Hofmann, S. G., \& McNally, R. J. (2014). Attention bias modification training via smartphone to reduce social anxiety: A randomized, controlled multi-session experiment. Cognitive Therapy and Research, 38(2), 200-216. https://doi.org/10.1007/s10608-014-9606-z

Erhel, S., \& Jamet, E. (2013). Digital game-based learning: Impact of instructions and feedback on motivation and learning effectiveness. Computers \& Education, 67, 156-167. https://doi.org/10.1016/j.compedu. 2013.02.019 
Espy, K. A., McDiarmid, M. M., Cwik, M. F., Stalets, M. M., Hamby, A., \& Senn, T. E. (2004). The contribution of executive functions to emergent mathematic skills in preschool children. Developmental Neuropsychology. https://doi.org/10.1207/s15326942dn2601_6

Evans, J. S. B. T. (2008). Dual-Processing Accounts of Reasoning, Judgment, and Social Cognition. Annual Review of Psychology, 59(1), 255-278. https://doi.org/10.1146/annurev.psych.59.103006.093629

Eysenck, H. J., \& Eysenck, M. W. (1985). Personality and individual differences: A natural science approach. New York: Plenum.

Fathi, A., Hodgins, J. K., \& Rehg, J. M. (2012). Social interactions: A first-person perspective. In Proceedings of the IEEE Computer Society Conference on Computer Vision and Pattern Recognition.

Fentahun, N., Assefa, T., Alemseged, F., \& Ambaw, F. (2012). Parents' perception, students' and teachers' attitude towards school sex education. Ethiopian Journal of Health Sciences, 22(2), 99-106.

Fernández-Romero, J. A., Deal, C., Herold, B. C., Schiller, J., Patton, D., Zydowsky, T., ... Narasimhan, M. (2015). Multipurpose prevention technologies: The future of HIV and STI protection. Trends in Microbiology, 23(7), 429-436. https://doi.org/10.1016/j.tim.2015.02.006

Ferrara, J. (2013). Games for Persuasion: Argumentation, Procedurality, and the Lie of Gamification. Games and Culture, 8(4), 289-304. https://doi.org/10.1177/1555412013496891

Festinger, L. (1957). A theory of cognitive dissonance. Scientific American (Vol. 207). https://doi.org/10.1037/ 10318-001

Field, M., \& Eastwood, B. (2005). Experimental manipulation of attentional bias increases the motivation to drink alcohol. Psychopharmacology, 183(3), 350-357. https://doi.org/10.1007/s00213-005-0202-5

Finer, L. B., \& Philbin, J. M. (2013). Sexual Initiation, Contraceptive Use, and Pregnancy Among Young Adolescents. Pediatrics, 131(5), 886-891. https://doi.org/10.1542/peds.2012-3495

Fischhoff, B., Parker, A. M., Bruine De Bruin W, Downs, J., Palmgren, C., Dawes, R., \& Manski, C. F. (2000). Teen expectations for significant life events. Public Opinion Quarterly, 64(2), 189-205.

Fishbein, M, \& Ajzen, I. (2010). Predicting and changing behaviour: The reasoned action approach. Psychology Press.

Fishbein, M., \& Ajzen, I. (1975). Belief, Attitude, Intention and Behaviour: An Introduction to Theory and Research. Addison-Wesley. New York: Addison-Wesley.

Fishbein, M., \& Ajzen, I. (2010). Predicting and Changing behavior. The Reasoned Action Approach. New York: Psychology Press.

Fleming, D. T., \& Wasserheit, J. N. (1999). From epidemiological synergy to public health policy and practice: the contribution of other sexually transmitted diseases to sexual transmission of HIV infection. Sexually Transmitted Infections, 75(1), 3-17. https://doi.org/10.1136/sti.75.1.3

Fletcher, J., \& Kumar, S. (2014). Religion and risky health behaviors among U.S. adolescents and adults. Journal of Economic Behavior \& Organization, 104, 123-140.

Flood, M. (2003). Lust, trust and latex: Why young heterosexual men do not use condoms. Culture, Health \& Sexuality, 5(4), 353-369. https://doi.org/10.1080/1369105011000028273

Folk, C. L., Remington, R. W., \& Johnston, J. C. (1992). Involuntary covert orienting is contingent on attentional control settings. Journal of Experimental Psychology. Human Perception and Performance, 18(4), 10301044.

Forbes, E. E., \& Dahl, R. E. (2012). Research Review: Altered reward function in adolescent depression: What, when and how? Journal of Child Psychology and Psychiatry and Allied Disciplines, 53(1), 3-15. https://doi.org/10.1111/j.1469-7610.2011.02477.x

Forrest, S., Strange, V., Oakley, A., \& The RIPPLE Study Team. (2004). What do young people want from sex education? The results of a needs assessment from a peer-led sex education programme. Culture, Health \& Sexuality, 6(4), 337-354. https://doi.org/10.1080/13691050310001645050

Forstmann, M., Burgmer, P., \& Mussweiler, T. (2012). "The Mind Is Willing, but the Flesh Is Weak": The Effects of Mind-Body Dualism on Health Behavior. Psychological Science, 23(10), 1239-1245.

Fox, E. (2009). Emotion science. Basingstoke, United Kingdom: Macmillan. 
Frankel, A. S., Bass, S. B., Patterson, F., Dai, T., \& Brown, D. (2018). Sexting, Risk Behavior, and Mental Health in Adolescents: An Examination of 2015 Pennsylvania Youth Risk Behavior Survey Data. Journal of School Health, 88(3), 190-199. https://doi.org/10.1111/josh.12596

French, S. E., \& Holland, K. J. (2013). Condom Negotiation Strategies as a Mediator of the Relationship between Self-Efficacy and Condom Use. Journal of Sex Research, 50(1), 48-59.

Fridlund, V., Stenqvist, K., \& Nordvik, M. K. (2014). Condom use: The discrepancy between practice and behavioral expectations. Scandinavian Journal of Public Health, 42(8), 759-765. https://doi.org/10.1177/ 1403494814550518

Friese, M., \& Hofmann, W. (2009). Control me or I will control you: Impulses, trait self-control, and the guidance of behavior. Journal of Research in Personality, 43(5), 795-805.

Friese, M., Hofmann, W., \& Wänke, M. (2008). When impulses take over: moderated predictive validity of explicit and implicit attitude measures in predicting food choice and consumption behaviour. The British Journal of Social Psychology / the British Psychological Society, 47(Pt 3), 397-419.

Gabler, J., Kropp, F., Silvera, D. H., \& Lavack, A. M. (2004). The role of attitudes and self-efficacy in predicting condom use and purchase intentions. Health Marketing Quarterly, 21(3), 63-78.

Gardner, M., \& Steinberg, L. (2005). Peer influence on risk taking, risk preference, and risky decision making in adolescence and adulthood: an experimental study. Developmental Psychology, 41(4), 625-635. https://doi.org/10.1037/0012-1649.41.4.625

Gawronski, B., \& Bodenhausen, G. V. (2006). Associative and propositional processes in evaluation: an integrative review of implicit and explicit attitude change. Psychological Bulletin, 132(5), 692-731. https://doi.org/10.1037/0033-2909.132.5.692

Gawronski, B., Hofmann, W., \& Wilbur, C. J. (2006). Are "implicit" attitudes unconscious? Consciousness and Cognition.

Gerrard, M., Gibbons, F. X., Houlihan, A. E., Stock, M. L., \& Pomery, E. A. (2008). A dual-process approach to health risk decision making: The prototype willingness model. Developmental Review, 28(1), $29-61$. https://doi.org/10.1016/j.dr.2007.10.001

Gibbons, F. X., Gerrard, M., Blanton, H., \& Russell, D. W. (1998). Reasoned action and social reaction: Willingness and intention as independent predictors of health risk. Journal of Personality and Social Psychology, 74(5), 1164-1180. https://doi.org/10.1037/0022-3514.74.5.1164

Gibson, B. (2008). Can Evaluative Conditioning Change Attitudes toward Mature Brands? New Evidence from the Implicit Association Test. Journal of Consumer Research, 35(1), 178-188. https://doi.org/10.1086/ 527341

Gillman, A. S., Yeater, E. A., Feldstein Ewing, S. W., Kong, A. S., \& Bryan, A. D. (2018). Risky Sex in High-Risk Adolescents: Associations with Alcohol Use, Marijuana Use, and Co-Occurring Use. AIDS and Behavior, 22(4), 1352-1362. https://doi.org/10.1007/s10461-017-1900-9

Girard, C., Ecalle, J., \& Magnan, A. (2013). Serious games as new educational tools: how effective are they? A meta-analysis of recent studies. Journal of Computer Assisted Learning, 29(3), 207-219. https://doi.org/10.1111/j.1365-2729.2012.00489.x

Globerman, J., Mitra, S., Gogolishvili, D., Rueda, S., Schoffel, L., Gangbar, K., ... Rourke, S. B. (2017). HIV/STI prevention interventions: A systematic review and meta-analysis. Open Medicine, 12(1), 450-467. https://doi.org/10.1515/med-2017-0064

Göbel, S. (2016). Serious Games Application Examples. In R. Dörner, S. Göbel, \& W. Effelsberger (Eds.), Serious Games. Springer. https://doi.org/10.1007/978-3-319-40612-1_12

Goldfarb, E., \& Constantine, N. (2011). Sexuality education. In Encyclopedia of adolescence normative proceses in development (pp. 322-331). San Diego, Academic.

Gomm, R. (2009). Key concepts in in social research methods. London: Palgrave Macmillan.

Gore, F. M., Bloem, P. J. N., Patton, G. C., Ferguson, J., Joseph, V., Coffey, C., ... Mathers, C. D. (2011). Global burden of disease in young people aged 10-24 years: A systematic analysis. The Lancet, 377(9783), 20932102. 
Graesser, A., Chipman, P., Leeming, F., \& Biedenbach, S. (2009). Deep learning and emotion in serious games. In U. Ritterfeld, M. Cody, \& P. Vorderer (Eds.), Serious Games, Mechanisms and Effects. New York: Routledge.

Grauvogl, A., de Jong, P., Peters, M., Evers, S., van Overveld, M., \& van Lankveld, J. (2015). Disgust and sexual arousal in young adult men and women. Archives of Sexual Behavior, 44, 1515-1525. https://doi.org/10.1007/s10508-014-0349-4

Gray, H. M., Ambady, N., Lowenthal, W. T., \& Deldin, P. (2004). P300 as an index of attention to self-relevant stimuli. Journal of Experimental Social Psychology, 40(2), 216-224.

Greene, K., Krcmar, M., Walters, L. H., Rubin, D. L., Jerold, \& Hale, L. (2000). Targeting adolescent risk-taking behaviors: the contributions of egocentrism and sensation-seeking. Journal of Adolescence, 23(4), 439461.

Greenwald, A. G., \& Banaji, M. R. (1995). Implicit Social Cognition: Attitudes, Self-Esteem, and Stereotypes. Psychological Review, 102(1), 4-27.

Greenwald, A. G., McGhee, D. E., \& Schwartz, J. L. (1998). Measuring individual differences in implicit cognition: the implicit association test. Journal of Personality and Social Psychology, 74(6), 1464-1480. https://doi.org/10.1037/0022-3514.74.6.1464

Greenwald, A. G., Nosek, B. A., \& Banaji, M. R. (2003). Understanding and using the implicit association test: I. An improved scoring algorithm. Journal of Personality and Social Psychology, 85(2), 197-216.

Greenwald, A. G., PoehIman, T. A., UhImann, E. L., \& Banaji, M. R. (2009). Understanding and using the Implicit Association Test: III. Meta-analysis of predictive validity. Journal of Personality and Social Psychology, 97(1), 17-41. https://doi.org/10.1037/a0015575

Grenard, J. L., Ames, S. L., \& Stacy, A. W. (2013). Deliberative and spontaneous cognitive processes associated with HIV risk behavior. Journal of Behavioral Medicine, 36(1), 95-107.

Grenard, J. L., Ames, S. L., Wiers, R. W., Thush, C., Sussman, S., \& Stacy, A. W. (2008). Working memory capacity moderates the predictive effects of drug-related associations on substance use. Psychology of Addictive Behaviors. https://doi.org/10.1037/0893-164X.22.3.426

Gross, J. J., \& Barrett, L. F. (2013). The emerging field of affective science. Emotion, 13(6), 997-998. https://doi.org/10.1037/a0034512

Grossman, J. M., Tracy, A. J., \& Noonan, A. E. (2013). Adolescents' religious discordance with mothers: Is there a connection to sexual risk behavior during emerging adulthood? Journal of Primary Prevention, 34(5), 329-343. https://doi.org/10.1007/s10935-013-0315-2

Gullette, D. L., \& Lyons, M. A. (2005). Sexual Sensation Seeking, Compulsivity, and HIV Risk Behaviors in College Students. Journal of Community Health Nursing, 22(1), 47-60. https://doi.org/10.1207/ s15327655jchn2201_5

GUO, J., CHUNG, I., HILL, K., HAWKINS, J., CATALANO, R., \& ABBOTT, R. (2002). Developmental relationships between adolescent substance use and risky sexual behavior in young adulthood. Journal of Adolescent Health, 31(4), 354-362. https://doi.org/10.1016/S1054-139X(02)00402-0

Guo, R., McAleese, W. J., Appleby, K. M., Guo, J., Zhang, W., Huang, Y., \& Peterson, T. (2014). Predictors of Intention to Use Condoms Among Chinese College Students. Journal of Community Health, 39, 712-718.

Guye, S., \& von Bastian, C. C. (2017). Working memory training in older adults: Bayesian evidence supporting the absence of transfer. Psychology and Aging, 32(8), 732-746. https://doi.org/10.1037/pag0000206

Haddad, A. D. M., Harrison, F., Norman, T., \& Lau, J. Y. F. (2014). Adolescent and adult risk-taking in virtual social contexts. Frontiers in Psychology, 5. https://doi.org/10.3389/fpsyg.2014.01476

Hahm, H. C., Lee, J., Rough, K., \& Strathdee, S. A. (2012). Gender power control, sexual experiences, safer sex practices, and potential HIV risk behaviors among young Asian-American women. AIDS and Behavior, 16(1), 179-188.

Hahn, A., \& Gawronski, B. (2014). Do implicit evaluations reflect unconscious attitudes? Behavioral and Brain Sciences, 37(1), 28-29.

Halpern-Felsher, B. (2008). Oral Sexual Behavior: Harm Reduction or Gateway Behavior? Journal of Adolescent Health, 43(3), 207-208. 
Halpern-Felsher, B. L., Cornell, J. L., Kropp, R. Y., \& Tschann, J. M. (2005). Oral versus vaginal sex among adolescents: perceptions, attitudes, and behavior. Pediatrics, 115(4), 845-851.

Hamari, J., Koivisto, J., \& Sarsa, H. (2014). Does Gamification Work?--A Literature Review of Empirical Studies on Gamification. System Sciences (HICSS), 2014 47th Hawaii International Conference On, 3025-3034.

Harper, G. W. (2007). Sex isn't that simple: culture and context in HIV prevention interventions for gay and bisexual male adolescents. The American Psychologist, 62(8), 803-819.

Heeren, A., Mogoase, C., Philippot, P., \& McNally, R. J. (2015). Attention bias modification for social anxiety: A systematic review and meta-analysis. Clinical Psychology Review, 40, 76-90. https://doi.org/10.1016/ j.cpr.2015.06.001

Helmerhorst, H. J., Brage, S., Warren, J., Besson, H., \& Ekelund, U. (2012). A systematic review of reliability and objective criterion-related validity of physical activity questionnaires. International Journal of Behavioral Nutrition and Physical Activity, 9(1), 103. https://doi.org/10.1186/1479-5868-9-103

Helweg-Larsen, M., \& Collins, B. E. (1994). The UCLA Multidimensional Condom Attitudes Scale: Documenting the complex determinants of condom use in college students. Health Psychology, 13(3), 224-237. https://doi.org/10.1037/0278-6133.13.3.224

Henderson, M., Wight, D., Raab, G., Abraham, C., Buston, K., Hart, G., \& Scott, S. (2002). Heterosexual risk behaviour among young teenagers in Scotland. Journal of Adolescence, 25(5), 483-494. https://doi.org/ 10.1006/jado.2002.0493

Higgins, J. A., \& Hirsch, J. S. (2008). Pleasure, Power, and Inequality: Incorporating Sexuality Into Research on Contraceptive Use. American Journal of Public Health, 98(10), 1803-1813. https://doi.org/10.2105/AJPH. 2007.115790

Higgins, J. A., \& Wang, Y. (2015). The Role of Young Adults' Pleasure Attitudes in Shaping Condom Use. American Journal of Public Health, 105(7), 1329-1332. https://doi.org/10.2105/AJPH.2015.302567

Hightow-Weidman, L. B., Muessig, K. E., Bauermeister, J. A., LeGrand, S., \& Fiellin, L. E. (2017). The future of digital games for HIV prevention and care. Current Opinion in HIV and AIDS, 12(5), 501-507. https://doi.org/10.1097/COH.0000000000000399

Hill, S. E., \& Durante, K. M. (2011a). Courtship, competition, and the pursuit of attractiveness: mating goals facilitate health-related risk taking and strategic risk suppression in women. Personality and Social Psychology Bulletin, 37(3), 383-394. https://doi.org/10.1177/0146167210395603

Hill, S. E., \& Durante, K. M. (2011b). Courtship, competition, and the pursuit of attractiveness: mating goals facilitate health-related risk taking and strategic risk suppression in women. Personality and Social Psychology Bulletin, 37(3), 383-394.

Hingson, R. W., Strunin, L., Berlin, B. M., \& Heeren, T. (1990). Beliefs about AIDS, use of alcohol and drugs, and unprotected sex among Massachusetts adolescents. American Journal of Public Health, 80(3), 295-299. https://doi.org/10.2105/AJPH.80.3.295

Hipwell, A. E., Stepp, S. D., Keenan, K., Chung, T., \& Loeber, R. (2011). Brief report: Parsing the heterogeneity of adolescent girls' sexual behavior: Relationships to individual and interpersonal factors. Journal of Adolescence, 34(3), 589-592. https://doi.org/10.1016/j.adolescence.2010.03.002

Hofmann, W., De Houwer, J., Perugini, M., Baeyens, F., \& Crombez, G. (2010a). Evaluative conditioning in humans: a meta-analysis. Psychological Bulletin, 136(3), 390-421. https://doi.org/10.1037/a0018916

Hofmann, W., De Houwer, J., Perugini, M., Baeyens, F., \& Crombez, G. (2010b). Evaluative Conditioning in Humans: A Meta-Analysis. Psychological Bulletin, 136(3), 390-421. https://doi.org/10.1037/a0018916

Hofmann, W., Friese, M., \& Strack, F. (2009). Impulse and self-control from a dual-systems perspective. Perspectives on Psychological Science, 4(2), 1-15. https://doi.org/10.1111/j.1745-6924.2009.01116.x

Hofmann, W., Friese, M., \& Wiers, R. (2008). Impulsive Versus Reflective Influences on Health Behavior: A Theoretical Framework and Empirical Review. Health Psychology Review. https://doi.org/10.1080/1743 7190802617668

Hofmann, W., Gschwendner, T., Friese, M., Wiers, R. W., \& Schmitt, M. (2008). Working memory capacity and self-regulatory behavior: toward an individual differences perspective on behavior determination by automatic versus controlled processes. Journal of Personality and Social Psychology, 95(4), 962-977. https://doi.org/10.1037/a0012705 
Hollingsworth, T. D., Anderson, R. M., \& Fraser, C. (2008). HIV-1 transmission, by stage of infection. The Journal of Infectious Diseases, 198(5), 687-693. https://doi.org/10.1086/590501

Holway, G. V., Tillman, K. H., \& Brewster, K. L. (2017). Binge Drinking in Young Adulthood: The Influence of Age at First Intercourse and Rate of Sex Partner Accumulation. Archives of Sexual Behavior, 46(2), 525-537. https://doi.org/10.1007/s10508-015-0597-y

Horcajo, J., Rubio, V. J., Aguado, D., Hernández, J. M., \& Márquez, M. O. (2 $\left.\begin{array}{llll}2 & 1 & 4\end{array}\right)$. Using the implicit association test to assess risk propensity self-concept: Analysis of its predictive validity on a risk-taking behaviour in a natural setting. European Journal of Personality, 28(5), 459-471. https://doi.org/10.1002/per.1925

Horvath, K. J., Rosser, B. R. S., \& Remafedi, G. (2008). Sexual risk taking among young internet-using men who have sex with men. American Journal of Public Health, 98(6), 1059-1067. https://doi.org/10.2105/ AJPH.2007.111070

Houben, K., Havermans, R. C., Nederkoorn, C., \& Jansen, A. (2012). Beer à no-go: learning to stop responding to alcohol cues reduces alcohol intake via reduced affective associations rather than increased response inhibition. Addiction, 107(7), 1280-1287. https://doi.org/10.1111/j.1360-0443.2012.03827.x

Houben, K., Nederkoorn, C., Wiers, R. W., \& Jansen, A. (2011). Resisting temptation: Decreasing alcohol-related affect and drinking behavior by training response inhibition. Drug and Alcohol Dependence, 116(1-3), 132136. https://doi.org/10.1016/j.drugalcdep.2010.12.011

Houdé, O., \& Borst, G. (2015). Evidence for an inhibitory-control theory of the reasoning brain. Frontiers in Human Neuroscience. https://doi.org/10.3389/fnhum.2015.00148

Houlihan, S. (2018). Dual-process models of health-related behaviour and cognition: a review of theory. Public Health, 156, 52-59. https://doi.org/10.1016/j.puhe.2017.11.002

House, L. D., Mueller, T., Reininger, B., Brown, K., \& Markham, C. M. (2010). Character as a Predictor of Reproductive Health Outcomes for Youth: A Systematic Review. Journal of Adolescent Health, 46(3 SUPPL.), S59-S74. https://doi.org/10.1016/j.jadohealth.2009.11.218

Hoyle, R. H., Stephenson, M. T., Palmgreen, P., Lorch, E. P., \& Donohew, R. L. (2002). Reliability and validity of a brief measure of sensation seeking. Personality and Individual Differences, 32(3), 401-414.

Huebner, A., \& Howell, L. (2003). Examining the relationship between adolescent sexual risk-taking and perceptions of monitoring, communication, and parenting styles. Journal of Adolescent Health, 33(2), 7178. https://doi.org/10.1016/S1054-139X(03)00141-1

Hughey, M. (1998). New Tribalisms. London: Palgrave Macmillan.

Hyde, A., Drennan, J., Butler, M., Howlett, E., Carney, M., \& Lohan, M. (2013). Parents' constructions of communication with their children about safer sex. Journal of Clinical Nursing, 22(23-24), 3438-3446. https://doi.org/10.1111/jocn.12367

Hyde, A., Drennan, J., Howlett, E., \& Brady, D. (2008). Safer heterosex: perspectives from young men in Ireland. Sexual Health, 5(1), 25. https://doi.org/10.1071/SH07062

Imhoff, R., \& Schmidt, A. F. (2014). Sexual disinhibition under sexual arousal: evidence for domain specificity in men and women. Archives of Sexual Behavior, 43(6), 1123-1136.

Ito, T. A., Larsen, J. T., Smith, N. K., \& Cacioppo, J. T. (1998). Negative information weighs more heavily on the brain: the negativity bias in evaluative categorizations. Journal of Personality and Social Psychology, 75(4), 887-900.

Jaccard, J., \& Blanton, H. (2007). A theory of implicit reasoned action: The role of implicit and explicit attitudes in the prediction of behavior. Prediction and Change of Health Behavior: Applying the Reasoned Action Approach. https://doi.org/10.4324/9780203937082

Jacques, S., \& Marcovitch, S. (2010). Development of Executive Function across the Life Span. In The Handbook of Life-Span Development. https://doi.org/10.1002/9780470880166.hlsd001013

Jaffard, M., Benraiss, A., Longcamp, M., Velay, J. L., \& Boulinguez, P. (2007). Cueing method biases in visual detection studies. Brain Research. https://doi.org/10.1016/j.brainres.2007.08.032

Jaffard, M., Longcamp, M., Velay, J. L., Anton, J. L., Roth, M., Nazarian, B., \& Boulinguez, P. (2008). Proactive inhibitory control of movement assessed by event-related fMRI. Neurolmage. https://doi.org/10.1016/ j.neuroimage.2008.05.041 
Janepanish, P., Dancy, B. L., \& Park, C. (2011). Consistent condom use among Thai heterosexual adult males in Bangkok, Thailand. AIDS Care, 23(4), 460-466.

Janssen, E., Everaerd, W., Spiering, M., \& Janssen, J. (2000). Automatic processes and the appraisal of sexual stimuli: Toward an information processing model of sexual arousal. Journal of Sex Research, 37(1), 8-23.

Janssen, E., Prause, N., \& Geer, J. H. (2007). The sexual response. In J. T. Cacioppo, L. G. Tassinary, \& G. G. Berntson (Eds.), Handbook of psychophysiology (3rd ed.). (pp. 245-266). Cambridge University Press.

Johns, G., \& Miraglia, M. (2015). The reliability, validity, and accuracy of self-reported absenteeism from work: A meta-analysis. Journal of Occupational Health Psychology, 20(1), 1-14. https://doi.org/10.1037/ a0037754

Johnson, R. (1993). On the neural generators of the P300 component of the event-related potential. Psychophysiology, 30(1), 90-97.

Jones, B. T., Jones, B. C., Thomas, A. P., \& Piper, J. (2003). Alcohol consumption increases attractiveness ratings of opposite-sex faces: A possible third route to risky sex. Addiction, 98(8), 1069-1075.

Jones, C. R., Olson, M. A., \& Fazio, R. H. (2010). Evaluative Conditioning. The "How" Question. Advances in Experimental Social Psychology, 43, 205-255. https://doi.org/10.1016/S0065-2601(10)43005-1

Jones, D. J., Olson, A. L., Forehand, R., Gaffney, C. A., Zens, M. S., \& Bau, J. J. (2005). A family-focused randomized controlled trial to prevent adolescent alcohol and tobacco use: The moderating roles of positive parenting and adolescent gender. Behavior Therapy, 36(4), 347-355.

Jonides, J., \& Mack, R. (1984). On the cost and benefit of cost and benefit. Psychological Bulletin, 96(1), 29-44. https://doi.org/10.1037//0033-2909.96.1.29

Kachur, R., Mesnick, J., Liddon, N., Kapsimalis, C., Habel, M., David-Ferdon, C., Brown, K., Gloppen, K., Tevendale, H., Gelaude, D.J., Romero, L., Seitz, H., H., A. B., Schindelar, J., Mesnick, Liddon, Kapsimalis, ... Schindelar. (2013). Adolescents, Technology and Reducing Risk for HIV, STDs and Pregnancy. Atlanta. Retrieved from https://www.cdc.gov/std/life-stages-populations/Adolescents-white-paper.pdf

Kakoschke, N., Kemps, E., \& Tiggemann, M. (2014). Attentional bias modification encourages healthy eating. Eating Behaviors, 15(1), 120-124. https://doi.org/10.1016/j.eatbeh.2013.11.001

Kankaanranta, M., \& Neittaanmki, P. (2009). Design and Use of Serious Games. Engineering (Vol. 37). Amsterdam: Springer.

Kapp, K. (2012). The Gamification of Learning and Instruction: Game-Based Methods and Strategies For Training And Education. San Francisco: John Wiley \& Sons.

Karpinski, A., \& Steinman, R. B. (2006). The single category implicit association test as a measure of implicit social cognition. Journal of Personality and Social Psychology, 91(1), 16-32. https://doi.org/10.1037/00223514.91.1.16

Kato, P. M. (2010). Video games in health care: Closing the gap. Review of General Psychology, 14(2), $113-121$.

Kato, P. M., Cole, S. W., Bradlyn, A. S., \& Pollock, B. H. (2008). A Video Game Improves Behavioral Outcomes in Adolescents and Young Adults With Cancer: A Randomized Trial. PEDIATRICS, 122(2), e305-e317. https://doi.org/10.1542/peds.2007-3134

Keltner, D., \& Buswell, B. N. (1997). Embarrassment: its distinct form and appeasement functions. Psychological Bulletin, 122(3), 250-270. Retrieved from http://www.ncbi.nlm.nih.gov/pubmed/9354148

Keren, G., \& Schul, Y. (2009). Two Is Not Always Better Than One. Perspectives on Psychological Science, 4(6), 533-550. https://doi.org/10.1111/j.1745-6924.2009.01164.x

Kessels, L. T. E., Ruiter, R. a C., \& Jansma, B. M. (2010). Increased attention but more efficient disengagement: neuroscientific evidence for defensive processing of threatening health information. Hea Ith Psych olog $y$ : Official Journal of the Division of Health Psychology, American Psychological Association, 29(4), 346-354. https://doi.org/10.1037/a0019372

Khurana, A., Romer, D., Betancourt, L. M., Brodsky, N. L., Giannetta, J. M., \& Hurt, H. (2012). Early adolescent sexual debut: The mediating role of working memory ability, sensation seeking, and impulsivity. Developmental Psychology, 48(5), 1416-1428. https://doi.org/10.1037/a0027491

Kim, J. H., \& Koopman, J. S. (2012). HIV transmissions by stage in dynamic sexual partnerships. Journal of Theoretical Biology, 298, 147-153. https://doi.org/10.1016/j.jtbi.2011.12.021 
Kincaid, C., Jones, D. J., Sterrett, E., \& McKee, L. (2012). A review of parenting and adolescent sexual behavior: The moderating role of gender. Clinical Psychology Review, 32(3), 177-188. https://doi.org/10.1016/ j.cpr.2012.01.002

Kirby, D. B. (2008). The impact of abstinence and comprehensive sex and STD/HIV education programs on adolescent sexual behavior. Sexuality Research and Social Policy. https://doi.org/10.1525/srsp.2008. 5.3.18

Kirby, D. B., \& Laris, B. a. (2009). Effective curriculum-based sex and STD/HIV education programs for adolescents. Child Development Perspectives, 3(1), 21-29. https://doi.org/10.1111/j.1750-8606. 2008.00071.x

Kirby, D. B., Laris, B. a., \& Rolleri, L. a. (2007). Sex and HIV Education Programs: Their Impact on Sexual Behaviors of Young People Throughout the World. Journal of Adolescent Health, 40(3), 206-217. https://doi.org/10.1016/j.jadohealth.2006.11.143

Kline, P. (1993). The handbook of psychological testing (2nd ed.). London: Routledge.

Klingberg, T. (2010). Training and plasticity of working memory. Trends in Cognitive Sciences, 14, 317-324. https://doi.org/10.1016/j.tics.2010.05.002

Klingberg, T., Forssberg, H., \& Westerberg, H. (2002a). Training of working memory in children with ADHD. Journal of Clinical and Experimental Neuropsychology, 24(6), 781-791.

Klingberg, T., Forssberg, H., \& Westerberg, H. (2002b). Training of Working Memory in Children With ADHD. Journal of Clinical and Experimental Neuropsychology. https://doi.org/10.1076/jcen.24.6.781.8395

Kohler, P. K., Manhart, L. E., \& Lafferty, W. E. (2008). Abstinence-Only and Comprehensive Sex Education and the Initiation of Sexual Activity and Teen Pregnancy. Journal of Adolescent Health, 42(4), 344-351.

Kok, A. (1997). Event-related-potential (ERP) reflections of mental resources: A review and synthesis. Biological Psychology, 45(1-3), 19-56.

Koning, I. M., Maric, M., MacKinnon, D., \& Vollebergh, W. A. M. (2015). Effects of a Combined Parent-Student Alcohol Prevention Program on Intermediate Factors and Adolescents' Drinking Behavior: A Sequential Mediation Model. Journal of Consulting and Clinical Psychology. Retrieved from http://www.ncbi.nlm.nih.gov/ pubmed/25939019

Kosenko, K., Luurs, G., \& Binder, A. R. (2017). Sexting and Sexual Behavior, 2011-2015: A Critical Review and Meta-Analysis of a Growing Literature. Journal of Computer-Mediated Communication, 22(3), 141-160. https://doi.org/10.1111/jcc4.12187

Kotchick, B. A., Shaffer, A., Forehand, R., \& Miller, K. S. (2001). Adolescent sexual risk behavior: a multi-system perspective. Clinical Psychology Review, 21(4), 493-519. https://doi.org/10.1016/S0272-7358(99)00070-7

Krug, R., Plihal, W., Fehm, H. L., \& Born, J. (2000). Selective influence of the menstrual cycle on perception of stimuli with reproductive significance: An event-related potential study. Psychophysiology. https://doi.org/10.1111/1469-8986.3710111

Kunda, Z. (1990). The case for motivated reasoning. Psychological Bulletin, 108(3), 480-498.

Ladapo, J. a., Elliott, M. N., Bogart, L. M., Kanouse, D. E., Vestal, K. D., Klein, D. J., ... Schuster, M. a. (2013). Cost of Talking Parents, Healthy Teens: A worksite-based intervention to promote parent-adolescent sexual health communication. Journal of Adolescent Health, 53(5), 595-601. https://doi.org/10.1016/ j.jadohealth.2012.11.015

Lakon, C. M., \& Hipp, J. R. (2014). On Social and Cognitive Influences: Relating Adolescent Networks, Generalized Expectancies, and Adolescent Smoking. PLOS ONE, 9(12), e115668. https://doi.org/10.1371/ journal.pone.0115668

Landor, A., Simons, L. G., Simons, R. L., Brody, G. H., \& Gibbons, F. X. (2011). The Role of Religiosity in the Relationship Between Parents, Peers, and Adolescent Risky Sexual Behavior. Journal of Youth and Adolescence, 40(3), 296-309. https://doi.org/10.1007/s10964-010-9598-2

Langlais, M. R., \& Schwanz, S. J. (2018). Centrality of Religiosity of Relationships for Affectionate and Sexual Behaviors Among Emerging Adults. Sexuality \& Culture, 22(2), 405-421. https://doi.org/10.1007/s12119017-9474-2 
Lansford, J. E., Yu, T., Erath, S. a., Pettit, G. S., Bates, J. E., \& Dodge, K. a. (2010). Developmental Precursors of number of sexual partners from ages 16 to 22. Journal of Research on Adolescence, 20(3), 651-677. https://doi.org/10.1111/j.1532-7795.2010.00654.x

LaSala, M. C. (2007). Parental influence, gay youths, and safer sex. Health and Social Work, 32(1), 49-55. https://doi.org/10.1093/hsw/32.1.49

Lazarov, A., \& Bar-Haim, Y. (2016). Attention Bias Modification Treatment Reduces Depression Symptoms in Youth. Journal of the American Academy of Child and Adolescent Psychiatry, 55(3), 161-162. https://doi.org/10.1016/j.jaac.2015.12.009

Levey, A. B., \& Martin, I. (1975). Classical conditioning of human "evaluative" responses. Behaviour Research and Therapy, 13(4), 221-226. https://doi.org/10.1016/0005-7967(75)90026-1

Li, Y., Cottrell, R. R., Wagner, D. I., \& Ban, M. (2004). Needs and preferences regarding sex education among Chinese college students: a preliminary study. International Family Planning Perspectives, 30(3), 128-133.

Liberman, A., \& Chaiken, S. (1992). Defensive Processing of Personally Relevant Health Messages. Personality and Social Psychology Bulletin, 18(6), 669-679.

Lindgren, R. (2013). Examining the effects of avatar customization and narrative on engagement and learning in video games. In Proceedings of CGAMES 2013 USA - 18th International Conference on Computer Games: Al, Animation, Mobile, Interactive Multimedia, Educational and Serious Games (pp. 87-90). https://doi.org/10.1109/CGames.2013.6632611

Livingstone, S., \& Görzig, A. (2014). When adolescents receive sexual messages on the internet: Explaining experiences of risk and harm. Computers in Human Behavior, 33, 8-15. https://doi.org/10.1016/ j.chb.2013.12.021

Loewenstein, G., Nagin, D., \& Paternoster, R. (1997). The Effect of Sexual Arousal on Expectations of Sexual Forcefulness. Journal of Research in Crime and Delinquency, 34(4), 443-473.

Logan, G. D. (1994). On the ability to inhibit thought and action: A users' guide to the stop signal paradigm. In Inhibitory processes in attention, memory, and language. https://doi.org/10.1016/j.jsat.2006.09.008

Logan, G. D., \& Cowan, W. B. (1984). On the ability to inhibit thought and action: A theory of an act of control. Psychological Review. https://doi.org/10.1037/0033-295X.91.3.295

Longest, K. C., \& Uecker, J. E. (2018). Moral Communities and Sex: The Religious Influence on Young Adult Sexual Behavior and Regret. Sociological Perspectives, 61(3), 361-382. https://doi.org/10.1177/07311 21417730015

Lowry, R., Holtzman, D., Truman, B. I., Kann, L., Collins, J. L., \& Kolbe, L. J. (1994). Substance use and HIV-related sexual behaviors among US high school students: are they related? American Journal of Public Health, 84(7), 1116-1120. https://doi.org/10.2105/AJPH.84.7.1116

Lu, A. S., Baranowski, T., Thompson, D., \& Buday, R. (2012). Story Immersion of Videogames for Youth Health Promotion: A Review of Literature. Games for Health Journal, 1(3), 199-204. https://doi.org/10.1089/ g4h.2011.0012

Ma, W. J., Husain, M., \& Bays, P. M. (2014). Changing concepts of working memory. Nature Neuroscience, 17(3), 347-356.

MacDonald, T. K., Fong, G. T., Zanna, M. P., \& Martineau, a M. (2000). Alcohol myopia and condom use: can alcohol intoxication be associated with more prudent behavior? Journal of Personality and Social Psychology, 78(4), 605-619. https://doi.org/10.1037/0022-3514.78.4.605

MacDonald, T. K., Zanna, M. P., \& Fong, G. T. (1996). Why Common Sense Goes Out the Window: Effects of Alcohol on Intentions to Use Condoms. Personality and Social Psychology Bulletin, 22(8), 763-775.

Madkour, A. S., Farhat, T., Halpern, C. T., Godeau, E., \& Gabhainn, S. N. (2010). Early adolescent sexual initiation as a problem behavior: A comparative study of five nations. Journal of Adolescent Health, 47(4), 389-398. https://doi.org/10.1016/j.jadohealth.2010.02.008

Manlove, J., Ryan, S., \& Franzetta, K. (2004). Contraceptive use and consistency in US teenagers' most recent sexual relationships. Perspectives on Sexual and Reproductive Health, 36(6), 265-275.

Manlove, J., \& Terry-Humen, E. (2007). Contraceptive use patterns within females' first sexual relationships: the role of relationships, partners, and methods. Journal of Sex Research, 44(1), 3-16.

March, G. M. (2013). Handbook of Organizations. New York: Routledge. 
Marjoribanks, K. (1997). Family background, social and academic capital, and adolescents' aspriations: A mediational analysis. Social Psychology of Education, 2(2), 177-197.

Markham, C. M., Lormand, D., Gloppen, K. M., Peskin, M. F., Flores, B., Low, B., \& House, L. D. (2010). Connectedness as a Predictor of Sexual and Reproductive Health Outcomes for Youth. Journal of Adolescent Health, 46(3 SUPPL.), S23-S41. https://doi.org/10.1016/j.jadohealth.2009.11.214

Marteau, T. M., Hollands, G. J., \& Fletcher, P. C. (2012). Changing Human Behavior to Prevent Disease: The Importance of Targeting Automatic Processes. Science, 337(6101), 1492-1495. https://doi.org/10.1126/ science. 1226918

Martey, R. M., Stromer-Galley, J., Banks, J., Wu, J., \& Consalvo, M. (2014). The strategic female: genderswitching and player behavior in online games. Information, Communication \& Society, 17(3), 286-300.

Martin, I., \& Levey, A. B. (1978). Evaluative conditioning. Advances in Behaviour Research and Therapy, 1, 57102. https://doi.org/10.1016/0146-6402(78)90013-9

McCambridge, S. a, \& Consedine, N. S. (2014). For whom the bell tolls: experimentally-manipulated disgust and embarrassment may cause anticipated sexual healthcare avoidance among some people. Emotion, 14(2), 407-415.

McEachan, R. R. C., Conner, M., Raylor, N. J., \& Lawton, R. J. (2011). Prospective prediction of health-related behaviors with the Theory of Planned Behavior: a meta-analysis. Health Psychology Review, 5, 97-144.

Measor, L. (2004). Young people's views of sex education: gender, information and knowledge. Sex Education, $4(2), 153-166$.

Mehta, C. M., Sunner, L. E., Head, S., Crosby, R., \& Shrier, L. a. (2011). " Sex Isn't Something You Do with Someone You Don't Care About": Young Women's Definitions of Sex. Journal of Pediatric and Adolescent Gynecology, 24(5), 267-271. https://doi.org/10.1016/j.jpag.2011.03.003

Melendez, R. M., Hoffman, S., Exner, T., Leu, C.-S., \& Ehrhardt, A. a. (2003). Intimate partner violence and safer sex negotiation: effects of a gender-specific intervention. Archives of Sexual Behavior, 32(6), 499-511.

Mello, S., \& Hovick, S. R. (2016). Predicting Behaviors to Reduce Toxic Chemical Exposures Among New and Expectant Mothers: The Role of Distal Variables Within the Integrative Model of Behavioral Prediction. Health Education \& Behavior, 43(6), 705-715. https://doi.org/10.1177/1090198116637600

Mevissen, F. E. F., van Empelen, P., Watzeels, A., van Duin, G., Meijer, S., van Lieshout, S., \& Kok, G. (2018). Development of Long Live Love +, a school-based online sexual health programme for young adults. An intervention mapping approach. Sex Education, 18(1), 47-73. https://doi.org/10.1080/14681811.2017. 1389717

Michael, D. R., \& Chen, S. L. (2005). Serious Games: Games That Educate, Train, and Inform. New York: Muska \& Lipman.

Michaelson, L., de la Vega, A., Chatham, C. H., \& Munakata, Y. (2013). Delaying gratification depends on social trust. Frontiers in Psychology, 4(June), 355. https://doi.org/10.3389/fpsyg.2013.00355

Milhausen, R. R., McKay, A., Graham, C. A., Sanders, S. A., Crosby, R. A., Yarber, W. L., \& Wood, J. (2018). Do Associations Between Pleasure Ratings and Condom Use During Penile-Vaginal Intercourse Vary by Relationship Type?: A Study of Canadian University Students. The Journal of Sex Research, 55(1), 21-30. https://doi.org/10.1080/00224499.2017.1298713

Mischel, W., \& Shoda, Y. (1995). A cognitive-affective system theory of personality: reconceptualizing situations, dispositions, dynamics, and invariance in personality structure. Psychological Review, 102(2), 246-268.

Miyake, A., \& Friedman, N. P. (2012). The nature and organization of individual differences in executive functions: Four general conclusions. Current Directions in Psychological Science, 21, 8-14. https://doi.org/10.1177/0963721411429458

Miyake, A., Friedman, N. P., Emerson, M. J., Witzki, A. H., Howerter, A., \& Wager, T. D. (2000). The Unity and Diversity of Executive Functions and Their Contributions to Complex "Frontal Lobe" Tasks: A Latent Variable Analysis. Cognitive Psychology. https://doi.org/10.1006/cogp.1999.0734

Mogg, K., Bradley, B. P., De Bono, J., \& Painter, M. (1997). Time course of attentional bias for threat information in non-clinical anxiety. Behaviour Research and Therapy, 35(4), 297-303. 
Mogoaşe, C., David, D., \& Koster, E. H. W. ( $\left.\begin{array}{llll}2 & 0 & 1 & 4\end{array}\right)$. Clinical efficicattentional bias modification procedures: An updated meta-analysis. Journal of Clinical Psychology, 70(12), 1133-1157. https://doi.org/10.1002/ jclp.22081

Montaño, D. E., \& Kasprzyk, D. (2008). Theory of Reasoned Action, Theory of Planned Behavior, and the Integrated Behavioral Model. In K. Glanz, B. K. Rimer, \& K. Viswanath (Eds.), Health Behavior and Health Education: Theory, Research and Practic (pp. 67-96). London: Jossey-Bass Publishers.

Moore, S. G., Dahl, D. W., Gorn, G. J., \& Weinberg, C. B. (2006). Coping with condom embarrassment. Psychology, Health \& Medicine, 11(1), 70-79.

Moore, S. G., Dahl, D. W., Gorn, G. J., Weinberg, C. B., Park, J., \& Jiang, Y. (2008). Condom embarrassment: coping and consequences for condom use in three countries. AIDS Care, 20(5), 553-559.

Morales-Campos, D. Y., Markham, C., Peskin, M. F., \& Fernandez, M. E. (2012). Sexual initiation, parent practices, and acculturation in hispanic seventh graders. Journal of School Health, 82(2), 75-81. https://doi.org/10.1111/j.1746-1561.2011.00669.x

Morojele, N. K., Brook, J. S., \& Kachieng'a, M. a. (2006). Perceptions of sexual risk behaviours and substance abuse among adolescents in South Africa: a qualitative investigation. AIDS Care, 18(3), 215-219. https://doi.org/10.1080/09540120500456243

Morrison-Beedy, D., Jones, S. H., Xia, Y., Tu, X., Crean, H. F., \& Carey, M. P. (2012). Reducing Sexual Risk Behavior in Adolescent Girls: Results From a Randomized Controlled Trial. Journal of Adolescent Health, 52, 314321. https://doi.org/10.1016/j.jadohealth.2012.07.005

Morrison, A. B., \& Chein, J. M. (2011). Does working memory training work? The promise and challenges of enhancing cognition by training working memory. Psychonomic Bulletin \& Review, 18(1), 46-60. https://doi.org/10.3758/s13423-010-0034-0

Muchomba, F. M., Chan, C., \& El-Bassel, N. (2015). Importance of Women's Relative Socioeconomic Status within Sexual Relationships in Communication about Safer Sex and HIV/STI Prevention. Journal of Urban Health, 92(3), 559-571. https://doi.org/10.1007/s11524-014-9935-y

Muñoz-Silva, A., Sánchez-García, M., Nunes, C., \& Martins, A. (2 007 ). Gender differences in condom use prediction with Theory of Reasoned Action and Planned Behaviour: the role of self-efficacy and control. AIDS Care, 19(9), 1177-1181.

Murdock, G. P., \& Kluckhohn, R. (1962). Culture and Behavior: Collected Essays of Clyde Kluckhohn. American Sociological Review, 27(5), 721. https://doi.org/10.2307/2089661

Myers, R. (1990). Classical and Modern Regression with Application (2nd ed.). Boston: Duxbury.

Nagel, J. (1994). Constructing Ethnicity: Creating and Recreating Ethnic Identity and Culture. Social Problems, 41(1), 152-176.

Nahmias, S. B., \& Nahmias, D. (2011). Society, sex, and STIs: Human behavior and the evolution of sexually transmitted diseases and their agents. Annals of the New York Academy of Sciences, 1230(1), 59-73. https://doi.org/10.1111/j.1749-6632.2011.06079.x

Napper, L. E., Fisher, D. G., Reynolds, G. L., \& Johnson, M. E. (2010). HIV Risk Behavior Self-Report Reliability at Different Recall Periods. AIDS and Behavior, 14(1), 152-161. https://doi.org/10.1007/s10461-009-9575-5

Neubauer, K., von Auer, M., Murray, E., Petermann, F., Helbig-Lang, S., \& Gerlach, A. L. (2013). Internetdelivered attention modification training as a treatment for social phobia: A randomized controlled trial. Behaviour Research and Therapy, 51(2), 87-97. https://doi.org/10.1016/j.brat.2012.10.006

Newby, K., Wallace, L. M., Dunn, O., \& Brown, K. E. (2012). A survey of English teenagers' sexual experience and preferences for school-based sex education. Sex Education, 12(2), 231-251. https://doi.org/10.1080/14681811.2011.615582

Newman, J. P., Widom, C. S., \& Nathan, S. (1985). Passive Avoidance in Syndromes of Disinhibition. Psychopathy and Extraversion. Journal of Personality and Social Psychology, 48, 1316-1327. https://doi.org/10.1037/0022-3514.48.5.1316

Noar, S. M., Black, H. G., \& Pierce, L. B. (2009). Efficacy of computer technology-based HIV prevention interventions: a meta-analysis. AIDS (London, England), 23(1), 107-115. 
Norman, D. A., \& Shallice, T. (1986). Attention to action: willed and automatic control of behavior. In R. J. Davidson, G. E. Schwartz, \& D. Shapiro (Eds.), Consciousness and self-regulation: Advances in research and theory (Vol. 4, pp. 1-18). Plenum.

Nosek, B. a., Greenwald, a. G., \& Banaji, M. R. (2007). The Implicit Association Test at Age 7: A Methodological and Conceptual Review. Social Psychology and the Unconscious: The Automaticity of Higher Mental Processes, 265-292. https://doi.org/10.1016/j.mrfmmm.2009.01.007

Nunnally, J., \& Bernstein, I. (1994). Psychometric Theory (3rd ed.). New York: McGraw-Hill.

Nyhan, B., Reifler, J., Richey, S., \& Freed, G. L. (2014). Effective Messages in Vaccine Promotion: A Randomized Trial. PEDIATRICS, 133(4), e835-e842. https://doi.org/10.1542/peds.2013-2365

O'Sullivan, L. F. (2015). High texting rates mediate oral sex and intercourse experience in a longitudinal study of high school students. Computers in Human Behavior, 49, 526-531.

O'Sullivan, L. F., \& Allgeier, E. R. (1998). Feigning sexual desire: Consenting to unwanted sexual activity in heterosexual dating relationships. Journal of Sex Research, 35(May), 234-243.

O'Sullivan, L. F., Udell, W., Montrose, V. a., Antoniello, P., \& Hoffman, S. (2010). A cognitive analysis of college students' explanations for engaging in unprotected sexual intercourse. Archives of Sexual Behavior, 39(5), 1121-1131. https://doi.org/10.1007/s10508-009-9493-7

Orji, R., Mandryk, R. L., \& Vassileva, J. (2017). Improving the Efficacy of Games for Change Using Personalization Models. ACM Transactions on Computer-Human Interaction, 24(5), 1-22. https://doi.org/10.1145/3119929

Orland, B., Ram, N., Lang, D., Houser, K., Kling, N., \& Coccia, M. (2014). Saving energy in an office environment: A serious game intervention. Energy and Buildings, 74, 43-52. https://doi.org/10.1016/ j.enbuild.2014.01.036

Ott, M. A., \& Santelli, J. S. (2007). Abstinence and abstinence-only education. Current Opinion in Obstetrics and Gynecology, 19(5), 446-452. https://doi.org/10.1097/GCO.0b013e3282efdc0b

Parong, J., Mayer, R. E., Fiorella, L., MacNamara, A., Homer, B. D., \& Plass, J. L. (2017). Learning executive function skills by playing focused video games. Contemporary Educational Psychology, 51, 141-151. https://doi.org/10.1016/j.cedpsych.2017.07.002

Patel, P., Borkowf, C. B., Brooks, J. T., Lasry, A., Lansky, A., \& Mermin, J. (2014a). Estimating per-act HIV transmission risk. Aids, 28, 1509-1519. https://doi.org/10.1097/QAD.0000000000000298

Patel, P., Borkowf, C. B., Brooks, J. T., Lasry, A., Lansky, A., \& Mermin, J. (2014b). Estimating per-act HIV transmission risk. AIDS, 28(10), 1509-1519. https://doi.org/10.1097/QAD.0000000000000298

Patel, S. H., \& Azzam, P. N. (2005). Characterization of N200 and P300: Selected studies of the Event-Related Potential. International Journal of Medical Sciences, 2(4), 147-154.

Patrick, M. E., \& Maggs, J. L. (2009). Does drinking lead to sex? Daily alcohol-sex behaviors and expectancies among college students. Psych olog y of Ad d ictive Beh a viors: Jou rn a l of th e Society of Psych olog ists in Addictive Behaviors, 23(3), 472-481.

Patton, G. C., Sawyer, S. M., Santelli, J. S., Ross, D. A., Afifi, R., Allen, N. B., ... Viner, R. M. (2016). Our future: a Lancet commission on adolescent health and wellbeing. The Lancet, 387. https://doi.org/10.1016/S01406736(16)00579-1

Patton, J. H., Stanford, M. S., \& Barratt, E. S. (1995). Factor structure of the Barratt impulsiveness scale. Journal of Clinical Psychology, 51(6), 768-774.

Perchet, C., \& García-Larrea, L. (2000). Visuospatial attention and motor reaction in children: an electrophysiological study of the "Posner" paradigm. Psychophysiology, 37(2), 231-241.

Peter, J., \& Valkenburg, P. M. (2016). Adolescents and Pornography: A Review of 20 Years of Research. The Journal of Sex Research, 53(4-5), 509-531. https://doi.org/10.1080/00224499.2016.1143441

Peterman, T. A., Lin, L. S., Newman, D. R., Kamb, M. L., Bolan, G., Zenilman, J., ... Malotte, C. K. (2000). Does measured behavior reflect STD risk?: an analysis of data from a randomized controlled behavioral intervention study. Sexually Transmitted Diseases, 27(8), 446-451.

Peters, G.-J. Y., Ruiter, R. a. C., \& Kok, G. (2012). Threatening communication: a critical re-analysis and a revised meta-analytic test of fear appeal theory. Health Psychology Review, (May 2013), 1-24. https://doi.org/10.1080/17437199.2012.703527 
Pettifor, A., Stoner, M., Pike, C., \& Bekker, L.-G. (2018). Adolescent lives matter. Current Opinion in HIV and AIDS, 13(3), 265-273. https://doi.org/10.1097/COH.0000000000000453

Philpott, A., Knerr, W., \& Boydell, V. (2006). Pleasure and Prevention: When Good Sex Is Safer Sex. Reproductive Health Matters, 14(28), 23-31. https://doi.org/10.1016/S0968-8080(06)28254-5

Piot, P., Abdool Karim, S. S., Hecht, R., Legido-Quigley, H., Buse, K., Stover, J., ... Sidibé, M. (2015). The Lancet Commissions A UNAIDS-Lancet Commission on Defeating AIDS-Advancing Global Health Defeating AIDS-advancing global health. The Lancet, 386, 171-218. https://doi.org/10.1016/S01406736(15)60658-4

Polich, J. (2007). Updating P300: An integrative theory of P3a and P3b. Clinical Neurophysiology, 118(10), 21282148.

Pollak, S. D., \& Tolley-Schell, S. A. (2003). Selective attention to facial emotion in physically abused children. Journal of Abnormal Psychology, 112(3), 323-338.

Portnoy, D. B., Scott-Sheldon, L. A. J., Johnson, B. T., \& Carey, M. P. (2008). Computer-delivered interventions for health promotion and behavioral risk reduction: A meta-analysis of 75 randomized controlled trials, 1988-2007. Preventive Medicine, 47(1), 3-16.

Prabawanti, C., Dijkstra, A., Riono, P., \& Hartana, G. (2014). Preparatory behaviours and condom use during receptive and insertive anal sex among male-to-female transgenders (Waria) in Jakarta, Indonesia. Journal of the International AIDS Society, 17, 1-7.

Prause, N., Steele, V. R., Staley, C., \& Sabatinelli, D. (2015). Late positive potential to explicit sexual images associated with the number of sexual intercourse partners. Social Cognitive and Affective Neuroscience. https://doi.org/10.1093/scan/nsu024

Prensky, M. (2007). Digital Game-based Learning: Practical Ideas for the Application of Digital Game-based Learninh. St. Paul: Paragon House.

Randolph, M. E., Pinkerton, S. D., Bogart, L. M., Cecil, H., \& Abramson, P. R. (2007). Sexual Pleasure and Condom Use. Archives of Sexual Behavior, 36(6), 844-848. https://doi.org/10.1007/s10508-007-9213-0

Rawson, H. A., \& Liamputtong, P. (2010). Culture and sex education: the acquisition of sexual knowledge for a group of Vietnamese Australian young women. Ethnicity \& Health, 15(4), 343-364.

Rehm, J., Shield, K. D., Joharchi, N., \& Shuper, P. A. (2012). Alcohol consumption and the intention to engage in unprotected sex: systematic review and meta-analysis of experimental studies. Addiction, 107(1), 51-59. https://doi.org/10.1111/j.1360-0443.2011.03621.x

Remington, R. W., Folk, C. L., \& McLean, J. P. (2001). Contingent attentional capture or delayed allocation of attention? Perception \& Psychophysics, 63(2), 298-307.

Reyna, V. F., \& Farley, F. (2006). Risk and Rationality in Adolescent Decision Making: Implications for Theory, Practice, and Public Policy. Psychological Science in the Public Interest, 7(1), 1-44. https://doi.org/10. 1111/j.1529-1006.2006.00026.x

Reynolds, B. (2006). A review of delay-discounting research with humans: Relations to drug use and gambling. Behavioural Pharmacology, 17, 6651-6667. https://doi.org/10.1097/FBP.0b013e3280115f99

Rideout, V. J., Roberts, D. F., \& Foehr, U. G. (2005). Generation M: Media in the lives of 8-18 year olds (Kaiser Family Foundation Studies). Victoria. Washington: Kaiser Family Foundation.

Rogow, D., Haberland, N., Del Valle, A., Lee, N., Osakue, G., Sa, Z., \& Skaer, M. (2013). Integrating gender and rights into sexuality education: Field reports on using It's all One. Reproductive Health Matters, 21(41), 154-166. https://doi.org/10.1016/S0968-8080(13)41699-3

Romer, D. (2010). Adolescent risk taking, impulsivity, and brain development: Implications for prevention. Developmental Psychobiology, 52(3), 263-276. https://doi.org/10.1002/dev.20442

Rooke, S. E., Hine, D. W., \& Thorsteinsson, E. B. (2008). Implicit cognition and substance use: A meta-analysis. Addictive Behaviors, 33(10), 1314-1328. https://doi.org/10.1016/j.addbeh.2008.06.009

Rostosky, S. S., Wilcox, B. L., Wright, M. L. C., \& Randall, B. A. (2004). The Impact of Religiosity on Adolescent Sexual Behavior: Journal of Adolescent Research, 19(6), 677-697. https://doi.org/10.1177/074355 8403260019

Rothermund, K., \& Wentura, D. (2001). Rothermund \& Wentura. Zeitschrift Für Experimentelle Psychologie. 
Rozin, P., \& Millman, L. (1987). Family environment, not heredity, accounts for family resemblances in food preferences and attitudes: A twin study. Appetite, 8, 125-134. https://doi.org/10.1016/S01956663(87)80005-3

Rydell, R. J., \& McConnell, A. R. (2006). Understanding implicit and explicit attitude change: A systems of reasoning analysis. Journal of Personality and Social Psychology, 91(6), 995-1008. https://doi.org/10. 1037/0022-3514.91.6.995

Sabini, J., Garvey, B., \& Hall, A. L. (2016). Shame and Embarrassment Revisited. Personality and Social Psychology Bulletin, 27(1), 104-117.

Sales, J. M., Smearman, E. L., Swartzendruber, A., Brown, J. L., Brody, G., \& DiClemente, R. J. (2014). Socioeconomic-Related Risk and Sexually Transmitted Infection Among African-American Adolescent Females. Journal of Adolescent Health, 55(5), 698-704. https://doi.org/10.1016/j.jadohealth.2014.05.005

Sanders, S. A., Reece, M., Herbenick, D., Schick, V., Dodge, B., \& Fortenberry, J. D. (2010). Condom Use During Most Recent Vaginal Intercourse Event Among a Probability Sample of Adults in the United States. The Journal of Sexual Medicine, 7, 362-373. https://doi.org/10.1111/j.1743-6109.2010.02011.x

Sardi, L., Idri, A., \& Fernández-Alemán, J. L. (2 $\begin{array}{llll}2 & 0 & 1 & 7\end{array}$ ). A systematic review of gamificationtiteelth. Journal of Biomedical Informatics, 71, 31-48. https://doi.org/10.1016/j.jbi.2017.05.011

Sawyer, S. M., Afifi, R. A., Bearinger, L. H., Blakemore, S. J., Dick, B., Ezeh, A. C., \& Patton, G. C. (2012). Adolescence: A foundation for future health. The Lancet, 379(9826), 1630-1640.

Schaalma, H. P., Abraham, C., Gillmore, M. R., \& Kok, G. (2004). Sex education as health promotion: what does it take? Archives of Sexual Behavior, 33(3), 259-269. https://doi.org/10.1023/B:ASEB.0000026625. $65171.1 \mathrm{~d}$

Schachar, R., Logan, G. D., Robaey, P., Chen, S., Ickowicz, A., \& Barr, C. (2007). Restraint and cancellation: Multiple inhibition deficits in attention deficit hyperactivity disorder. Journal of Abnormal Child Psychology, 35, 229-238. https://doi.org/10.1007/s10802-006-9075-2

Schalet, A. T. (2011). Beyond Abstinence and Risk: A New Paradigm for Adolescent Sexual Health. Women's Health Issues, 21(3), S5-S7. https://doi.org/10.1016/j.whi.2011.01.007

Schmitt, D. P. (2005). Sociosexuality from Argentina to Zimbabwe: a 48-nation study of sex, culture, and strategies of human mating. The Behavioral and Brain Sciences, 28(2), 247-275; discussion 275-311.

Schoemaker, P. J. H. (1990). Are Risk-Attitudes Related Across Domains and Response Modes? Management Science, 36(12), 1451-1463. https://doi.org/10.1287/mnsc.36.12.1451

Schofield, H.-L. T., Bierman, K. L., Heinrichs, B., \& Nix, R. L. (2008). Predicting early sexual activity with behavior problems exhibited at school entry and in early adolescence. Journal of Abnormal Child Psychology, 36(8), 1175-1188. https://doi.org/10.1007/s10802-008-9252-6

Schupp, H. T., Cuthbert, B. N., Bradley, M. M., Cacioppo, J. T., Tiffany, I., \& Lang, P. J. (2000). Affective picture processing: The late positive potential is modulated by motivational relevance. Psychophysiology. https://doi.org/10.1017/S0048577200001530

Schupp, H. T., Junghöfer, M., Weike, A. I., \& Hamm, A. O. (2004). The selective processing of briefly presented affective pictures: An ERP analysis. Psychophysiology. https://doi.org/10.1111/j.1469-8986.2004.00174.x

Schutte, L., Meertens, R. M., Mevissen, F. E. F., Schaalma, H., Meijer, S., \& Kok, G. (2014). Long Live Love. The implementation of a school-based sex-education program in the Netherlands. Health Education Research, 29(4), 583-597. https://doi.org/10.1093/her/cyu021

Seidman, S. N., Mosher, W. D., \& Aral, S. O. (1994). Predictors of high-risk behavior in unmarried American women: Adolescent environment as a risk factor. Journal of Adolescent Health, 15, 126-132.

Sennwald, V., Pool, E., Brosch, T., Delplanque, S., Bianchi-Demicheli, F., \& Sander, D. (2016). Emotional attention for erotic stimuli: Cognitive and brain mechanisms. Journal of Comparative Neurology, 524(8), 1668-1675. https://doi.org/10.1002/cne.23859

Ševčíková, A., Blinka, L., \& Daneback, K. $\left(\begin{array}{llll}2 & 0 & 1 & 8\end{array}\right)$. Sexting as a predictor of sexual behavior in a sample of Czech adolescents. European Journal of Developmental Psychology, 15(4), 426-437. https://doi.org/10.1080/ 17405629.2017.1295842

Shad, M. U., Bidesi, A. S., Chen, L. A., Thomas, B. P., Ernst, M., \& Rao, U. (2011). Neurobiology of decision-making in adolescents. Behavioural Brain Research, 217(1), 67-76. 
Shannon, C. L., \& Klausner, J. D. (2018). The growing epidemic of sexually transmitted infections in adolescents. Current Opinion in Pediatrics, 30(1), 137-143. https://doi.org/10.1097/MOP.00000 00000000578

Sharma, S., Arain, Mathur, Rais, Nel, Sandhu, ... Johal. (2013). Maturation of the adolescent brain. Neuropsychiatric Disease and Treatment, 9, 449-460. https://doi.org/10.2147/NDT.S39776

Sheeran, P. (2002a). Intention-behavior relations: A conceptual and empirical review. European Review of Social Psychology, 12(October), 1-36.

Sheeran, P. (2002b). Intention-Behavior Relations: A Conceptual and Empirical Review. European Review of Social Psychology, 12(1), 1-36.

Sheeran, P., \& Orbell, S. (1998). Do intentions predict condom use? Meta-analysis and examination of six moderator variables. The British Journal of Social Psychology / the British Psychological Society, 37 ( Pt 2), 231-250.

Sheeran, P., Webb, T. L., \& Gollwitzer, P. M. (2005). The interplay between goal intentions and implementation intentions. Personality \& Social Psychology Bulletin, 31, 87-98.

Shepherd, L. M., Sly, K. F., \& Girard, J. M. (2017). Comparison of comprehensive and abstinence-only sexuality education in young African American adolescents. Journal of Adolescence, 61, 50-63. https://doi.org/10.1016/j.adolescence.2017.09.006

Sieverding, J. A., Adler, N., Witt, S., \& Ellen, J. (2005). The influence of parental monitoring on adolescent sexual initiation. Archives of Pediatrics \& Adolescent Medicine, 159(8), 724-729.

Silvério Marques, S., Goldfarb, E. S., Deardorff, J., \& Constantine, N. A. (2017). Perspectives on Conceptualizing Developmentally Appropriate Sexuality Education. American Journal of Sexuality Education, 12(1), 35-54. https://doi.org/10.1080/15546128.2016.1266442

Singer, M. (2010). AIDS, Sex and Culture: Global Politics and Survival in Southern Africa. The Australian Journal of Anthropology, 21(3), 395-396.

Sinha, J. W., Cnaan, R. A., \& Gelles, R. J. (2007). Adolescent risk behaviors and religion: Findings from a national study. Journal of Adolescence, 30(2), 231-249. https://doi.org/10.1016/j.adolescence. 2006.02.005

Skakoon-Sparling, S., \& Cramer, K. M. (2016). The impact of sexual arousal on elements of sexual decision making: Sexual self-restraint, motivational state, and self-control. The Canadian Journal of Human Sexuality, 25(2), 119-125. https://doi.org/10.3138/cjhs.252-A1

Skakoon-Sparling, S., Cramer, K. M., \& Shuper, P. A. (2015). The Impact of Sexual Arousal on Sexual Risk-Taking and Decision-Making in Men and Women. Archives of Sexual Behavior, 45, 33-42. https://doi.org/10.1007/s10508-015-0589-y

Skakoon-Sparling, S., Cramer, K. M., \& Shuper, P. A. (2016). The Impact of Sexual Arousal on Sexual Risk-Taking and Decision-Making in Men and Women. Archives of Sexual Behavior, 45(1), $33-42$. https://doi.org/10.1007/s10508-015-0589-y

Smerecnik, C., Schaalma, H., Kok, G., Meijer, S., \& Poelman, J. (2010). An exploratory study of Muslim adolescents' views on sexuality: Implications for sex education and prevention. BMC Public Health, 10, 533.

Smith, A. M. A., \& de Visser, R. O. (2004). Which intention? Whose intention? Condom use and theories of individual decision making. Psychology, Health \& Medicine, 9(2), 193-204. https://doi.org/10.1080/ 13548500410001670717

Smith, A. R., Chein, J., \& Steinberg, L. (2014). Peers increase adolescent risk taking even when the probabilities of negative outcomes are known. Developmental Psychology, 50(5). https://doi.org/10.1037/a0035696

Snipes, D. J., \& Benotsch, E. G. (2013). High-risk cocktails and high-risk sex: Examining the relation between alcohol mixed with energy drink consumption, sexual behavior, and drug use in college students. Addictive Behaviors, 38(1), 1418-1423.

Somerville, L. H. (2013). The Teenage Brain: Sensitivity to Social Evaluation. Current Directions in Psychological Science, 22(2), 121-127. https://doi.org/10.1177/0963721413476512

Somerville, L. H., Jones, R. M., \& Casey, B. J. (2010). A time of change: Behavioral and neural correlates of adolescent sensitivity to appetitive and aversive environmental cues. Brain and Cognition, 72(1), 124-133. https://doi.org/10.1016/j.bandc.2009.07.003 
Spanlang, B., Normand, J.-M., Giannopoulos, E., \& Slater, M. (2010). A first person avatar system with haptic feedback. In Proceedings of the 17th ACM Symposium on Virtual Reality Software and Technology - VRST '10 (pp. 47-5 0 ). ACM Press. Retrieved from http://portal.acm.org/citation.cfm?doid=1 88898860.18898 Spear, L. P. (2009). The Behavioral Neuroscience of Adolescence. New York: Norton.

Stacy, A. W., Ames, S. L., Ullman, J. B., Zogg, J. B., \& Leigh, B. C. (2006). Spontaneous cognition and HIV risk behavior. Psych olog y of Ad d ictive Beh a viors: JouthenSacilety of Psychologists in Addictive Behaviors, 20(2), 196-206.

Stacy, A. W., \& Wiers, R. W. (2010). Implicit Cognition and Addiction: A Tool for Explaining Paradoxical Behavior. Annual Review of Clinical Psychology, 6(1), 551-575. https://doi.org/10.1146/annurev. clinpsy.121208.131444

Stanger-Hall, K. F., \& Hall, D. W. (2011). Abstinence-only education and teen pregnancy rates: Why we need comprehensive sex education in the U.S. PLOS ONE, 6(10).

Starks, K. (2014). Cognitive behavioral game design: A unified model for designing serious games. Frontiers in Psychology, 5(FEB), 1-10. https://doi.org/10.3389/fpsyg.2014.00028

Statistisches Bundesamt. (2015). Bevölkerung und Erwerbstätigkeit. Wiesbaden. Retrieved from https://www.destatis.de/DE/Publikationen/Thematisch/Bevoelkerung/MigrationIntegration/Migrationsh

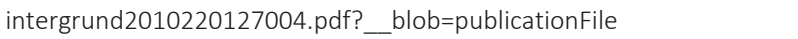

Steinberg, L. (2008). A social neuroscience perspective on adolescent risk-taking. Developmental Review, 28(1), 78-106. https://doi.org/10.1016/j.dr.2007.08.002

Steinberg, L. (2010). A dual systems model of adolescent risk-taking. Developmental Psychobiology, 52(3), 216224.

Steinberg, L. (2011). Adolescent Risk Taking: A Social Neuroscience Perspective. In E. Amsel \& J. Smetana (Eds.), Adolescent vulnerabilities and opportunities: Developmental and constructivist perspectives (pp. 41-64). Cambridge: Cambridge University Press.

Steinberg, L., \& Monahan, K. C. (2007). Age differences in resistance to peer influence. Developmental Psychology, 43(6), 1531-1543. https://doi.org/10.1037/0012-1649.43.6.1531

Sterrett, E. M., Jones, D. J., \& Kincaid, C. (2009). Psychosocial adjustment of low-income African American youth from single mother homes: the role of the youth-coparent relationship. Journal of Clinical Child and Adolescent Psychology, 38(3), 427-438.

Strack, F., \& Deutsch, R. (2004). Reflective and impulsive determinants of social behavior. Personality and Social

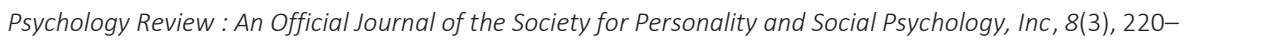
247.

Strang, N. M., Chein, J. M., \& Steinberg, L. (2013). The value of the dual systems model of adolescent risk-taking. Frontiers in Human Neuroscience, 7(May), 223. https://doi.org/10.3389/fnhum.2013.00223

Sturman, D. A., \& Moghaddam, B. (2012). Striatum processes reward differently in adolescents versus adults. Proceedings of the National Academy of Sciences, 109(5), 1719-1724.

Suh, K.-S., Kim, H., \& Suh, E. K. (2011). What if your avatar looks like you? Dual-Congruity perspectives for avatar use. MIS Quarterly, 35(3), 711-A4.

Sweldens, S., Van Osselaer, S. M. J., \& Janiszewski, C. (2010). Evaluative Conditioning Procedures and the Resilience of Conditioned Brand Attitudes. Journal of Consumer Research, 37(3), 473-489. https://doi.org/10.1086/653656

Taylor, B. M. (1995). Gender-power relations and safer sex negotiation. Journal of Advanced Nursing, 22(4), 687-693.

Terris-Prestholt, F., \& Windmeijer, F. (2016). How to sell a condom? The impact of demand creation tools on male and female condom sales in resource limited settings. Journal of Health Economics, 48, 107-120. https://doi.org/10.1016/j.jhealeco.2016.04.001

Teva, I., Bermudez, M. P., Ramiro, M. T., \& Ramiro-Sanchez, T. (2013). Analysis of sexual behavior in adolescents. Current HIV Research, 11(7), 512-519. https://doi.org/10.2174/1570162X12666140129095811

Thush, C., Wiers, R. W., Ames, S. L., Grenard, J. L., Sussman, S., \& Stacy, A. W. (2008). Interactions between implicit and explicit cognition and working memory capacity in the prediction of alcohol use in at-risk adolescents. Drug and Alcohol Dependence, 1(94), 1-3. https://doi.org/10.1016/j.drugalcdep.2007.10.019 
Tiblom Ehrsson, Y., Stenhammar, C., Rosenblad, A., Åkerud, H., Larsson, M., \& Tydén, T. (2016). Self-reported sexually transmitted infections among female university students. Upsala Journal of Medical Sciences, 121(1), 45-49. https://doi.org/10.3109/03009734.2015.1093568

Trenholm, C., Devaney, B., Fortson, K., Clark, M., Quay, L., \& Wheeler, J. (2008). Impacts of abstinence education on teen sexual activity, risk of pregnancy, and risk of sexually transmitted diseases. Journal of Policy Analysis and Management, 27(2), 255-276. https://doi.org/10.1002/pam.20324

Trepte, S., \& Reinecke, L. (2010). Avatar creation and video game enjoyment. Journal of Media Psychology, 22(4), 171-184.

Tschann, J. M., Flores, E., De Groat, C. L., Deardorff, J., \& Wibbelsman, C. J. (2010). Condom negotiation strategies and actual condom use among latino youth. Journal of Adolescent Health, 47(3), 254-262. https://doi.org/10.1016/j.jadohealth.2010.01.018

Turchik, J. A., \& Gidycz, C. A. (2012). Exploring the Intention-Behavior Relationship in the Prediction of Sexual Risk Behaviors: Can It Be Strengthened? Journal of Sex Research, 49(1), 50-60.

Uccella, I., Petrelli, A., Vescio, M. F., De Carolis, S., Fazioli, C., Pezzotti, P., \& Rezza, G. (2017). HIV rapid testing in the framework of an STI prevention project on a cohort of vulnerable Italians and immigrants. AIDS Care, 29(8), 996-1002. https://doi.org/10.1080/09540121.2017.1281876

Unaids. (2013). Global report: UNAIDS report on the global AIDS epidemic 2013. Unaids. https://doi.org/JC2502/1/E

UNAIDS. (2011). Fact sheet 2011. Geneva. Retrieved from http://files.unaids.org/en/media/unaids/ contentassets/documents/factsheet/2011/20111121_FS_WAD2011_global_en.pdf

UNAIDS. (2014). Fact sheet 2014. Geneva. Retrieved from http://files.unaids.org/en/media/unaids/ contentassets/documents/factsheet/2014/20140716_FactSheet_en.pdf

UNAIDS. (2016). 2016 United Nations Political Declaration on HIV and AIDS: On the Fast-Track to Accelerate the Fight against HIV and to End the AIDS Epidemic by 2030. Geneva. Retrieved from http://www.unaids.org/en/resources/documents/2016/2016-political-declaration-HIV-AIDS

UNAIDS. (2017). UNAIDS data 2017. Geneva. Retrieved from http://www.unaids.org/en/resources/ documents/2017/2017_data_book

UNAIDS. (2018). Global AIDS Monitoring 2018. Indicators for monitoring the 2016 United Nations Political Declaration on Ending AIDS. Geneva. Retrieved from http://www.unaids.org/sites/default/files/ media_asset/2017-Global-AIDS-Monitoring_en.pdf

UNICEF. (2017). Children and AIDS: Statistical update. Retrieved from https://data.unicef.org/wpcontent/uploads/2017/11/HIVAIDS-Statistical-Update-2017.pdf

United Nations. (2015). World Population Prospects. The 2015 Revision. New York. Retrieved from https://esa.un.org/unpd/wpp/publications/files/key_findings_wpp_2015.pdf

Van Damme, S., \& Crombez, G. (2009). Measuring attentional bias to threat in children and adolescents: A matter of speed? Journal of Behavior Therapy and Experimental Psychiatry, 40(2), 344-351.

Van Empelen, P., \& Kok, G. (2006). Condom use in steady and casual sexual relationships: Planning, preparation and willingness to take risks among adolescents. Psychology and Health, 21(2), 165-181. https://doi.org/10.1080/14768320500229898

Van Empelen, P., \& Kok, G. (2008). Action-specific cognitions of planned and preparatory behaviors of condom use among Dutch adolescents. Archives of Sexual Behavior, 37(4), 626-640. https://doi.org/10.1007/ s10508-007-9286-9

Van Ryzin, M. J., Johnson, A. B., Leve, L. D., \& Kim, H. K. (2011). The number of sexual partners and health-risking sexual behavior: Prediction from high school entry to high school exit. Archives of Sexual Behavior, 40(5), 939-949. https://doi.org/10.1007/s10508-010-9649-5

Van Veen, V., \& Carter, C. S. (2006). Conflict and cognitive control in the brain. Current Directions in Psychological Science. https://doi.org/10.1111/j.1467-8721.2006.00443.x

Vannier, S. a, \& O'Sullivan, L. F. (2010). Sex without desire: characteristics of occasions of sexual compliance in young adults' committed relationships. Journal of Sex Research, 47(5), 429-439. 
Vardi, Y., Volos, M., Sprecher, E., Granovsky, Y., Gruenwald, I., \& Yarnitsky, D. (2006). A P300 Event Related Potential Technique for Assessment of Sexually Oriented Interest. Journal of Urology. https://doi.org/ 10.1016/j.juro.2006.07.134

Varghese, B., Maher, J. E., Peterman, T. a, Branson, B. M., \& Steketee, R. W. (2002). Reducing the risk of sexual HIV transmission: quantifying the per-act risk for HIV on the basis of choice of partner, sex act, and condom use. Sexually Transmitted Diseases, 29(1), 38-43.

Verbeken, S., Braet, C., Naets, T., Houben, K., \& Boendermaker, W. (2018). Computer training of attention and inhibition for youngsters with obesity: A pilot study. Appetite, 123, 439-447. https://doi.org/10.1016/ j.appet.2017.12.029

Verbruggen, F., \& Logan, G. (2008). Response inhibition in the stop-signal pardigm. Trends in Cognitive Sciences. https://doi.org/10.1016/j.tics.2008.07.005.Response

Verbruggen, F., \& Logan, G. D. (2009). Models of response inhibition in the stop-signal and stop-change paradigms. Neuroscience and Biobehavioral Reviews. https://doi.org/10.1016/j.neubiorev.2008.08.014

Vidal, J., Mills, T., Pang, E. W., \& Taylor, M. J. (2012). Response inhibition in adults and teenagers: Spatiotemporal differences in the prefrontal cortex. Brain and Cognition. https://doi.org/10.1016/ j.bandc.2011.12.011

Vogeley, K., \& Fink, G. (2003). Neural correlates of the first-person-perspective. Trends in Cognitive Sciences, $7(1), 38-42$.

Vogeley, K., May, M., Ritzl, A., Falkai, P., Zilles, K., \& Fink, G. R. (2004). Neural correlates of first-person perspective as one constituent of human self-consciousness. Journal of Cognitive Neuroscience, 16(5), 817-827.

von Bastian, C. C., Langer, N., Jäncke, L., \& Oberauer, K. (2013). Effects of working memory training in young and old adults. Memory \& Cognition, 41(4), 611-624. https://doi.org/10.3758/s13421-012-0280-7

Von Bastian, C. C., \& Oberauer, K. (2013). Distinct transfer effects of training different facets of working memory capacity. Journal of Memory and Language, 69(1), 36-58. https://doi.org/10.1016/j.jml. 2013.02.002

Wardaszko, M., \& Podgórski, B. (2017). Mobile Learning Game Effectiveness in Cognitive Learning by Adults: A Comparative Study. Simulation \& Gaming, 48(4), 435-454. https://doi.org/10.1177/1046878117704350

Webb, T. L., \& Sheeran, P. (2006). Does changing behavioral intentions engender behavior change? A metaanalysis of the experimental evidence. Psychological Bulletin, 132(2), 249-268.

Weber, E. U., Blais, A.-R., \& Betz, N. E. (2002). A domain-specific risk-attitude scale: Measuring risk perceptions and risk behaviors. Journal of Behavioral Decision Making, 15(August), 263-290. https://doi.org/10.1002/bdm.414

Weber, E. U., \& Hsee, C. (1998). Cross-Cultural Differences in Risk Perception, but Cross-Cultural Similarities in Attitudes Towards Perceived Risk. Management Science.

Weber, E. U., \& Milliman, R. a. (1997). Perceived Risk Attitudes: Relating Risk Perception to Risky Choice. Management Science, 43(2), 123-144. https://doi.org/10.1287/mnsc.43.2.123

Weeks, M. R., Coman, E., Hilario, H., Li, J., \& Abbott, M. (2013). Initial and Sustained Female Condom Use Among Low-Income Urban U.S. Women. Journal of Women's Health, 22(1), 26-36. https://doi.org/10.1089/jwh.2011.3430

Weigard, A., Chein, J., Albert, D., Smith, A., \& Steinberg, L. (2014). Effects of anonymous peer observation on adolescents' preference for immediate rewards. Developmental Science, 17(1), 71-78.

Weller, S., \& Davis, K. (2002). Condom effectiveness in reducing heterosexual HIV transmission. Cochrane Database of Systematic Reviews (Online), (1), CD003255.

Wetherill, R. R., Neal, D. J., \& Fromme, K. (2010). Parents, peers, and sexual values influence sexual behavior during the transition to college. Archives of Sexual Behavior, 39(3), 682-694. https://doi.org/10.1007/ s10508-009-9476-8

White, H. R., Fleming, C. B., Catalano, R. F., \& Bailey, J. A. (2009). Prospective associations among alcohol userelated sexual enhancement expectancies, sex after alcohol use, and casual sex. Psychology of Addictive

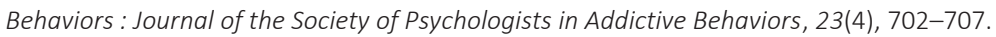

Wickrama, T., Merten, M. J., \& Wickrama, K. A. S. (2012). Early Socioeconomic Disadvantage and Young Adult Sexual Health. American Journal of Health Behavior, 36(6), 834-848. https://doi.org/10.5993/AJHB. 36.6.10 
Widman, L., Choukas-Bradley, S., Noar, S. M., Nesi, J., \& Garrett, K. (2016). Parent-Adolescent Sexual Communication and Adolescent Safer Sex Behavior. JAMA Pediatrics, 170(1), 52. https://doi.org/ 10.1001/jamapediatrics.2015.2731

Widman, L., Noar, S. M., Choukas-Bradley, S., \& Francis, D. B. (2014). Adolescent sexual health communication and condom use: A meta-analysis. Health Psychology, 33(10), 1113-1124. https://doi.org/10.1037/ hea0000112

Wiers, R. W. (2018). Cognitive Training in Addiction: Does It Have Clinical Potential? Biological Psychiatry: Cognitive Neuroscience and Neuroimaging, 3(2), 101-102. https://doi.org/10.1016/J.BPSC.2017.12.008

Wiers, R. W., Beckers, L., Houben, K., \& Hofmann, W. (2009). A short fuse after alcohol: Implicit power associations predict aggressiveness after alcohol consumption in young heavy drinkers with limited executive control. Pharmacology Biochemistry and Behavior, 93(3), 300-305.

Wiers, R. W., Boelema, S. R., Nikolaou, K., \& Gladwin, T. E. (2015). On the Development of Implicit and Control Processes in Relation to Substance Use in Adolescence. Current Addiction Reports, 2(2), 141-155. https://doi.org/10.1007/s40429-015-0053-z

Wiers, R. W., \& Stacy, A. W. (2006). Implicit cognition and addiction. Current Directions in Psychological Science, 15(6), 292-296.

Wiers, W., Jong, P. De, \& Alan, W. (2010). Why Common Sense Goes Out the Window. Handbook of Implicit Social Cognition: Measurement, Theory, and Applications, 463-488. https://doi.org/10.1177/0146 167296228001

Witte, S. S., MacPhee, C., Ginsburg, N., \& Deshmukh, N. (2017). Medicaid Reimbursement for the Female Condom. American Journal of Public Health, 107(10), 1633-1635. https://doi.org/10.2105/AJPH.2017. 303936

Wolfers, M., de Zwart, O., \& Kok, G. (2011). Adolescents in The Netherlands underestimate risk for sexually transmitted infections and deny the need for sexually transmitted infection testing. AIDS Patient Care and STDs, 25(5), 311-319. https://doi.org/10.1089/apc.2010.0186

World Bank. (2018). GDP per capita (current US\$). Retrieved June 14, 2018, from https://data.worldbank.org/ indicator/NY.GDP.PCAP.CD

World Health Organization. (2001). The second decade: improving adolescent health and development. Geneva.

World Health Organization. (2010). Developing sexual health programmes. A framework for action. Geneva.

World Health Organization. (2012). Global incidence and prevalence of selected curable sexually transmitted infections - 2008. Geneva.

World Health Organization. (2013). Report on global sexually transmitted infection surveillance 2013. Geneva.

World Health Organization. (2014). Global update on the health sector response to HIV - 2014. Geneva.

World Health Organization. (2017). Global Accelerated Action for the Health of Adolescents (AA-HA!) Guidance to Support Country Implementation. Retrieved from http://apps.who.int/iris/bitstream/handle/10665/ 255415/9789241512343-eng.pdf;jsessionid=8 O B2 0 BDC4 A4 A3 E8 79304 D8 6 BED9 D3 3 FF?sequence=1

Wouters, P., van Nimwegen, C., van Oostendorp, H., \& van der Spek, E. D. (2013). A meta-analysis of the cognitive and motivational effects of serious games. Journal of Educational Psychology, 105(2), 249-265. https://doi.org/10.1037/a0031311

Wu, Y., Burns, J. J., Stanton, B. F., Li, X., Harris, C. V., Galbraith, J., \& Wei, L. (2005). Influence of prior sexual risk experience on response to intervention targeting multiple risk behaviors among adolescents. Journal of Adolescent Health, 36(1), 56-63. https://doi.org/10.1016/j.jadohealth.2003.09.024

Wulfert, E., \& Wan, C. K. (1993). Condom use: a self-efficacy model. Health Psychology, 12(5), 346-353.

Xiao, Z. (2008). Sensation seeking and impulsivity: the direct and indirect effects on adolescent marijuana use. Journal of Substance Use, 13(6), 415-433. https://doi.org/10.1080/14659890802242437

Xu, Q., Bekteshi, V., \& Tran, T. (2010). Family, School, Country of Birth and Adolescents' Psychological WellBeing. Journal of Immigrant \& Refugee Studies, 8(1), 91-110.

Yang, H., Carmon, Z., Kahn, B., Malani, A., Schwartz, J., Volpp, K., \& Wansink, B. (2012). The Hot-Cold Decision Triangle: A framework for healthier choices. Marketing Letters, 23(2), 457-472.

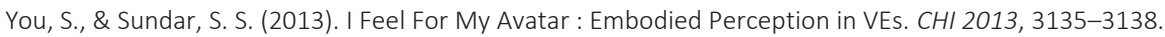


Young, M. a, \& Vazsonyi, A. T. (2011). Parents, peers, and risky sexual behaviors in rural African American adolescents. The Journal of Genetic Psychology, 172(1), 84-93. https://doi.org/10.1080/00221325.2010. 506373

Zimmer-Gembeck, M. J., \& Helfand, M. (2008). Ten years of longitudinal research on U.S. adolescent sexual behavior: Developmental correlates of sexual intercourse, and the importance of age, gender and ethnic background. Developmental Review, 28(2), 153-224.

Zuckerman, M. (1971). Dimensions of sensation seeking. Journal of Consulting and Clinical Psychology, 36(1), 45-52. https://doi.org/10.1037/h0030478 


\section{Appendix}

\section{Reasoned Action Approach items adapted from Fishbein and Ajzen, 2010}

All items were measured using 7-point Likert scales ranging from [1] "unlikely" or "unpleasant/bad" or "disagree" or "false" to [7] "likely" or "pleasant/good" or "agree" or "true".

Intention related to condom use

- I intend to always use condoms when I have sex with a sex partner for the first time.

- I expect that I will always use condoms when I have sex with a sex partner for the first time.

Attitude toward condom use

- Me always using condoms when having sex with a sex partner for the first time would be...

- Me always using condoms when having sex with a sex partner for the first time would be...

Subjective norms related to condom use

- Most people who are important to me approve of me always using condoms when I have sex with a sex partner for the first time.

- Most people like me always use condoms when they have sex with a sex partner for the first time. 
Perceived behavioral control related to condom use

- I am confident that, if I want to, I can always use condoms when I have sex with a sex partner for the first time.

- My use of condoms when I have sex with a sex partner for the first time is up to me.

Intentions related to asking about sexual health status

- I intend to always ask a sex partner about his/her sexual health status before I have sex with him/her for the first time.

- I expect that I will always ask a sex partner about his/her sexual health status before I have sex with him/her for the first time.

Attitude towards asking about sexual health status

- Me always asking my sex partner about his/her sexual health status before I have sex with him/her for the first time would be...

- Me always asking my sex partner about his/her sexual health status before I have sex with him/her for the first time would be...

Subjective norms related to asking about health status

- Most people who are important to me approve of me always asking my sex partner about his/her sexual health status before I have sex with him/her for the first time.

- Most people like me always ask their sex partner about his/her sexual health status before they have sex with him/her for the first time.

Perceived behavioral control related to asking about health status

- I am confident that, if I want to, I can always ask my sex partner about his/her sexual health status before I have sex with him/her for the first time.

- Me asking my sex partner about his/her sexual health status before I have sex with him/her for the first time is up to me.

Intention related to sex practices

- I intend to always practice less risky sex acts when I have sex with a sex partner for the first time.

- I expect that I will always practice less risky sex acts when I have sex with a sex partner for the first time. 
Attitude towards sex practices

- Me always practicing less risky sex acts when having sex with a sex partner for the first time would be...

- Me always practicing less risky sex acts when having sex with a sex partner for the first time would be...

Subjective norms related to sex practices

- Most people who are important to me approve of me always practicing less risky sex acts when I have sex with a sex partner for the first time.

- Most people like me always practice less risky sex acts when they have sex with a sex partner for the first time.

Perceived behavioral control related to sex practices

- I am confident that, if I want to, I can always practice less risky sex acts when I have sex with a sex partner for the first time.

- Me practicing less risky sex acts when I have sex with a sex partner for the first time is up to me. 

Summary 

The present dissertation tested a novel, serious game approach to increase safer-sex behavior in young people while broadening the focus on barrier protection by adding the following two behaviors: asking about a new sex partner's sexual health and the refraining from risky sexual practices, such as penetrative anal sex, with a new sex partner.

In the first chapter, we looked for variables that predicted the engagement of young people in the three protective safer-sex behaviors. We found that young people's intentions to engage in these behaviors were mainly explained by concepts from Fishbein and Ajzen's (2010) Reasoned Action Approach, but that distal variables, e.g., the number of instances of unprotected intercourse during the last year, also added significant predictive power to the intention to perform these behaviors. Qualitative data, reported in chapter two, showed that all three protective behaviors were identified by young people as being protective against the contraction of sexually transmitted infections. However, condom use, although being a rather popular means of protection, was often perceived as a pleasure-reducing "necessary evil" and the other two protective measures were consistently least popular among young people. The idea of engaging in less risky sex acts was repeatedly associated with boredom and rejected on the grounds of reduced sexual pleasure while asking a new sex partner about her or his sexual health status was often perceived as embarrassing and unpleasant, leading to widespread rejection mostly within subgroups reporting low intentions to perform all three protective behaviors. Interestingly, as reported in chapter four, the rejection of asking a new sex partner about her or his current sexual health status was not observed when participants were sexually aroused, reflected in significantly more positive implicit and explicit attitudes towards this behavior as compared to being in a non-aroused state. This suggests that behavioral disinhibition due to sexual arousal is not per se a threat to safer-sex behavior but can also support protective behavior that is perceived as embarrassing in a non-aroused state. That embarrassment can also hamper the translation of condom use intentions into behavior was reported in chapter five. A study using electroencephalography (EEG) showed that a high level of intention alone might be insufficient to predict condom use behavior if its implementation is associated with feelings of embarrassment.

To test if a combination of gamified behavior-change techniques - namely evaluative conditioning, selective response inhibition training, and working memory training - could significantly increase the intentions to perform the three protective behaviors of interest, we developed a serious game with young people aged between 18 and 24 years as target population. Chapter three reports the preferences of young people regarding content and design of such a game during focus group interviews. Participants preferred a realworld design with highly customizable avatars and a game that contained information modules to explain various safer-sex approaches and the use of different barrier protection methods. Further, young people emphasized the importance of including an alcohol intoxicated avatar to increase awareness about the loss of control that can occur while in an intoxicated state. 
The final game aimed at strengthening implicit and explicit determinants of the three safer-sex behaviors of interest. We obtained mixed results that are reported in chapter six. In fact, only when the evaluative conditioning, working memory training, and selective response inhibition components were active during gaming, some explicit determinants were strengthened while others, especially intentions, were weakened after three or five gaming sessions. We suggest that five gaming sessions were not enough to achieve a robust attitude change and that targeting three behaviors in one game might have been simply too much. It seems that, also in a virtual environment, it could be a case of less is more. 
Samenvatting 

In dit proefschrift werd een innovatieve serious game om veilig seksueel gedrag onder jongeren te bevorderen, ontwikkeld en getest. De aandacht richtte zich daarbij niet alleen op het bevorderen van condoomgebruik om seksueel overdraagbare aandoeningen te voorkomen, maar ook op twee additionele veilige gedragingen, namelijk bij een nieuwe partner informeren naar haar of zijn seksuele verleden en de risico's die daarbij mogelijk zijn gelopen en het vermijden van risicovolle seksuele praktijken, zoals het hebben van anale seks met een nieuwe partner.

Het eerste hoofdstuk beschrijft een kwantitatieve studie naar de variabelen die bepalen of jongeren wel of niet bereid zijn tot het uitvoeren van de drie beschermende seksuele gedragingen. Er werd gevonden dat de intentie van jongeren om deze gedragingen uit te voeren voornamelijk werd bepaald door theoretische concepten afkomstig uit de Reasoned Action Approach van Fishbein en Ajzen (2010), maar dat meer distale variabelen, zoals het aantal keren dat men onveilige seks had in het afgelopen jaar, ook significant bijdroegen aan de verklaring van de variatie in intentiescores onder de deelnemers. Kwalitatieve data in hoofdstuk 2 laten vervolgens zien dat jongeren alle drie gedragingen inderdaad relevant achten in het voorkomen van seksueel overdraagbare aandoeningen, maar dat condoomgebruik, alhoewel relatief veel toegepast, vooral wordt gezien als een noodzakelijk kwaad dat het plezier in seks vermindert, en dat de andere twee gedragingen consistent het minst populair zijn onder jongeren. Het advies om risicovolle seksuele praktijken te vermijden werd herhaaldelijk geassocieerd met saaiheid en werd verworpen omdat daarmee het plezier in seks zou worden gereduceerd. Het vragen naar het seksuele verleden van de partner werd gezien als iets waarvoor men zich schaamt en werd ook als onplezierig ervaren. Vooral jongeren die een lage intentie hadden tot het uitvoeren van alle drie gedragingen wezen het vragen naar het seksuele verleden van een partner af. Opmerkelijk daarentegen was de bevinding in hoofdstuk 4 dat het vragen naar het seksuele verleden van een nieuwe partner niet werd afgewezen als deelnemers in een toestand van seksuele opwinding verkeerden, blijkend uit positievere scores op metingen van de impliciete en expliciete attitude ten aanzien van dit gedrag dan wanneer zij niet seksueel opgewonden waren. Deze bevindingen suggereren dat seksuele opwinding niet per se een bedreiging is voor het vertonen van seksueel veilig gedrag, maar juist de remming kan wegnemen bij gedrag dat normaal als beschamend wordt gezien. Bevindingen in hoofdstuk 5 laten bovendien zien dat schaamte de vertaling van de intentie tot condoomgebruik naar gedrag in de weg kan staan. Door gebruik te maken van elektro-encefalografie (EEG) werd steun gevonden voor de hypothese dat een hoge intentie alleen onvoldoende is voor het bewerkstelligen van condoomgebruik als de implementatie daarvan wordt geassocieerd met schaamte.

Om te toetsen of een combinatie van gamified gedragsveranderingsmethodieken, namelijk evaluatieve conditionering, selectieve response inhibitie training en training van het werkgeheugen, de intentie tot het uitvoeren van de drie beschermende gedragingen kan verhogen, werd een serious game voor jongeren in de leeftijd van 18-24 jaar ontwikkeld. In hoofdstuk 3 werd aan de hand van focus group interviews onderzocht welke 
voorkeuren jongeren hebben als het gaat om de inhoud en vormgeving van een dergelijk serious game. Deelnemers hadden de voorkeur voor een levensecht ontwerp met avatars die naar eigen gelieven kunnen worden vormgegeven. Tevens zouden ze graag informatiecomponenten toegevoegd zien worden om verschillende seksueel veilige gedragingen verder toe te lichten waaronder het gebruik van verschillende manieren om direct contact met infectiebronnen te vermijden. Bovendien stelden deelnemers voor een avatar toe te voegen die onder de invloed is van alcohol, om jongeren bewust te maken van het verlies van controle dat optreedt als iemand onder invloed is.

De uiteindelijke game heeft tot doel de impliciete en expliciete determinanten van de drie gedragingen tot het hebben van veilige seks te versterken. De resultaten verkregen uit een experimentele toets van de game staan beschreven in hoofdstuk 6. De resultaten laten zien dat alleen wanneer alle drie veranderingsmethodieken actief waren tijdens het spelen van het spel sommige expliciete determinanten werden versterkt, maar dat tegelijkertijd andere determinanten, in het bijzonder de intenties, werden verzwakt na het spelen van drie of vijf sessies van het spel. Geconcludeerd werd dat het spelen van vijf sessies niet voldoende is voor het bewerkstelligen van een robuuste attitudeverandering en dat het tegelijkertijd willen veranderen van de drie gedragingen mogelijk te veel van het goede is. Het lijkt erop dat ook in een virtuele omgeving er sprake zou kunnen zijn van less is more. 
Valorization 

The term knowledge valorization was defined by the National Valorization Committee in 2011 as the "process of creating value from knowledge, by making knowledge suitable and/or available for social (and/or economic) use and by making knowledge suitable for translation into competitive products, services, processes and new commercial activities". The conjunction "and" - linking the first part of this definition related to the creation of value through knowledge with the second part of this definition related to the valorization of knowledge mainly in light of its economic impact - suggests that the term value is defined more as the material or monetary worth of knowledge, and less as the usefulness of knowledge in the process of scientific progress. This is a dangerous road as it may lead to understanding knowledge as a marketable product representing a specific worth that can be compared to the price paid for it by funding entities, thereby reducing knowledge to an economic asset with a required return on investment. Rather than asking about the economic gain of knowledge resulting from a study or a research line, a more appropriate question to ask may be about what we do not know and where we can make progress. It was this curiosity-driven research that lead to unexpected revolutionary discoveries. For example, radio waves were not called "radio waves" when they were mathematically predicted by James Clerk Maxwell in 1867, because radios did not exist yet. Only after Heinrich Hertz demonstrated the reality of these waves in 1887 (and coined the term "Hertzian waves") and Guglielmo Marconi used them for communication by developing a transmitter and receiver system, these waves revolutionized communication and were renamed "radio waves" around 1912. However, Maxwell's intent was not to discover how to revolutionize communication. His field of research was fundamental mathematical physics where he formulated the now well-known Maxwell's equations for electromagnetism.

The view of knowledge as a product can, of course, be appropriate for specific branches of science (e.g., medical science), but it can hardly be applied to humanities or social sciences. Especially, research in the latter branch generates knowledge that represents more than economic value alone. For instance, research in the field of psychology, focusing on improving health, well-being, and pro-social attitudes on a personal and group level, contributes to improving the social fabric people live in. If the freedom of curiosity-driven research and/or research in domains that do not promise direct economic gains is reduced (or outright eliminated), science risks to sacrifice knowledge that has its own intrinsic value for a short-sighted race to win economic competition by generating knowledge that international markets demand to produce items that promise economic gains. As the focus of this dissertation is to improve safer-sex behavior of young people and thereby reducing the number of young people who acquire preventable sexually transmitted infections (STIS) - including infections with the human immunodeficiency virus (HIV) leading to the acquired immune deficiency syndrome (AIDS) - the honored reader should decide for her or himself how many infections constitute a (not only public health) problem worth attention. As the practical relevance and the innovative nature of the serious game that emerged out of this line of work has 
been stated in detail throughout this dissertation, they will be repeated here only briefly. Reducing the numbers of preventable STIs in a young population does not seem to be a task that has to be defended, rather than a task that needs further (also financial) support.

\section{Relevance}

Given that young people at risk of acquiring preventable STIs are rarely prioritized in STI prevention programs, testing a novel and innovative way to deliver behavior-change techniques aimed at reducing the STI rate of these young people is a valuable step toward including this population - that spends an increased amount of time in virtual online environments - in STI prevention programs. With a quarter of a billion young people aged 15-24 years acquiring a curable STI for the first time - accounting for nearly half of all new curable STIs - it is of particular importance to identify new ways to successfully address this population, as sexually transmitted diseases (STDs) also facilitate HIV transmission.

\section{Target groups}

The serious game developed during this project aimed at increasing the safer-sex behavior of young people, aged between 18 and 25 years. Thus, young people of this age group are the main targets of the intervention, delivered as a serious game. However, depending on local legal requirements, the age range could be adapted to adolescents as well. Further, this dissertation must be of interest to developers of serious games aiming at reducing STI rates among young people (e.g., public health officials), as it contains new information about, for example, preferences young people have toward presenting the topic of safe sexual intercourse in an online gaming environment. Additionally, this dissertation targets researchers who are interested in finding novel ways to present sensitive topics to a young population by using newly emerging virtual communication channels.

\section{Activities and products}

This work is also of interest to anyone, especially funding entities, who wishes to support research to improve serious games as a delivery tool for interventions. Given the rising costs of developing serious games (e.g., including virtual reality scenarios), funding the development of interventions using a gamification approach must be adapted not only to the costs of research but foremost to the reality of high development and production 
costs for a product that risks being outdated within a short period of time. As an economically driven return on investment can hardly be expected, there is, however, the possibility of a social/societal return on investment, by significantly reducing STI rates of young people that can be reached easier by serious online games than by more conservative means of distribution.

\section{Innovation}

Combining different behavior-change techniques in a gamified online environment that targets three protective behaviors in young people has never been done before. This innovative and novel approach allowed us to better understand what young people need and expect in a virtual online environment that focuses on safe sexual intercourse. It further showed that combining various techniques and focusing on several outcome behaviors in one game is a complex task that needs more research to understand if and under what circumstances such combinations could be effective.

\section{Schedule and implementation}

Our research has shown that a limited number of gaming sessions aiming at implementing three outcome behaviors (or at least the intention to perform them) is not sufficient and that simply increasing the number of sessions can be counterproductive. By having done the first step toward combining several behavior-change techniques aimed at three outcome behaviors in a serious gaming environment, future research in how to develop an attractive and holistic game could be stimulated. Further, a website that accompanied the project informed a broader audience - by using blog posts - about risks of unsafe sexual intercourse and benefits of safer-sex behavior, thereby contributing to the valorization of knowledge. 



\section{About the author}

Phil Brüll was born on February $8^{\text {th }}, 1970$ in Eupen, Belgium. After graduating from secondary school in 1989, he was admitted to the Medical School at the Université de Liège in Belgium. He later continued his pre-clinical education at the RheinischWestfälische Technische Hochschule in Aachen, Germany, but discontinued his medical studies after the pre-clinical education. During this time, he was trained as an emergency paramedic at the Ecole Provinciale d'Aide Médicale Urgente in Liège, Belgium and worked as an emergency paramedic on ambulance and the emergency physician intervention team at the Fire Brigade in Eupen, Belgium. Phil was also a trainer for new paramedics and paramedics following annually certification training at the Ecole Provinciale d'Aide Médicale Urgente in Liège until 2006. He then decided to learn more about individual decision-making processes and started his undergraduate studies at the University College at Maastricht University, the Netherlands. Phil obtained his Bachelor of Liberal Arts and Sciences degree in 2010. Subsequently, he joined the Cognitive Neuroscience track of the Psychology Master at Maastricht University, from which he graduated in 2011. For his Master thesis, he conducted research examining electrophysiological effects for affirmative and negative goal framing strategies. From 2011 to 2018 he was a doctoral candidate at the Department of Work and Social Psychology of the Faculty of Psychology and Neuroscience at Maastricht University. His research was about gamification of specific behavior-change techniques aiming at improving young people's safer-sex behavior. Since 2017, Phil also works at the Student Services Centre of Maastricht University, where he is currently developing a behaviorchange intervention, targeting alcohol and drug abuse among students. 